\title{
Clasificación topológica del flujo hamiltoniano de algunos problemas roto-traslatorios
}

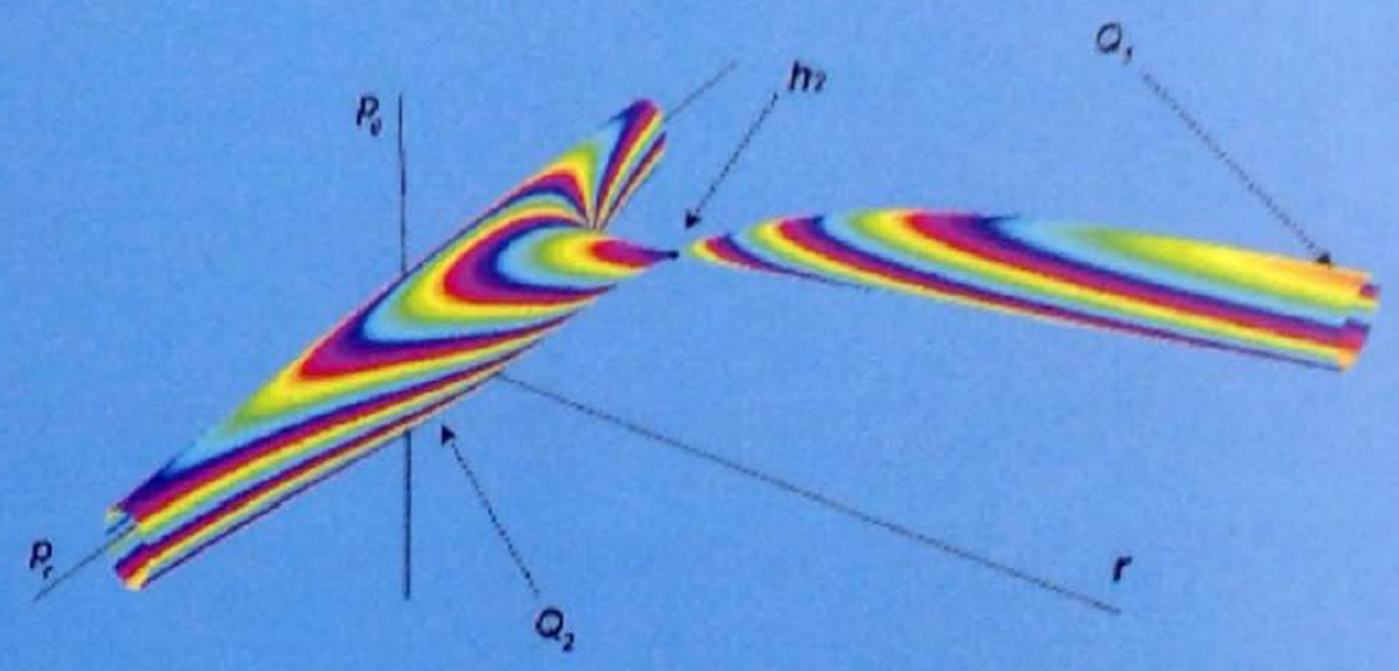

Elena Soledad Jiménez Ayala

UNIVERSIDAD POLITÉCNICA DE CARTAGENA DEPARTAMENTO DE MATEMÁTICA APLICADA Y ESTADÍSTICA 


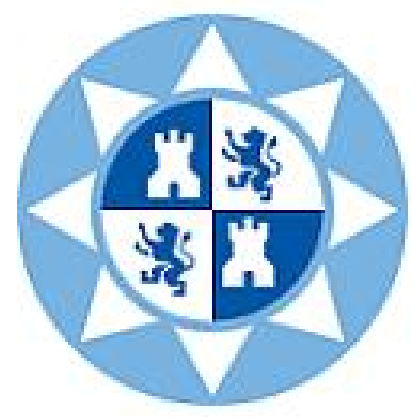

UNIVERSIDAD POLITÉCNICA DE CARTAGENA DEPARTAMENTO DE MATEMÁTICA APLICADA Y ESTADÍSTICA

Tesis doctoral:

\section{Clasificación topológica del flujo hamiltoniano de algunos problemas roto-traslatorios}

Elena Soledad Jiménez Ayala 


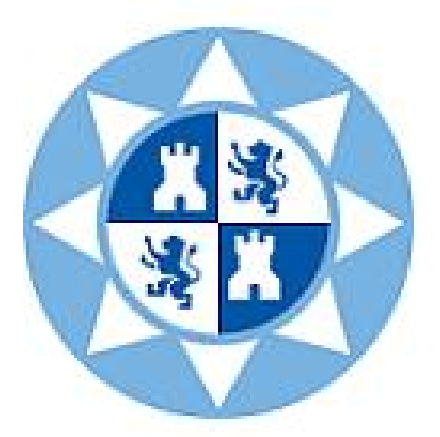

UNIVERSIDAD POLITÉCNICA DE CARTAGENA DEPARTAMENTO DE MATEMÁTICA APLICADA Y ESTADÍSTICA

Tesis doctoral:

Clasificación topológica del flujo hamiltoniano de algunos problemas roto-traslatorios

Elena Soledad Jiménez Ayala

Director:
Juan Antonio Vera López
Codirector:
Antonio Vigueras Campuzano




\section{Universidad}

Politécnica

de Cartagena

DT-16

\section{CONFORMIDAD DE SOLICITUD DEAUTORIZACIÓN DE DEPÓSITO DE TESIS DOCTORAL POR EL/LA DIRECTOR/A DE LA TESIS}

D./Da. Juan Antonio Vera López Director/a de la Tesis doctoral "Clasificación topológica del flujo hamoltoniano de algunos problemas roto-traslatorios" y D. Antonio Vigueras Campuzano, codirector y tutor de la tesis

\section{INFORMAN:}

Que la referida Tesis Doctoral, ha sido realizada por $D / D^{a}$. Elena Soledad Jiménez Ayala dentro del programa de doctorado Tecnologías Industriales, dando mi conformidad para que sea presentada ante la Comisión de Doctorado para ser autorizado su depósito.

La rama de conocimiento en la que esta tesis ha sido desarrollada es:

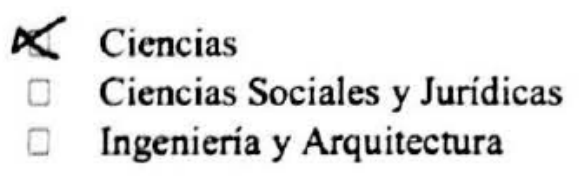

En Cartagena, a 21 de Junio de 2016

EL/LA DIRECTOR/A DE LA TESIS

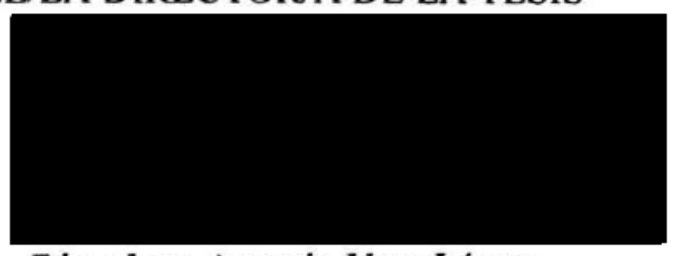

Fdo.: Juan Antonio Vera López

EL CODIRECTOR DE LA TESIS

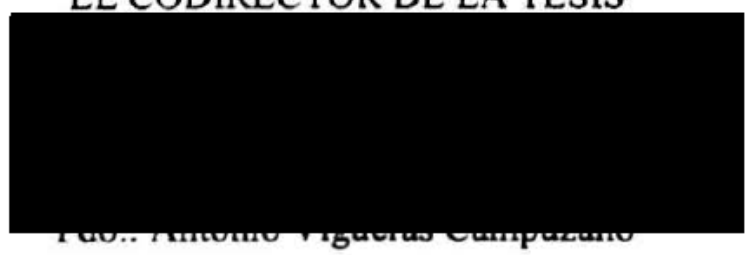




\section{Universidad}

Politécnica

de Cartagena

\section{CONFORMIDAD DE DEPÓSITO DE TESIS DOCTORAL POR LA COMISIÓN ACADÉMICA DEL PROGRAMA}

D/D". Juan Suardíaz Muro. Presidente/a de la Comisión Académica del Programa de Doctorado "Tecnologías Industriales".

\section{INFORMA:}

Que la Tesis Doctoral titulada, "Clasificación topológica del flujo hamiltoniano de algunos problemas roto-traslatorios", ha sido realizada, dentro del mencionado programa de doctorado, por D'. Elena Soledad Jiménez Ayala, bajo la dirección y supervisión de los Dres. D. Juan Antonio Vera López y D. Antonio Vigueras Campuzano.

En reunión de la Comisión Académica de fecha 1610012916 , visto que en la misma se acreditan los indicios de calidad correspondientes y la autorización del Director de la misma, se acordó dar la conformidad, con la finalidad de que sea autorizado su depósito por la Comisión de Doctorado.

La Rama de conocimiento por la que esta tesis ha sido desarrollada es:

đCiencias

$\square$ Ciencias Sociales y Jurídicas

$\square$ Ingeniería y Arquitectura

En Cartagena, a 21 de junio de 2016

EL PRESIDENTE DE LA

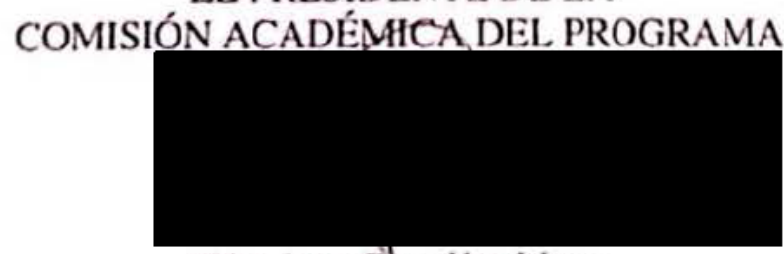

Fdo: Juan Shardíaz Muro 
Porque al final todo llega... 


\section{Agradecimientos}

Este proyecto está dedicado a las personas más importantes de mi vida. Aquellas que me ha hecho ser lo que soy ahora, que me quieren y me apoyan y sobre todo, me valoran. Es por ello que quiero, a todos ellos, dedicar unas palabras.

Ángel y Marisol, mis padres. Sin su ayuda, sin su apoyo, sin su cariño, sin su esfuerzo, jamás hubiera podido hacerlo. Muchos son los sacrificios que han hecho para darme una educación y una formación, no solo académica, sino también personal. Por esa paciencia que han tenido y por esas cenas tardías que me han permitido alimentarme en esos días que he llegado tarde a casa y sin ganas de cocinar, quiero darles las gracias.

Mis hermanos, que aunque a veces no hablemos o no nos veamos tanto como antes, sois un pilar importantísimo en mi vida.

A Antonio, por su gran paciencia y por su ayuda. Gracias por los fines de semana recogiéndonos temprano porque al día siguiente tenía que trabajar y por coger el coche (tú ya me entiendes). Sé que tú también te has sacrificado, pero has hecho que todo sea mucho más fácil. Pero por encima de todo, gracias por tu cariño.

A Javi, quien ha tenido que aguantar mi mal humor cuando he estado agobiada y siempre se ha ofrecido a ayudarme durante todos estos años.

Antonio Vigueras Campuzano, que me ha ayudado muchísimo. Fue él quien que me introdujo en el tema de la Mecánica Celeste. Gracias por tus consejos y aportaciones.

Juan Antonio Vera López, que fue quien tomó el mando de esta tesis y me entregó ese paper de Llibre que cambió mi vida. Gracias por tu ayuda y por enseñarme tanto.

Y en especial, a María del Carmen Balsas Ramón, ya que sin ella este proyecto jamás hubiera sido posible. Muchas son las horas que hemos pasado juntas en el despacho y muchos los altibajos que hemos compartido. Esas horas a las que salíamos casi de madrugada y esas pizzas a medianoche no se me olvidarán jamás. Gracias por estar siempre ahí. 


\section{Índice general}

1. Introducción 1

1.1. Introducción histórica y planteamiento del problema . . . . . . . . . . . . 1

1.2. Objetivos y metodología . . . . . . . . . . . . . . . 3

1.3. Resumen y conclusiones . . . . . . . . . . . . . . . . . 4

2. Fundamentos mecánico-geométricos de la dinámica clásica 7

2.1. Formulaciones lagrangiana y hamiltoniana . . . . . . . . . . . . . 7

2.1.1. Sistemas dinámicos . . . . . . . . . . . . . . . 7

2.2. Ecuaciones de Lagrange y de Hamilton . . . . . . . . . . . . . . . . . . . . 8

2.3. Transformaciones canónicas y funciones generatrices . . . . . . . . . . . . 9

2.4. Ecuación de Hamilton-Jacobi . . . . . . . . . . . . . . . . . . . . . . 11

2.5. Teoremas de Liouville y Liouville-Arnold . . . . . . . . . . . . . . . . . 12

2.6. Elementos de mecánica-geométrica . . . . . . . . . . . . . . 15

2.6.1. Introducción y terminología . . . . . . . . . . . . . . . 15

2.6.1.1. Notaciones usuales . . . . . . . . . . . . . . . 16

2.6.2. Grupos y álgebras de Lie . . . . . . . . . . . . . . . . 16

2.6.2.1. Situándonos dentro de nuestro problema . . . . . . . . . . 16

2.6.2.2. Definiciones y primeras propiedades . . . . . . . . . . . 17

2.6.3. Acciones de grupos de Lie . . . . . . . . . . . . . . . . . . . 20

2.7. Variedades simplécticas y de Poisson . . . . . . . . . . . . . . . . 21

2.8. Aplicaciones momento y teoremas de reducción . . . . . . . . . . . . . 24

2.8.1. Aplicaciones momento . . . . . . . . . . . . . . . . . 24

2.8.2. Teoremas de reducción . . . . . . . . . . . . . . . 26

3. Dinámica hamiltoniana de un giróstato en el problema de $n$ cuerpos 29

3.1. Introducción . . . . . . . . . . . . . . . . . . . 29

3.2. Espacios de configuración y fásico . . . . . . . . . . . . . . . 32

3.3. Simetrías y reducción . . . . . . . . . . . . . . . . . 33

3.3.1. Reducción por el grupo de traslaciones . . . . . . . . . . . . . . . 34

3.3.2. Reducción por medio del grupo de rotaciones $\mathbf{S O}(3) \ldots . . . .36$

3.4. Expresión de la energía potencial . . . . . . . . . . . . . . . 37

3.5. Ecuaciones del movimiento . . . . . . . . . . . . . . . . . . . . . . 39

3.5.1. Funciones de Casimir. Integrales del momento angular . . . . . . . 39

3.5.2. Equilibrios relativos . . . . . . . . . . . . . . . . 39

3.5.2.1. Carácter variacional del los equilibrios relativos . . . . . . 40

3.6. Variedades invariantes . . . . . . . . . . . . . . . . . . . 41

3.6.1. Movimiento plano . . . . . . . . . . . . . . . . . . . 41 
4. Dinámica hamiltoniana de giróstato en interacción newtoniana con sólido rígido esférico

4.1. Introducción . . . . . . . . . . . . . . . . . . . . . . . 43

4.2. Ecuaciones del movimiento . . . . . . . . . . . . . . . . . . 43

4.3. Funciones de Casimir. Integrales del momento angular . . . . . . . . . . . . 45

4.4. Dinámica hamiltoniana aproximada . . . . . . . . . . . . . . 45

4.4.1. Variedades invariantes del movimiento . . . . . . . . . . . 46

4.5. Potencial ampliado. Regiones de Hill . . . . . . . . . . . . . . . . 47

4.5.1. Introducción . . . . . . . . . . . . . . . . . . . . . . 47

4.5.2. Obtención del potencial ampliado empleando el lagrangiano . . . . 47

4.5.3. Obtención del potencial ampliado algebraicamente . . . . . . . . . 48

4.5.4. Variedades de energía y momento constantes. Regiones de Hill . . . 49

4.6. Estudio cualitativo del flujo hamiltoniano asociado a $\mathcal{H}$. . . . . . . . . . 49

4.6.1. Introducción . . . . . . . . . . . . . . . . . . . . . 49

4.6.2. Topología de las regiones de Hill . . . . . . . . . . . . . . . 50

4.6.3. Estudio cualitativo del flujo hamiltoniano . . . . . . . . . . . 53

4.6.4. Puntos de equilibrio de $\mathcal{H} \ldots \ldots . \ldots . . \ldots 53$

4.6.5. Extremos relativos de la superficie de energía $g^{-1}(h) \ldots \ldots . . . \quad 54$

4.6.6. Clasificación topológica de $E_{h}$ e $I_{h k} \ldots \ldots$. . . . . . . . . 57

4.7. Variables acción-ángulo . . . . . . . . . . . . . . . . . 62

4.8. Variables de Delaunay . . . . . . . . . . . . . . . . . . . . . 65

4.9. Clasificación de órbitas . . . . . . . . . . . . . . . . . . 66

4.10. Elemento keplerianos modificados . . . . . . . . . . . . . . . 68

4.11. Conclusiones . . . . . . . . . . . . . . . . . . . . . . 69

$\begin{array}{ll}\text { 5. Un sistema Manev en referencia rotante } & \mathbf{7 1}\end{array}$

5.1. Introducción . . . . . . . . . . . . . . . . . . . 71

5.2. Potencial ampliado. Regiones de Hill . . . . . . . . . . . . . . . . . 72

5.3. Topología de las regiones de Hill . . . . . . . . . . . . . . . . . . 73

5.4. Estudio cualitativo del flujo hamiltoniano . . . . . . . . . . . . . . 76

5.5. Variables acción-ángulo . . . . . . . . . . . . . . . 86

5.6. Conclusiones . . . . . . . . . . . . . . . . . . . 87

6. Una aproximación al Main Problem del satélite artificial $\quad 89$

6.1. Introducción . . . . . . . . . . . . . . . . . . . 89

6.2. Potencial ampliado. Regiones de Hill . . . . . . . . . . . . . . . . . . . 92

6.3. Topología de la regiones de Hill . . . . . . . . . . . . . . . . . . . . . . . . . . . . . . 93

6.4. Estudio cualitativo del flujo hamiltoniano . . . . . . . . . . . . . 96

6.4.1. Trayectorias acotadas . . . . . . . . . . . . . . . 97

6.4.2. Clasificación topológica de $E_{h}$ e $I_{h k} \ldots \ldots$. . . . . . . . . . . . 99

6.5. Conclusiones . . . . . . . . . . . . . . . . . . . . 105

$\begin{array}{ll}\text { A. Cálculo de los equilibrios de } \mathcal{H} & 109\end{array}$

$\begin{array}{ll}\text { B. Extremos relativos de la superficie } & 113\end{array}$

$\begin{array}{ll}\text { C. Topología de } I_{h k} & 123\end{array}$

D. Estudio numérico de las órbitas 139 
Lista de figuras

Lista de tablas

151

Bibliografía 153 


\section{Abstract}

This thesis is made up of six main chapters and some different appendices. Let us see briefly their most important results:

In the chapter called introduction, the metodology which is going to be used is described, as well as the principal objectives we want to achieve in this thesis.

In chapter 2 we introduce some important concepts which are needed to understand this thesis. Thus, the Classical Mechanics is described; besides, it comes from the restricted Relativity Theory. First of all, the Lagrangian and Hamiltonian notation is introduced and so the Dynamical systems. Secondly, the Langrange and Hamiltonian equations are given. Thirdly, the canonical transformations and their characteristical functions are studied too. Later, the Hamilton-Jacobi equations are given and also the Liouville and Liouville-Arnold theorems. Then the Lie groups, the Lie algebras and the Lie actions are presented. As they are quite important for us, the simplectic and Poisson manifolds are studied too, as well as the momentum maps and some reduction theorems, which will allow us to reduce significantly the problems we will study during this work.

Through chapter 3, the non-canonical Hamiltonian dynamics of a gyrostat in Newtonian interaction with $\mathrm{n}$ spherical rigid bodies is considered. By using the symmetries of the system we obtain two reductions. Then, working in the reduced problem, we calculate the expression for the potential and the equations of motion, a Casimir function of the system and the equations that determine the relative equilibria. Some global conditions for the existence of relative equilibria are given. Furthermore, we give the variational characterization of these equilibria and three invariant manifolds of the problem; being calculated the equations of motion in these manifolds, which are described by means of a canonical Hamiltonian system. Lastly, the equations of motion for a planar motion are described in one of these invariant manifolds.

In chapter 4 we describe the Hamiltonian dynamics, in some invariant manifolds of the motion of a gyrostat in Newtonian interaction with a spherical rigid body. Considering a first integrable approximation of this roto-translatory problem, by means of LiouvilleArnold theorem and some specifics techniques, we obtained a complete topological classification of the phase flow associated to this system. The action-angle variables regions are obtained and the Delauny variables too. Thanks to the action-angle variables, we are able to calculate the modified Keplerian elements of this problem, useful to elaborate a perturbation theory. And last but not least, for one specific case the orbits are classified. We must take into account that the results of this work have a direct application to the study of two body roto-translatory problems, where the rotation of one of them influences strongly in the orbital motion of the system. In particular, we can apply these results to binary asteroids. 
In chapter 5 we have considered the Manev systems in a rotating reference frame. We describe the Hamiltonian dynamics, in the invariant manifolds $E_{h}, J_{k}$ and $I_{h k}$ by means of Liouville-Arnold theorem and some specific techniques. We also obtain a complete topological classification of the phase flow associated to this system. Finally, the actionangle variables are obtained. These variables allow us to calculate the modified Keplerian elements of this problem useful to elaborate a perturbation theory.

In chapter 6 we consider the Kepler problem with a perturbation; this is an approximation to the Main Problem of the artificial satellite. We make an analytical, numerical and topological study of Hamiltonian dynamics for a simplified case where we only considered the first one and second dominant term of the gravitational potential. Using the Liouville-Arnold theorem and a particular analysis of the momentum map in its critical points we obtain a complete topological classification of the different invariant sets of the phase flow associated to this problem.

Finally, during the appendices we can see the algorithms and calculations which have been done to obtain the critical points and the relative equilibria, to study the topology of $I_{h k}$ and to classify the orbits of one of the main cases. 


\section{1}

\section{Introducción}

\subsection{Introducción histórica y planteamiento del pro- blema}

Uno de los problemas más estudiados de la Mecánica Celeste en los últimos cincuenta años es el de movimiento de un satélite artificial terrestre. Es bien sabido que, considerado como un sólido rígido, su movimiento se descompone en dos, el movimiento orbital de su centro de masas y el de rotación del satélite en torno a dicho punto. En general, dichos movimientos están relacionados, aún en el supuesto de despreciar las perturbaciones no gravitatorias; aunque en la práctica, debido al pequeño tamaño del satélite en comparación con las dimensiones de la órbita, se considera nula la influencia del movimiento rotatorio sobre el orbital, por lo cual en el estudio de su rotación alrededor del centro de masas se suele suponer conocido el movimiento de este punto. La mayoría de los trabajos publicados en relación con el problema del satélite están en alguna de las dos líneas de estudio anteriormente citadas (orbital o rotacional), abarcando distintos casos particulares de satélites rígidos y diferentes métodos de integración, ya sea por las variables utilizadas, la forma hamiltoniana o no de las ecuaciones del movimiento, el método de perturbaciones usado, el movimiento adoptado para el centro de masas, etc.

En el caso de satélites, Lagrange (1782) encuentra una solución particular al problema del movimiento de un satélite, cuyo centro de masas describe una circunferencia y para el cual los ejes principales de inercia se hallan orientados según el radio vector, la tangente y la binormal a esta órbita circular; solución que corresponde aproximadamente al movimiento de la Luna. En rigor, para darse una idea completa del problema hasta los comienzos del siglo XX, basta seguir la obra de Laplace (1799 a 1825) y la brillante exposición de Tisserand (1891) en su segundo tomo. Trabajos posteriores importantes son los de Woolard (1953) y Kinoshita (1977), Kinoshita y Souchay (1990) pues ejercen decisiva influencia en la confección actual de las efemérides y conducirán, en los próximos años a un refinamiento de las fórmulas de cálculo. Asimismo, en relación a la rotación terrestre cabe destacar los trabajos de Ferrándiz, Getino, Navarro y Escapa (1995, 1997, 2000, 2001, 2002) que estudian la rotación de una tierra elástica.

Señalemos, para completar esta reseña histórico-bibliográfica, que, en el planteamiento general sobre el movimiento conjunto de n sólidos, destacan los trabajos de Duboshin (1958, 1963, 1968, 1972), quien probó la existencia de las diez integrales clásicas. También han aparecido algunos trabajos dedicados a problemas particulares del movimiento de dos o tres sólidos, debidos, entre otros, a Duboshin (1974), Kondurar (1964), E.Cid (1982), 
R.Cid y M.E. San Saturio (1981), A. Elipe y S. Ferrer (1985), etc.

Kinoshita (1972) efectúa la integración del problema de movimiento de dos sólidos en interacción newtoniana (uno esférico y otro triaxial), por el método de Hori, considerando el potencial como una perturbación de la energía cinética. Este mismo problema, para dos sólidos simétricos, ha sido abordado por Ferrándiz (1979), tomando como orden cero la energía orbital, como orden uno la energía cinética de rotación de ambos sólidos y como orden dos el término principal del potencial no kepleriano, integrando el problema según el método de perturbaciones de Deprit.

Ahora bien, el modelo de sólido rígido para representar a los cuerpos celestes cuando estudiamos su movimiento implica la ausencia de movimientos internos o relativos, hipótesis que en algunos casos no es muy adecuada, como probó Volterra (1899) en su estudio de la variación de la latitud sobre la superficie terrestre, explicando las anomalías de la rotación libre terrestre por medio de movimientos internos que no modifican la distribución de masas.

Siguiendo las investigaciones de Volterra, Rumiantsev $(1961,62)$ definió un giróstato como un sistema mecánico $S$, compuesto de un sólido rígido $S^{\prime}$ (también llamado plataforma) y otros cuerpos $S^{\prime \prime}$ conectados al primero, estos otros cuerpos son a su vez variables o rígidos, cuyos movimientos relativos a $S^{\prime}$ no modifican la distribución de masas del sistema $S$.

Como queda indicado, en los trabajos antes mencionados, se considera el movimiento roto-traslatorio de uno o varios sólidos rígidos, en tanto que nuestra memoria estará dedicada al estudio del movimiento de sólidos rígidos o giróstatos indistintamente.

Debemos señalar que, en el problema de movimiento rotatorio de un giróstato alrededor de su centro de masas o cuando dicho punto describe una órbita circular, han sido encontradas algunas soluciones de equilibrio y estudiada su estabilidad en el sentido de Lyapunov, en distintos casos particulares. Los trabajos más relevantes se deben a Rumiansetv (1963, 64,79, 80), Keis (1964), Anchev(1962, 67), Kovalev (1971, 81), Krementulo (1970), M.R.M. Crespo da Silva (1970), R. Longman y otros (1981), Vigueras (1983), M. Pascal (1985), M. Pascal y S. Ia. Stepanov (1991).

En la dinámica hamiltoniana de un giróstato en el problema restringido plano de tres cuerpos, podemos citar, entre otros, los trabajos de investigación de C. Hall (1994, 95, 96, 2008), Elipe, Lanchares (1994, 97, 2001, 2008), Cavas \& Vigueras (1994), Molina \& Mondéjar (2004), Mondéjar, Vigueras \& Ferrer (1999), Wang et al. (1995), Vera \& Vigueras $(2004,2005,2006)$, Balsas et al. (2008, 2008, 2009). En este estudio será necesario, en general, utilizar métodos analíticos, cualitativos y numéricos. En relación con el estudio cualitativo del flujo hamiltoniano que realizaremos en diversos problemas nos será de gran utilidad el trabajo de Llibre et al (2001).

En los últimos tiempos, numerosos trabajos muestran un gran interés sobre las configuraciones de equilibrio relativo y nuevos métodos han sido propuestos por autores como Cushmann y Bates, Marsden, Marsden y Ratiu. Al respecto, sobre el estudio de configuraciones de equilibrio, así com la estabilidad de los mismos podemos citar los trabajos de Wang, Krishnaprasad y Maddocks, Maciejewski, Lian y Chen, Mondéjar, Vera y Vigueras.

En referencia al problema de tres sólidos nos gustaría mencionar que los trabajos de Vidiakin y Duboshine probaron la existencia de equilibrios tipo Euler y Lagrange cuando los cuerpos poseen simetrías, Zhuralev y Petruskii hicieron una revisión de los resultados hasta el año 1990.

Por otra parte, Fanny and Badaoui estudiaron los equilibrios relativos en términos de variables globales en el problema no reducido. En Mondéjar, Vigueras y Ferrer, se 
considera un problema general de tres cuerpos, donde uno de ellos es un giróstato, trabajando en el problema reducido, se proporcionan condiciones necesarias y suficientes sobre la existencia de ciertos equilibrios relativos del problema. Además, se detalla un estudio completo de los equilibrios relativos en la dinámica aproximada de orden cero.

\subsection{Objetivos y metodología}

Consideraremos en nuestro trabajo el movimiento en el problema general de $n$ cuerpos, y en particular, estudiamos el caso del problema del satélite girostático en interacción newtoniana con un sólido rígido esférico, poniendo de manifiesto la influencia de los movimientos internos que no modifican la distribución de masas sobre el movimiento de rotación de la parte rígida del satélite. En el estudio cualitativo, se utilizaremos la formulación hamiltoniana, las variedades invariantes del movimiento y los teoremas de Liouville-Arnold.

Concretando, los objetivos a alcanzar y pasos a seguir serán los siguientes:

1. En primer lugar, se realizará un estudio bibliográfico del problema de movimiento rototraslatorio de un satélite en atracción newtoniana con uno o varios cuerpos.

2. Se recordarán brevemente los resultados geométricos y topológicos básicos relativos a la dinámica del sólido rígido o giróstato.

3. Se planteará el problema general del movimiento de un giróstato en atracción newtoniana con $n$ sólidos rígidos esféricos o puntos materiales, por medio de una función hamiltoniana no canónica. Se considera el caso particular en el que $n$ es igual a dos o tres.

4. Se describirá su dinámica hamiltoniana y se estudiará el movimiento en las fibras de momento angular constante de una determinada variedad invariante del movimiento.

5. Para el problema anterior, utilizando el teorema de Liouville-Arnold se investigará una clasificación topológica de los conjuntos invariantes del flujo fase en un caso particular de este problema.

6. Investigaremos la topología de las regiones de Hill de diversos problemas próximos al anteriormente considerado, entre ellos el caso de un un giróstato en un campo central newtoniano o un problema de dos cuerpos con potencial tipo Manev.

7. Obtención de las variables acción-ángulo para algunos de los problemas considerados.

8. Construcción y utilización del software apropiado para el estudio de la dinámica hamiltoniana de los problemas anteriormente reseñados. 


\subsection{Resumen y conclusiones}

Los principales resultados obtenidos y expuestos en (Balsas, Jiménez y Vera (2007) [7], (2008) [8]; Balsas, Jiménez, Vera y Vigueras (2009) [9], (2016) [10]; Balsas, Jiménez, Guirao y Vera (2009) [12]) así como en esta memoria versan sobre el estudio topológico de la dinámica de diferentes sistemas hamiltonianos derivados de problemas de carácter roto-traslatorio.

Durante este último siglo varios matemáticos han utilizado el estudio topológico para la comprensión de la dinámica de sistemas hamiltonianos, integrables o no. Poincaré fue el mentor intelectual de esta idea, que resultó ser una especie de nuevo enfoque cualitativo para estudiar el flujo de un sistema hamiltoniano. Este método de carácter topológico fue utilizado por Birkhoff (1927) [14], en el estudio del problema de tres cuerpos y de algunos sistemas dinámicos generales, y por Kaplan en el problema de dos cuerpos. Sin embargo, son de gran interés los resultados obtenidos tras la publicación de los artículos "Topología y Mecánica I y II" de Smale ( (1970) [54], [55]). Estas obras de Smale fueron seguidas por muchos autores que extendían los resultados de la topología al problema de $n$ cuerpos, para $n \geq 2$, es decir, el movimiento de $n$ cuerpos que se mueven en el espacio bajo la acción de sus fuerzas gravitacionales mutuas (véase por ejemplo, los documentos de Albouy (1993) [3], Cabral (1999) [16], Casasayas (1984) [18], Fomenko (1988) [28], Llibre (2001) [36].

A continuación haremos un resumen del presente trabajo que consta, con independencia de esta introducción, de cinco capítulos y cuatro apéndices de carácter técnico, que describiremos brevemente.

En el capítulo 2, titulado "Fundamentos mecánico-geométricos de la dinámica clásica", se recuerdan brevemente las ecuaciones de movimiento de un sistema dinámico formado por $N$ cuerpos materiales, sometidos a ligaduras holónomas lisas, que interaccionan según las leyes de Newton, así como las formas langragiana y hamiltoniana de estas ecuaciones, las transformaciones canónicas, las funciones generatrices y la teoría de Hamilton-Jacobi. Se continua recordando el teorema de Liouville sobre integrabilidad por cuadraturas de sistemas hamiltonianos, y el teorema de Louville-Arnold que nos será de gran utilidad en los capítulos siguientes para describir la topología de los subconjuntos invariantes del espacio fásico de un sistema hamiltoniano integrable. Más adelante, presentaremos algunos elementos de la moderna mecánica-geométrica, destacando los grupos y las álgebras de Lie y las acciones de los grupos de Lie sobre una variedad diferenciable $n$ dimensional $\mathbf{M}$, que será en unas ocasiones nuestro espacio de configuración y en otras el espacio fásico de los problemas en consideración. Finalmente, debido a la importancia que tienen a la hora de simplificar algunos de los problemas que consideramos en capítulos posteriores, se estudiarán las variedades simplécticas y de Poisson, así como las aplicaciones momento y algunos teoremas de reducción, para sistemas hamiltonianos con simetría, ya sea sobre variedades de Poisson o simplécticas.

El capítulo 3, titulado "Dinámica hamiltoniana de un giróstato en el problema de $\mathrm{n}$ cuerpos", está dedicado al estudio de la dinámica hamiltoniana no canónica del problema de $n+1$ cuerpos en atracción newtoniana, donde $n$ de ellos son sólidos rígidos con distribución esférica de masas (o masas puntuales) y el otro es un giróstato triaxial cuyo momento girostático es constante. Tras definir los espacios de configuración y fásico relativos al problema, se plantea este en forma hamiltoniana no canónica, luego utilizamos 
la reducción del producto semidirecto, por medio de la acción del grupo de Lie $\mathbf{S E}(3)$, siguiendo la línea de Vera [59] (2004) y Vera y Vigueras (2006) [62], se procederá en dos pasos, generalizando el trabajo de Mondéjar et al. (2001) [48], en un primer paso se procederá mediante una reducción simpléctica debido a la acción del grupo de translaciones $\mathbb{R}^{3}$. En una segunda fase, se procederá a una reducción de Poisson mediante la acción del grupo de Lie $\mathbf{S O}(3)$. Trabajando en el problema reducido calcularemos la expresión del potencial y las ecuaciones del movimiento, así como la función de Casimir asociada al sistema y las ecuaciones que determinarán los equilibrios relativos. También se dan algunas condiciones globales para su existencia, además de la caracterización variacional de los mismos y tres variedades invariantes del problema. Posteriormente, serán obtenidas las ecuaciones del movimiento en estas variedades, las cuales serán descritas por medio del sistema del hamiltoniano canónico. Finalmente, se escriben las ecuaciones del movimiento plano de un giróstato en dichas variedades invariantes.

En el capítulo 4, titulado "Dinámica hamiltoniana de un giróstato en interacción newtoniana con un sólido rígido esférico", se plantea la formulación hamiltoniana no canónica de este problema como caso particular del problema general visto en el capítulo anterior, se obtiene una función de Casimir y su dinámica aproximada por truncación del potencial gravitatorio. Luego se trabaja con una aproximación integrable de un problema de tipo roto-traslatorio del movimiento plano de un giróstato en interacción newtoniana con un sólido rígido esférico o masa puntual. Para ello, haremos el estudio en las fibras de valor constante del momento angular total $\left(L_{M_{C}}=L\right)$ en la variedad invariante $\mathbf{M}_{C}$. Se describirá la dinámica hamiltoniana en dicha variedad de las superficies $E_{h}$ e $I_{h k}$, por medio del teorema de Liouville-Arnold y otras técnicas específicas. También se obtendrá una clasificación topológica completa para el flujo fásico asociado al sistema. Las variables acción-ángulo y la región del espacio fásico serán obtenidas, así como nuestro hamiltoniano expresado en dichas variables. De la misma manera, se procederá a calcular las variables de Delaunay y el hamiltoniano correspondiente, útiles para poder obtener los elementos keplerianos modificados que servirán para la elaboración de una teoría de perturbaciones. Por último, se hará un estudio de las órbitas para un caso particular y se calcularán las ecuaciones planetarias. Los resultados obtenidos, tienen una aplicación directa al estudio de dos cuerpos de tipo roto-traslatorios, en particular al caso de asteroides binarios, donde la rotación de uno de ellos influye fuertemente en el movimiento orbital del sistema, el otro debe ser una masa puntual o un cuerpo esférico y ambos deben estar a una distancia mucho mayor que sus propias dimensiones. Un ejemplo de este caso es la situación de 45 Eugenia y su luna Petit-Prince (Marchis et al (2004) [42]).

En el capítulo 5, titulado "Un sistema Manev en referencia rotante", utilizaremos las mismas técnicas que en los precedentes para estudiar el problema de Manev en un sistema de referencia rotante, que es una generalización del trabajo de Llibre (2001) [36]. A través de diferentes técnicas y del teorema de Liouville-Arnold, podremos realizar el estudio cualitativo del sistema hamiltoniano en cuestión. Gracias al cálculo de los puntos críticos del potencial ampliado, definiremos y calcularemos las regiones de Hill y su topología. Tras definir lo que llamaremos superficie de energía, se hará la clasificación topológica de los subconjuntos invariantes $E_{h}$ e $I_{h k}$. Finalmente, tanto las variables acción-ángulo como el hamiltoniano expresado en dichas variables serán obtenidos. Estas nuevas variables nos permitirán calcular los elementos de Kepler del problema, útiles para elaborar una teoría de perturbaciones. 
En el capítulo 6, titulado "Una aproximación al Main Problem del satélite artificial", se considerará el problema principal del satélite artificial (ASP) en dimensión 2, que como sabemos es integrable. El objetivo de este capítulo será usar las técnicas anteriormente utilizadas para describir el flujo hamiltoniano desde un punto de vista topológico.

Por último, se puede ver en los apéndices, los algoritmos y cálculos que se han creado para poder obtener los puntos críticos y los equilibrios relativos, así como los necesarios para el estudio de la topología de los subconjuntos invariantes $I_{h k}$ y para realizar el estudio numérico de las órbitas.

Las referencias que otros autores han hecho de dos de los artículos que se han extraído de esta tesis pueden consultarse en los siguientes enlaces:

- El artículo "The motion of a gyrostat in a central gravitational field: phase portraits of an integrable case" es citado en estos 14 papers que pueden obtenerse en:

https://scholar.google.es/scholar?bav=on.2,or.\&bvm=bv.124817099,d.d24

$\& \mathrm{biw}=1680 \& \mathrm{bih}=886 \& \mathrm{dpr}=1 \& \mathrm{um}=1 \& \mathrm{ie}=\mathrm{UTF}-8 \& \operatorname{lr} \& \mathrm{cites}=7993730193884344845$

- El artículo "Qualitative analysis of the phase flow of an integrable approximation of a generalized roto-translatory problem" se cita en estos siete papers que pueden que pueden obtenerse en:

https://scholar.google.es/scholar?cites=12364346189433917136\&as_sdt=2005 \&sciodt $=0$ 


\section{2}

\section{Fundamentos mecánico-geométricos de la dinámica clásica}

\subsection{Formulaciones lagrangiana y hamiltoniana}

Para algunos de los pioneros de la Física, el movimiento de cuerpos materiales constituyó una de las primeras investigaciones. Es a partir de sus esfuerzos, que se ha evolucionado a lo que se conoce como Mecánica Analítica, Dinámica o simplemente Mecánica. Posteriormente, se le ha puesto el término de Mecánica clásica para distinguirla de la rama de la Física de las modernas teorías físicas, especialmente de la Mecánica cuántica. Esta Mecánica clásica que aqui vamos a describir proviene de la teoría de la Relatividad restringida. Como todo, ésta se basa en algunos principios fundamentales que de forma breve (véase por ejemplo libros como Goldstein (1988) [31] para ver un desarrollo más exhaustivo) se repasarán a lo largo de este capítulo.

\subsubsection{Sistemas dinámicos}

Supondremos un sistema dinámico formado por $N$ puntos materiales $P_{i}(i=1, \ldots, N)$ con masas $m_{i}$, cuya posición viene expresada en un sistema inercial por los vectores $\mathbf{x}_{i}$.

La dinámica de este sistema de puntos viene descrita por el conjunto de ecuaciones resultante de la aplicación de la ecuación fundamental de Newton a cada una de las partículas, es decir,

$$
\mathbf{F}_{i}=\frac{d \mathbf{p}_{i}}{d t}(i=1, \ldots, N)
$$

donde $\mathbf{p}_{i}=m \dot{\mathbf{x}}_{i}$ es el momento lineal de la partícula $P_{i}$.

Lo normal es que los puntos $\mathbf{P}_{i}$ no se mueven libremente sino que estén sujetos a una serie de condiciones o ligaduras holónomas lisas dadas por

$$
f\left(\mathbf{x}_{1}, \mathbf{x}_{2}, \ldots, \mathbf{x}_{N}, t\right)=0 .
$$

Como solemos referirnos a cada partícula por su vector $\mathbf{x}_{i}$ (de tres coordenadas cartesianas), un sistema de $N$ puntos viene representado por $3 N$ coordenadas. Si el sistema tiene $k$ ligaduras, se podrán introducir $n=3 N-k$, coordenadas independientes $q=\left(q_{1}, \ldots, q_{n}\right)$ de forma que podremos expresar las posiciones de las partículas como

$$
\mathbf{x}_{i}=\mathbf{x}_{i}(\mathbf{q}, t), i=1, \ldots, N
$$


Este conjunto de coordenadas independientes se llamarán coordenadas generalizadas, y al espacio $n$-dimensional de las coordenadas libres le llamaremos espacio de configuración. Se llamará número de grados de libertad al número $n$ de coordenadas libres del sistema. Las derivadas de las coordenadas generalizadas o velocidades generalizadas se indican por

$$
\dot{\mathbf{q}}=\left(\dot{q}_{1}, \ldots, \dot{q_{n}}\right)
$$

Se llama energía cinética a la función

$$
T=\sum_{i=0}^{N} \frac{1}{2} m_{i}{\dot{x_{i}}}^{2}
$$

y puede expresarse en función de las coordenadas y velocidades generalizadas, ya que $\mathbf{x}_{i}=\mathbf{x}_{i}\left(q_{1}, \ldots, q_{n}, t\right) \mathrm{y}$

$$
\dot{\mathbf{x}}_{\mathbf{i}}=\frac{\partial \mathbf{x}_{i}}{\partial \mathbf{q}} \cdot \frac{d \mathbf{q}}{d t}+\frac{\partial x}{\partial t}=\phi_{i}(\mathbf{q}, \dot{\mathbf{q}}, \mathbf{t}) .
$$

Por lo que al final la energía cinética resulta ser una función de $\mathbf{q}, \dot{\mathbf{q}}, t$, es decir, $T=$ $T(\mathbf{q}, \dot{\mathbf{q}}, \mathbf{t})$. Si la resultante $\mathbf{F}_{i}$ de las fuerzas que actúan sobre cada partícula $P_{i}$ puede obtenerse como

$$
\mathbf{F}_{i}=-\nabla_{x_{i}} V
$$

a la función $V$ se le llama la energía potencial del sistema y se dice que el sistema es conservativo.

En general, $V$ depende de $\mathbf{q}$ y $t$, es decir, $V=V(\mathbf{q})$ o $V=V(\mathbf{q}, t)$.

\subsection{Ecuaciones de Lagrange y de Hamilton}

Llamaremos función lagrangiana de un sistema dinámico consevativo como el anterior, a la expresión

$$
\mathcal{L}(\mathbf{q}, \dot{\mathbf{q}}, t)=T(\mathbf{q}, \dot{\mathbf{q}}, t)-V(\mathbf{q}, t),
$$

es decir, a la diferencia entre la energía cinética y la energía potencial del sistema.

Las ecuaciones del movimiento (2.1) pueden expresarse en términos de la función lagrangiana

$$
\frac{d}{d t}\left(\frac{\partial \mathcal{L}}{\partial \dot{\mathbf{q}}_{i}}\right)-\frac{\partial \mathcal{L}}{\partial \mathbf{q}_{i}}=0, i=1, \ldots, n
$$

que equivale a

$$
\frac{d}{d t}\left(\nabla_{\dot{\mathbf{q}}_{\mathbf{i}}} \mathcal{L}\right)-\nabla_{q_{i}} \mathcal{L}=0, i=1, \ldots, n
$$

Estas ecuaciones se llamarán ecuaciones de Lagrange y son equivalentes a las ecuaciones de Newton del sistema.

Definimos ahora los momentos generalizados $\mathbf{p}=\left(p_{1}, \ldots, p_{n}\right)$ en la forma

$$
p_{i}=\frac{\partial \mathcal{L}(\mathbf{q}, \dot{\mathbf{q}}, t)}{\partial \dot{q}_{i}},(i=1, \ldots, n)
$$

Estas ecuaciones se suponen inversibles con respecto a $\dot{\mathbf{q}}$ obteniéndose

$$
\dot{q}_{i}=\dot{q}_{i}(\mathbf{q}, \mathbf{p}, t)(i=1, \ldots, n)
$$


$\mathrm{Al}$ espacio $2 n$ dimensional (q, p) le llamaremos espacio fásico (o espacio de fases) y función hamiltoniana o hamiltoniano $\mathcal{H}$ a la transformada de Legendre de la función Lagrangiana dada por la fórmula

$$
\mathcal{H}(\mathbf{q}, \mathbf{p}, t)=\mathbf{p} \cdot \dot{\mathbf{q}}(\mathbf{q}, \mathbf{p}, t)-\mathcal{L}(\mathbf{q}, \dot{\mathbf{q}}(\mathbf{q}, \mathbf{p}, t), t)
$$

donde $\mathbf{q}=\left(q_{1}, \ldots, q_{n}\right) \in U \subset \mathbb{R}^{n}, \mathbf{p}=\left(p_{1}, \ldots, p_{n}\right) \in \mathbb{R}^{n}$ y $U$ es un subconjunto abierto de $\mathbb{R}^{n}$ y $\mathcal{H}$ es una función de clase $C^{r}$ donde $r \geq 2$.

El sistema de $n$ ecuaciones diferenciales de segundo orden de Lagrange (2.3) es entonces equivalente al sistema de $2 n$-ecuaciones de primer orden

$$
\dot{\mathbf{q}}=\frac{\partial \mathcal{H}}{\partial \mathbf{p}}=\nabla_{\mathbf{p}} \mathcal{H} \quad \dot{\mathbf{p}}=-\frac{\partial \mathcal{H}}{\partial \mathbf{q}}=-\nabla_{\mathbf{q}} \mathcal{H}
$$

llamadas ecuaciones de Hamilton o canónicas del sistema.

También podemos utilizar de manera más compacta $\mathbf{x}=(\mathbf{q}, \mathbf{p}) \in U \times \mathbb{R}^{n}$, con $U$ abierto de $\mathbb{R}^{n}$, que es un vector de coordenadas y momentos. De esta forma el hamiltoniano quedaría expresado como

$$
\mathcal{H}(\mathbf{x}, t)=\mathcal{H}(\mathbf{q}, \mathbf{p}, t)
$$

La evolución dinámica del sistema viene dada por las ecuaciones de Hamilton que en forma compacta se escriben como sigue

$$
\dot{\mathbf{x}}=\mathcal{J} \nabla_{x} \mathcal{H}
$$

donde $\mathcal{J}$ es la matriz antisimétrica

$$
\mathcal{J}=\left(\begin{array}{cc}
0_{n} & I_{n} \\
-I_{n} & 0_{n}
\end{array}\right)
$$

que verifica que $\mathcal{J}^{-1}=\mathcal{J}^{T}=-\mathcal{J}$, siendo $0_{n}$ la matriz nula de orden $n$, e $I_{n}$ la matriz identidad de orden $n$.

Asociado al sistema (2.6) encontramos el campo vectorial hamiltoniano

$$
X_{\mathcal{H}}=\left(\frac{\partial \mathcal{H}}{\partial \mathbf{p}},-\frac{\partial \mathcal{H}}{\partial \mathbf{q}}\right): U \times \mathbb{R}^{n} \rightarrow \mathbb{R}^{2 n}
$$

\subsection{Transformaciones canónicas y funciones genera- trices}

Las transformaciones que suponen el cambio de unas coordenadas a otras, como por ejemplo, pasar de coordenadas cartesianas a coordenadas polares planas, reciben el nombre de transformaciones puntuales. Ahora bien, en la formulación hamiltoniana, los momentos generalizados, como ya hemos visto, son también variables independientes al mismo nivel que las coordenadas generalizadas. Por tanto, el concepto de transformación de coordenadas, debe ampliarse para que incluya la transformación simultánea de coordenadas y momentos $q_{i}, p_{i}$, a un nuevo sistema $Q_{i}, P_{i}$.

Las transformaciones canónicas pueden utilizarse para proporcionar un método general de resolución de problemas mecánicos. 
Dada una aplicación $\psi$ definida en un subconjunto abierto de $\mathbb{R}^{2 n+1}$ de la forma

$$
(t, \mathbf{x}) \longrightarrow^{\psi} \mathbf{y}
$$

definida por las expresiones $\mathbf{y}=\mathbf{y}(\mathbf{x}, t)$, que supondremos de clase $C^{1}$, donde $\mathbf{x}=(\mathbf{q}, \mathbf{p})$ e $\mathbf{y}=(\mathbf{Q}, \mathbf{P})$ son las coordenadas y momentos antiguos y nuevos.

Una transformación de este tipo se dice canónica si y solo si existe una constante $\mu \neq 0$ tal que se satisface la $\Gamma \mathcal{J} \Gamma^{T}=\mu \mathcal{J}(*)$, donde $\Gamma$ es la matriz Jacobiana

$$
\Gamma=\mathbf{y}_{x}=\nabla_{\mathbf{x}} \mathbf{y}=\left(\frac{\partial y_{i}}{\partial x_{j}}\right)=\left(\begin{array}{ccc}
\frac{\partial y_{1}}{\partial x_{1}} & \cdots & \frac{\partial y_{1}}{\partial x_{2 n}} \\
\cdots & \cdots & \cdots \\
\frac{\partial y_{2 n}}{\partial x_{1}} & \cdots & \frac{\partial y_{2 n}}{\partial x_{2 n}}
\end{array}\right)
$$

siendo el $\operatorname{det}(\Gamma) \neq 0$ en el dominio $(\mathbf{x}, t)$ que se considere.

Las matrices que cumplen la relación $(*)$ se dicen simplécticas. A $\mu$ se le llama multiplicador de la transformación. En particular, si $\mu=1$ la transformación se llama complétamente canónica (t.c.c).

Veamos algunas de las propiedades de estas transformaciones.

Proposición 1 El conjunto de las transformaciones canónicas forma grupo con respecto a la operación de composición de transformaciones. Las transformaciones completamente canónicas forman un subgrupo del grupo anterior.

Observación 2 De esta forma, para cada t.c.c $\psi$, con $\mathbf{y}=\mathbf{y}(\mathbf{x}, t)$ existe una transformación inversa $\varphi$, con $\mathbf{x}=\mathbf{x}(\mathbf{y}, t)$. Esta transformación puede ser aplicada a la función $F(\mathbf{x}, t)$ definida en el espacio fásico con lo que obtendremos la función transformada $\varphi^{*} F(\mathbf{y}, t)=F(\mathbf{x}(\mathbf{y}, t), t)$.

Proposición 3 Una transformación $y=y(x, t)$ es canónica si y solo si transforma todo sistema hamiltoniano $\dot{x}=\mathcal{J H}_{x}$ en otro del mismo tipo $\dot{y}=J \mathcal{K}_{y}$, siendo el nuevo hamiltoniano

$$
\mathcal{K}(y, t)=\mu \mathcal{H}(x(y, t), t)+R(y, t)
$$

$\operatorname{con} \mathcal{R}_{y}=J y_{t}$

En particular, si la transformación no depende del tiempo, puede tomarse $\mathcal{R}=0$, si además fuese $\mu=1$ (se trataría de una tranformación completamente canónica e independiente del tiempo) entonces

$$
\mathcal{K}(y, t)=\mathcal{H}(x(y, t), t)
$$

es decir, el nuevo hamiltoniano sería el antiguo expresado en las nuevas variables.

Proposición 4 Una transformación $\mathbf{y}=\mathbf{y}(\mathbf{x}, t)$ es canónica si y solo si existen funciones $\mathcal{W}$ y $\mathcal{R}$, tal que

$$
d \mathcal{W}=2 \mathcal{R} d t-\mu \mathbf{x} \cdot \mathcal{J} d \mathbf{x}+\mathbf{y} \cdot \mathcal{J} d \mathbf{y}
$$

siendo $\mu$ constante $y \mathcal{R}_{y}=\mathcal{J} y_{t}$.

Para el caso de t.c.c. independientes del tiempo se tiene el siguiente resultado 
Proposición 5 Una transformación $\mathbf{y}=\mathbf{y}(\mathbf{x})$ es completamente canónica si y solo si existe una función $\mathcal{W}$ tal que se verifica una cualquiera de las relaciones siguientes:

$$
\begin{aligned}
& d \mathcal{W}=\mathbf{q} \cdot d \mathbf{p}+\mathbf{P} \cdot d \mathbf{Q} \\
& d \mathcal{W}=\mathbf{q} \cdot d \mathbf{p}-\mathbf{Q} \cdot d \mathbf{P} \\
& d \mathcal{W}=\mathbf{p} \cdot d \mathbf{q}+\mathbf{Q} \cdot d \mathbf{P} \\
& d \mathcal{W}=\mathbf{p} \cdot d \mathbf{q}-\mathbf{P} \cdot d \mathbf{Q}
\end{aligned}
$$

Proposición 6 Sean $S^{(1)}(\mathbf{P}, \mathbf{q}, t), S^{(2)}(\mathbf{p}, \mathbf{Q}, t), S^{(3)}(\mathbf{p}, \mathbf{P}, t), S^{(4)}(\mathbf{q}, \mathbf{Q}, t)$ funciones de clase $C^{2}$ tales que $\operatorname{det}\left(S_{\mathbf{P q}}^{(1)}\right) \neq 0, \operatorname{det}\left(S_{\mathbf{p Q}}^{(2)}\right) \neq 0, \operatorname{det}\left(S_{\mathbf{p P}}^{(3)}\right) \neq 0, \operatorname{det}\left(S_{\mathbf{q} \mathbf{Q}}^{(4)}\right) \neq 0$, entonces las ecuaciones

$$
\begin{array}{llll}
\mathbf{p}=\nabla_{\mathbf{q}} S^{(1)}, & \mathbf{Q}=\nabla_{\mathbf{P}} S^{(1)}, & \mathcal{R}^{(1)}=S_{t}^{(1)}, \\
\mathbf{q}=-\nabla_{\mathbf{p}} S^{(2)}, & \mathbf{P}=-\nabla_{\mathbf{Q}} S^{(2)}, & \mathcal{R}^{(2)}=S_{t}^{(2)}, \\
\mathbf{q}=-\nabla_{\mathbf{P}} S^{(3)}, & \mathbf{Q}=\nabla_{\mathbf{P}} S^{(3)}, & \mathcal{R}^{(3)}=S_{t}^{(3)}, \\
\mathbf{p}=\nabla_{\mathbf{q}} S^{(4)}, & \mathbf{P}=-\nabla_{\mathbf{Q}} S^{(4)}, & \mathcal{R}^{(4)}=S_{t}^{(4)}
\end{array}
$$

definen transformaciones completamente canónicas de función resto $\mathcal{R}^{(i)}$. A las funciones $S^{(i)}$ se les llama función generatriz (o generador) de la transformación, y a las transformaciones generadas se les llama transformaciones de contacto.

Se puede ver en el siguiente teorema cómo obtener las transformaciones canónicas a través de funciones generatrices.

Teorema 7 Para una elección arbitraria de funciones generatrices $\mathcal{S}^{(k)}$ de la lista (2.10), obtendremos una transformación completamente canónica que modifica el hamiltoniano (autónomo o no) añadiendo el término $\mathcal{R}^{(k)}=\partial \mathcal{S}^{(k)} / \partial t$, es decir,

$$
\mathcal{K}=\mathcal{H}+\mathcal{R}^{(k)}
$$

donde $\mathcal{H}$ y $\mathcal{R}^{(k)}$ se suponen expresadas en las nuevas variables $\mathbf{Q}, \mathbf{P}$

Se observa en el teorema previo que si la función generatriz $S^{(k)}$ no depende del tiempo, entonces la transformación canónica definida implícitamente por ella deja el hamiltoniano invariante, es decir, el nuevo hamiltoniano es el antiguo expresado en las nuevas variables.

\subsection{Ecuación de Hamilton-Jacobi}

Sea ahora una función generartiz $S(\mathbf{P}, \mathbf{q}, t)$, del tipo $S^{(1)}$ (véase(2.10)) de una transformación completamente canónica que satisface la ecuación de Hamilton-Jacobi

$$
\mathcal{H}\left(\mathbf{q}, \nabla_{\mathbf{q}} S, t\right)+S_{t}=0
$$

entonces las nuevas variables y momentos $(\mathbf{Q}, \mathbf{P})$ son constantes (integrales) del sistema dinámico de hamiltoniano $\mathcal{H}$.

Si $\mathcal{H}$ no es una función explícita de $t$, la ecuación de Hamilton-Jacobi para $S$ adoptará la forma

$$
\mathcal{H}\left(\mathbf{q}, \nabla_{\mathbf{q}} S\right)+S_{t}=0
$$


donde el segundo término contiene solamente la dependencia de $t$, mientras que el primero sólo se refiere a la dependencia de $S$ respecto a q. Así pues, en el caso en que el hamiltoniano sea conservativo se tendrá

$$
\mathcal{H}\left(\mathbf{q}, \nabla_{\mathbf{q}} S\right)=-S_{t}=P_{1}
$$

donde $P_{1}$ es una constante que suele tomarse como nuevo primer momento. De esta forma

$$
S=-P_{1} t+W(\mathbf{P}, \mathbf{q})
$$

y la ecuación de Hamilton-Jacobi se transforma en

$$
\mathcal{H}\left(\mathbf{q}, \nabla_{\mathbf{q}} W\right)=P_{1}
$$

Encontrar una función $W$, independiente del tiempo, solución de la ecuación anterior equivale a encontrar una transformación canónica que transforma el hamiltoniano en $\mathcal{K}(\mathbf{Q}, \mathbf{P})=P_{1}$, es decir, el hamiltoniano ha de ser igual al nuevo primer momento $P_{1}$. Esta función $W$, se llama función característica de Hamilton, y genera una nueva transformación canónica en la cual todas las coordenadas nuevas son cíclicas a excepción de la primera, siendo por tanto $Q_{1}=t+Q_{1}^{0}$, lineal en el tiempo y las restantes constantes $Q_{i}=Q_{i}^{0}(i=2, \ldots, n)$, en tanto que los nuevos momentos son todos constantes $P_{i}=P_{i}^{0}(i=1,2, \ldots, n)$. Para resolver (2.13) utilizaremos, cuando sea posible, el método de separación de las variables.

\subsection{Teoremas de Liouville y Liouville-Arnold}

Sea $\left(\mathbf{q}_{0}, \mathbf{p}_{0}\right) \in U \times \mathbb{R}^{n}$ y sea también la solución del sistema $(2.6) \phi_{t}^{\mathcal{H}}\left(\mathbf{q}_{0}, \mathbf{p}_{0}\right)$, donde $\phi_{0}^{\mathcal{H}}\left(\mathbf{q}_{0}, \mathbf{p}_{0}\right)=\left(\mathbf{q}_{0}, \mathbf{p}_{0}\right)$. Entonces el intervalo $I_{\left(\mathbf{q}_{0}, \mathbf{p}_{0}\right)}$ de definición de la solución $\phi_{t}^{\mathcal{H}}\left(\mathbf{q}_{0}, \mathbf{p}_{0}\right)$ es maximal si para cada solución $\psi_{t}^{\mathcal{H}}\left(\mathbf{q}_{0}, \mathbf{p}_{0}\right)$ del sistema (3.1) definido en el intervalo $J$ tal que $\psi_{0}^{\mathcal{H}}\left(\mathbf{q}_{0}, \mathbf{p}_{0}\right)=\left(\mathbf{q}_{0}, \mathbf{p}_{0}\right), J \subset I_{\left(\mathbf{q}_{0}, \mathbf{p}_{0}\right)}$ y $\left.\phi_{t}^{\mathcal{H}}\right|_{J}=\psi_{t}^{\mathcal{H}}$. Una solición $\phi_{t}^{\mathcal{H}}\left(\mathbf{q}_{0}, \mathbf{p}_{0}\right)$ es maximal si su intervalo de definición es maximal. Por tanto, la solución maximal $\phi_{t}^{\mathcal{H}}\left(\mathbf{q}_{0}, \mathbf{p}_{0}\right)$ es la función $I_{\left(\mathbf{q}_{0}, \mathbf{p}_{0}\right)} \rightarrow U \times \mathbb{R}^{n}$ definida por $t \rightarrow \phi_{t}^{\mathcal{H}}\left(\mathbf{q}_{0}, \mathbf{p}_{0}\right)$.

El subconjunto $\left\{\phi_{t}^{\mathcal{H}}\left(\mathbf{q}_{0}, \mathbf{p}_{0}\right): t \in I_{\left(\mathbf{q}_{0}, \mathbf{p}_{0}\right)}\right\}$ de $U \times \mathbb{R}^{n}$ es llamado la órbita del sistema (2.6) asociada a la solución $t \rightarrow \phi_{t}^{\mathcal{H}}\left(\mathbf{q}_{0}, \mathbf{p}_{0}\right)$.

El flujo $\phi_{t}^{\mathcal{H}}$ asociado al campo vectorial $X_{\mathcal{H}}$ es la aplicación

$$
\bigcup_{\left(\mathbf{q}_{0}, \mathbf{p}_{0}\right) \in U \times \mathbb{R}^{n}} I_{\left(\mathbf{q}_{0}, \mathbf{p}_{0}\right)} \times\left\{\left(\mathbf{q}_{0}, \mathbf{p}_{0}\right)\right\} \rightarrow U \times \mathbb{R}^{n}
$$

tal que

$$
\left(t,\left(\mathbf{q}_{0}, \mathbf{p}_{0}\right)\right) \rightarrow \phi_{t}^{\mathcal{H}}\left(\mathbf{q}_{0}, \mathbf{p}_{0}\right)
$$

El flujo de (2.6) es completo si todas sus soluciones están definidas para todo $t \in \mathbb{R}$, es decir, el intervalo maximal de definición de todas las soluciones es $\mathbb{R}$.

Un subconjunto $V \subset U \times \mathbb{R}^{n}$ es invariante para el flujo si para cada órbita que tiene un punto en $V$ está enteramente contenida en $V$.

El corchete de Poisson de dos funciones de clase $C^{1}, \mathcal{F}$ y $\mathcal{G}$ de $U \times \mathbb{R}^{n}$ en $\mathbb{R}$ es definido como la función $\{\mathcal{F}, \mathcal{G}\}: U \times \mathbb{R}^{n} \rightarrow \mathbb{R}$ dada por

$$
\{\mathcal{F}, \mathcal{G}\}=\left(\frac{\partial \mathcal{F}}{\partial \mathbf{q}}\right) \cdot\left(\frac{\partial \mathcal{G}}{\partial \mathbf{p}}\right)-\left(\frac{\partial \mathcal{F}}{\partial \mathbf{p}}\right) \cdot\left(\frac{\partial \mathcal{G}}{\partial \mathbf{q}}\right)=\sum_{i=1}^{n}\left(\frac{\partial \mathcal{F}}{\partial q_{i}} \frac{\partial \mathcal{G}}{\partial p_{i}}-\frac{\partial \mathcal{F}}{\partial p_{i}} \frac{\partial \mathcal{G}}{\partial q_{i}}\right)
$$


donde el punto se refiere al producto escalar euclídeo.

Se dice que $n$ funciones de clase $C^{1}, \mathcal{F}_{1}, \ldots, \mathcal{F}_{n}$ de $U \times \mathbb{R}^{n}$ en $\mathbb{R}$ están en involución si $\left\{\mathcal{F}_{i}, \mathcal{F}_{j}\right\}=0$ para cada $i, j \in\{1, \ldots, n\}$, y que son independientes si las 1 - formas $d \mathcal{F}_{1}, \ldots, d \mathcal{F}_{n}$ son linealmente independientes en un subconjunto de $U \times \mathbb{R}^{n}$ de medida de Lebesgue completa.

Una función de clase $C^{1}$ no-constante $\mathcal{F}$ de $U \times \mathbb{R}^{n}$ en $\mathbb{R}$ que satisface $\{\mathcal{F}, \mathcal{H}\}=0$ es llamada integral primera del sistema 2.6 o del campo $X_{\mathcal{H}}$. En particular, para todo hamiltoniano autónomo $\mathcal{H}$ es una integral primera del sistema. Es fácil verificar que si $\phi_{t}^{\mathcal{H}}\left(\mathbf{q}_{0}, \mathbf{p}_{0}\right)$ es una solución del sistema 2.6 y $F$ es una integral primera entonces $F\left(\phi_{t}^{\mathcal{H}}\left(\mathbf{q}_{0}, \mathbf{p}_{0}\right)\right)$ es constante, es decir, es independiente de $t$.

Definición 8 Un sistema hamiltoniano con $n$ grados de libertad se dice integrable si tiene $n$ itegrales primeras independientes en involución.

Claramente sabemos que todas las órbitas de un sistema hamiltoniano autónomo de 1 grado de libertad, están dadas por las curvas $\mathcal{H}(q, p)=$ siendo $c$ una constante arbitraria.

Una pregunta que es natural hacerse es la siguiente: ¿Para qué sistemas hamiltonianos de 2 o más grados de libertad, podemos saber todas sus soluciones?

La mejor respuesta hasta ahora a dicha pregunta nos la dió Liouville.

Teorema 9 (Liouville) Para un sistema hamiltoniano integrable, sus soluciones se pueden calcular mediante cuadraturas.

Este teorema de carácter claramente analítico, recoge casi todos los problemas de dinámica resueltos hasta la fecha. Arnold mejoró su resultado añadiéndole la geometría de los sistemas hamiltonianos integrables.

Pero antes de describir la versión de Arnold del teorema de Liouville necesitamos introducir más notación.

Ahora nos referimos a $U$ como un subconjunto abierto de $\mathbb{R}^{m}$ y empezaremos introduciendo la noción de regularidad. Sea $\mathbf{f}=\left(f_{1}, \ldots, f_{n}\right)$ un aplicación de clase $C^{1}$ de $U$ a $\mathbb{R}^{n}$. Decimos que $\mathbf{x} \in U$ es un punto regular si $f_{1}, \ldots, f_{n}$ son independientes en $\mathbf{x}$ (esto es, las diferenciales $\left(d f_{1}\right)(\mathbf{x}), \ldots,\left(d f_{n}\right)(\mathbf{x})$ son linealmente independientes o simplemente la aplicación lineal $d \mathbf{f}(\mathbf{x})$ es suprayectiva). Un punto $\mathbf{x}$ que no es regular es llamado punto crítico.

Un punto $\mathbf{y} \in \mathbb{R}^{n}$ es un valor regular para $\mathbf{f}$ si $f_{1}, \ldots, f_{n}$ son independientes para todo $\mathbf{x} \in U$ tal que $\mathbf{f}(\mathbf{x})=\mathbf{y}$. Si $\mathbf{y}$ no es un valor regular, lo llamaremos valor crítico. Por lo tanto, los valores regulares son aquellos cuya imagen inversa por $\mathbf{f}$ está formada por puntos regulares o está vacía, y los valores críticos son la imagen de algún punto crítico (o aquellos cuya imagen inversa por $\mathbf{f}$ contiene un punto crítico de $\mathbf{f}$ ).

Si denotamos al conjunto de todos los puntos críticos de $\mathbf{f}$ por $\bar{\sigma}_{\mathbf{f}}$, y al conjunto de todos sus valores críticos por $\sigma_{\mathbf{f}}$, entonces $\sigma_{\mathbf{f}}=\mathbf{f}\left(\bar{\sigma}_{\mathbf{f}}\right)$. Desde el teorema de Sard se sigue que $\mathbb{R}^{n} \backslash \sigma_{\mathbf{f}}$ tiene medida de Lebesgue completa.

Para $\mathbf{y} \in \mathbb{R}^{n}$ denotamos por $I_{\mathbf{y}}$ al conjunto $\mathbf{f}^{-1}(\mathbf{y})=\{\mathbf{x} \in U: \mathbf{f}(\mathbf{x})=\mathbf{y}\}$.

Se dice que $I_{\mathbf{y}}$ cambia su topología en $\mathbf{c} \in \mathbf{f}(U)$ o simlemente que la topología de $I_{\mathbf{c}}$ cambia si para cada entorno $V$ de $\mathbf{c}$ existe $\mathbf{y} \in V$ tal que $I_{\mathbf{y}}$ y $I_{\mathbf{c}}$ no son homeomorfos. En otro caso, decimos que la topología de $I_{\mathbf{c}}$ no cambia. Así pues, si la topología de $I_{\mathbf{c}}$ no cambia, será la misma en algún entorno $V$ de c, y por tanto, es equivalente a decir que la topología de $I_{\mathbf{y}}$ no cambia para cada $\mathbf{y} \in V$. 
Sea

$$
T^{k}=\mathbb{R}^{k} / \mathbb{Z}^{k} \approx S^{1} \times \cdots \times S^{1}
$$

el toro $k$-dimensional . Una cilindro generalizado lineal $T^{k} \times \mathbb{R}^{n-k}$ es una aplicación lineal $\varphi: \mathbb{R} \times T^{k} \times \mathbb{R}^{n-k} \rightarrow T^{k} \times \mathbb{R}^{n-k}$ tal que

$$
\varphi(t, \mathbf{z})=\left(z_{1}+t v_{1}(\bmod 1), \ldots, z_{k}+t v_{k}(\bmod 1), z_{k+1}+t v_{k+1}, \ldots, z_{n}+t v_{n}\right),
$$

donde $t \in \mathbb{R},\left(z_{1}, \ldots, z_{k}\right) \in T^{k},\left(z_{k+1}, \ldots, z_{n}\right) \in \mathbb{R}^{n-k}$ y $\left(v_{1}, \ldots, v_{n}\right) \in \mathbb{R}^{n}$.

Se dice que el vector de campo $X_{\mathcal{H}_{1}}$, definido en $U_{1} \times \mathbb{R}^{n}$ que es un subconjunto abierto de $\mathbb{R}^{2 n}$, es topológicamente conjugado (o simplemente conjugado) para el vector de campo $X_{\mathcal{H}_{2}}$, definido en $U_{2} \times \mathbb{R}^{n}$ que es un subconjunto abierto de $\mathbb{R}^{2 n}$, si existe un homeomorfismo h : $U_{1} \times \mathbb{R}^{n} \rightarrow U_{2} \times \mathbb{R}^{n}$ tal que

$$
\left(\mathbf{h} \circ \varphi_{t}^{\mathcal{H}_{1}}\right)(\mathbf{z})=\left(\varphi_{t}^{\mathcal{H}_{2}} \circ \mathbf{h}\right)(\mathbf{z}),
$$

para todo $t$ donde las soluciones $\varphi_{t}^{\mathcal{H}_{1}}(\mathbf{z})$ y $\varphi_{t}^{\mathcal{H}_{2}}(\mathbf{h}(\mathbf{z}))$ están definidos para todo $\mathbf{z} \in U_{1} \times \mathbb{R}^{n}$. Esto es, $\mathbf{h}$ lleva órbitas de $X_{\mathcal{H}_{1}}$ a órbitas de $X_{\mathcal{H}_{2}}$ manteniendo la orientación de las órbitas y el parámetro $t$. Si $\mathbf{h}$ es de clase $C^{r}$, diremos que estos campos vectoriales son $C^{r}$ conjugados.

Teorema 10 (Liouville-Arnold) Consideremos un sistema hamiltoniano integrable $X_{\mathcal{H}}$ con $n$ grados de libertad definido por el $C^{2}$ hamiltoniano $H: U \times \mathbb{R}^{n} \rightarrow \mathbb{R}$, donde $U \times \mathbb{R}^{n}$ es un subconjunto abierto de $\mathbb{R}^{2 n}$ y sean $\mathcal{H}, \mathcal{F}_{1}, \ldots, \mathcal{F}_{n-1}, n$ integrales primeras independientes en involución. Si $I_{h, f_{1}, \ldots, f_{n-1}} \neq \emptyset y\left(h, f_{1}, \ldots, f_{n-1}\right)$ es un valor regular de $\left(\mathcal{H}, \mathcal{F}_{1}, \ldots, \mathcal{F}_{n-1}\right)$, entonces las siguientes afirmaciones son ciertas. $X_{\mathcal{H}}$.

a) $I_{h, f_{1}, \ldots, f_{n-1}}$ es un $n$-dimensional subvariendad de $U \times \mathbb{R}^{n}$ invariante bajo el flujo de

b) Si el flujo en una componente conexa $I_{h, f_{1}, \ldots, f_{n-1}}^{*}$ de $I_{h, f_{1}, \ldots, f_{n-1}}$ es completo, entonces $I_{h, f_{1}, \ldots, f_{n-1}}^{*}$ es difeomorfo a $T^{k} \times \mathbb{R}^{n-k}$ para algún $k \in\{0, \ldots, n\}$. Notemos que si $I_{h, f_{1}, \ldots, f_{n-1}}^{*}$ es compacta, entonces el flujo en ella es siempre completo.

c) Bajo la hipótesis b), el flujo en $I_{h, f_{1}, \ldots, f_{n-1}}^{*}$ es conjugado a un flujo lineal en $T^{k} \times \mathbb{R}^{n-k}$.

De hecho, el anterior teorema es una versión mejorada del teorema original de Arnold. Para más detalles sobre sistemas hamiltonianos y la prueba del teorema de LiouvilleArnold véase $[1,3,4]$.

Como se ha comentado anteriormente, una característica importante del estudio de sistemas hamiltonianos integrables, es describir la topología de los subconjuntos invariantes $E_{h}, I_{h, f_{1}} \ldots, I_{h, f_{1}, \ldots, f_{n-1}}$ del espacio fásico. Más precisamente, ¿cómo es la foliación del espacio fásico por medio de los niveles de energía $E_{h}$ ? ¿Cómo es $E_{h}$ foliada por $I_{h, f_{1}}$ ? ¿Cómo $I_{h, f_{1}}$ es foliada por $I_{h, f_{1}, f_{2}}$ ? Y así sucesivamente.

El teorema de Liouville-Amold nos ayuda a contestar las preguntas previas pero éstas no se pueden resolver. Es más, este teorema solo establece la topología de las variedades $n$ dimensionales conexas $I_{h, f_{1}, \ldots, f_{n-1}}^{*}$ cuando $\left(h, f_{1}, \ldots, f_{n-1}\right)$ es un valor regular y cuando el flujo asociado correspondiente es completo.

Por supuesto el conjunto $\left\{\left(h, f_{1}, \ldots, f_{n-1}\right):\left(h, f_{1}, \ldots, f_{n-1}\right)\right.$ es un valor regular\} tiene medida completa en $\mathbb{R}^{n}$, pero en general es diferente de $\mathbb{R}^{n}$. Por tanto, es también necesario describir los subconjuntos $I_{h, f_{1}, \ldots, f_{n-1}}$ asociados a los valores críticos $\left(h, f_{1}, \ldots, f_{n-1}\right)$, y al flujo sobre ellos. 


\subsection{Elementos de mecánica-geométrica}

\subsubsection{Introducción y terminología}

En general, el espacio de configuración de un sistema mecánico langragiano será una variedad diferenciable $n$-dimensional $\mathbf{M}$ que supondremos de clase $C^{\infty}$, cuyas coordenadas denotaremos por $q=\left(q_{1}, \cdots, q_{n}\right)$; en tanto que la función lagrangiana, en el caso autónomo, estará definida sobre el fibrado tangente $\mathbf{T} \mathbf{M}=\cup_{m \in \mathbf{M}} \mathbf{T}_{\mathbf{m}} \mathbf{M}$ (unión disjunta de los espacios tangente a cada $m \in \mathbf{M}$ ) en la forma

$$
\begin{aligned}
\mathcal{L}: \mathbf{T M} & \longrightarrow \mathbb{R} \\
(q, \dot{q}) & \longrightarrow \mathcal{L}(q, \dot{q})
\end{aligned}
$$

Es fácil ver que TM es una variedad diferenciable $2 n$ dimensional.

Las coordenadas sobre $\mathbf{T M}$ se expresan en la forma $(q, \dot{q})$, donde $\dot{q}=\left(\dot{q}_{1}, \cdots, \dot{q}_{n}\right)$ es la velocidad generalizada que es un vector tangente a la variedad de configuración $\mathbf{M}$.

Asimismo, se define el fibrado cotangente a la variedad $\mathbf{M}$ como $\mathbf{T}^{*} \mathbf{M}=\cup_{m \in \mathbf{M}} \mathbf{T}^{*} \mathbf{M}$ (es la unión disjunta de los $\mathbf{T}_{\mathbf{m}}^{*} \mathbf{M}=\left\{\omega_{m}: \mathbf{T}_{\mathbf{m}} \mathbf{M} \longrightarrow \mathbb{R} \mid \omega_{m}\right.$ es lineal $\}$, a cuyos elementos llamaremos covectores o 1-formas, los espacios vectoriales $\mathbf{T}_{\mathbf{m}}^{*} \mathbf{M}$ tienen la misma dimensión $n$ que los $\mathbf{T}_{\mathbf{m}} \mathbf{M}$. La acción natural de un vector cotangente $\omega_{m} \in \mathbf{T}_{\mathbf{m}}^{*} \mathbf{M}$ sobre un vector tangente $X_{m} \in \mathbf{T}_{\mathbf{m}} \mathbf{M}$ se escribe como $\left\langle\omega_{m}, X_{m}\right\rangle$. Si $\left\{\frac{\partial}{\partial q_{i}} \mid 1=1, \cdots, n\right\}$ es una base de $\mathbf{T}_{\mathbf{m}} \mathbf{M}$, la base dual para $\mathbf{T}_{\mathbf{m}}^{*} \mathbf{M}$ está dada por $\left\{d q_{i} \mid 1=1, \cdots, n\right\}$ donde $\left\langle d q_{i}, \frac{\partial}{\partial q_{i}}\right\rangle=\delta_{i j}, i, j=1, \cdots, n$.

$\mathbf{T}^{*} \mathbf{M}$ es también una variedad diferenciable $2 n$-dimensional, que suele denominarse espacio o variedad de fase, y sus elementos se escriben en la forma $(q, p)=\left(q_{1}, \cdots, q_{n}, p_{1, \cdots}, p_{n}\right)$.

Para un lagrangiano $L$ tal que el hessiano $\left\|\frac{\partial^{2} L}{\partial \dot{q}_{i} \partial \dot{q}_{j}}\right\| \neq 0$, se define la transformada de Legendre (o fiber derivative) en la forma

$$
\begin{aligned}
\mathbb{F} L: & \mathbf{T M} \longrightarrow \mathbf{T}^{*} \mathbf{M} \\
\left(q_{i}, \dot{q}\right) & \longrightarrow\left(q_{i}, p_{j}\right)
\end{aligned}
$$

con $p_{j}=\frac{\partial L}{\partial \dot{q}_{j}}$.

Un lagrangiano se dice hiperregular si $\mathbb{F} L$ es un difeomorfismo; en este caso se define el hamiltoniano correspondiente por

$$
\mathcal{H}\left(q_{i}, p_{i}\right)=\sum_{i=1}^{n} p_{i} \dot{q}-L
$$

siendo las ecuaciones de Lagrange para $L$ equivalentes a las de Hamilton para $\mathcal{H}$.

Observación 11 En el caso no autónomo la lagrangiana será una función $\mathcal{L}: \mathbf{T M} \times$ $\mathbb{R} \longrightarrow \mathbb{R}$ y la hamiltoniana $\mathcal{H}: \mathbf{T}^{*} \mathbf{M} \times \mathbb{R} \longrightarrow \mathbb{R}$. 


\subsubsection{Notaciones usuales}

$\mathcal{F}(\mathbf{M})=\left\{f: \mathbf{M} \longrightarrow \mathbb{R} \mid\right.$ donde $f$ es de clase $\left.C^{\infty}\right\}$.

Un campo vectorial $X$ sobre una variedad $\mathbf{M}$ es una aplicación $C^{\infty}, X: \mathbf{M} \longrightarrow \mathbf{T M}$, tal que $\pi \circ X=i d_{\mathbf{M}}$, donde $\pi$ es la proyección canónica de $\mathbf{T M}$ sobre $\mathbf{M}$ e $i d_{\mathbf{M}}$ es la aplicación identidad sobre $\mathbf{M}$.

$\mathfrak{X}(\mathbf{M})=\mathcal{J}_{0}^{1}(\mathbf{M})=\{$ campos vectoriales sobre $\mathbf{M}\}$.

$\mathfrak{X}^{*}(\mathbf{M})=\mathcal{J}_{1}^{0}(\mathbf{M})=\{$ covectores o 1 -formas diferenciales sobre $\mathbf{M}\}$.

Dadas dos variedades $\mathbf{M}$ y $\mathbf{N}$ y una función $F: \mathbf{M} \longrightarrow \mathbf{N}$ de clase $C^{\infty}$ se llama aplicación tangente de $F$ en $m$ y se denota por $T_{m} F$ o por $F_{* m}$ a la aplicación lineal

$$
F_{* m}: \mathbf{T}_{\mathbf{m}} \mathbf{M} \longrightarrow \mathbf{T}_{\mathbf{F}(\mathbf{m})} \mathbf{N}
$$

definida por $F_{* m} X_{m}(f)=X_{m}(f \circ F)$ donde $X_{m} \in \mathbf{T}_{\mathbf{m}} \mathbf{M}$ y $f \in C^{\infty}(F(m))$. Además, si $F$ es un difeomorfismo, entonces $F_{* m}: \mathbf{T}_{\mathbf{m}} \mathbf{M} \longrightarrow \mathbf{T}_{\mathbf{F}(\mathbf{m})} \mathbf{N}$ es un isomorfismo de espacios tangentes con inversa $\left(F_{* m}\right)^{-1}=\left(F^{-1}\right)_{* F(m)}$. Y si $H=F \circ G$ es la composición de dos funciones $C^{\infty}, F$ y $G$, entonces $H_{* m}=F_{* G(m)} \circ G_{* m}$ o también $T_{m} H=T_{G(m)} F \circ T_{m} G$.

En las condiciones del punto anterior, se define la aplicación cotangente de $F$ en $m \mathrm{y}$ se denota por $T_{m}^{*} F$ o por $F_{m}^{*}$ a la aplicación lineal $F_{m}^{*}: \mathbf{T}_{\mathbf{F}(\mathbf{m})}^{*} \mathbf{N} \longrightarrow \mathbf{T}_{\mathbf{m}}^{*} \mathbf{M}$ definida por $\left\langle F_{m}^{*} \alpha_{F(m)}, X_{m}\right\rangle=\left\langle\alpha_{F(m)}, F_{* m} X_{m}\right\rangle$ donde $\alpha_{F(m)} \in \mathbf{T}_{\mathbf{F}(\mathbf{m})}^{*} \mathbf{N} \wedge X_{m} \in \mathbf{T}_{\mathbf{m}} \mathbf{M}$.

Si $F: \mathbf{M} \longrightarrow \mathbf{N}$ es un difeomorfismo de variedades $C^{\infty}$, se definen:

- Pull-back de una función $f \in \mathcal{F}(\mathbf{N})$ como la $F^{*} f=f \circ F \in \mathcal{F}(\mathbf{M})$.

- Push-forward de una $g \in \mathcal{F}(\mathbf{M})$ como el elemento de $\mathcal{F}(\mathbf{N})$ definido por $F_{*} g=$ $g \circ F^{-1}$.

Así mismo, para campos vectoriales se definen:

- Push-forward de un campo vectorial de $X$ sobre $\mathbf{M}$, como el campo vectorial sobre $\mathbf{N}$ definido por la expresión $\left(F_{*} X\right)_{n}=F_{* F^{-1}(n)} X_{F^{-1}(n)}, \forall n \in \mathbf{N}$.

- Pull-back de un campo vectorial de $Y$ sobre $\mathbf{N}$, como el campo vectorial sobre $\mathbf{M}$ definido por la expresión $\left(F^{*} Y\right)_{m}=\left(F_{* m}\right)^{-1} Y_{F(m)}, \forall m \in \mathbf{M}$.

- Pull-back de una $k$ - forma $\Omega$ sobre $\mathbf{N}$, a la $k$-forma $F^{*} \Omega$ sobre $\mathbf{M}$ definida por $\left(F^{*} \Omega\right)_{m}\left(z_{1}, z_{2}, \cdots, z_{k}\right)=\Omega_{F(m)}\left(T_{m} F \cdot z_{1}, T_{m} F \cdot z_{2}, \cdots, T_{m} F \cdot z_{k}\right)$.

- Push-forward de una $k$ - forma $\Omega$ sobre $\mathbf{M}$, a la $k$ - forma $F_{*} \Omega$ sobre $\mathbf{N}$ definida por $F_{*} \Omega=\left(F^{-1}\right)^{*} \Omega$.

\subsubsection{Grupos y álgebras de Lie}

\subsubsection{Situándonos dentro de nuestro problema}

Situémonos en una variedad diferenciable $n$-dimensional $\mathbf{M}$, que va a ser nuestro espacio de configuración. En ella describiremos las ecuaciones del movimiento.

Un campo vectorial $X$ en la variedad diferenciable asigna un vector tangente para cada $m \in \mathbf{M}$, que será diferenciable cuando, de manera local, pueda expresarse como

$$
X(m)=\left(X_{1}(m), \ldots, X_{n}(m)\right)^{t}
$$


donde cada componente es una función diferenciable. A partir de ahora, supondremos que $X$ es un campo vectorial diferenciable.

Llamaremos curva integral del campo $X$, a aquella curva $\gamma$ que verifica que

$$
\dot{\gamma}=X(\gamma(t))
$$

Esta relaciones describen las ecuaciones del movimiento asociadas al campo vectorial $X$ en la variedad $\mathbf{M}$. El flujo del campo vectorial sobre el intervalo $t \in(-\epsilon, \epsilon)$ con $\epsilon>0$ es una familia uniparamétrica de aplicaciones

$$
\phi_{t}: \mathbf{M} \longrightarrow \mathbf{M}
$$

que verifica que $\phi_{t}(m)$ es la única curva integral de $X$ que pasa por $m$ cuando $t=0$.

Sea ahora un campo vectorial $X$ en la variedad $\mathbf{M}$, y $f \in C^{\infty}(\mathbf{M})$. Llamamos derivada de Lie de la función $f$ a una nueva función denotada por $\mathcal{L}_{X} f$ expresada por

$$
\mathcal{L}_{X} f(m)=X_{m}(f)
$$

El corchete de Lie de los campos vectoriales $X$ e $Y$ es el campo vectorial $[X, Y]$ definido por

$$
[X, Y](f)=\mathcal{L}_{X}\left(\mathcal{L}_{Y} f\right)-\mathcal{L}_{Y}\left(\mathcal{L}_{X} f\right)
$$

El conjunto de todos los campos vectoriales $\mathfrak{X}(\mathbf{M})$ sobre una variedad $\mathbf{M}$ tiene estructura de espacio vectorial y con esta operación resulta ser un álgebra de Lie (recordemos que un álgebra de Lie es un espacio vectorial $V$ dotado de una forma bilineal antisimétrica $[\Delta, \Delta]$ que verifica la identidad de Jacobi $[[x, y], z]+[[y, z], x]+[[z, x], y]=0$, para todo $x, y, z \in V)$.

Proposición 12 Sea $\mathbf{M}$ una variedad diferenciable, entonces $\mathfrak{X}(\mathbf{M})$ es un álgebra de Lie respecto a la operación corchete de Lie.

\subsubsection{Definiciones y primeras propiedades}

Los espacios de configuración de ciertos sistemas dinámicos hamiltonianos, además de ser variedades diferenciables verifican otras propiedades. Una propiedad muy importante es que el espacio de configuración tenga estructura de grupo.

Llamamos grupo de Lie a una variedad diferenciable $\mathbf{G}$ dotada de una estructura de grupo consistente con su estructura de variedad, es decir, tal que la operación de grupo multiplicación

$$
(g, h) \longrightarrow g h
$$

es de clase $C^{\infty}$ en $\mathbf{G}$.

Ejemplos de grupo de Lie respecto al producto de matrices son: $\mathbf{G L}(n, \mathbf{C}), \mathbf{O}(n), \mathbf{S O}(n)$. Asociadas a cada grupo de Lie existen dos aplicaciones

$$
\begin{aligned}
& L_{g}: \mathbf{G} \longrightarrow \mathbf{G} \\
& h \longrightarrow L_{g}(h)=g h \\
& R_{h}: \mathbf{G} \longrightarrow \mathbf{G} \\
& g \longrightarrow R_{h}(g)=g h
\end{aligned}
$$


denominadas traslaciones por la izquierda y por la derecha respectivamente.

Además, verifican las propiedades siguientes:

$$
\begin{aligned}
L_{g_{1}} \circ L_{g_{2}} & =L_{g_{1} g_{2}} \\
R_{h_{1}} \circ R_{h_{2}} & =R_{h_{2} h_{1}}
\end{aligned}
$$

Si $e \in \mathbf{G}$ es el elemento identidad, es claro que

$$
L_{e}=R_{e}=I d_{\mathbf{G}}
$$

y se verifica que

$$
\begin{gathered}
\left(L_{g}\right)^{-1}=L_{g^{-1}} \\
\left(R_{h}\right)^{-1}=R_{h^{-1}}
\end{gathered}
$$

Así $L_{g}$ y $R_{h}$ son difeomorfismos para cada $g$ y $h$. Además

$$
L_{g} \circ R_{h}=R_{h} \circ L_{g}
$$

Diferenciando $l_{g^{-1}} \circ l_{g}=I d_{\mathbf{G}}$ se obtiene

$$
T_{g h} L_{g^{-1}} \circ T_{h} l_{g}=T_{h}\left(l_{g^{-1}} \circ l_{g}\right)=I d_{T_{h \mathrm{G}}}
$$

Como consecuencia de las relaciones anteriores las aplicaciones $T_{h} L_{g}, T_{g} R_{h}$ son invertibles.

Un campo vectorial $X$ en $\mathbf{G}$ se llama invariante por la izquierda, si para cada $g \in \mathbf{G}$ se tiene que

$$
L_{g}^{*} X=X
$$

es decir, se verifica la fórmula siguiente

$$
\left(T_{h} L_{g}\right) X(h)=X(g h)
$$

con $h \in \mathbf{G}$. Siendo $L_{g}^{*} X$ el pull-back del campo vectorial $X$.

Llamamos $\mathfrak{X}_{L}(\mathbf{G})$ al conjunto de todos los campos vectoriales invariantes por la izquierda en $\mathbf{G}$. Si $g \in \mathbf{G}$, y $X, Y \in \mathfrak{X}_{L}(\mathbf{G})$ entonces

$$
L_{g}^{*}[X, Y]=\left[L_{g}^{*} X, L_{g}^{*} Y\right]=[X, Y]
$$

Se cumple que $\mathfrak{X}_{L}(\mathbf{G})$ es una subálgebra de Lie del álgebra $\mathfrak{X}(\mathbf{G})$ de todos los campos vectoriales sobre $\mathbf{G}$ y se verifica que $\mathfrak{X}_{L}(\mathbf{G})$ y $T_{e} \mathbf{G}$ son espacios vectoriales isomorfos.

Definimos un corchete de Lie en $T_{e} \mathbf{G}$ por la fórmula siguiente

$$
[\xi, \eta]=\left[X_{\xi}, X_{\eta}\right](e)
$$

donde $\xi, \eta \in T_{e} \mathbf{G}$ y $\left[X_{\xi}, X_{\eta}\right]$ es el corchete de Jacobi-Lie de sus campos vectoriales asociados, que cumplen que

$$
\left[X_{\xi}, X_{\eta}\right]=X_{[\xi, \eta]}
$$

El espacio vectorial $T_{e} \mathbf{G}$ es un álgebra de Lie asociada al grupo de Lie $\mathbf{G}$ y es denotada por $\mathbf{g}$.

En los grupos de Lie matriciales es usual definir un producto interior expresado por

$$
\langle A, B\rangle=\operatorname{Traza}\left(A B^{T}\right)
$$

muy importante a la hora de generalizar el concepto de giróstato a grupos de Lie arbitrarios. 
Otro ejemplo es el siguiente. Sea en $\mathbb{R}^{n}$ el conjunto de todas las transformaciones lineales que dejan invariante la cuádrica $x_{1}^{2}+\ldots+x_{n}^{2}=1$. Dicho conjunto tiene estructura de grupo de Lie al que denotaremos por $\mathbf{O}(n)$ que además es compacto. Como espacio vectorial tiene dimensión finita siendo esta igual a $n(n-1) / 2$. Su álgebra de Lie $\mathbf{o}(n)$ es el conjunto de todas las matrices antisimétricas con corchete de Lie dado por el conmutador

$$
[A, B]=A B-B A .
$$

El grupo de Lie $\mathbf{O}(n)$ consta de dos componentes conexas, la componente conexa en la identidad de este, es un grupo de Lie compacto denotado por $\mathbf{S O}(n)$ cuya álgebra de Lie coincide con la de $\mathbf{O}(n)$, es decir so $(n)=\mathbf{o}(n)$. Por su interés en los capítulos siguientes, demos algunos ejemplos de grupos de Lie y sus álgebras de Lie correspondientes.

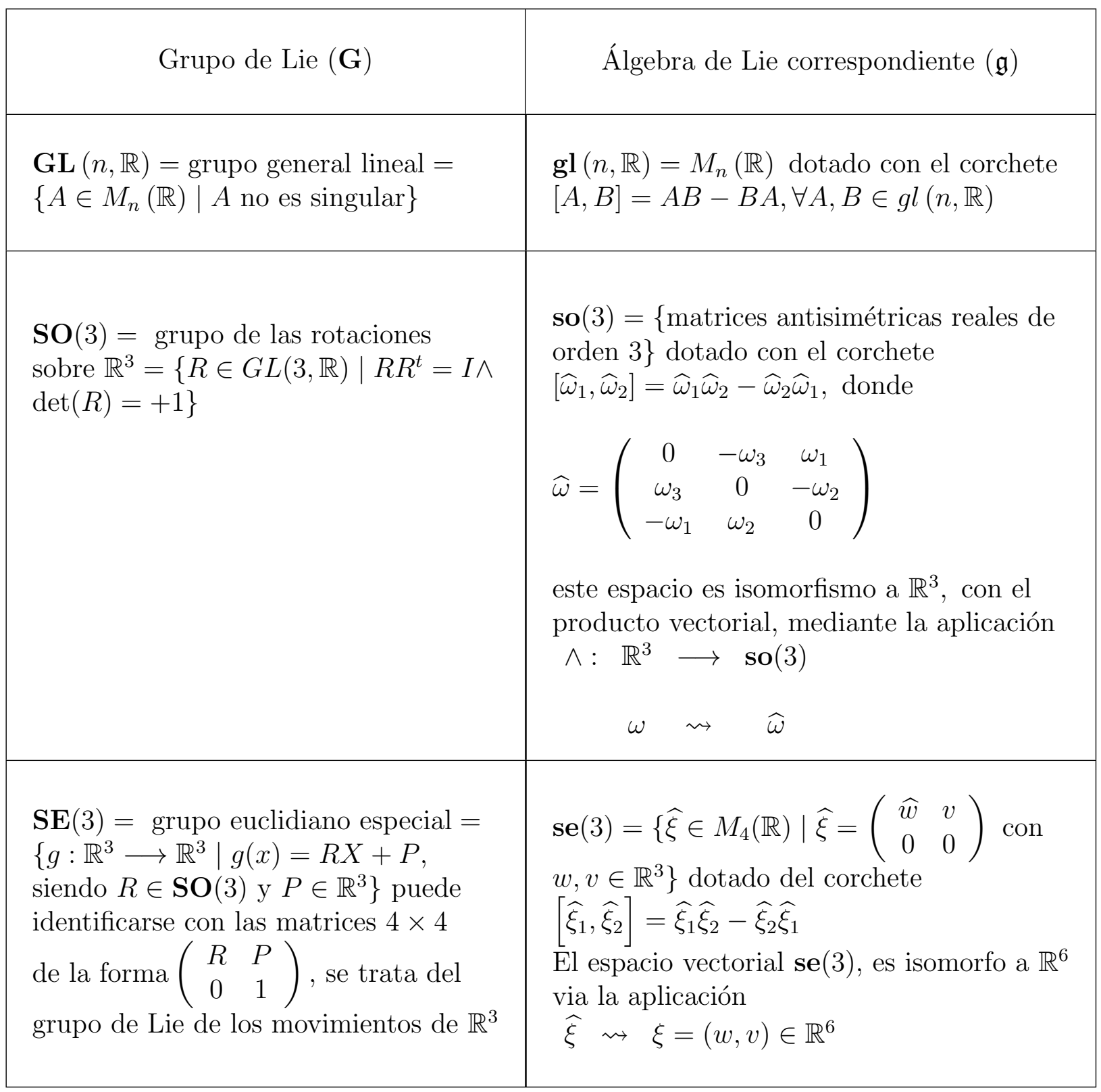

Tabla 2.1: Ejemplos de grupos y álgebras de Lie. 


\subsubsection{Acciones de grupos de Lie}

Sea $\mathbf{M}$ una variedad diferenciable y sea $\mathbf{G}$ un grupo de Lie. Una acción por la izquierda del grupo de Lie $\mathbf{G}$ sobre la variedad $\mathbf{M}$ es una aplicación $C^{\infty}$

$$
\begin{aligned}
\Phi: \mathbf{G} \times \mathbf{M} & \longrightarrow \mathbf{M} \\
(g, m) & \longrightarrow \Phi(g, m)=\Phi_{g}(m)=g m
\end{aligned}
$$

y que verifica las siguientes propiedades:

1. $\Phi(e, m)=m$, para cada $m \in \mathbf{M}$.

2. $\Phi(g, \Phi(h, m))=\Phi(g h, m)$ para cada $g, h \in \mathbf{G}$ y $m \in \mathbf{M}$.

De igual modo, se puede definir una acción por la derecha.

Ahora, si $\mathbf{S O}(3)$ actúa sobre $\mathbb{R}^{3}$

$$
\Phi: \begin{array}{lll}
\mathbf{S O}(3) \times \mathbb{R}^{3} & \longrightarrow & \mathbb{R}^{3} \\
(A, x) & \longrightarrow & \Phi(A, x)=A x
\end{array}
$$

esta acción deja la esfera unidad invariante, luego la misma fórmula define una acción en $S^{2}$. Análogamente $\mathbf{G L}\left(n, \mathbb{R}^{n}\right)$ actua sobre $\mathbb{R}^{n}$ mediante la fórmula anterior.

Sea $\Phi$ una acción de $\mathbf{G}$ en $\mathbf{M}$ y sea $m \in \mathbf{M}$, llamaremos órbita del elemento $m$ al subconjunto

$$
\operatorname{Orb}(m)=\left\{\Phi_{g}(m) / g \in \mathbf{G}\right\}
$$

Para $m \in \mathbf{M}$, llamaremos el grupo de simetrías de la acción $\Phi$ en $m$ al siguiente subconjunto

$$
\mathbf{G} m=\left\{g \in G / \Phi_{g}(m)=m\right\}
$$

Una acción $\Phi$ se dirá:

1. Transitiva cuando solo tenga una órbita, o equivalentemente, si $\forall x, y \in \mathbf{M} \exists g \in G$ tal que $g x=y$.

2. Efectiva si $\Phi_{g}=I d_{\mathbf{M}}$, entonces $g=e$, es decir, la aplicación $g \longrightarrow \Phi_{g}$ es inyectiva.

3. Libre cuando no tenga puntos fijos, es decir, si $\Phi_{g}(x)=x \Longrightarrow g=e$ o equivalentemente si para cada $x \in M, g \longrightarrow \Phi_{g}$ es inyectiva.

4. Propia, si la aplicación

$$
\begin{aligned}
\Psi: \mathbf{G} \times \mathbf{M} & \longrightarrow \mathbf{M} \times \mathbf{M} \\
(g, m) & \longrightarrow
\end{aligned}
$$

verifica que para todo compacto $K \subset \mathbf{M} \times \mathbf{M}, \Psi^{-1}(K)$ es un compacto de $\mathbf{G} \times \mathbf{M}$.

Sea ahora $I_{g}: \mathbf{G} \longrightarrow \mathbf{G}$ el automorfismo interior asociado al elemento $g$. Diferenciando este en la identidad e tenemos la llamada representación adjunta del grupo $\mathbf{G}$ sobre el álgebra $\mathrm{g}$

$$
A d_{g}=T_{e} I_{g}: \mathbf{g} \longrightarrow \mathbf{g}
$$


Explícitamente la acción adjunta, $A d: \mathbf{G} \times \mathbf{g} \longrightarrow \mathbf{g}$ es

$$
A d_{g}(\xi)=T_{e}\left(R_{g^{-1}} \circ L_{g}\right) \cdot \xi
$$

La acción coadjunta de $\mathbf{G}$ sobre $\mathbf{g}^{*}$

$$
\Phi^{*}: \mathbf{G} \times \mathbf{g}^{*} \longrightarrow \mathbf{g}^{*}
$$

donde $\mathbf{g}^{*}$ es el dual del álgebra de Lie $\mathbf{g}$ se apoya en la aplicación $A d_{g}^{*}: \mathbf{g}^{*} \longrightarrow \mathbf{g}^{*}$ viene definida por

$$
\left\langle A d_{g}^{*} \alpha, \xi\right\rangle=\left\langle\alpha, A d_{g} \xi\right\rangle
$$

para $\alpha \in \mathbf{g}^{*}$ y $\xi \in \mathbf{g}$. De esta forma la acción coadjunta de $\mathbf{G}$ sobre $\mathbf{g}^{*}$ tendrá por expresión

$$
\Phi^{*}(g, \alpha)=A d_{g^{-1}}^{*} \alpha
$$

La representación coadjunta de $\mathbf{G}$ sobre $\mathbf{g}^{*}$ es denotada por $A d^{*}: \mathbf{G} \longrightarrow \mathbf{G L}\left(\mathbf{g}^{*}, \mathbf{g}^{*}\right)$ y viene determinada mediante la expresión

$$
A d_{g^{-1}}^{*}=\left(T_{e}\left(R_{g} \circ L_{g^{-1}}\right)\right)^{*}
$$

Una acción $\Phi$ de $\mathbf{G}$ en una variedad $\mathbf{M}$ define una relación de equivalencia en $\mathbf{M}$ como sigue

$$
m_{1} \sim m_{2} \Longleftrightarrow m_{2} \in \operatorname{Orb}\left(m_{1}\right)
$$

con $m_{1}, m_{2} \in \mathbf{M}$.

Sea $\mathbf{M} / \mathbf{G}$ el conjunto de todas las clases de equivalencia, es decir el conjunto de todas las órbitas, entonces considerando la proyección canónica

$$
\begin{array}{cccc}
\pi: & \mathbf{M} & \longrightarrow & \mathbf{M} / \mathbf{G} \\
m & \longrightarrow \pi(m)=\mathbf{O r b}(m)
\end{array}
$$

y dotemos a $\mathbf{M} / \mathbf{G}$ de la topología cociente, tendremos que $\mathbf{M} / \mathbf{G}$ tiene estructura de variedad diferenciable.

Proposición 13 Sea $\Phi: \mathbf{G} \times \mathbf{M} \longrightarrow \mathbf{M}$ una acción propia y libre. Entonces $\mathbf{M} / \mathbf{G}$ es una variedad diferenciable y la aplicación $\pi: \mathbf{M} \longrightarrow \mathbf{M} / \mathbf{G}$ es una inmersión diferenciable.

\subsection{Variedades simplécticas y de Poisson}

Definición 14 Sea $\mathbf{M}$ una variedad y sea $\mathcal{F}(\mathbf{M})$ el conjunto de las funciones reales de clase $C^{\infty}$ sobre $\mathbf{M}$. Un corchete de Poisson $\{\cdot, \cdot\}$ en $\mathbf{M}$ es una aplicación bilineal:

$$
\{\cdot, \cdot\}: \mathcal{F}(\mathbf{M}) \times \mathcal{F}(\mathbf{M}) \longrightarrow \mathcal{F}(\mathbf{M})
$$

satisfaciendo las siguientes propiedades:

1. Anticomutativa: $\{f, g\}=-\{g, f\}$.

2. Identidad de Jacobi: $\{\{f, g\}, h\}+\{\{h, f\}, g\}+\{\{g, h\}, f\}=0$.

3. La regla de Leibniz: $\{f g, h\}=f\{g, h\}+g\{f, h\}$. 
El par $(\mathbf{M},\{\cdot, \cdot\})$ es llamada variedad de Poisson.

La bilinealidad y la regla de Leibniz implican la existencia del tensor $B$ en $\mathbf{M}$ asignando a cada $z \in \mathbf{M}$ una aplicación lineal $B(z): \mathbf{T}_{z}^{*} \mathbf{M} \longrightarrow \mathbf{T}_{z} \mathbf{M}$ tal que

$$
\{f, g\}=\langle B(z) \cdot d f(z), d g(z)\rangle,
$$

donde $\langle\cdot, \cdot\rangle$ denota la relación natural entre vectores y covectores.

Definición 15 Sean $\left(\mathbf{M}_{1},\{\cdot, \cdot\}_{1}\right)$ y $\left(\mathbf{M}_{2},\{\cdot, \cdot\}_{2}\right)$ dos variedades de Poisson. Una aplicación $\psi: \mathbf{M}_{1} \longrightarrow \mathbf{M}_{2}$ es llamada de Poisson si para todo $f, g \in \mathcal{F}\left(\mathbf{M}_{\mathbf{2}}\right)$ tenemos

$$
\{f, g\}_{2} \circ \psi=\{f \circ \psi, g \circ \psi\}_{1}
$$

Definición 16 Sea $\mathbf{M}$ una variedad y $\Omega$ una 2 -forma en $\mathbf{M}$, es decir,

$$
\begin{aligned}
& \Omega: \mathbf{M} \longrightarrow \wedge^{2} \mathbf{M} \\
& m \longrightarrow \Omega(m): \quad \mathbf{T}_{m} \mathbf{M} \times \mathbf{T}_{m} \mathbf{M} \longrightarrow \mathbb{R} \\
& (u, v) \quad \longrightarrow \quad \Omega(m)(u, v)
\end{aligned}
$$

La variedad $\mathbf{M}$ se llama variedad simpléctica si la 2 -forma $\Omega$ es simpléctica, es decir, si verifica las dos propiedades siguientes

- $d \Omega=0$, es decir, $\Omega$ es cerrada.

- $\Omega$ es no degenerada, es decir, $\Omega(m)(u, v)=0 \forall u$ implica $v=0$.

Definición 17 Sea $(\Omega, \mathbf{M})$ una variedad simpléctica y sea $f \in \mathcal{F}(\mathbf{M})$ una función real de clase infinito sobre $\mathbf{M}$. Sea $X_{f}$ el único campo vectorial sobre $\mathbf{M}$ que satisface

$$
\Omega(m)\left(X_{f}(m), v\right)=d f(m) \cdot v, \forall v \in \mathbf{T}_{m} \mathbf{M}
$$

Llamamos a $X_{f}$ el campo vectorial hamiltoniano de $f$. Las ecuaciones de Hamilton son las ecuaciones diferenciales sobre $\mathbf{M}$

$$
\dot{z}=X_{f}(z)
$$

En una variedad simpléctica $(\mathbf{M}, \Omega)$ siempre hay asociado un corchete de Poisson definido como sigue

$$
\{f, g\}=\Omega\left(X_{f}, X_{g}\right), f, g \in \mathcal{F}(\mathbf{M})
$$

Por tanto, toda variedad simpléctica es una variedad de Poisson.

Teorema 18 (De Darboux) Supongamos que $\Omega$ es una 2-forma en una variedad $2 n$ dimensional $\mathbf{M}$. Entonces $d \Omega=0$ si y solo si hay una carta $(U, \psi)$ para cada $m \in \mathbf{M}$ tal que $\psi(m)=0, y$ con $\psi(u)=\left(x_{1}(u), \cdots, x_{n}(u), y_{1}(u), \cdots, y_{n}(u)\right)$ tenemos

$$
\left.\Omega\right|_{U}=\sum_{1=1}^{n} d x_{i} \wedge d y_{i}
$$


Las cartas $(U, \psi)$ dadas por el teorema de Darboux son llamadas cartas simplécticas para M, y las funciones componentes $x_{i}, y_{i}$ son llamadas coordenadas canónicas.

Definición 19 Sean $(\mathbf{M}, \Omega)$ y $(\mathbf{N}, \Psi)$ dos variedades simplécticas. Una aplicación $C^{\infty}$, $F: \mathbf{M} \longrightarrow \mathbf{N}$ es llamada simpléctica si $F^{*} \Psi=\Omega$.

Observación 20 El fibrado tangente a una variedad $\mathbf{T}^{*} \mathbf{M}$ tiene estructura natural de variedad simpléctica con la dos forma

$$
\Omega=\sum_{i=1}^{n} d q_{i} \wedge d P_{i}
$$

Además, see verifica que $\Omega=-d \omega^{1}$, siendo $\omega^{1}$ la 1 -forma canónica sobre $\mathbf{T}^{*} \mathbf{M}$ (o uno forma de Cartan) definida en coordenadas simplécticas por $\omega^{1}=\sum_{i=1}^{n} P_{i} d q_{i}$.

Definición 21 Sea $(\mathbf{M},\{\cdot, \cdot\})$ una variedad de Poisson y sea $f \in \mathcal{F}(\mathbf{M})$. Definimos $X_{f}$ como el único campo vectorial sobre $\mathbf{M}$ que satisface que

$$
X_{f}[k]:=\left\langle d k, X_{f}\right\rangle=\{k, f\}, \forall k \in \mathcal{F}(\mathbf{M}) .
$$

Llamamos a $X_{f}$ el campo vectorial hamiltoniano de $f$.

Dado $\mathcal{H} \in \mathcal{F}(\mathbf{M})$ se llaman ecuaciones de Hamilton correspondientes al campo vectorial hamiltoniano de $\mathcal{H}$ a las dadas por

$$
\dot{G}=\{G, H\}, \forall G \in(\mathbf{M})
$$

Proposición 22 Sea $\varphi_{t}$ un flujo sobre una variedad de Poisson $(\mathbf{M},\{\cdot, \cdot\})$ y sea $\mathcal{H} \in$ $\mathcal{F}(\mathbf{M})$. Entonces:

i) Para cada $F \in \mathcal{F}(U)$, con $U$ abierto de $\mathbf{M}$,

$$
\frac{d}{d t}\left(F \circ \varphi_{t}\right)=\{F, H\} \circ \varphi_{t}=\left\{F \circ \varphi_{t}, H\right\}
$$

si y solo si $\varphi_{t}$ es un flujo de $X_{H}$.

ii) Si $\varphi_{t}$ es un flujo de $X_{H}$, entonces $H \circ \varphi_{t}=H$.

Corolario 23 Sean $G, H \in \mathcal{F}(\mathbf{M})$. Entonces $G$ es constante a lo largo de las curvas integrales de $X_{H}$ si y solo si $\{G, H\}=0$. Esta afirmación es equivalente a decir que $H$ es constante a lo largo de las curvas integrales de $X_{G}$.

Definición 24 Se dice que $C \in \mathcal{F}(\mathbf{M})$ es una función de Casimir si $\{C, F\}=0$ para toda $F \in \mathcal{F}(\mathbf{M})$, tal que $C$ es constante a lo largo del flujo de todos los campos vectoriales hamiltonianos. 
Además de las variedades simplécticas, también es posible dotar de estructura de variedad de Poisson a las $\mathbf{g}^{*}$, mediante los corchetes de Lie-Poisson definidos por

$$
\begin{aligned}
& \{F, G\}_{+}(\mu)=\left\langle\mu,\left[\frac{\partial F}{\partial \mu}, \frac{\partial G}{\partial \mu}\right]\right\rangle \\
& \{F, G\}_{-}(\mu)=-\left\langle\mu,\left[\frac{\partial F}{\partial \mu}, \frac{\partial G}{\partial \mu}\right]\right\rangle
\end{aligned}
$$

con $\mu \in \mathbf{g}^{*}$ y $F, G \in \mathfrak{F}\left(\mathbf{g}^{*}\right)$.

Podemos definir el corchete de Frozen-Lie-Poisson si fijamos un elemento $\mu_{0} \in \mathbf{g}^{*} \mathrm{y}$ $F, G \in \mathfrak{F}\left(\mathbf{g}^{*}\right)$

$$
\{F, G\}_{ \pm}(\mu)= \pm\left\langle\mu_{0},\left[\frac{\partial F}{\partial \mu}, \frac{\partial G}{\partial \mu}\right]\right\rangle
$$

Este corchete se utiliza mucho a la hora de describir las ecuaciones de Lie-Poisson en un entorno de un punto de equilibrio.

Por último, definimos el siguiente corchete de Poisson que será muy importante para generalizar la noción de giróstato a un álgebra de Lie cualquiera

$$
\{F, G\}_{ \pm}(\mu)= \pm\left\langle a \mu+\mu_{0},\left[\frac{\partial F}{\partial \mu}, \frac{\partial G}{\partial \mu}\right]\right\rangle
$$

$\operatorname{con} \mu, \mu_{0} \in \mathbf{g}^{*}$ y $F, G \in \mathfrak{F}\left(\mathbf{g}^{*}\right)$ y $a \in \mathbb{R}$.

\subsection{Aplicaciones momento y teoremas de reducción}

Las aplicaciones momento son una generalización geométrica de los clásicos momentos lineal y angular de un sistema material de puntos, y sirven para obtener cantidades conservadas en sistemas lagrangianos o hamiltonianos con simetrías. Aquí nos restringiremos a estos últimos ya sea para el caso de hamiltonianos sobre variedades de Poisson o simplécticas.

\subsubsection{Aplicaciones momento}

Definición 25 Para cada $\xi \in T_{e} \mathbf{G}$, denotaremos por $\Phi_{\xi}: \mathbb{R} \longrightarrow \mathbf{G}$ la curva integral del campo vectorial invariante a izquierda $X_{\xi}$ pasando por e en $t=0$; esto es

$$
\Phi_{\xi}(0)=e \quad y \quad \frac{d}{d t} \Phi_{\xi}(t)=X_{\xi}\left(\Phi_{\xi}(t)\right)
$$

La función exponencial $\exp : \mathfrak{g}=T_{e} \mathbf{G} \longrightarrow \mathbf{G}$ se define por $\exp (\xi)=\Phi_{\xi}(1)$ y verifica $q u e \exp (\xi s)=\Phi_{\xi}(s)$.

Definición 26 Supongamos que $\Phi: \mathbf{G} \times \mathbf{M} \rightarrow \mathbf{M}$ es una acción $C^{\infty}$. Si $\xi \in T_{e} G$, entonces

$$
\begin{aligned}
& \Phi^{\xi}: \mathbb{R} \times \mathbf{M} \longrightarrow \mathbf{M} \\
& (t, x) \longmapsto \Phi(\exp t \xi, x)
\end{aligned}
$$


es una $\mathbb{R}$-acción sobre $\mathbf{M}$, es decir, $\Phi^{\xi}$ es un flujo sobre $\mathbf{M}$. El correspondiente campo vectorial sobre $M$, viene dado por

$$
\xi_{M}=\left.\frac{d}{d t} \Phi(\exp t \xi, x)\right|_{t=0}
$$

y es llamado el generador infinitesismal de la acción correspondiente a $\xi$.

Veamos ahora un importante tipo de integrales en sistemas hamiltonianos.

Definición 27 Sea $(\mathbf{M}, \Omega)$ una variedad simpléctica (o de Poisson) conexa y $\Phi: \mathbf{G} \times$ $\mathbf{M} \rightarrow \mathbf{M}$ una acción simpléctica (o de Poisson) del grupo de Lie $\mathbf{G}$ en $\mathbf{M}$. Se dice que una aplicación

$$
J: \mathbf{M} \longrightarrow \mathfrak{g}^{*} \text { (donde } \mathfrak{g}^{*} \text { es el dual del álgebra de Lie de } G \text { ) }
$$

es una aplicación momento para la acción dada para cada $\xi \in \mathfrak{g}$,

$$
X_{\widehat{J}(\xi)}=\xi_{\mathbf{M}}
$$

donde $\widehat{J}(\xi): \mathbf{M} \longrightarrow \mathbb{R}$ está definida por $\widehat{J}(\xi)(x)=\langle J(x), \xi\rangle$ y $\xi_{\mathbf{M}}$ es el generador infinitesimal de la acción correspondiente a $\xi$.

Saquemos ahora a colación la importancia de las aplicaciones momento con el siguiente teorema:

Teorema 28 (De Noether) Sea $\Phi$ una acción simpléctica (o de Poisson) de G sobre una variedad simpléctica (o de Poisson) $\mathbf{M}$ con una aplicación momento J. Supongamos que $H: \mathbf{M} \longrightarrow \mathbb{R}$ es invariante bajo la acción, esto es,

$$
H(x)=H\left(\Phi_{g}(x)\right), \text { para todo } x \in \mathbf{M}, g \in \mathbf{G} \text {. }
$$

Entonces, $J$ es una integral para $X_{H}$, es decir, si $\varphi_{t}$ es un flujo de $X_{H}$,

$$
J\left(\varphi_{t}(x)\right)=J(x)
$$

Finalmente, decimos que una aplicación momento $J$ es $A d^{*}$-equivariante si

$$
J\left(\Phi_{g}(x)\right)=A d_{g^{-1}}^{*} J(x)
$$

para cada $g \in \mathbf{G}$, es decir, el siguiente diagrama conmuta:

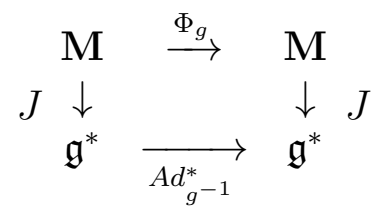




\subsubsection{Teoremas de reducción}

Comencemos hablando de la reducción de Poisson. Sea $\mathbf{M}$ una variedad de Poisson y sea $\mathbf{G}$ un grupo de Lie actuando sobre $\mathbf{M}$. Supongamos que $\mathbf{M} / \mathbf{G}$ es una variedad diferenciable. En este caso la estructura de la variedad de Poisson para $\mathbf{M} / \mathbf{G}$ será la única que hace que la proyección canónica $\pi: \mathbf{M} \longrightarrow \mathbf{M} / \mathbf{G}$ sea una aplicación de Poisson. El siguiente teorema muestra cómo obtener esta situación.

Teorema 29 Sea $(\mathbf{M},\{\cdot, \cdot\})$, una variedad de Poisson y $\mathbf{G}$ un grupo de Lie que nos da la acción $\Phi$ sobre $\mathbf{M}$ tal que la acción es de Poisson. Supongamos que $\Phi$ es libre y propia. Entonces, la variedad cociente $\mathbf{M} / \mathbf{G}$ lleva una estructura de Poisson dada por el corchete de Poisson $\{\cdot, \cdot\}_{\mathbf{M} / \mathbf{G}}$ definido por

$$
\{f, g\}_{\mathbf{M} / \mathbf{G}} \circ \pi=\{f \circ \pi, g \circ \pi\}
$$

para todas $f, g \in \mathcal{F}(\mathbf{M} / \mathbf{G})$, donde $\pi: \mathbf{M} \longrightarrow \mathbf{M} / \mathbf{G}$ es la proyección canónica. Además, la proyección canónica $\pi$ es una aplicación de Poisson para esta estructura de Poisson.

Consideremos ahora que tenemos una función hamiltoniana $H \in \mathcal{F}(\mathbf{M})$. Supongamos que $H$ es $G$-invariante, es decir,

$$
H\left(\Phi_{g}(x)\right)=H(x) \text {, para todo } x \in \mathbf{M}, g \in \mathbf{G} \text {. }
$$

Entonces, la función hamiltoniana $\widetilde{H} \in \mathcal{F}(\mathbf{M} / \mathbf{G})$ está dada por

$$
\widetilde{H}(z)=H(x), x \text { es un representante de clase de } z \text { que está bien definida. }
$$

Y el campo vectorial hamiltoniano $X_{\widetilde{H}}$ está defindo por

$$
X_{\widetilde{H}}[f]=\{f, \widetilde{H}\}_{\mathbf{M} / \mathbf{G}}
$$

y verifica

$$
X_{\widetilde{H}}[f] \circ \pi=\{f \circ \pi, H \circ \pi\} .
$$

Si $\varphi_{t}$ denota el flujo del sistema de Hamilton-Poisson $(\mathbf{M},\{\cdot, \cdot\}, H)$, y $\widetilde{\varphi}_{t}$ denota el flujo del sistema de Hamilton-Poisson $\left(\mathbf{M} / \mathbf{G},\{\cdot, \cdot\}_{\mathbf{M} / \mathbf{G}}, \widetilde{H}\right)$, tenemos que

$$
\pi \circ \varphi_{t}=\widetilde{\varphi}_{t} \circ \pi
$$

Se dice que el sistema Hamilton-Poisson $(\mathbf{M},\{\cdot, \cdot\}, H)$ se reduce al sistema reducido de Hamilton-Poisson $\left(\mathbf{M} / \mathbf{G},\{\cdot, \cdot\}_{\mathbf{M} / \mathbf{G}}, \widetilde{H}\right)$.

Seguidamente establecemos el procedimiento de reducción para una variedad simpléctica. Sea $(\mathbf{M}, \Omega)$ una variedad simpléctica y sea $\Phi: \mathbf{G} \times \mathbf{M} \rightarrow \mathbf{M}$ una acción simpléctica. Supongamos que existe una aplicación momento $A d^{*}$-equivariante $J: \mathbf{M} \longrightarrow \mathfrak{g}^{*}$. Denotamos por $G_{\mu}$ el grupo de isotropía de $\mathbf{G}$ bajo la acción coadjunta $A d^{*}$, es decir,

$$
G_{\mu}=\left\{g \in \mathbf{G}: A d_{g^{-1}}^{*} \mu=\mu\right\} .
$$

Luego, el espacio de órbitas $\mathbf{M}_{\mu}:=\mathbf{J}^{\mathbf{- 1}}(\mu) / \mathbf{G}_{\mu}$ está bien definido. Llamamos a este espacio el espacio fásico reducido. 
Impongamos ahora las condiciones para obtener que $\mathbf{M}_{\mu}$ sea una variedad. Para ello primero asumimos que $\mu$ es un valor regular para la aplicación $J$. Entonces, $\mathbf{J}^{\mathbf{- 1}}(\mu)$ es una subvariedad de $\mathbf{M}$. En segundo lugar, supongamos que $G_{\mu}$ actúa libremente y propiamente sobre $\mathbf{J}^{-\mathbf{1}}(\mu)$. Por tanto, $\mathbf{J}^{-\mathbf{1}}(\mu) / \mathbf{G}_{\mu}$ es una variedad y la proyección canónica $\pi_{\mu}: \mathbf{J}^{-\mathbf{1}}(\mu) \longrightarrow \mathbf{M}_{\mu}$ es una inmersión diferenciable. En definitiva, tenemos el siguiente teorema:

Teorema 30 Sea $(\mathbf{M}, \Omega)$ una variedad simpléctica y $\mathbf{G}$ un grupo de Lie que actúa sobre M simplécticamente. Supongamos que existe una aplicación momento Ad*-equivariante $J$ para esta acción. Asumamos que $\mu \in \mathfrak{g}^{*}$ es un valor regular para $J$ y que el grupo de isotropía $G_{\mu}$ bajo la acción Ad* sobre $\mathfrak{g}^{*}$ actúa libremente y propiamente sobre $\mathbf{J}^{-\mathbf{1}}(\mu)$. Entonces $\mathbf{M}_{\mu}=\mathbf{J}^{-\mathbf{1}}(\mu) / \mathbf{G}_{\mu}$ tiene una única forma simpléctica $\Omega_{\mu}$ con la propiedad

$$
\pi_{\mu}^{*} \Omega_{\mu}=i_{\mu}^{*} \Omega
$$

donde $\pi_{\mu}: \mathbf{J}^{-\mathbf{1}}(\mu) \longrightarrow \mathbf{M}_{\mu}$ es la proyección canónica e $i_{\mu}: \mathbf{J}^{-\mathbf{1}}(\mu) \longrightarrow \mathbf{M}$ es la inclusión.

Por último, inducimos sistemas hamiltonianos sobre los espacios reducidos. El procedimiento es resumido en el siguiente teorema.

Teorema 31 Asumamos todas las hipótesis del teorema anterior y sea $H \in \mathcal{F}(\mathbf{M})$ una función hamiltoniana $G$-invariante. Entonces el flujo $\varphi_{t}$ de $X_{H}$ deja $\mathbf{J}^{-\mathbf{1}}(\mu)$ invariante y conmuta con la acciónde $G_{\mu}$ sobre $\mathbf{J}^{-\mathbf{1}}(\mu)$, así induce canónicamente un flujo $\widetilde{\varphi}_{t}$ sobre $\mathbf{M}_{\mu}$ satisfaciendo $\pi_{\mu} \circ \varphi_{t}=\widetilde{\varphi}_{t} \circ \pi_{\mu}$. Esta flujo es un flujo hamiltoniano sobre $\mathbf{M}_{\mu}$ con un hamiltoniano $H_{\mu}$ satisfaciendo $H_{\mu} \circ \pi_{\mu}=H \circ i_{\mu}$. $H_{\mu}$ es llamado el hamiltoniano reducido. 



\section{3}

\section{Dinámica hamiltoniana de un giróstato en el problema de $n$ cuerpos}

\subsection{Introducción}

Toda la teoría cualitativa moderna de ecuaciones diferenciales ordinarias parte del trabajo de Poincaré, para tratar de explicar el que es el problema fundamental de la mecánica celeste

\section{¿ES EL SISTEMA SOLAR ESTABLE?}

Es decir, si los planetas seguirán en sus órbitas o algunos de ellos, afectados por la proximidad de otros, escaparán de sus órbitas para siempre.

V.I.Arnold (1963) [5], en su artículo "Small Denominators, II: Proof of a Theorem by A.N.Kolmogorov on the Preservation of Conditionals Periodic Motion under a Small Perturbation of the Hamiltonian", realizó una pequeña demostración de que el sistema solar es estable bajo hipótesis adicionales. De esta pregunta surge un desarrollo matemático que va desde el problema de los dos cuerpos hasta el problema de $n$ cuerpos.

Se considera el problema de los dos cuerpos clásico que consiste en determinar el movimiento de dos cuerpos que interactúan gravitatoriamente entre sí. Algunos ejemplos son: la Luna orbitando la Tierra y en ausencia del Sol, es decir aislados; un planeta orbitando una estrella; dos estrellas que giran en torno al centro de masas (estrella binaria), y un electrón orbitando en torno a un núcleo atómico.

Newton resolvió geométricamente este problema considerando los dos cuerpos como masas puntuales. Algunas generalizaciones más realistas del problema surgen al considerar:

- Un sólido y una masa puntual.

- Dos sólidos.

En estos casos aparecen sistemas de ecuaciones diferenciales ordinarias fuertemente no lineales. En general, este tipo de problemas, sólo pueden ser abordados mediante métodos numéricos, pero bajo hipótesis adicionales realistas pueden ser llevados a cabo estudios 
para describir cualitativamente dichas ecuaciones o efectutar interacciones analíticas mediante la teoría de perturbaciones.

El matemático francés Henri Poincaré (1854-1912)

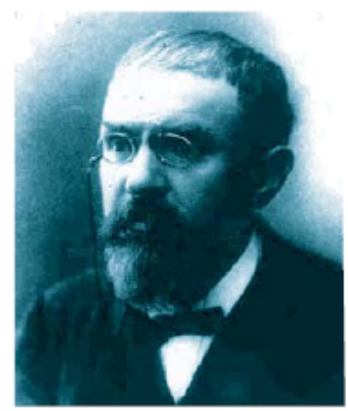

H. Poincaré

a los 26 años escribió una memoria sobre el problema de los tres cuerpos que resultó ganadora de la competición internacional para conmemorar el 60 aniversario de Oscar II, rey de Suecia y Noruega. El objeto del concurso organizado por el rey Oscar II era responder a la pregunta de si el sistema solar es estable.

El problema de los tres cuerpos se puede enunciar de un modo muy sencillo: sean tres partículas de masas diferentes que se mueven en el espacio bajo la acción de la gravitación mutua; dadas unas condiciones iniciales, determínese el movimiento de cada una de las partículas.

Aunque el problema tiene un enunciado de gran simplicidad, su solución no es sencilla en absoluto, Poincaré demostró que para este problema, en general, no era posible encontrar una solución exacta tal y como la conocemos para el problema de los dos cuerpos.

Para el problema de los $n$ cuerpos el estudio es muy complicado, de ahí la complejidad de responder a nuestra pregunta de si el sistema solar es o no estable. Este es un tema de investigación pasado, presente y futuro.

Antes de hacer un pequeño repaso por algunos de los numerosos autores que han estudiado el problema de los 2, 3 y $n$ cuerpos cuando alguno o varios son sólidos rígidos o giróstato. Recordemos el concepto de giróstato, que será considerado a lo largo de todo este trabajo.

Definición 32 Un giróstato es un sistema mecánico $S_{0}$, constituido por un sólido rígido $S^{\prime}$ al que hay conectados otros cuerpos $S^{\prime \prime}$, variables o rígidos pero no rígidamente unidos a $S^{\prime}$, de tal manera que los movimientos de $S^{\prime \prime}$ relativos a $S^{\prime}$ no modifican la distribución de masas del sistema compuesto $S_{0}$, véase Leimanis (1965) [34]. 


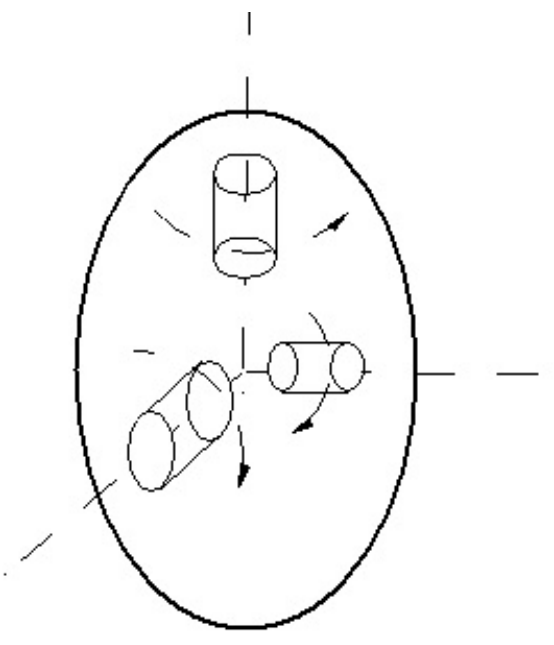

Figura 3.1: Giróstato con tres rotores

El modelo que representa el giróstato, esto es, un cuerpo rotante con cierto momento angular interno, ha sido utilizado con éxito en el estudio de la dinámica de diferentes sistemas físicos desde finales del siglo XIX. Así Peano y Volterra aplicaron el modelo del giróstato a la rotación de la Tierra para explicar el movimiento de los polos terrestres y la variación de la latitud en la superficie de la Tierra por medio de movimientos internos que no modifican su distribución de masas.

En las últimas décadas el estudio de la dinámica de los giróstatos ha adquirido un gran interés en astrodinámica e ingeniería aeroespacial. Esto es debido a que en la actualidad, la mayoría de los satélites artificiales posee uno o más discos giratorios para conseguir el control y la estabilizacón del vehículo de tal manera que este mantenga una cierta orientación deseada.

Recientemente numerosos autores han estudiado distintos problemas sobre giróstatos en diferentes situaciones y la mayoría en relación a la dinámica de satélites artificiales o al estudio de la rotación terrestre, véase por ejemplo, Elipe (1997) [24], Wang et al. (1995) [67] y Vera y Vigueras (2005) [61] y las referencias que encontramos en ellas.

Volvamos al problema de 2, 3 y $n$ cuerpos. Numerosos autores han estudiado el problema de los dos cuerpos. Resaltemos Wang et al. (1991) [66] que estudia el caso de un sólido en un campo central Newtoniano, donde la dinámica no canónica hamiltoniana se obtiene por medio de la única reducción de Poisson. Wang et al. (1992), que [66] desde un punto de vista lagrangiano, construye una teoría sistemática de sistemas girostáticos con simetrías. Una visión más general del problema, es tratada en Barkin et al. (1982) [13] y la dinámica hamiltoniana no canónica del problema de dos sólidos es estudiada en Maciejewski (1995) [37]. Todos estos artículos se generalizan al caso de un giróstato en un campo central newtoniano por Wang et al. (1995) [67] y Mondéjar y Vigueras (1999) [47] cuando estamos en el caso de dos giróstatos en interacción newtoniana.

Respecto al problema de los tres cuerpos rígidos, Vidiakin (1977) [63] y Duboshin (1984) [22] prueban la existencia de equilibrios de Euler y Lagrange cuando los cuerpos poseen simetrías. Zhuravlev y Petrutskii (1990) [68] hacen una revisión de los resultados anteriores. Fanny Cosson y Elmabsout (1997) [26] estudian la configuración de los equilibrios en términos de las variables globales del problema sin reducir, donde los dos cuerpos tienen una distribución de masas esférica y el tercero de ellos es un sólido. Y Mondéjar 
et al. (2001) [48] consideran el problema de los tres cuerpos en el caso en que dos de ellos tienen una distribución de masas esférica y el tercero de ellos es un giróstato.

Por último, en Vera y Vigueras ((2004) [60], (2005) [61], (2006) [62]), se hace un estudio general del problema de los $n$ cuerpos, donde dichos cuerpos son sólidos rígidos o giróstatos, cuyos momentos girostáticos son constantes. Concretamente, se considera la dinámica no canónica del hamiltoniano de $n+1$ cuerpos en atracción newtoniana, donde $n$ de ellos son sólidos rígidos con una distribución esférica de masas (o masas puntuales) y el otro es un giróstato triaxial cuyo momento girostático es constante.

Repasemos algunos de los resultados generales, obtenidos en este último artículo que nos servirán para entender mejor todo lo hecho en este trabajo.

\subsection{Espacios de configuración y fásico}

En lo que sigue utilizaremos la siguiente notación. Sea $S_{0}$ es un giróstato de masa $m_{0}$ y $S_{1}, S_{2}, \ldots, S_{n}, n$ cuerpos rígidos esféricos (o masas puntuales) de masa $m_{1}, m_{2}, \cdots, m_{n}$ respectivamente. $\mathcal{I}=\left\{\mathbf{O}, \mathbf{u}_{1}, \mathbf{u}_{2}, \mathbf{u}_{3}\right\}$ un sistema de referencia inercial y $\mathfrak{J}=\left\{\mathbf{C}_{0}, \mathbf{b}_{1}, \mathbf{b}_{2}, \mathbf{b}_{3}\right\}$ un sistema de referencia ligado al cuerpo con centro en el centro de masas $\mathbf{C}_{0}$ de $S_{0}$. Por último definimos por $R_{i}$ los vectores de posición respecto al centro de masas de $S_{i}$ en $\mathcal{I}$.

Por lo tanto, una partícula de $S_{0}$ con coordenadas $Q$ en $\mathfrak{J}$ es representada en el sistema inercial $\mathcal{I}$ por el vector $\mathbf{q}=\mathbf{R}_{0}+B \mathbf{Q}$ con $B \in \mathbf{S O}(3)$.

Así pues, el espacio de configuración del problema es el grupo de Lie

$$
\mathbf{Q}=\mathbf{S E}(3) \times \mathbb{R}^{3} \times \cdots{ }^{n)} \times \mathbb{R}^{3}
$$

siendo SE (3) el producto semidirecto de $\mathbf{S O}(3)$ y $\mathbb{R}^{3}$, cuyos elementos son

$$
\left(\left(B, \mathbf{R}_{0}\right), \mathbf{R}_{1}, \cdots, \mathbf{R}_{n}\right)
$$

El vector $\widehat{\mathbf{v}}$ es la imagen del vector $\mathbf{v} \in \mathbb{R}^{3}$ por el isomorfismo estándar entre las álgebras de Lie $\mathbb{R}^{3}$ y $\mathfrak{s o}(3)$, es decir:

$$
\widehat{\mathbf{v}}=\left(\begin{array}{ccc}
0 & -v_{3} & v_{2} \\
v_{3} & 0 & -v_{1} \\
-v_{2} & v_{1} & 0
\end{array}\right)
$$

Así, si tenemos $\mathbf{u}, \mathbf{v}$ vectores de $\mathbb{R}^{3}, \mathbf{u} \cdot \mathbf{v}$ representa el producto escalar usual, $|\mathbf{u}|$ es la norma euclídea del vector $\mathbf{u}$ y $\mathbf{u} \times \mathbf{v}$ es el producto vectorial.

Tenemos entonces (véase Vera (2004) [59] y Vera y Vigueras (2006) [62] para más detalles), que la energía cinética del sistema es

$$
T=\frac{1}{2} \int_{S_{0}}|\dot{\mathbf{q}}|^{2} d m(\mathbf{Q})+\frac{1}{2} \sum_{i=1}^{n} m_{i}\left|\dot{\mathbf{R}}_{i}\right|^{2}
$$

Esta expresión de la energía cinética, se simplifica a

$$
T=\frac{1}{2} \boldsymbol{\Omega} \cdot \mathbb{I} \boldsymbol{\Omega}+\mathbf{l}_{r} \cdot \boldsymbol{\Omega}+\frac{1}{2} \sum_{i=1}^{n} m_{i}\left|\dot{\mathbf{R}}_{i}\right|^{2}+\mathcal{T}_{r}
$$

Donde $\mathbb{I}=\operatorname{diag}(A, B, C)$, matriz diagonal de orden tres, es el tensor diagonal de inercia asociado al giróstato $S_{0}, \boldsymbol{\Omega}$ es el vector velocidad angular de $S_{0}$ definido por $\dot{B}^{=} B \widehat{\Omega}$ y $\mathbf{l}_{r}$ 
es el momento girostático. Por último, $\mathcal{T}_{r}$ es la energía cinética relativa a el movimiento relativo de $S^{\prime \prime}$ con respecto a $S^{\prime}$. Tanto $\mathbf{l}$ como $\mathcal{T}_{r}$ están originados por el movimiento de la parte móvil del giróstato con respecto a su parte rígida. Se asumirá que $\mathcal{T}_{r}$ es una función conocida del tiempo y que $\mathbf{l}_{r}$ es un vector constante.

La energía potencial del problema será la función $V: \mathbf{Q} \longrightarrow \mathbb{R}$ de la forma

$$
V=-\left(\frac{1}{2} \sum_{\substack{i, j=1 \\ i \neq j}}^{n} \frac{G m_{i} m_{j}}{\left|\mathbf{R}_{i}-\mathbf{R}_{j}\right|}+\sum_{i=1}^{n} \int_{S_{0}} \frac{G m_{i} d m(\mathbf{Q})}{\left|B \mathbf{Q}+\mathbf{R}_{0}-\mathbf{R}_{i}\right|}\right)
$$

Una vez definido el espacio de configuración, pasemos a definir el espacio de fases. Para ello definimos el Langragiano del problema $\mathcal{L}:$ TQ $\longrightarrow \mathbb{R}$

$$
\mathcal{L}=T-V \circ \tau
$$

siendo $\tau: \mathbf{T Q} \longrightarrow \mathbf{Q}$ la proyección canónica. Por otro lado, el espacio de fases $\mathbf{T}^{*} \mathbf{Q}$, está formado por elementos de la forma

$$
\Xi=\left(\left(B, \mathbf{R}_{0}\right), \mathbf{R}_{1}, \cdots, \mathbf{R}_{n},\left(B \widehat{\mathbf{\Pi}}, \mathbf{P}_{0}\right), \mathbf{P}_{1}, \cdots, \mathbf{P}_{n}\right)
$$

donde $\boldsymbol{\Pi}=\mathbb{I} \boldsymbol{\Omega}+\mathbf{l}_{r}$ el vector momento angular total del giróstato en el sistema de referencia ligado al cuerpo $\mathfrak{J}$, y $\mathbf{P}_{i}=m_{i} \dot{\mathbf{R}}_{i}$ son los momentos lineales de los cuerpos en el sistema fijo $\mathcal{I}$. El espacio fásico $\mathbf{T}^{*} \mathbf{Q}$ lleva consigo una estructura canónica simpléctica definida por

$$
\omega=\omega^{\mathbf{S E}(3)}+\omega^{\mathbb{R}^{3}}+\cdots+\omega^{\mathbb{R}^{3}}
$$

Por último asociado a $\omega$, tenemos la estructura de Poisson, donde el corchete de Poisson a tener en cuenta es

$$
\begin{aligned}
\{f, g\}_{\mathbf{T}^{*} \mathbf{Q}}(\Xi) & = \\
& \left(\left\langle D_{B} f, \frac{\partial g}{\partial B \widehat{\boldsymbol{\Pi}}}\right\rangle-\left\langle D_{B} g, \frac{\partial f}{\partial B \widehat{\boldsymbol{\Pi}}}\right\rangle+\sum_{i=1}^{n}\left(\frac{\partial f}{\partial \mathbf{R}_{i}} \frac{\partial g}{\partial \mathbf{P}_{i}}-\frac{\partial g}{\partial \mathbf{R}_{i}} \frac{\partial f}{\partial \mathbf{P}_{i}}\right)\right)(\mathfrak{g})
\end{aligned}
$$

con $<\cdot, \cdot>$ : T*SO $(3) \times \mathbf{T S O}(3) \longrightarrow \mathbb{R}$, tal que

$$
\left\langle\alpha_{A}, \omega_{A}\right\rangle=\frac{1}{2} \operatorname{traza}\left(\alpha_{A}^{T} \omega_{A}\right) .
$$

Por medio de la transformación de Legende obtenemos nuestro hamiltoniano $\mathcal{H}$ : $\mathbf{T}^{*} \mathbf{Q} \longrightarrow \mathbb{R}$ dado por

$$
\mathcal{H}(\mathbf{z})=\frac{1}{2} \boldsymbol{\Pi} \mathbb{I}^{-1} \boldsymbol{\Pi}-\mathbf{l}_{r} \cdot \mathbb{I}^{-1} \boldsymbol{\Pi}+\sum_{i=0}^{n} \frac{\left|\mathbf{P}_{i}\right|^{2}}{2 m_{i}}+V \circ \tau_{\mathbf{T}^{*} \mathbf{Q}}
$$

\subsection{Simetrías y reducción}

Usando la reducción mediante el producto semidirecto (véase Marsden et al. 1984a, b, 1992 [44]), el problema puede reducirse por medio de la acción del grupo de Lie SE(3). Siguiendo la línea de Vera y Vigueras (2004) [60], se procederá por fases, generalizando el trabajo de Mondéjar et al. (2001) [48] y siguiendo la línea de Vera (2004) [59], en un primer paso se procederá mediante una reducción simpléctica debido a la acción del grupo de tanslaciones en $\mathbb{R}^{3}$. En una segunda fase, se procederá a una reducción de Poisson mediante la acción del grupo de Lie $\mathbf{S O}(3)$. 


\subsubsection{Reducción por el grupo de traslaciones}

Sea la acción del grupo translación de $\mathbb{R}^{3}$ en $\mathbf{Q}$

$$
\Phi_{1}: \mathbb{R}^{3} \times \mathbf{Q} \rightarrow \mathbf{Q}
$$

dada por $(\mathbf{u}, \boldsymbol{\Delta}) \in \mathbb{R}^{3} \times \mathbf{Q}$ donde

$$
\Phi_{1}(\mathbf{u}, \boldsymbol{\Delta})=\left(\left(B, \mathbf{R}_{0}+\mathbf{u}\right), \mathbf{R}_{1}+\mathbf{u}, \ldots, \mathbf{R}_{i}+\mathbf{u}, \ldots, \mathbf{R}_{n}+\mathbf{u}\right)
$$

Esta acción se eleva a una acción libre y propia en $\mathbf{T}^{*} \mathbf{Q}$

$$
\Phi_{1}^{\mathbf{T}^{*} \mathbf{Q}}: \mathbb{R}^{3} \times \mathbf{T}^{*} \mathbf{Q} \rightarrow \mathbf{T}^{*} \mathbf{Q}
$$

definida para $(\mathbf{u}, \boldsymbol{\Xi}) \in \mathbb{R}^{3} \times \mathbf{T}^{*} \mathbf{Q}$ por medio de la fórmula

$$
\begin{gathered}
\Phi_{1}^{\mathbf{T}^{*} \mathbf{Q}}(\mathbf{u}, \boldsymbol{\Xi})=\left(\left(B, \mathbf{R}_{0}+\mathbf{u}\right), \mathbf{R}_{1}+\mathbf{u}, \ldots, \mathbf{R}_{i}+\mathbf{u}, \ldots, \mathbf{R}_{n}+\mathbf{u}\right. \\
\left.(B, \widehat{\Pi}), \mathbf{P}_{1}, \ldots, \mathbf{P}_{i}, \ldots, \mathbf{P}_{n}\right)
\end{gathered}
$$

que tienen asociada la aplicación momento

$$
j: \mathbf{T}^{*} \mathbf{Q} \rightarrow \mathbb{R}^{3}
$$

definida mediante la expresión

$$
j(\boldsymbol{\Xi})=\sum_{i=0}^{n} \mathbf{P}_{i}
$$

Si $k$ es un valor regular de $j$, tendremos que $\mathbf{T}^{*} \mathbf{Q}_{k}=j^{-1}(k) / \mathbb{R}^{3}$ es una variedad simpléctica con 1-forma simpléctica asociada, definida por $\omega_{k}$, que a su vez está definida por medio de la siguiente relación

$$
\pi_{k}^{*} \omega_{k}=i_{k}^{*} \omega
$$

donde $\pi_{k}: j^{-1}(k) \rightarrow \mathbf{T}^{*} \mathbf{Q}_{k}$ es la proyección canónica, $i_{k}: j^{-1}(k) \rightarrow \mathbf{T}^{*} \mathbf{Q}$ es la inclusión canónica, $\pi_{k}^{*} \omega_{k}$ es el pull-back de $\omega_{k}$ por $\pi_{k}$ e $i_{k}^{*} \omega$ es el pull-back de $\omega$ por $i_{k}$. Más adelante. se describirá un modelo para $\mathbf{T}^{*} \mathbf{Q}_{k}$, mediante lo que llamaremos la cadena baricéntrica, que no es si no, una generalización de las coordenadas de Jacobi para $n+1$ sólidos

Definición 33 Sean $\mathbf{R}_{i, j}=\mathbf{R}_{j}-\mathbf{R}_{i}$ los vectores mutuos entre los centros de masas de $S_{i}$ y $S_{j}, \mathbf{G}_{k}$ los centros de masas de los sistemas $\left\{S_{n}, S_{n-1}, \ldots, S_{n-k}\right\}(k=1,2, \ldots, n-1)$. Definimos lo que llamaremos coordenadas baricéntricas mediante

$$
\begin{gathered}
\rho_{1}=\mathbf{R}_{n, n-1}, \quad \rho_{2}=\mathbf{R}_{n-2}-\mathbf{G}_{1}, \quad \rho_{3}=\mathbf{R}_{n-3}-\mathbf{G}_{2} \\
\ldots, \quad \rho_{k}=\mathbf{R}_{n-k}-\mathbf{G}_{k-1}, \quad \rho_{n}=\mathbf{R}_{0}-\mathbf{G}_{n-1}
\end{gathered}
$$

y los $M_{j}=\sum_{k=j-1}^{n} m_{k},(j=1, \ldots, n)$.

Definición 34 Se llaman masas reducidas a las siguientes

$$
g_{1}=\frac{m_{n} m_{n-1}}{M_{n}}, \ldots, \quad g_{i}=\frac{m_{n-i} M_{n-i+2}}{M_{n-i+1}}, \quad \ldots, \quad g_{n}=\frac{m_{0} M_{2}}{M_{1}}
$$


Debido a las definiciones anteriores, los momentos lineales quedan expresados como sigue

$$
\widetilde{\rho_{1}}=g_{1} \dot{\rho_{1}}, \quad \tilde{\rho}_{2}=g_{2} \dot{\rho_{2}}, \quad \widetilde{\rho}_{3}=g_{3} \dot{\rho_{3}}, \quad \ldots, \quad \tilde{\rho}_{i}=g_{i} \dot{\rho_{i}}, \quad \ldots, \quad \tilde{\rho}_{n}=g_{n} \dot{\rho_{n}}
$$

Consideremos la siguiente variedad

$$
\mathbf{M}=\mathbf{S O}(3) \times \mathbb{R}^{3} \times \mathbf{T}^{*} \mathbb{R}^{3} \times \stackrel{n)}{.} \times \mathbf{T}^{*} \mathbb{R}^{3}
$$

y la forma simpléctica $\left.\omega_{\mathbf{M}}=\omega^{\mathbf{S O}(3)}+\omega^{\mathbb{R}^{3}}+. . n\right)+\omega$, la aplicación

$$
\Psi_{1}:\left(\mathbf{T}^{*} \mathbf{Q}_{k}, \omega_{k}\right) \rightarrow\left(\mathbf{M}, \omega_{\mathbf{M}}\right)
$$

definida como

$$
\Psi_{1}(\Xi)=\left(B, B \widehat{\Pi}, \rho_{1}, \widetilde{\rho_{1}}, \rho_{2}, \widetilde{\rho_{2}}, \ldots, \rho_{i}, \widetilde{\rho}_{i}, \ldots, \rho_{n}, \widetilde{\rho_{n}}\right)
$$

es un difeomorfismo simpléctico.

El corchete de Poisson $\{f, g\}_{M}(\mathbf{m})$ asociado a dicha forma simpléctica $\omega_{\mathbf{M}}$ viene dado por

$$
\left(<D_{B} f, \frac{\partial g}{\partial B \widehat{\Pi}}>-<D_{B} g, \frac{\partial f}{\partial B \widehat{\boldsymbol{\Pi}}}>+\sum_{i=1}^{n}\left(\frac{\partial f}{\partial \rho_{i}} \frac{\partial g}{\partial \widetilde{\rho}_{i}}-\frac{\partial g}{\partial \rho_{i}} \frac{\partial f}{\partial \widetilde{\rho}_{i}}\right)\right)(\mathbf{m})
$$

para cualesquiera $f, g \in C^{\infty}(\mathbf{M})$.

El hamiltoniano $\mathcal{H}$ es $\Phi_{1}^{\mathbf{T}^{*} \mathbf{Q}}$-invariante. Por tanto, la proyección $\pi_{k}$ induce un hamiltoniano $\mathcal{H}_{k}$ en $\mathbf{T}^{*} \mathbf{Q}_{k}$ determinado por

$$
\mathcal{H}_{k}\left(\pi_{k}(\mathbf{m})\right)=\mathcal{H}\left(i_{k}(\mathbf{m})\right)
$$

y usando el difeomorfismo $\Psi_{1}$, tendremos definido el siguiente hamiltoniano $\mathcal{H}_{I}$ en $\mathbf{M}$

$$
\mathcal{H}_{I}(\mathbf{m})=\mathcal{H}_{k}\left(\Psi_{1}^{-1}(\mathbf{m})\right)
$$

cuya expresión explícita está dada por la fórmula

$$
\mathcal{H}_{I}(\mathbf{m})=\sum_{i=1}^{n} \frac{\left|\tilde{\rho}_{i}\right|^{2}}{2 g_{i}}+\frac{1}{2} \boldsymbol{\Pi} \cdot \mathbb{I}^{-1} \boldsymbol{\Pi}-\mathbf{l}_{r} \cdot \mathbb{I}^{-1} \boldsymbol{\Pi}+V(\mathbf{m})
$$

donde

$$
V(\mathbf{m})=-\left(\sum_{\substack{i, j=1 \\ i \neq j}}^{n} \frac{G m_{i} m_{j}}{\left|\mathbf{R}_{i, j}\right|}+\sum_{i=1}^{n} \int_{S_{o}} \frac{G m_{i}}{\left|B \mathbf{Q}+\mathbf{R}_{i, 0}\right|} d m(\mathbf{Q})\right)
$$

siendo las $\mathbf{R}_{i, j}$ funciones lineales de $\rho_{i}(i=1, \ldots, n)$.

En particular, para el caso en que $n=2$ tendremos que

$$
\mathbf{R}_{2,1}=\rho_{1}, \quad \mathbf{R}_{1,0}=\rho_{2}-\frac{m_{2}}{M_{2}} \rho_{1}, \quad \mathbf{R}_{2,0}=\rho_{2}+\frac{m_{1}}{M_{2}} \rho_{1}
$$

con $M_{2}=m_{1}+m_{2}$.

Y para $n=3$ obtenemos

$$
\begin{gathered}
\mathbf{R}_{2,1}=\rho_{2}-\frac{m_{3}}{M_{3}} \rho_{1}, \quad \mathbf{R}_{1,0}=\rho_{3}-\frac{M_{3}}{M_{2}} \rho_{2} \\
\mathbf{R}_{3,1}=\rho_{2}+\frac{m_{2}}{M_{3}} \rho_{1}, \quad \mathbf{R}_{2,0}=\rho_{3}+\frac{m_{1}}{M_{2}} \rho_{2}-\frac{m_{3}}{M_{3}} \rho_{1} \\
\mathbf{R}_{3,2}=\rho_{1}, \quad \mathbf{R}_{3,0}=\rho_{3}+\frac{m_{1}}{M_{2}} \rho_{2}+\frac{m_{2}}{M_{3}} \rho_{1}
\end{gathered}
$$

siendo ahora $M_{3}=m_{3}+m_{2}$ y $M_{2}=m_{1}+m_{2}+m_{3}$. 


\subsubsection{Reducción por medio del grupo de rotaciones $\mathrm{SO}(3)$}

Sea la aplicación

$$
\Phi_{2}: \mathbf{S O}(3) \times \mathbf{M} \rightarrow \mathbf{M}
$$

definida para $(A, \mathbf{m}) \in \mathbf{S O}(3) \times \mathbf{M}$ como

$$
\begin{gathered}
\Phi_{2}(A, \mathbf{m})=\left(A B, A B \widehat{\Pi}, A \rho_{1}, A \widetilde{\rho_{1}}, A \rho_{2}, A \widetilde{\rho_{2}}, \ldots\right. \\
\left.\ldots, A \rho_{i}, A \widetilde{\rho_{i}}, \ldots, A \rho_{n}, A \widetilde{\rho_{n}}\right)
\end{gathered}
$$

Esta apliación es una acción libre y propia del grupo $\mathbf{S O}(3)$ en la variedad $\mathbf{M}$. Así pues, $\Phi_{2}$ induce una estructura de Poisson en $\mathbf{M} / \mathbf{S O}(3)$, con corchetes de Poisson dados por

$$
\{f, g\}_{\mathbf{M} / \mathbf{S O}(3)} \circ \pi_{2}=\left\{f \circ \pi_{2}, g \circ \pi_{2}\right\}_{\mathbf{M}}
$$

Por otro lado, el hamiltoniano $\mathcal{H}_{I}$ es $\Phi_{2}^{\mathbf{M} / \mathbf{S O}(3)}$-invariante, la dinámica generalizada generada por $\mathcal{H}_{I}$, induce la dinámica generada por el siguiente hamiltoniano reducido

$$
\mathcal{H}_{I I}\left(\pi_{2}(\mathbf{m})\right)=\mathcal{H}_{I}(\mathbf{m}) \text {. }
$$

Describamos ahora un modelo para la variedad $\mathbf{M} / \mathbf{S O}(3)$. Para ello definimos función

$$
\Psi_{2}:\left(M / \mathbf{S O}(3),\{\cdot, \cdot\}_{M / \mathbf{S O}(3)}\right) \rightarrow\left(\mathbb{R}^{6 n+3},\{\cdot, \cdot\}_{I I}\right)
$$

con

$$
\Psi_{2}(\mathbf{m})=\left(\boldsymbol{\Pi}, \lambda_{1}, \mathbf{p}_{\lambda_{1}}, \lambda_{2}, \mathbf{p}_{\lambda_{2}}, \ldots, \lambda_{n}, \mathbf{p}_{\lambda_{n}}\right)
$$

donde $\lambda_{i}=B^{t} \rho_{i}, \mathbf{p}_{\lambda_{i}}=B^{t} \widetilde{\rho}_{i}(i=1, \ldots, n)$; siendo $\Psi_{2}$ un difeomorfismo de Poisson con corchete de Poisson $\{\cdot, \cdot\}_{I I}$ dado por

$$
\{f, g\}_{I I}(\mathbf{z})=\left(\nabla_{z} f\right)^{t} \mathbf{B}(\mathbf{z}) \nabla_{z} g
$$

y con el tensor de Poisson $\mathbf{B}(\mathbf{z})$ dado por la siguiente expresión

$$
\mathbf{B}(\mathbf{z})=\left(\begin{array}{ccccccc}
\widehat{\boldsymbol{\Pi}} & \widehat{\lambda_{1}} & \widehat{\mathbf{p}_{\lambda_{1}}} & \cdots & \ldots & \widehat{\lambda_{n}} & \widehat{\mathbf{p}_{\lambda_{n}}} \\
\widehat{\lambda_{1}} & \mathbf{0} & I_{\mathbb{R}^{3}} & \mathbf{0} & \ldots & \ldots & \mathbf{0} \\
\widehat{\mathbf{p}_{\lambda_{1}}} & -I_{\mathbb{R}^{3}} & \mathbf{0} & \ddots & \ddots & \ldots & \cdots \\
\vdots & \vdots & \ddots & \ddots & \ddots & \ddots & \cdots \\
\vdots & \vdots & \vdots & \ddots & \ddots & \ddots & \mathbf{0} \\
\widehat{\lambda_{n}} & \mathbf{0} & \vdots & \vdots & \ddots & \mathbf{0} & I_{\mathbb{R}^{3}} \\
\widehat{\mathbf{p}_{\lambda_{n}}} & \mathbf{0} & \vdots & \vdots & \mathbf{0} & -I_{\mathbb{R}^{3}} & \mathbf{0}
\end{array}\right)
$$

El hamiltoniano doblemente reducido es

$$
\mathcal{H}_{I I}(\mathbf{z})=\mathcal{H}_{\mathbf{M} / \mathbf{S O}(3)}\left(\Psi_{2}^{-1}(\mathbf{z})\right)=\sum_{i=1}^{n} \frac{\left|\mathbf{p}_{\lambda_{i}}\right|^{2}}{2 g_{i}}+\frac{1}{2} \Pi \Pi^{-1} \Pi-\mathbf{l}_{r} \cdot \mathbb{I}^{-1} \Pi+V(\mathbf{z})
$$

cuyo potencial viene dado por

$$
V(\mathbf{z})=-\left(\sum_{\substack{i, j=1 \\ i \neq j}}^{n} \frac{G m_{i} m_{j}}{\left|\mathbf{R}_{i, j}\right|}+\sum_{i=1}^{n} \int_{S_{o}} \frac{G m_{i}}{\left|\mathbf{Q}+B^{t} \mathbf{R}_{i, 0}\right|} d m(\mathbf{Q})\right)
$$

con $B^{t} \mathbf{R}_{i, j}$ expresado como funciones de $\lambda_{i}(i, j=1, \ldots, n)$. 


\subsection{Expresión de la energía potencial}

La energía potencial en el caso del problema de dos cuerpos adopta la forma

$$
\begin{gathered}
V\left(\lambda_{1}, \lambda_{2}\right)=-\left(\frac{G m_{1} m_{2}}{\left|\lambda_{1}\right|}+G m_{1} \int_{S_{0}} \frac{d m(\mathbf{Q})}{\left|\mathbf{Q}+\lambda_{2}-\frac{m_{2}}{M_{2}} \lambda_{1}\right|}+\right. \\
\left.G m_{2} \int_{S_{0}} \frac{d m(\mathbf{Q})}{\left|\mathbf{Q}+\lambda_{2}+\frac{m_{1}}{M_{2}} \lambda_{2}\right|}\right)
\end{gathered}
$$

En tanto que para el problema de tres cuerpos adopta ahora la forma

$$
\begin{gathered}
V\left(\lambda_{1}, \lambda_{2}, \lambda_{3}\right)=-\left(\frac{G m_{2} m_{3}}{\left|\lambda_{1}\right|}+\frac{G m_{1} m_{2}}{\left|\lambda_{2}-\frac{m_{3}}{M_{3}} \lambda_{1}\right|}+\frac{G m_{1} m_{3}}{\left|\lambda_{2}+\frac{m_{2}}{M_{3}} \lambda_{1}\right|}+\right. \\
G m_{1} \int_{S_{0}} \frac{d m(\mathbf{Q})}{\left|\mathbf{Q}+\lambda_{\mathbf{3}}-\frac{M_{3}}{M_{2}} \lambda_{2}\right|}+G m_{2} \int_{S_{0}} \frac{d m(\mathbf{Q})}{\left|\mathbf{Q}+\lambda_{\mathbf{3}}+\frac{m_{1}}{M_{2}} \lambda_{2}-\frac{m_{3}}{M_{3}} \lambda_{1}\right|} \\
\left.+G m_{3} \int_{S_{0}} \frac{d m(\mathbf{Q})}{\left|\mathbf{Q}+\lambda_{\mathbf{3}}+\frac{m_{1}}{M_{2}} \lambda_{2}+\frac{m_{2}}{M_{3}} \lambda_{1}\right|}\right)
\end{gathered}
$$

Ambas expresiones pueden generalizarse para cualquier número de cuerpos rígidos, pero resolver las anteriores integrales, exceptuando el caso en el que encontremos ciertas simetrías del giróstato $S_{0}$, son muy complicadas de resolver. Es por ello, que resulta mejor, considerar el desarrollo multipolar de dichos potenciales, suponiendo que los cuerpos involucrados están a distancias mutuas mucho mayores que sus propias dimensiones. Bajo ciertas hipótesis adicionales, se podrá desarrollar el potencial en series rápidamente convergentes Leimanis (1965) [34]. Por último, considerando los potenciales truncados hasta cierto orden arbitraro, estaremos en condiciones de estudiar la dinámica aproximada del hamiltoniano.

Para un giróstato triaxial a gran distancia la siguiente fórmula se cumple con una aproximación bastante buena, en el caso en que $n=2$,

$$
V=V_{1}+V_{2}
$$

donde

$$
\begin{gathered}
V_{1}=-\left(\frac{G m_{1} m_{2}}{\left|\lambda_{1}\right|}+\frac{G m_{1} m_{0}}{\left|\lambda_{2}-\frac{m_{2}}{M_{2}} \lambda_{1}\right|}+\frac{G m_{2} m_{0}}{\left|\lambda_{2}+\frac{m_{1}}{M_{2}} \lambda_{1}\right|}\right) \\
V_{2}=-\frac{1}{2}\left(\frac{G m_{1} \alpha}{\left|\lambda_{2}-\frac{m_{2}}{M_{2}} \lambda_{1}\right|^{3}}+\frac{G m_{2} \alpha}{\left|\lambda_{2}+\frac{m_{1}}{M_{2}} \lambda_{1}\right|^{3}}-\frac{3 G m_{1} \beta_{1}}{\left|\lambda_{2}-\frac{m_{2}}{M_{2}} \lambda_{1}\right|^{5}}-\frac{3 G m_{2} \beta_{2}}{\left|\lambda_{2}+\frac{m_{1}}{M_{2}} \lambda_{1}\right|^{5}}\right)
\end{gathered}
$$


y

$$
\begin{gathered}
\alpha=A+B+C \\
\beta_{1}\left(\lambda_{1}, \lambda_{2}\right)=\lambda_{2} \cdot \mathbb{I} \lambda_{2}-\frac{2 m_{2}}{M_{2}} \lambda_{1} \cdot \mathbb{I} \lambda_{2}+\left(\frac{m_{2}}{M_{2}}\right)^{2} \lambda_{1} \cdot \mathbb{I} \lambda_{1} \\
\beta_{2}\left(\lambda_{1}, \lambda_{2}\right)=\lambda_{2} \cdot \mathbb{I} \lambda_{2}+\frac{2 m_{1}}{M_{2}} \lambda_{1} \cdot \mathbb{I} \lambda_{2}+\left(\frac{m_{1}}{M_{2}}\right)^{2} \lambda_{1} \cdot \mathbb{I} \lambda_{1}
\end{gathered}
$$

El siguiente resultado, explica el potential creado por un sólido o giróstato en un punto $P$ a gran distancia, bajo ciertas condiciones de simetría Leimanis (1965) [34].

Proposición 35 Consideremos un giróstato $S_{0}$ de masa $m_{0}$; entonces, el potencial creado por $S_{0}$ en un punto material externo $P$, de masa $\widetilde{m}$, viene dado por la siguiente expresión

$$
V=-\frac{G \widetilde{m}}{r} \sum_{n=0}^{\infty} \frac{1}{r^{n}} A_{n}
$$

donde $r=|\mathbf{r}|$ es la distancia desde el centro de masas del giróstato a $P$ y los coeficientes $A_{n}$ vienen dados por

$$
A_{n}=\int_{S} r^{\prime n} P_{n}\left(\frac{\mathbf{r} \cdot \mathbf{r}^{\prime}}{r r^{\prime}}\right) d m
$$

siendo $\mathbf{r}^{\prime}$ el vector posición relativo al centro de masas de $S_{0}$ para cualquier partícula $P^{\prime} \in S_{0}, r^{\prime}=\left|\mathbf{r}^{\prime}\right| y$

$$
P_{n}(x)=\frac{1}{n ! 2^{n}} \frac{d^{n}\left[\left(x^{2}-1\right)^{n}\right]}{d x^{n}}
$$

los polinomios de Legendre de grado n. En particular, cuando $S_{0}$ es simétrico alrededor de eje $O Z$ y respecto al plano $O X Y$ su tensor de inercia viene dado por la matriz

$$
\mathbb{I}=\left(\begin{array}{ccc}
A & 0 & 0 \\
0 & A & 0 \\
0 & 0 & C
\end{array}\right)
$$

y las siguientes relaciones se verifican

$$
A_{1}=A_{3}=A_{5}=A_{7}=\ldots=0
$$

En particular, $A_{0}=m_{0}, A_{2}=(C-A) / 2$ y el potencial puede aproximarse con gran precisión por medio de la fórmula de MacCullagh

$$
V \simeq-\frac{G m_{0} \tilde{m}}{r}-\frac{G \widetilde{m}(C-A)}{2 r^{3}}
$$

Corolario 36 Aplicando los resultados previos al caso $n=2$, obtenemos la siguiente expresión para el potencial

$$
\begin{aligned}
V\left(\lambda_{1}, \lambda_{2}\right)= & -\left(\frac{G m_{1} m_{2}}{\left|\lambda_{1}\right|}+G m_{1} \sum_{i=0}^{\infty} \frac{A_{2 i}}{\left|\lambda_{\mathbf{2}}-\frac{m_{2}}{M_{2}} \lambda_{1}\right|^{2 i+1}}\right. \\
& \left.+G m_{2} \sum_{i=0}^{\infty} \frac{A_{2 i}}{\left|\lambda_{\mathbf{2}}+\frac{m_{1}}{M_{2}} \lambda_{1}\right|^{2 i+1}}\right)
\end{aligned}
$$

siendo $A_{0}=m_{0}, A_{2}=(C-A) / 2$ y $A_{2 i}$ dados por (3.37).

Observación 37 Resultados similares pueden ser obtenidos para el caso en que $n=3$ o más cuerpos. 


\subsection{Ecuaciones del movimiento}

Las ecuaciones del movimiento pueden escribirse como

$$
\dot{\mathbf{z}}=\left\{\mathbf{z}, \mathcal{H}_{I I}(\mathbf{z})\right\}_{I I}(\mathbf{z})=\mathbf{B}(\mathbf{z}) \nabla_{\mathbf{z}} \mathcal{H}_{I I}(\mathbf{z})
$$

Desarrollando la fórmula anterior obtenemos las ecuaciones diferenciales del movimiento

$$
\begin{gathered}
\dot{\Pi}=\boldsymbol{\Pi} \times \Omega+\sum_{i=1}^{n} \lambda_{i} \times \nabla_{\lambda_{i}} V \\
\dot{\lambda_{i}}=\lambda_{i} \times \Omega+\frac{1}{g_{i}} \mathbf{p}_{\lambda_{i}}, \quad \dot{\mathbf{p}_{\lambda_{i}}}=\mathbf{p}_{\lambda_{i}} \times \Omega-\nabla_{\lambda_{i}} V
\end{gathered}
$$

donde $\boldsymbol{\Omega}=\mathbb{I}^{-1}\left(\boldsymbol{\Pi}-\mathbf{l}_{r}\right)$ es la velocidad angular del giróstato $S_{0}$ y $\nabla_{\lambda_{i}} V$ es la derivada parcial de $V$ con respecto a la variable $\lambda_{i}$.

\subsubsection{Funciones de Casimir. Integrales del momento angular}

Elementos importantes de $\mathbf{B}(\mathbf{z})$ son los asociados a las funciones de Casimir. Al igual que en Maciejewski (1995) [37], Mondéjar el al (2001) [48] y Vera y Vigueras (2004) [60]. Consideremos el momento angular total $\mathbf{L}$ dado por:

$$
\mathbf{L}=\mathbf{\Pi}+\sum_{i=1}^{n} \lambda_{i} \times \mathbf{p}_{\lambda_{i}}
$$

Por tanto, podemos probar el siguiente resultado:

Proposición 38 Si $\varphi$ es una función real suficientemente diferenciable no constante, entonces $\varphi\left(\frac{|\mathbf{L}|^{2}}{2}\right)$ es una función Casimir del tensor de Poisson $\mathbf{B}(\mathbf{z})$. Es más, tenemos que $\operatorname{Ker} \mathbf{B}(\mathbf{z})=\left\langle\nabla_{\mathbf{z}} \varphi>\right.$. También se tiene $\frac{d \mathbf{L}}{d t}=\mathbf{0}$, esto nos dice que el vector momento angular total se mantiene constante.

\subsubsection{Equilibrios relativos}

Las ecuaciones que definen los equilibrios relativos del sistema, vienen dadas por el siguiente sistema homogéneo

$$
\begin{gathered}
\boldsymbol{\Pi} \times \boldsymbol{\Omega}+\sum_{i=1}^{n} \lambda_{i} \times \nabla_{\lambda_{i}} V=\mathbf{0} \\
\lambda_{i} \times \mathbf{\Omega}+\frac{1}{g_{i}} \mathbf{p}_{\lambda_{i}}=\mathbf{0}, \quad \mathbf{p}_{\lambda_{i}} \times \mathbf{\Omega}-\nabla_{\lambda_{i}} V=\mathbf{0}
\end{gathered}
$$

Denotamos por $\mathbf{z}_{e}=\left(\boldsymbol{\Pi}_{e}, \lambda_{1}^{e}, \mathbf{p}_{\lambda_{1}}^{e}, \ldots, \lambda_{i}^{e}, \mathbf{p}_{\lambda i}^{e}, \ldots, \lambda_{n}^{e}, \mathbf{p}_{\lambda_{n}}^{e}\right)$ al equilibrio relativo de las ecuaciones de movimiento (3.44), y aplicando ls propiedades del producto vectorial obtenemos el siguiente resultado: 
Proposición 39 Si $\mathbf{z}_{e}$ es un equilibrio relativo de las ecuaciones (3.44), entonces

$$
\begin{gathered}
\boldsymbol{\Pi}_{e} \times \boldsymbol{\Omega}_{e}+\sum_{i=1}^{n} \lambda_{i}^{e} \times \nabla_{\lambda_{i}} V_{e}=0 \\
\lambda_{i}^{e}\left|\boldsymbol{\Omega}_{e}\right|^{2}-\left(\lambda_{i}^{e} \cdot \boldsymbol{\Omega}_{e}\right) \boldsymbol{\Omega}_{e}=\frac{1}{g_{i}} \nabla_{\lambda_{i}} V_{e}
\end{gathered}
$$

donde el subíndice e se usa para indicar que la correspondiente variable o función es evaluada en el punto $\mathbf{z}_{e} y \boldsymbol{\Omega}_{e}$ es la velocidad angular de $S_{0}$ en el equilibrio relativo $\mathbf{z}_{e}$.

Realizando el producto escalar en las expresiones anteriores con el correspondiente $\lambda_{i}^{e}$, obtenemos el siguiente corolario.

Corolario 40 Si $\mathbf{z}_{e}$ es un equilibrio relativo de las ecuaciones (3.44), entonces

$$
\left|\Omega_{e}\right|^{2}\left|\lambda_{i}^{e}\right|^{2}-\left(\lambda_{i}^{e} \cdot \Omega_{e}\right)^{2}=\frac{1}{g_{i}}\left(\lambda_{i}^{e} \cdot \nabla_{\lambda_{i}} V_{e}\right)(i=1, \ldots, n)
$$

Observación 41 De la última ecuación, el valor de $\left|\Omega_{e}\right|$ puede ser deducido en un equilibrio relativo.

\subsubsection{Carácter variacional del los equilibrios relativos}

Una condición necesaria y suficiente para que $\mathbf{z}_{e}$ sea un equilibrio relativos de las ecuaciones de moviiento (3.44), es que existe $\lambda \in \mathbb{R}$ tal que

$$
\mathbf{d}\left(\mathcal{H}_{I I}+\lambda\left(\frac{1}{2}|\mathbf{L}|^{2}\right)\right)\left(\mathbf{z}_{e}\right)=\mathbf{0}
$$

de eso deducimos las ecuaciones

$$
\begin{gathered}
\boldsymbol{\Omega}_{e}+\lambda \mathbf{L}_{e}=\mathbf{0} \\
\nabla_{\lambda_{i}} V_{e}+\lambda\left(\mathbf{p}_{\lambda_{i}}^{e} \times \mathbf{L}_{e}\right)=\mathbf{0}, \quad \frac{\mathbf{p}_{\lambda_{i}}^{e}}{g_{i}}-\lambda\left(\lambda_{i}^{e} \times \mathbf{L}_{e}\right)=\mathbf{0}
\end{gathered}
$$

Entonces la siguiente igualdad se verifica

$$
\left|\Omega_{e}\right|^{2}=\lambda^{2}\left|\mathbf{L}_{e}\right|^{2}
$$

Observación 42 Obsérvese que las ecuaciones mencionadas arriba son equivalentes a las ecuaciones que determinan los equilibrios relativos del sistema (3.46).

Así pues, tenemos la siguiente propiedad.

Proposición 43 Si $\mathbf{z}_{e}$ es un equilibrio relativo de las eccuaciones (3.44), la velocidad angular $\boldsymbol{\Omega}_{e}$ de $S_{0}$, es paralela al momento angular total del sistema $\mathbf{L}$. 


\subsection{Variedades invariantes}

Es fácil verificar, denotando por $\lambda_{i}=\left(\lambda_{i}^{1}, \lambda_{i}^{2}, \lambda_{i}^{3}\right)$, que

$$
\mathbf{M}_{C}=\left\{\mathbf{z} \in \mathbb{R}^{6 n+3} / \pi_{1}=\pi_{2}=\lambda_{i}^{3}=p_{\lambda_{i}^{3}}=0(i=1, \ldots, n)\right\}
$$

es una variedad invariante para el flujo (3.44). De manera similar se puede obtener $\mathbf{M}_{B}$, $\mathbf{M}_{A}$.

A través de un cálculo directo se puede verificar el siguiente resultado

Proposición $44 \mathbf{M}_{C}, \mathbf{M}_{B}$ y $\mathbf{M}_{A}$ son variedades invariantes del flujo definido por las ecuaciones del movimiento (3.43). Considerando $\mathbf{B}(\mathbf{z})$ restringido a $\mathbf{M}_{C}$, y denotando su restricción como $\mathbf{B}_{\mathbf{M}_{C}}(\mathbf{z})$, tenemos que $L_{\mathbf{M}_{C}}=\pi_{3}+\sum_{i=1}^{n}\left(\lambda_{i}^{1} p_{\lambda_{i}^{2}}-\lambda_{i}^{2} p_{\lambda_{i}^{1}}\right)$ es una función Casimir del tensor de Poisson $\mathbf{B}_{\mathbf{M}_{C}}(\mathbf{z})$ y $\operatorname{Ker} \mathbf{B}_{\mathbf{M}_{C}}(\mathbf{z})=<\nabla_{\mathbf{z}} L_{\mathbf{M}_{C}}>$.

Observación 45 Resultados similares se pueden obtener para $\mathbf{B}_{\mathbf{M}_{A}}(\mathbf{z})$ y $\mathbf{B}_{\mathbf{M}_{B}}(\mathbf{z})$.

Las ecuaciones (3.44) restringidas a $\mathbf{M}_{C}$ son equivalentes a aquellas obtenidas por medio del hamiltoniano canónico del sistema $\left(\mathbb{R}^{4 n}, \mathcal{H}, \omega^{\mathbb{R}^{4 n}}\right)$ donde el hamiltoniano $\mathcal{H}$ viene dado por la siguiente expresión

$$
\mathcal{H}=\sum_{i=1}^{n} \frac{\left|\mathbf{p}_{\lambda_{i}}\right|^{2}}{2 g_{i}}+\frac{1}{2 C} K\left(\lambda_{i}, \mathbf{p}_{\lambda_{i}}\right)^{2}-\frac{l}{C} K\left(\lambda_{i}, \mathbf{p}_{\lambda_{i}}\right)+V(\mathbf{z})
$$

con $K\left(\lambda_{i}, \mathbf{p}_{\lambda_{i}}\right)=L_{\mathbf{M}_{C}}-\sum_{i=1}^{n}\left(\lambda_{i}^{1} p_{\lambda_{i}^{2}}-\lambda_{i}^{2} p_{\lambda_{i}^{1}}\right)$ y $\omega^{\mathbb{R}^{4 n}}$ la forma simpléctica estándard en $\mathbb{R}^{4 n}$.

Vemamos ahora el siguiente resultado

Proposición 46 Si $\mathbf{z}_{e}$ es un equilibrio relativo del sistema de Lie-Poisson $\left(\mathbb{R}^{4 n}, \mathcal{H}, \omega^{\mathbb{R}^{4 n}}\right)$, entonces $\mathbf{z}_{e}$ es un equilibrio relativo de $\left(\mathbb{R}^{6 n+3}, \mathcal{H}_{I I}, \mathbf{B}(\mathbf{z})\right)$. Si $\mathbf{z}_{e}$ es un equilibrio relativo de $\left(\mathbb{R}^{6 n+3}, \mathcal{H}_{I I}, \mathbf{B}(\mathbf{z})\right)$, siendo $\mathbf{z}_{e} \in \mathbf{M}_{C}$, entonces $\mathbf{z}_{e}$ es un equilibrio relativo de $\left(\mathbb{R}^{4 n}, \mathcal{H}, \omega^{\mathbb{R}^{4 n}}\right)$.

Observación 47 Resultados similares se pueden obtener para las variedades invariantes $\mathbf{M}_{A}$ y $\mathbf{M}_{B}$.

\subsubsection{Movimiento plano}

Ahora las ecuaciones del movimiento vienen dadas por

$$
\dot{\lambda_{i}}=\nabla_{\mathbf{p}_{\lambda_{i}}} \mathcal{H}, \quad \dot{\mathbf{p}_{\lambda_{i}}}=-\nabla_{\lambda_{i}} \mathcal{H}(i=1, \ldots, n)
$$

y describen el movimiento plano de un giróstato en la variedad invariante $\mathbf{M}_{C}$. Como ya hemos comentado antes, resultados similares se obtienen si en vez de restringirnos a la variedad $\mathbf{M}_{C}$ usamos las variedades $\mathbf{M}_{A}$ y $\mathbf{M}_{B}$. 



\section{4 \\ Dinámica hamiltoniana de un giróstato en interacción newtoniana con un sólido rígido esférico}

\subsection{Introducción}

En este capítulo, partiendo del sistema hamiltoniano no canónico que describe la dinámica de un giróstato en interacción newtoniana con un sólido rígido esférico o partícula material, deduciremos la dinámica hamiltoniana canónica que caracteriza a dicho sistema en las tres variedades invariantes del movimiento que existen. Obtendremos asimismo el potencial ampliado y las regiones de Hill, muy útiles para posteriores estudios cualitativos sobre la dinámica de un sistema giróstato-esfera.

\subsection{Ecuaciones del movimiento}

Siguiendo con la notación introducida en los capítulos anteriores, llamaremos:

- $S_{0}$ es un giróstato de masa $m_{0}$ (o masa puntual).

- $S_{1}$ un cuerpo rígido esférico de masa $m_{1}$.

- $M_{1}=m_{0}+m_{1}$.

- $g_{1}=m_{0} m_{1} / M_{1}$.

- $\mathbf{I}_{\mathbb{R}^{3}}$ es la matriz identidad y $\mathbf{0}$ es la matriz nula de orden tres.

- La matriz diagonal de orden tres $\mathbb{I}=\operatorname{diag}(A, B, C)$ es el tensor diagonal de inercia asociado al giróstato $S_{0}$.

- El vector $\mathbf{z}=(\boldsymbol{\Pi}, \mathbf{u}, \mathbf{p}) \in \mathbb{R}^{9}$ es un elemento del espacio de fase doblemente reducido obtenido al usar las simetrías del sistema, siendo mathbfu el vector que va del centro de masas de $S_{1}$ al centro de masas de $S_{0}$ y $\mathbf{P}$ el momento lineal.

- $\Omega$ el vector velocidad angular de $S_{0}$. 
- $\boldsymbol{\Pi}=\mathbb{I} \boldsymbol{\Omega}+\mathbf{l}_{r}$ el vector momento angular total del giróstato en el sistema de referencia ligado al cuerpo, cuyos ejes tienen la dirección de los ejes principales de inercia de $S_{0}$.

- Suponemos, para simplificar, que el momento girostático $\mathbf{l}_{r}=(0,0, l)$ debido al movimiento relativo de las partes móviles del giróstato respecto a las rígidas es constante y paralelo al tercer eje de inercia .

- Los elementos $\mathbf{u}, \mathbf{p}$ son las coordenadas y momentos lineales asociados a la reducción al centro de masas del sistema, expresados en el sistema de referencia ligado al cuerpo $\mathfrak{J}$.

- Por último $\mathbf{L}=\boldsymbol{\Pi}+\mathbf{u} \times \mathbf{p}$ es el momento angular total del sistema.

Sea $\mathbf{M}=\mathbb{R}^{9}$, y consideramos el siguiente sistema de Lie-Poisson $(\mathbf{M},\{\},, \mathcal{H})$, con los corchetes de Poisson $\{$,$\} definidos por medio del tensor de Poisson$

$$
\mathbf{B}(\mathbf{z})=\left(\begin{array}{ccc}
\widehat{\Pi} & \widehat{\mathbf{u}} & \widehat{\mathbf{p}} \\
\widehat{\mathbf{u}} & \mathbf{0} & I_{\mathbb{R}^{3}} \\
\widehat{\mathbf{p}} & -I_{\mathbb{R}^{3}} & \mathbf{0}
\end{array}\right)
$$

En cada $\mathbf{B}(\mathbf{z})$, la matriz $\widehat{v}$ es la imagen del vector $\mathbf{v} \in \mathbb{R}^{3}$ por el isomorfismo estándar entre las álgebras de Lie $\mathbb{R}^{3}$ y $\mathfrak{s o}(3)$, es decir

$$
\widehat{\mathbf{v}}=\left(\begin{array}{ccc}
0 & -v_{3} & v_{2} \\
v_{3} & 0 & -v_{1} \\
-v_{2} & v_{1} & 0
\end{array}\right)
$$

El hamiltoniano doblemente reducido del sistema (véase el capítulo del Problema de $n$ cuerpos), tiene la siguiente expresión

$$
\mathcal{H}(\mathbf{z})=\frac{|\mathbf{p}|^{2}}{2 g_{1}}+\frac{1}{2} \Pi \mathbb{I}^{-1} \Pi-\mathbf{l}_{r} \cdot \mathbb{I}^{-1} \Pi+\mathcal{V}(\mathbf{u}),
$$

donde $\mathcal{V}\left(\mathbf{u}_{1}\right)$ es el potencial de la interacción gravitatoria entre el giróstato $S_{0}$ y el cuerpo esférico $S_{1}$, dado por la fórmula

$$
\mathcal{V}\left(\mathbf{u}_{1}\right)=-\int_{S_{0}} \frac{G m_{1} d m(\mathbf{Q})}{\left|\mathbf{Q}+\frac{m_{1}}{M_{1}} \mathbf{u}\right|} .
$$

Las ecuaciones del movimiento vienen dadas por

$$
\frac{d \mathbf{z}}{d t}=\{\mathbf{z}, \mathcal{H}(\mathbf{z})\}=\mathbf{B}(\mathbf{z}) \nabla_{\mathbf{z}} \mathcal{H}(\mathbf{z})
$$

por tanto, si desarrollamos $\{\mathbf{z}, \mathcal{H}(\mathbf{z})\}$, obtendremos las siguientes ecuaciones vectoriales del movimiento

$$
\begin{gathered}
\frac{d \boldsymbol{\Pi}}{d t}=\boldsymbol{\Pi} \times \boldsymbol{\Omega}+\mathbf{u} \times \nabla_{\mathbf{u}} \mathcal{V} \\
\frac{d \mathbf{u}}{d t}=\frac{\mathbf{p}}{g_{1}}+\mathbf{u} \times \Omega, \quad \frac{d \mathbf{p}}{d t}=\mathbf{p} \times \Omega-\nabla_{\mathbf{u}} \mathcal{V} .
\end{gathered}
$$




\subsection{Funciones de Casimir. Integrales del momento angular}

Elementos importantes de $\mathbf{B}(\mathbf{z})$ son los asociados a las funciones de Casimir. Consideremos el momento angular total $\mathbf{L}$ dado por

$$
\mathbf{L}=\Pi+\mathbf{u} \times \mathbf{p} .
$$

Por tanto, podemos probar el siguiente resultado:

Proposición 48 Si $\varphi$ es una función real suficientemente diferenciable no constante, entonces $\varphi\left(\frac{|\mathbf{L}|^{2}}{2}\right)$ es una función Casimir del tensor de Poisson $\mathbf{B}(\mathbf{z})$. Es más, tenemos $q u e \operatorname{Ker} \mathbf{B}(\mathbf{z})=\left\langle\nabla_{\mathbf{z}} \varphi>\right.$. También se tiene $\frac{d \mathbf{L}}{d t}=\mathbf{0}$, esto nos dice que el vector momento angular total se mantiene constante.

\subsection{Dinámica hamiltoniana aproximada}

Consideramos el desarrollo multipolar del potencial $\mathcal{V}(\mathbf{u})$, suponiendo que los cuerpos involucrados están en distancias mucho más grandes que las dimensiones individuales de los mismos. Bajo esta hipótesis adicional podremos desarrollar el potencial en una serie rápidamente convergente. Considerando que los potenciales están truncados hasta un orden arbitrario, podremos estudiar la dinámica hamiltoniana aproximada.

Para un giróstato triaxial la fórmula siguiente (véase Vera y Vigueras (2006) [62]), se verifica con gran exactitud

$$
\mathcal{V}_{1}=-\frac{G m_{1}}{|\mathbf{u}|}\left(m_{0}+\frac{\operatorname{trace}(\mathbb{I})}{2|\mathbf{u}|^{2}}-\frac{3(\mathbf{u} \cdot \mathbb{I} \mathbf{u})}{2|\mathbf{u}|^{4}}\right)
$$

Por tanto,

$$
\mathcal{H}_{1}(\mathbf{z})=\frac{|\mathbf{p}|^{2}}{2 g_{1}}+\frac{1}{2} \Pi \mathbb{I}^{-1} \Pi-\mathbf{l}_{r} \cdot \mathbb{I}^{-1} \Pi+\mathcal{V}_{1}(\mathbf{u}) .
$$

Y las ecuaciones del movimiento para esta dinámica aproximada, vienen dadas por la siguiente expresión

$$
\frac{d \mathbf{z}}{d t}=\left\{\mathbf{z}, \mathcal{H}_{1}(\mathbf{z})\right\}=\mathbf{B}(\mathbf{z}) \nabla_{\mathbf{z}} \mathcal{H}_{1}(\mathbf{z}) .
$$

De la ecuación anterior, se deduce que

$$
\nabla_{\mathbf{z}}\left(|\mathbf{L}|^{2}\right) \mathbf{B}(\mathbf{z}) \nabla_{\mathbf{z}} \mathcal{H}_{1}(\mathbf{z})=0 .
$$

Del mismo modo, cuando el giróstato es de revolución, tenemos que

$$
\nabla_{\mathbf{z}}\left(\pi_{3}\right) \mathbf{B}(\mathbf{z}) \nabla_{\mathbf{z}} \mathcal{H}_{1}(\mathbf{z})=0
$$

dónde $\pi_{3}$ es la tercera componente del momento angular del giróstato. Se verifica el siguiente teorema.

Teorema 49 Para las ecuaciones (4.8)| $\left.\mathbf{L}\right|^{2}$ es una integral del movimiento. Y cuando el giróstato es de revolución $\pi_{3}$ será otra integral del movimiento. 


\subsubsection{Variedades invariantes del movimiento}

Denotando por $\mathbf{u}=\left(u_{1}, u_{2}, u_{3}\right), \mathbf{p}=\left(p_{1}, p_{2}, p_{3}\right)$ y $\boldsymbol{\Pi}=\left(\pi_{1}, \pi_{2}, \pi_{3}\right)$, es fácil verificar que

$$
\mathbf{M}_{C}=\left\{\mathbf{z} \in \mathbb{R}^{9} / \pi_{1}=\pi_{2}=u_{3}=p_{3}=0\right\},
$$

es una variedad invariante para el flujo de (4.8). Similares resultados pueden ser obtenidos para las variedades invariantes $\mathbf{M}_{A}, \mathbf{M}_{B}$ en los casos en los que el momento girostático está en el primer o segundo eje de inercia, respectivamente.

Como en Vera y Vigueras (2006) [62], consideremos $\mathbf{B}(\mathbf{z})$ restringido a $\mathbf{M}_{C}$, y denotémosle por $\mathbf{B}_{\mathbf{M}_{C}}(\mathbf{z})$, entonces

$$
L_{\mathbf{M}_{C}}=\pi_{3}+\left(u_{1} p_{2}-u_{2} p_{1}\right)
$$

es el único Casimir del tensor de Poisson $\mathbf{B}_{\mathbf{M}_{C}}(\mathbf{z})$. Y para cada valor del momento angular total del sistema $L_{\mathbf{M}_{C}}=L$ constante, la dinámica en cada una de las fibras $\mathbf{M}_{C} \bigcap$ $\left(L_{M_{C}}=L\right)$ adopta forma canónica, siendo la expresión del hamiltoniano $\mathcal{H}_{1}$

$$
\mathcal{H}_{1}(\mathbf{z})=\frac{|\mathbf{p}|^{2}}{2 g_{1}}+\frac{1}{2 C}\left(L-\left(u_{1} p_{2}-u_{2} p_{1}\right)\right)^{2}-\frac{l}{C}\left(L-\left(u_{1} p_{2}-u_{2} p_{1}\right)\right)+\mathcal{V}_{1}(\mathbf{u})
$$

con $\mathbf{z}=(\mathbf{u}, \mathbf{P})$ siendo ahora $\mathbf{u}=\left(u_{1}, u_{2}\right)$ y $\mathbf{P}=\left(P_{1}, P_{2}\right)$. Donde $l$ es la tercera componente del momento girostático, que suponemos es constante. Y el potencial (4.6), bajo estas hipótesis, adopta la forma

$$
\mathcal{V}_{1}(\mathbf{u})=-\frac{G m_{1}}{\sqrt{u_{1}^{2}+u_{2}^{2}}}\left(m_{0}+\frac{A+B+C}{2\left(u_{1}^{2}+u_{2}^{2}\right)}-\frac{3\left(A u_{1}^{2}+B u_{2}^{2}\right)}{2\left(u_{1}^{2}+u_{2}^{2}\right)^{2}}\right) .
$$

Así pues,

$$
\mathcal{H}_{1}=\frac{p_{1}^{2}+p_{2}^{2}}{2 g_{1}}+\frac{1}{2 C}\left(\gamma-\left(u_{1} p_{2}-u_{2} p_{1}\right)\right)^{2}+\mathcal{V}_{1}(\mathbf{u})
$$

siendo $\gamma=L-l$.

Para simplificar la notación, escalaremos el problema de la siguiente manera: $G=m_{0}+m_{1}=1 \mathrm{y}$ definimos las constantes $\alpha=\sigma / 2 C, \beta=\gamma / \sigma^{2}, \sigma=\mu(1-\mu)$ y $\mu=m_{0}$. También hacemos

$$
\mathbf{u}=\mathbf{U}, \quad \mathbf{p}=\sigma \mathbf{P} .
$$

Esta transformación es simpléctica con multiplicador $\sigma^{-1}$ y el hamiltoniano $\mathcal{H}_{1}$ se reduce a

$$
\mathcal{H}_{1}=\frac{P_{1}^{2}+P_{2}^{2}}{2}+\alpha\left(\beta-\left(U_{1} P_{2}-U_{2} P_{1}\right)\right)^{2}+\frac{1}{\sigma} \mathcal{V}_{1}(\mathbf{U})
$$

Haciendo una transformación canónica de coordenadas cartesianas a polares-simplécticas, el hamiltoniano toma la forma

$$
\mathcal{H}_{1}(\mathbf{z})=\frac{1}{2}\left(p_{r}^{2}+\frac{p_{\theta}^{2}}{r^{2}}\right)+\alpha\left(\beta-p_{\theta}\right)^{2}-\frac{1}{r}\left(1+\frac{A+B+C}{2 r^{2} \mu}-\frac{3\left(A \cos ^{2} \theta+B \sin ^{2} \theta\right)}{2 r^{2} \mu}\right),
$$

siendo ahora $\mathbf{z}=\left(r, \theta, p_{r}, p_{\theta}\right) \in \mathbb{R}^{+} \times S^{1} \times \mathbb{R}^{2}$.

Si el giróstato es cercano a un esfera $(A \approx B \approx C)$, tenemos que

$$
\frac{A+B+C}{2 r^{2}} \approx \frac{3 A}{2 r^{2}}, \quad \frac{3\left(A \cos ^{2} \theta+B \sin ^{2} \theta\right)}{2 r^{2}} \approx \frac{3 A}{2 r^{2}} .
$$


y $\mathcal{V}_{1}(\mathbf{z})=-\frac{1}{r}+\epsilon \mathcal{P}$, donde $\epsilon$ es una cantidad muy pequeña y $\mathcal{P}$ es la perturbación debida a la no-esfericidad del giróstato. Por tanto, el hamiltoniano puede escribirse como

$$
\mathcal{H}_{1}(\mathbf{z})=\frac{1}{2}\left(p_{r}^{2}+\frac{p_{\theta}^{2}}{r^{2}}\right)+\alpha\left(\beta-p_{\theta}\right)^{2}-\frac{1}{r}+\epsilon \mathcal{P} .
$$

Cuando $\epsilon=0$, denotamos de nuevo como $\mathcal{H}$ al nuevo hamiltoniano, así pues,

$$
\mathcal{H}(\mathbf{z})=\frac{1}{2}\left(p_{r}^{2}+\frac{p_{\theta}^{2}}{r^{2}}\right)+\alpha\left(\beta-p_{\theta}\right)^{2}-\frac{1}{r}
$$

Esto describe una aproximación integrable, ya que el hamiltoniano $\mathcal{H}$ y $p_{\theta}$ son dos integrales primeras, independientes y en involución) de la dinámica plana del giróstato en interacción newtoniana con un cuerpo rígido esférico en la fibra del momento angular constante total $\mathbf{M}_{C} \bigcap\left(L_{M_{C}}=L\right)$ en la variedad invariante $\mathbf{M}_{C}$.

\subsection{Potencial ampliado. Regiones de Hill}

\subsubsection{Introducción}

En esta sección vamos a calcular el potencial ampliado y vamos a realizar un estudio de las regiones de Hill, regiones del espacio de configuración donde existe el movimiento real. Dicho potencial se calculará mediante dos métodos. En el primero utilizaremos el lagrangiano asociado a (4.10) y en el segundo usaremos un simple procedimiento algebraico.

\subsubsection{Obtención del potencial ampliado empleando el lagran- giano}

Teorema 50 El lagrangiano asociado a (4.10) es

$$
\mathcal{L}=3\left(\left(\frac{d U_{1}}{d t}\right)^{2}+\left(\frac{d U_{2}}{d t}\right)^{2}\right)+6 \alpha\left(U_{1} \frac{d U_{1}}{d t}+U_{2} \frac{d U_{2}}{d t}\right)+4 \alpha \beta\left(U_{1} \frac{d U_{2}}{d t}-U_{2} \frac{d U_{1}}{d t}\right)+\widetilde{\mathcal{V}}
$$

donde

$$
\widetilde{\mathcal{V}}=\frac{\alpha \beta^{2}}{1+2 \alpha\left(U_{1}^{2}+U_{2}^{2}\right)}-\frac{1}{\sqrt{U_{1}^{2}+U_{2}^{2}}}
$$

con $\tilde{\mathcal{V}}$ el potencial ampliado del sistema.

Para calcular $\mathcal{H}$ usando la transformada de Legendre obtenemos $\mathcal{H}=\mathbf{U}_{\mathbf{1}} \cdot \mathbf{P}-\mathcal{L}$, el cual viene dado por

$$
\mathcal{H}=\frac{|\mathbf{P}|^{2}}{2}+\alpha\left(\beta-\left(U_{1} P_{2}-U_{2} P_{1}\right)\right)^{2}-\frac{1}{\sqrt{U_{1}^{2}+U_{2}^{2}}}
$$

Del lagrangiano (4.11) obtenemos los momentos siguientes

$$
P_{1}=\frac{\partial \mathcal{L}}{\partial \dot{U}_{1}}, \quad P_{2}=\frac{\partial \mathcal{L}}{\partial \dot{U}_{2}}
$$


donde

Finalmente se obtiene

$$
\dot{U}_{1}=\frac{d U_{1}}{d t}, \quad \dot{U}_{2}=\frac{d U_{2}}{d t}
$$

$$
\begin{aligned}
& \frac{d U_{1}}{d t}=P_{1}+2 \alpha\left(\beta-U_{1} P_{2}+U_{2} P_{1}\right) U_{2} \\
& \frac{d U_{2}}{d t}=P_{2}-2 \alpha\left(\beta-U_{1} P_{2}+U_{2} P_{1}\right) U_{1}
\end{aligned}
$$

Entonces usando $\mathbf{U}_{\mathbf{1}} \cdot \mathbf{P}-\mathcal{L}$, obtenemos (4.13).

Las ecuaciones de Lagrange son

$$
\frac{d}{d t}\left(\frac{\partial \mathcal{L}}{\partial \dot{U}_{1}}\right)-\frac{\partial \mathcal{L}}{\partial U_{1}}=0, \quad \frac{d}{d t}\left(\frac{\partial \mathcal{L}}{\partial \dot{U}_{2}}\right)-\frac{\partial \mathcal{L}}{\partial U_{2}}=0
$$

Y puede verificarse ya que $\mathcal{L}=c$, con $c \in \mathbb{R}$.

Observación 51 Obsérvese que $\mathcal{L}=\mathcal{T}+\widetilde{\mathcal{V}}$ donde

$$
\mathcal{T}=3\left(\left(\frac{d U_{1}}{d t}\right)^{2}+\left(\frac{d U_{2}}{d t}\right)^{2}\right)+6 \alpha\left(U_{1} \frac{d U_{1}}{d t}+U_{2} \frac{d U_{2}}{d t}\right)+4 \alpha \beta\left(U_{1} \frac{d U_{2}}{d t}-U_{2} \frac{d U_{1}}{d t}\right)
$$

es la energía cinética generalizada del sistema.

\subsubsection{Obtención del potencial ampliado algebraicamente}

Otra forma de obtener el potencial ampliado es operar en el (4.13) una vez expresado éste en coordenadas polares simplécticas. Y sabemos que el hamiltoniano (4.13) expresado en coordenadas polares simplécticas tiene la siguiente expresión $\mathcal{H}: \mathbf{E} \rightarrow \mathbb{R}$ con

$$
\mathcal{H}=\frac{1}{2}\left(p_{r}^{2}+\frac{p_{\theta}^{2}}{r^{2}}\right)+\alpha\left(\beta-p_{\theta}\right)^{2}-\frac{1}{r}
$$

siendo $\mathbf{E}=\mathbb{R}^{+} \times S^{1} \times \mathbb{R}^{2}$ el espacio de fases del sistema.

Las ecuaciones diferenciales asociadas a $\mathcal{H}$ vienen dadas por

$$
\begin{aligned}
& \frac{d r}{d t}=\frac{d \mathcal{H}}{d p_{r}}, \quad \frac{d \theta}{d t}=\frac{d \mathcal{H}}{d p_{\theta}} \\
& \frac{d p_{r}}{d t}=-\frac{d \mathcal{H}}{d r}, \quad \frac{d p_{\theta}}{d t}=-\frac{d \mathcal{H}}{d \theta}
\end{aligned}
$$

Obsérvese que el hamiltoniano (4.14) y el momento angular $p_{\theta}$ son dos integrales primeras independientes del movimiento en involución. Por tanto, el sistema hamiltoniano (4.15) es integrable.

Completando cuadrados obtenemos

$$
\mathcal{H}=\frac{1}{2}\left(p_{r}^{2}+\frac{1+2 \alpha r^{2}}{r^{2}}\left(p_{\theta}-\frac{2 \alpha \beta r^{2}}{1+2 \alpha r^{2}}\right)^{2}\right)+\tilde{\mathcal{V}}
$$

donde

$$
\widetilde{\mathcal{V}}=\frac{\alpha \beta^{2}}{1+2 \alpha r^{2}}-\frac{1}{r}
$$

es el potencial ampliado expresado en coordenadas polares. 


\subsubsection{Variedades de energía y momento constantes. Regiones de Hill}

Al ser (4.14) integrable, con integrales en involución $\mathcal{H}$ y $p_{\theta}$, podemos definir las siguientes subvariedades del espacio de fases del sistema invariantes por el flujo (4.15).

Definición 52 Llamaremos variedades de energía constante a

$$
\mathcal{H}^{-1}(h)=E_{h}=\left\{\left(r, \theta, p_{r}, p_{\theta}\right) \in \mathbf{E}: \mathcal{H}\left(r, \theta, p_{r}, p_{\theta}\right)=h\right\}, h \in \mathbb{R} .
$$

Llamaremos variedades de momento constante a

$$
J_{k}=\left\{\left(r, \theta, p_{r}, p_{\theta}\right) \in \mathbf{E}: p_{\theta}=k\right\}, k \in \mathbb{R} .
$$

Observación 53 En posteriores estudios cualitativos de la dinámica del sistema consideraremos las subvariedades del espacio de fases $I_{h k}=E_{h} \cap J_{k}$ llamadas variedades de energía-momento constante.

Sea $\pi: \mathbf{E} \longrightarrow \mathbb{R}^{+} \times S^{1}$ la proyección natural del espacio de fases $\mathbf{E}$ en el espacio de configuración $\mathbb{R}^{+} \times S^{1}$.

Definición 54 Para cada $h \in \mathbb{R}$ la región de Hill $R_{h}$ está definida por $R_{h}=\pi\left(E_{h}\right)$.

$$
R_{h}=\left\{(r, \theta) \in \mathbb{R}^{+} \times S^{1}: \widetilde{\mathcal{V}} \leq h\right\}=\left\{(r, \theta) \in \mathbb{R}^{+} \times S^{1}: \frac{\alpha \beta^{2}}{1+2 \alpha r^{2}}-\frac{1}{r} \leq h\right\}
$$

Utilizaremos el símbolo $\approx$ para expresar el difeomorfismo entre dos variedades diferenciales. Es fácil ver que $R_{h} \approx\left\{r \in \mathbb{R}^{+}: \frac{\alpha \beta^{2}}{1+2 \alpha r^{2}}-\frac{1}{r} \leq h\right\} \times S^{1}$.

Definición 55 Las superficies de velocidad nula $S_{h}$ vienen dadas por

$$
S_{h}=\left\{(r, \theta) \in \mathbb{R}^{+} \times S^{1}: \widetilde{\mathcal{V}}=h\right\}=\left\{r \in \mathbb{R}^{+}: \frac{\alpha \beta^{2}}{1+2 \alpha r^{2}}-\frac{1}{r}=h\right\} \times S^{1}
$$

\subsection{Estudio cualitativo del flujo hamiltoniano aso- ciado a $\mathcal{H}$}

\subsubsection{Introducción}

En este capítulo, usaremos técnicas similares a las introducidas en ([36]) para realizar un estudio cualitativo del flujo (4.15). En primer lugar analizaremos topológicamente las regiones de Hill del problema, dando una clasificación de los subconjuntos del espacio de configuración donde existe movimiento real.

Posteriormente, usando el teorema de Liouville-Arnold y métodos analíticos describiremos:

i) La foliación del espacio de fases $\mathbf{E}$ por las variedades de energía $E_{h}$.

ii) La foliación de las variedades de energía $E_{h}$ por las variedades energía-momento $I_{h k}$.

iii) La foliación de $I_{h k}$ por el flujo del sistema hamiltoniano asociado a $\mathcal{H}$. 


\subsubsection{Topología de las regiones de Hill}

Las regiones de Hill estarán completamente caracterizadas por medio de la función potencial (4.16). Son regiones del espacio fásico, donde existe movimiento real y están completamente caracterizadas por medio de los puntos críticos del potencial. Dichos puntos son las raíces reales de la ecuación polinómica

$$
1+4 \alpha r^{2}-4 \alpha^{2} \beta^{2} r^{3}+4 \alpha^{2} r^{4}=0,
$$

que se obtiene derivando $\widetilde{\mathcal{V}}$ respecto de $r$ e igualando a cero.

Discutimos, de acuerdo a los diferentes valores de los parámetros $\alpha$ y $\beta$, el número de raíces reales positivas respecto de $r$ de la ecuación (4.21). Para ello empleamos el método de Sturm, obteniendo el siguiente resultado (véase apéndice A) para la demostración del mismo.

Proposición 56 Cuando $27 \alpha \beta^{4}-128>0$ la ecuación (4.21) tiene dos raíces reales positivas. En el caso en que $27 \alpha \beta^{4}-128=0$ la ecuación (4.21) tiene una raíz real positiva. Y por último $27 \alpha \beta^{4}-128<0$ la ecuación (4.21) no tiene raíces reales.

En lo que sigue los valores del potencial ampliado en cada una de las familias de puntos críticos serán denotados por $h_{i}=\widetilde{\mathcal{V}}\left(r_{i}\right)(i=1,2,3)$, los valores $A_{i}(i=1,2,3)$, serán los puntos de corte de la gráfica del potencial ampliado con $\widetilde{\mathcal{V}}=h$.

Recordemos, por otro lado, que las regiones de Hill, para cada $h \in \mathbb{R}$, estaban definidas de la siguiente forma

$$
R_{h}=\left\{(r, \theta) \in \mathbb{R}^{+} \times S^{1}: \widetilde{\mathcal{V}} \leq h\right\}=\left\{(r, \theta) \in \mathbb{R}^{+} \times S^{1}: \frac{\alpha \beta^{2}}{1+2 \alpha r^{2}}-\frac{1}{r} \leq h\right\}
$$

y que además cumplían que $R_{h} \approx\left\{r \in \mathbb{R}^{+}: \frac{\alpha \beta^{2}}{1+2 \alpha r^{2}}-\frac{1}{r} \leq h\right\} \times S^{1}$, donde el potencial ampliado en coordenadas polares-simplécticas era $\widetilde{\mathcal{V}}=\frac{\alpha \beta^{2}}{1+2 \alpha r^{2}}-\frac{1}{r}$.

Para poder entender la topología de las regiones de Hill introducimos las siguientes figuras:

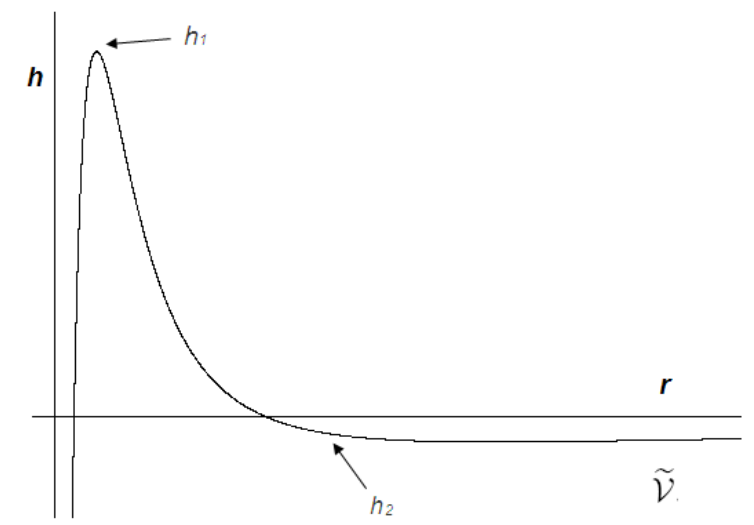

Figura 4.1: Potencial ampliado para $27 \alpha \beta^{4}-128>0$, donde $h_{1}, h_{2}$ son los puntos críticos de $\widetilde{\mathcal{V}}$.

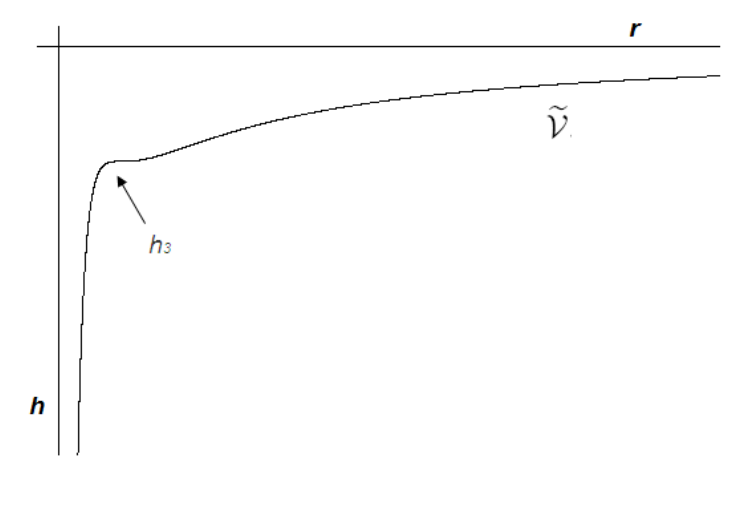

Figura 4.2: Potencial ampliado para $27 \alpha \beta^{4}-128=0$, donde $h_{3}$ es el punto crítico de $\widetilde{\mathcal{V}}$. 


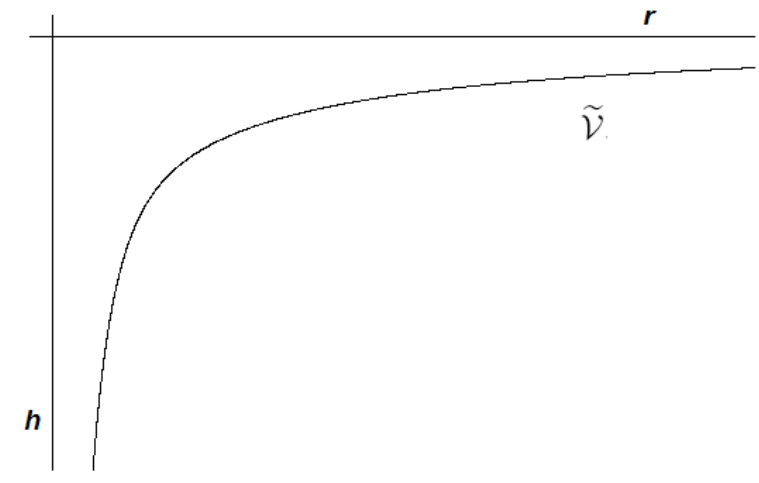

Figura 4.3: Potencial ampliado para $27 \alpha \beta^{4}-128<0$.

Empleando los resultados anteriores se tiene la siguiente clasificación topológica de las regiones de Hill $R_{h}$.

\begin{tabular}{|c|c|c|}
\hline & $h<h_{2}$ & $R_{h} \approx\left(0, A_{1}\right] \times S^{1}$ \\
\hline \multirow{5}{*}{$\begin{array}{c}27 \alpha \beta^{4}-128>0 \\
\text { (véase Figura } 4.1 \text { ) }\end{array}$} & $h=h_{2}$ & $R_{h} \approx\left[\left(0, A_{1}\right] \cup\left\{r_{2}\right\}\right] \times S^{1}$ \\
\hline & $h_{2}<h<0$ & $R_{h} \approx\left[\left(0, A_{1}\right] \cup\left[A_{2}, A_{3}\right]\right] \times S^{1}$ \\
\hline & $0 \leq h<h_{1}$ & $R_{h} \approx\left[\left(0, A_{1}\right] \cup\left[A_{2},+\infty\right)\right] \times S^{1}$ \\
\hline & $h=h_{1}$ & $R_{h} \approx(0,+\infty) \times S^{1}$ \\
\hline & $h_{1}<h$ & $R_{h} \approx(0,+\infty) \times S^{1}$ \\
\hline
\end{tabular}

Tabla 4.1: Clasificación topológica de las regiones de Hill para $27 \alpha \beta^{4}-128>0$. 


\begin{tabular}{|c|c|l|}
\hline & $h<h_{3}$ & $R_{h} \approx\left(0, A_{1}\right] \times S^{1}$ \\
\cline { 2 - 3 } & $h=h_{3}$ & $R_{h} \approx\left(0, r_{3}\right] \times S^{1}$ \\
$\begin{array}{c}27 \alpha \beta^{4}-128=0 \\
\text { véase Figura 4.2) }\end{array}$ & $h_{3}<h<0$ & $R_{h} \approx\left(0, A_{1}\right] \times S^{1}$ \\
\cline { 2 - 3 } & $0 \leq h$ & $R_{h} \approx(0,+\infty) \times S^{1}$ \\
\hline
\end{tabular}

Tabla 4.2: Clasificación topológica de las regiones de Hill para $27 \alpha \beta^{4}-128=0$.

\begin{tabular}{|c|l|l|}
\hline & $h<0$ & $R_{h} \approx\left(0, A_{1}\right] \times S^{1}$ \\
$27 \alpha \beta^{4}-128<0$ & $0 \leq h$ & $R_{h} \approx(0,+\infty) \times S^{1}$ \\
\cline { 2 - 3 }$($ véase Figura 4.3) & 0 & \\
\hline
\end{tabular}

Tabla 4.3: Clasificación topológica de las regiones de Hill para $27 \alpha \beta^{4}-128<0$ 


\subsubsection{Estudio cualitativo del flujo hamiltoniano}

En esta sección estudiamos la topología de las variedades invariantes $\mathcal{H}^{-1}(h)=E_{h} \mathrm{e}$ $I_{h k}$. Con idea de dar una clasificación topológica a dichas variedades necesitamos nuevos resultados e introducir la siguiente notación.

Recordemos que los conjuntos $E_{h}$ e $I_{h k}$ venían dados por

$$
\begin{gathered}
E_{h}=\left\{\left(r, \theta, p_{r}, p_{\theta}\right) \in \mathbf{E}: \mathcal{H}\left(r, \theta, p_{r}, p_{\theta}\right)=h\right\}, h \in \mathbb{R}, \\
J_{k}=\left\{\left(r, \theta, p_{r}, p_{\theta}\right) \in \mathbf{E}: p_{\theta}=k\right\}, k \in \mathbb{R}, \\
I_{h k}=E_{h} \cap J_{k},
\end{gathered}
$$

con $z=\left(r, \theta, p_{r}, p_{\theta}\right) \in \mathbf{E} \mathrm{y}(h, k) \in \mathbb{R}^{2}$.

Definimos ahora una nueva aplicación $g: \mathbb{R}^{+} \times \mathbb{R}^{2} \longrightarrow \mathbb{R}$, con $g\left(r, p_{r}, p_{\theta}\right)=\mathcal{H}\left(r, \theta, p_{r}, p_{\theta}\right)$, es decir

$$
g\left(r, p_{r}, p_{\theta}\right)=\frac{1}{2}\left(p_{r}^{2}+\frac{p_{\theta}^{2}}{r^{2}}\right)+\alpha\left(\beta-p_{\theta}\right)^{2}-\frac{1}{r} .
$$

Si $h \in \mathbb{R}$ es un valor regular de la aplicación $g: \mathbb{R}^{+} \times \mathbb{R}^{2} \longrightarrow \mathbb{R}$ y $g^{-1}(h) \neq \emptyset, g^{-1}(h)$ es una superficie de $\mathbb{R}^{+} \times \mathbb{R}^{2}$ que llamaremos superficie de energía.

Así pues, en términos de la superficie de energía, los conjuntos invariantes quedan definidos de la siguiente manera

$$
\begin{gathered}
E_{h}=\mathcal{H}^{-1}(h)=\left\{\left(r, \theta, p_{r}, p_{\theta}\right) \in \mathbf{E}: g\left(r, p_{r}, p_{\theta}\right)=h\right\} \approx g^{-1}(h) \times S^{1}, \\
J_{k}=\left\{z \in \mathbf{E}: p_{\theta}=k\right\}, k \in \mathbb{R}, \\
I_{h k}=E_{h} \cap J_{k} \approx\left(g^{-1}(h) \cap\left\{p_{\theta}=k\right\}\right) \times S^{1},
\end{gathered}
$$

con $z=\left(r, \theta, p_{r}, p_{\theta}\right) \in \mathbf{E}$ y $(h, k) \in \mathbb{R}^{2}$.

Para el estudio de la topología de $\mathcal{H}^{-1}(h)=E_{h}$ y de $I_{h k},(h, k) \in \mathbb{R}^{2}$ debemos caracterizar primero los puntos de equilibrio del hamiltoniano (4.14), ya que las superficies de energía son regulares cuando $h \neq h_{i}$, siendo $h_{i}=\mathcal{H}\left(z_{e_{i}}\right)$ donde $z_{e_{i}}$ se corresponden con los puntos de equilibrio de $\mathcal{H}$. Y por último debemos calcular los extremos de la superficie de energía $g^{-1}(h)$.

\subsubsection{Puntos de equilibrio de $\mathcal{H}$}

Mediante un cálculo estándar se puede verificar el siguiente resultado:

Lema $57 z_{e}=\left(r_{e}, \theta_{e}, p_{r_{e}}, p_{\theta_{e}}\right) \in \mathbf{E}$ es un equilibrio de las ecuaciones hamiltonianas

$$
\begin{aligned}
\frac{d r}{d t}=\frac{d \mathcal{H}}{d p_{r}}, \quad \frac{d \theta}{d t}= & \frac{d H}{d p_{\theta}} \\
\frac{d p_{r}}{d t} & =-\frac{d H}{d r}, \quad \frac{d p_{\theta}}{d t}=-\frac{d H}{d \theta}
\end{aligned}
$$

si y sólo si $\tilde{z_{e}}=\left(r_{e}, \theta_{e}\right)$ es punto crítico de

$$
\widetilde{\mathcal{V}}=\frac{\alpha \beta^{2}}{1+2 \alpha r^{2}}-\frac{1}{r}
$$

Obsérvese que $\pi\left(\tilde{z}_{e}\right)=z_{e}$, donde $\pi: \mathbf{E} \longrightarrow \mathbb{R}^{+} \times S^{1}$ es la proyección natural. 
Utilizando el algoritmo de Sturm para saber el número de raíces reales de la ecuación

$$
1+4 \alpha r^{2}-4 \alpha^{2} \beta^{2} r^{3}+4 \alpha^{2} r^{4}=0
$$

Obtenemos el siguiente resultado:

Proposición 58 Cuando $27 \alpha \beta^{4}-128>0$ el sistema hamiltoniano (4.15) tiene dos familias de equilibrios. En el caso en que $27 \alpha \beta^{4}-128=0$ el sistema hamiltoniano (4.15) tiene una familia de equilibrios. Y por último, si $27 \alpha \beta^{4}-128<0$ el sistema hamiltoniano (4.15) no tiene equilibrios.

En lo que sigue, los valores de la energía en cada una de las familias de equilibrios serán denotadas por $h_{i}=\mathcal{H}\left(r_{i}, \theta, 0, p_{\theta_{i}}\right), i=1,2,3$.

Observación 59 Por el lema anterior podemos afirmar que los valores $h_{i}=\mathcal{H}\left(r_{i}, \theta, 0, p_{\theta_{i}}\right)$ son los mismos $h_{i}$ utilizados en el estudio de las regiones de Hill.

La proposición anterior caracteriza completamente la topología de $\mathcal{H}^{-1}(h)=E_{h}$.

\subsubsection{Extremos relativos de la superficie de energía $g^{-1}(h)$}

Utilizando el teorema de la Función Implícita para la aplicación $g$, los máximos y mínimos de la superficie $g^{-1}(h)$ vienen dados por las raíces reales de la ecuación polinómica

$$
-1+2\left(\alpha \beta^{2}-h\right) k^{2}-4 \alpha \beta k^{3}+2 \alpha k^{4}=0 .
$$

Utilizando el algoritmo de Sturm podemos calcular el número de raíces reales de (4.23) con respecto a $k=p_{\theta}$. Dichas raíces son $c_{j}(j=1,2,3,4)$ y corresponden a los extremos de la superficie de energía $Q_{j}(j=1,2,3,4)$. El cálculo de dichos extremos podemos encontrarlo en el apéndice B.

Al aplicar el algortitmo de Sturm a (4.23) nos encontramos con la siguiente ecuación (véase apéndice $\mathrm{C}$ )

$$
h-\alpha(\beta-k)^{2}=0
$$

cuyas raiíces reales respecto de $k$ son $a_{1}$ y $a_{2}$. La importancia de estos dos valores y de esta ecuación es que entre $a_{1}$ y $a_{2}$, todas las trayectorias no son acotadas. En el caso de que $k=0$ las órbitas tampoco son acotadas. Por último, en el resto de los casos o no tendremos superficie de energía o tendremos un punto o trayectorias acotadas.

Según los diferentes niveles de energía tenemos que 


\begin{tabular}{|c|c|c|}
\hline \multirow{6}{*}{$27 \alpha \beta^{4}-128>0$} & $h \leq h_{2}$ & $\begin{array}{l}\text { Máximo }=c_{1} \\
\text { Mínimo }=c_{2}\end{array}$ \\
\hline & $h=h_{2}$ & $\begin{array}{l}\text { Máximo }=c_{1} \\
\text { Mínimo }=c_{2}\end{array}$ \\
\hline & $h_{2}<h<0$ & $\begin{array}{l}\text { Máximo }=c_{1} \\
\text { Mínimo }=c_{2} \\
\text { Máximo }=c_{4} \\
\text { Mínimo }=c_{3}\end{array}$ \\
\hline & $0<h<h_{1}$ & $\begin{array}{l}\text { Máximo }=c_{1} \\
\text { Mínimo }=c_{2} \\
\text { Máximo }=c_{4} \\
\text { Mínimo }=c_{3}\end{array}$ \\
\hline & $h=h_{1}$ & $\begin{array}{l}\text { Máximo }=c_{1} \\
\text { Mínimo }=c_{2}\end{array}$ \\
\hline & $h_{1} \leq h$ & $\begin{array}{l}\text { Máximo }=c_{1} \\
\text { Mínimo }=c_{2}\end{array}$ \\
\hline
\end{tabular}

Tabla 4.4: Extremos relativos de la superficie de energía $g^{-1}$ para $27 \alpha \beta^{4}-128>0$ 


\begin{tabular}{|l|l|l|}
\hline & & Máximo $=c_{1}$ \\
$27 \alpha \beta^{4}-128=0$ & Mínimo $=c_{2}$ \\
\cline { 2 - 3 } & $h=h_{3}$ & Máximo $=c_{1}$ \\
& Mínimo $=c_{2}$ \\
\cline { 2 - 3 } & $h_{3}<h<0$ & Máximo $=c_{1}$ \\
& Mínimo $=c_{2}$ \\
\hline
\end{tabular}

Tabla 4.5: Extremos relativos de la superficie de energía $g^{-1}$ para $27 \alpha \beta^{4}-128=0$

\begin{tabular}{|l|l|l|}
\hline \multirow{1}{*}{$27 \alpha \beta^{4}-128<0$} & $0<h$ & Máximo $=c_{1}$ \\
& & Mínimo $=c_{2}$ \\
\cline { 2 - 3 } & $h<0$ & Máximo $=c_{1}$ \\
& & Mínimo $=c_{2}$ \\
\cline { 2 - 3 } & $h \geq 0$ & Máximo $=c_{1}$ \\
& Mínimo $=c_{2}$ \\
\hline
\end{tabular}

Tabla 4.6: Extremos relativos de la superficie de energía $g^{-1}$ para $27 \alpha \beta^{4}-128<0$ 


\subsubsection{Clasificación topológica de $E_{h}$ e $I_{h k}$}

En esta parte del capítulo estudiaremos la topología de las variedades invariantes $\mathcal{H}^{-1}(h)=E_{h}$ e $I_{h k}$. Para ello introduzcamos de nuevo toda aquella notación que es necesaria para entender las distintas topologías.

Denotamos por $h_{i}=\mathcal{H}\left(r_{i}, \theta_{i}, 0, p_{\theta_{i}}\right)(i=1,2,3)$, los valores del hamiltoniano $\mathcal{H}$ en cada uno de sus puntos de equilibrio. La aplicación $g: \mathbb{R}^{+} \times \mathbb{R}^{2} \longrightarrow \mathbb{R}$ está definida por $g\left(r, p_{r}, p_{\theta}\right)=\mathcal{H}\left(r, \theta, p_{r}, p_{\theta}\right)$, y en el caso de que $h \in \mathbb{R}$ sea un valor regular para $g$ y $g^{-1}(h) \neq \emptyset$, entonces $g^{-1}(h)$ es una superficie de $\mathbb{R}^{+} \times \mathbb{R}^{2}$ llamada superficie de energía, además $g^{-1}(h)=E_{h} / S^{1}$. Los valores de $k$ que son raíces de la ecuación (4.23) los denotamos por $c_{j}(j=1,2,3,4)$ y corresponden a los extremos $Q_{j}(j=1,2,3,4)$ de la superficie de energía.

Los valores $a_{1}$ y $a_{2}$ son las raíces reales respecto de $k$ de la ecuación $h-\alpha(\beta-k)^{2}=0$, y nos delimitan aquellas regiones en las que están o no acotadas las óbitas. Recordemos que cuando $k=0$ las órbitas no están acotadas.

Finalmente, $S^{n-1}$ es la esfera en $\mathbb{R}^{n}$, con $n>1$ e $Y$ es la unión de dos toros sólidos abiertos que se identifican punto a punto con los puntos de dos círculos de cada toro, que no pueden ser contraidos a un solo punto dentro del correspondiente toro (véase [36] para más detalles).

Los diferentes casos se muestran a través de las siguientes figuras y de sus correspondientes tablas.

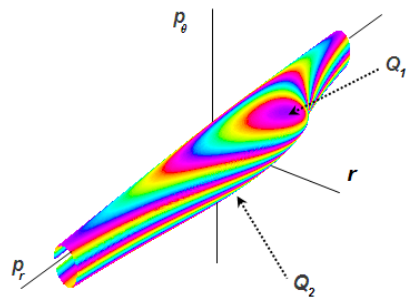

Figura 4.4: $\quad \mathcal{H}^{-1}(h) / S^{1}$ para $27 \alpha \beta^{4}-128>0$ y $h<h_{2}$, donde $k=p_{\theta}$ y $Q_{1}, Q_{2}$ los extremos.

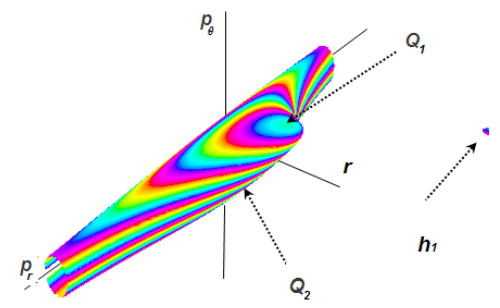

Figura 4.5: $\quad \mathcal{H}^{-1}(h) / S^{1}$ para $27 \alpha \beta^{4}-128>0$ y $h=h_{2}$, donde $k=p_{\theta}, h_{2}$ el punto de equilibrio y $Q_{1}, Q_{2}$ los extremos. 


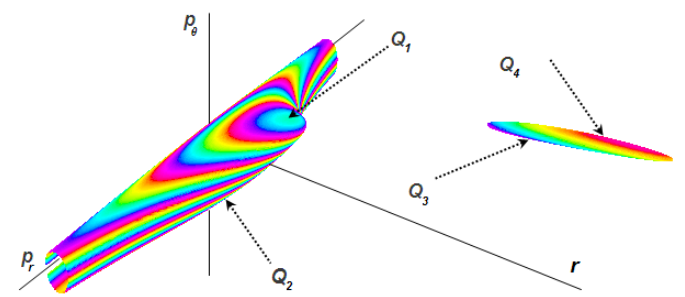

Figura 4.6: $\mathcal{H}^{-1}(h) / S^{1}$ para $27 \alpha \beta^{4}-128>0$ y $h_{2}<h<0$, donde $k=p_{\theta}$ y $Q_{1}, Q_{2}, Q_{3}, Q_{4}$ los extremos.

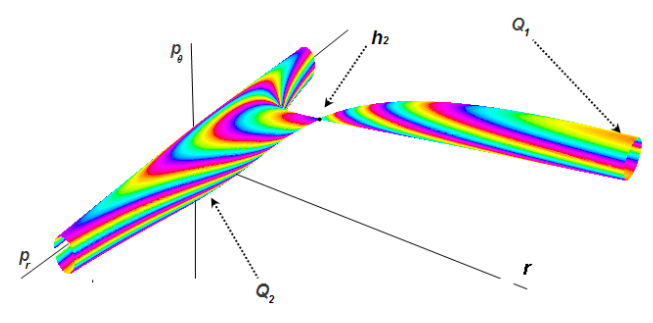

Figura 4.8: $\quad \mathcal{H}^{-1}(h) / S^{1}$ para $27 \alpha \beta^{4}-128>0$ y $h=h_{1}$, donde $k=p_{\theta}, h_{1}$ el punto de equilibrio y $Q_{1}, Q_{2}$ los extremos.

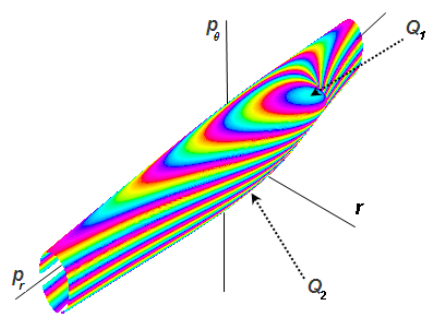

Figura 4.10: $\mathcal{H}^{-1}(h) / S^{1}$ para $27 \alpha \beta^{4}-128=0$ y $h<h_{3}$, donde $k=p_{\theta}$ y $Q_{1}, Q_{2}$ los extremos.

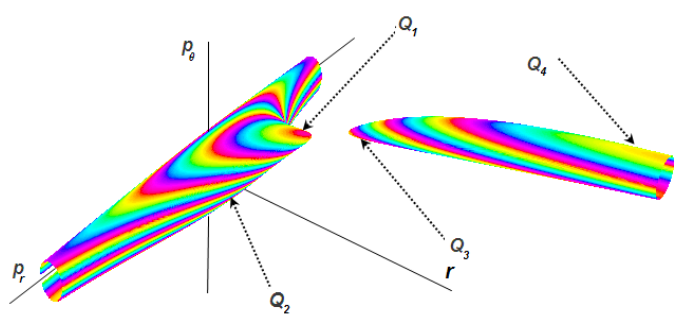

Figura 4.7: $\quad \mathcal{H}^{-1}(h) / S^{1} \quad$ para $27 \alpha \beta^{4}-128>0$ y $0<h<h_{1}$, donde $k=p_{\theta}$ y $Q_{1}, Q_{2}, Q_{3}, Q_{4}$ los extremos.

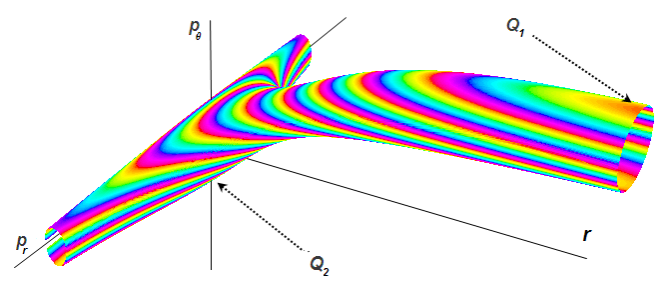

Figura 4.9: $\mathcal{H}^{-1}(h) / S^{1}$ para $27 \alpha \beta^{4}-128>0$ y $h>h_{1}$, donde $k=p_{\theta}$ y $Q_{1}, Q_{2}$ los extremos.

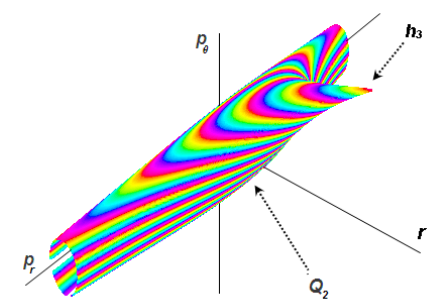

Figura 4.11: $\mathcal{H}^{-1}(h) / S^{1}$ para $27 \alpha \beta^{4}-128=0$ y $h=h_{3}$, donde $k=p_{\theta}, h_{3}$ el punto de equilibrio $\mathrm{y}$ $Q_{2}$ los extremos. 


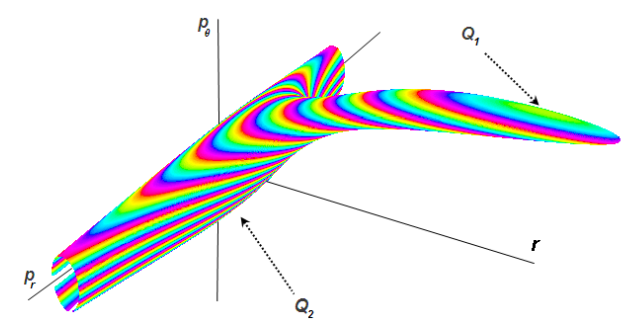

Figura 4.12: $\mathcal{H}^{-1}(h) / S^{1}$ para $27 \alpha \beta^{4}-128=0$ y $h_{3}<h<0$, donde $k=p_{\theta}$ у $Q_{1}$ у $Q_{2}$ los extremos.

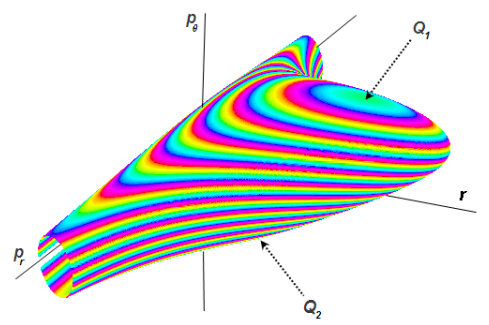

Figura 4.14: $\quad \mathcal{H}^{-1}(h) / S^{1}$ para $27 \alpha \beta^{4}-128<0$ y $h<0$, donde $k=p_{\theta}$ y $Q_{1}, Q_{2}$ los extremos.

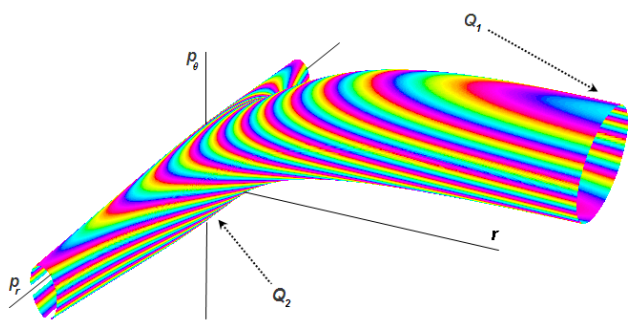

Figura 4.13: $\mathcal{H}^{-1}(h) / S^{1}$ para $27 \alpha \beta^{4}-128=0$ y $0<h$, donde $k=p_{\theta}$ у $Q_{1}$ y $Q_{2}$ los extremos.

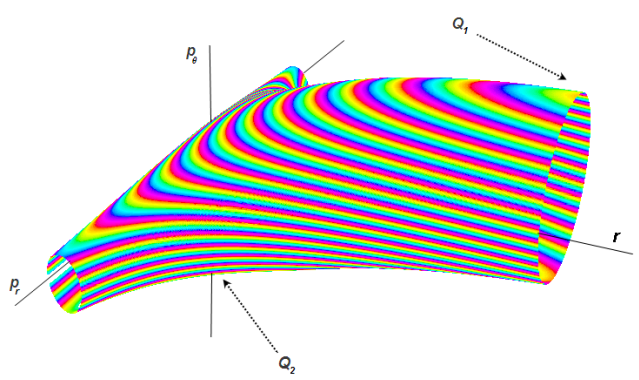

Figura 4.15: $\quad \mathcal{H}^{-1}(h) / S^{1}$ para $27 \alpha \beta^{4}-128<0$ y $h \geq 0$, donde $k=p_{\theta}$ y $Q_{1}, Q_{2}$ los extremos.

\begin{tabular}{|c|c|ll|}
\hline \multicolumn{1}{|c|}{$E_{h}$} & & \multicolumn{1}{c|}{$I_{h k}$} \\
\hline \multirow{5}{*}{$h<h_{2}$} & \multirow{4}{*}{$S^{2} \backslash S^{0}$}, véase 4.4 & $\emptyset$ & $k<c_{2}$ \\
& & $S^{1}$ & $k=c_{2}$ \\
& & $S^{1} \times S^{1}$ & $c_{2}<k<0$ \\
& & $S^{1} \times \mathbb{R}$ & $k=0$ \\
& & $S^{1} \times S^{1}$ & $0<k<c_{1}$ \\
& & $S^{1}$ & $k=c_{1}$ \\
& & $\emptyset$ & $c_{1}<k$ \\
& & $\emptyset$ & $k<c_{2}$ \\
& & $S^{1}$ & $k=c_{2}$ \\
& $\multirow{5}{*}{S^{2} \backslash S^{0}} \cup\left\{S^{0}\right\}$, véase 4.5 & $S^{1} \times S^{1}$ & $c_{2}<k<0$ \\
& & $S^{1} \times \mathbb{R}$ & $k=0$ \\
& & $S^{1}$ & $0<k<c_{1}$ \\
& & $\emptyset$ & $k=c_{1}$ \\
& & $S^{1}$ & $c_{1}<k<h_{2}$ \\
& & $\emptyset$ & $k=h_{2}$ \\
& & $\emptyset$ & $h_{2}<k$ \\
& & $S^{1}$ & $k<c_{2}$ \\
& & $S^{1} \times S^{1}$ & $c_{2}<k<0$
\end{tabular}

(Sigue en la página siguiente) 


\begin{tabular}{|c|c|c|c|}
\hline$h$ & $E_{h}$ & & $I_{h k}$ \\
\hline$h_{2}<h<0$ & $\left\{S^{2} \backslash S^{0}\right\} \cup\left\{S^{2}\right\}$, véase 4.6 & $\begin{array}{l}S^{1} \times \mathbb{R} \\
S^{1} \times S^{1} \\
S^{1} \\
\emptyset \\
S^{1} \\
S^{1} \times S^{1} \\
S^{1} \\
\emptyset\end{array}$ & $\begin{array}{l}k=0 \\
0<k<c_{1} \\
k=c_{1} \\
c_{1}<k<c_{3} \\
k=c_{3} \\
c_{3}<k<c_{4} \\
k=c_{4} \\
c_{4}<k\end{array}$ \\
\hline $0<h<h_{1}$ & $\left\{S^{2} \backslash S^{0}\right\} \cup\left\{S^{2} \backslash S^{0}\right\}$, véase 4.7 & $\begin{array}{l}\emptyset \\
S^{1} \\
S^{1} \times S^{1} \\
S^{1} \times \mathbb{R} \\
S^{1} \times S^{1} \\
S^{1} \\
\emptyset \\
S^{1} \\
S^{1} \times S^{1} \\
S^{1} \times \mathbb{R} \\
S^{1} \times S^{1} \\
S^{1} \\
\emptyset \\
\end{array}$ & $\begin{array}{l}k<c_{2} \\
k=c_{2} \\
c_{2}<k<0 \\
k=0 \\
0<k<c_{1} \\
k=c_{1} \\
c_{1}<k<c_{3} \\
k=c_{3} \\
c_{3}<k<a_{1} \\
a_{1} \leq k \leq a_{2} \\
a_{2}<k<c_{4} \\
k=c_{4} \\
c_{4}<k\end{array}$ \\
\hline$h=h_{1}$ & $Y$, véase 4.8 & $\begin{array}{l}\emptyset \\
S^{1} \\
S^{1} \times S^{1} \\
S^{1} \times \mathbb{R} \\
S^{1} \times S^{1} \\
S^{1} \times \mathbb{R} \\
S^{1} \times S^{1} \\
S^{1} \\
\emptyset\end{array}$ & $\begin{array}{l}k<c_{2} \\
k=c_{2} \\
c_{2}<k<0 \\
k=0 \\
0<k<a_{1} \\
a_{1} \leq k \leq a_{2} \\
a_{2}<k<c_{1} \\
k=c_{1} \\
c_{1}<k\end{array}$ \\
\hline$h>h_{1}$ & $S^{2} \backslash\left\{S^{0} \cup S^{0}\right\}$, véase 4.9 & $\begin{array}{l}\emptyset \\
S^{1} \\
S^{1} \times S^{1} \\
S^{1} \times \mathbb{R} \\
S^{1} \times S^{1} \\
S^{1} \times \mathbb{R} \\
S^{1} \times S^{1} \\
S^{1} \\
\emptyset\end{array}$ & $\begin{array}{l}k<c_{2} \\
k=c_{2} \\
c_{2}<k<0 \\
k=0 \\
0<k<a_{1} \\
a_{1} \leq k \leq a_{2} \\
a_{2}<k<c_{1} \\
k=c_{1} \\
c_{1}<k\end{array}$ \\
\hline
\end{tabular}

Tabla 4.7: Clasificación topológica de $E_{h}$ y $I_{h k}$ cuando $27 \alpha \beta^{4}-128>0$. 


\begin{tabular}{|c|c|c|c|}
\hline$h$ & $E_{h}$ & & $I_{h k}$ \\
\hline$h<h_{3}$ & $S^{2} \backslash S^{0}$, véase 4.10 & $\begin{array}{l}\emptyset \\
S^{1} \\
S^{1} \times S^{1} \\
S^{1} \times \mathbb{R} \\
S^{1} \times S^{1} \\
S^{1} \\
\emptyset\end{array}$ & $\begin{array}{l}k<c_{2} \\
k=c_{2} \\
c_{2}<k<0 \\
k=0 \\
0<k<c_{1} \\
k=c_{1} \\
c_{1}<k\end{array}$ \\
\hline$h=h_{3}$ & $S^{2} \backslash S^{0}$, véase 4.11 & $\begin{array}{l}\emptyset \\
S^{1} \\
S^{1} \times S^{1} \\
S^{1} \times \mathbb{R} \\
S^{1} \times S^{1} \\
S^{1} \\
\emptyset\end{array}$ & $\begin{array}{l}k<c_{2} \\
k=c_{2} \\
c_{2}<k<0 \\
k=0 \\
0<k<c_{1} \\
k=c_{1} \\
c_{1}<k\end{array}$ \\
\hline$h_{3}<h<0$ & $S^{2} \backslash S^{0}$, véase 4.12 & $\begin{array}{l}\emptyset \\
S^{1} \\
S^{1} \times S^{1} \\
S^{1} \times \mathbb{R} \\
S^{1} \times S^{1} \\
S^{1} \\
\emptyset\end{array}$ & $\begin{array}{l}k<c_{2} \\
k=c_{2} \\
c_{2}<k<0 \\
k=0 \\
0<k<c_{1} \\
k=c_{1} \\
c_{1}<k\end{array}$ \\
\hline $0<h$ & $S^{2} \backslash\left\{S^{0} \cup S^{0}\right\}$, véase 4.13 & $\begin{array}{l}\emptyset \\
S^{1} \\
S^{1} \times S^{1} \\
S^{1} \times \mathbb{R} \\
S^{1} \times S^{1} \\
S^{1} \times \mathbb{R} \\
S^{1} \times S^{1} \\
S^{1} \\
\emptyset\end{array}$ & $\begin{array}{l}k<c_{2} \\
k=c_{2} \\
c_{2}<k<0 \\
k=0 \\
0<k<a_{1} \\
a_{1} \leq k \leq a_{2} \\
a_{2}<k<c_{1} \\
k=c_{1} \\
c_{1}<k\end{array}$ \\
\hline
\end{tabular}

Tabla 4.8: Clasificación topológica de $E_{h}$ y $I_{h k}$ cuando $27 \alpha \beta^{4}-128=0$. 


\begin{tabular}{|c|c|c|c|}
\hline$h$ & $E_{h}$ & & $I_{h k}$ \\
\hline$h<0$ & $S^{2} \backslash S^{0}$, veáse 4.14 & $\begin{array}{l}\emptyset \\
S^{1} \\
S^{1} \times S^{1} \\
S^{1} \times \mathbb{R} \\
S^{1} \times S^{1} \\
S^{1} \\
\emptyset\end{array}$ & $\begin{array}{l}k<c_{2} \\
k=c_{2} \\
c_{2}<k<0 \\
k=0 \\
0<k<c_{1} \\
k=c_{1} \\
c_{1}<k\end{array}$ \\
\hline$h \geq 0$ & $S^{2} \backslash\left\{S^{0} \cup S^{0}\right\}$, véase 4.15 & $\begin{array}{l}\emptyset \\
S^{1} \\
S^{1} \times S^{1} \\
S^{1} \times \mathbb{R} \\
S^{1} \times S^{1} \\
S^{1} \times \mathbb{R} \\
S^{1} \times S^{1} \\
S^{1} \\
\emptyset\end{array}$ & $\begin{array}{l}k<c_{2} \\
k=c_{2} \\
c_{2}<k<0 \\
k=0 \\
0<k<a_{1} \\
a_{1} \leq k \leq a_{2} \\
a_{2}<k<c_{1} \\
k=c_{1} \\
c_{1}<k\end{array}$ \\
\hline
\end{tabular}

Tabla 4.9: Clasificación topológica de $E_{h}$ e $I_{h k}$ cuando $27 \alpha \beta^{4}-128<0$.

\subsection{Variables acción-ángulo}

En esta sección vamos a calcular, por medio de la teoría de Hamilton-Jacobi , las variables acción-ángulo y la expresión del hamiltoniano en dichas variables. Estas variables son muy útiles para calcular los elementos keplerianos modificados y las ecuaciones planetarias que se derivan de dichos elementos.

Recordemos que nuestro hamiltoniano es

$$
\mathcal{H}=\frac{1}{2}\left(p_{r}^{2}+\frac{p_{\theta}^{2}}{r^{2}}\right)+\alpha\left(\beta-p_{\theta}\right)^{2}-\frac{1}{r}
$$

y que completando cuadrados obtenemos el potencial ampliado escribiendo así el hamiltoniano como ya sabemos

$$
\mathcal{H}=\frac{1}{2}\left(p_{r}^{2}+\frac{1+2 \alpha r^{2}}{r^{2}}\left(p_{\theta}-\frac{2 \alpha \beta r^{2}}{1+2 \alpha r^{2}}\right)^{2}\right)+\widetilde{\mathcal{V}}(r)
$$

siendo $\widetilde{\mathcal{V}}(r)=\frac{\alpha \beta^{2}}{1+2 \alpha r^{2}}-\frac{1}{r}$. 
Como sabemos, $\theta$ es una variable ignorable de $\mathcal{H}$, por lo que podemos definir la siguiente función

$$
W=W_{r}(r)+k \theta
$$

con $\frac{\partial W}{\partial r}=p_{r} \mathrm{y} \frac{\partial W}{\partial \theta}=p_{\theta}=$ cte. Luego la ecuación de hamilton queda como

$$
\left(\frac{\partial W_{r}}{\partial r}\right)^{2}+\frac{1+2 \alpha r^{2}}{r^{2}}\left(k-\frac{2 \alpha \beta r^{2}}{1+2 \alpha r^{2}}\right)^{2}+2 \widetilde{\mathcal{V}}(r)=2 h
$$

donde $h=$ cte (energía del sistema).

Despejando $\frac{\partial W_{r}}{\partial r}$, se tiene que

$$
\frac{\partial W_{r}}{\partial r}=\sqrt{2 h-\frac{1+2 \alpha r^{2}}{r^{2}}\left(k-\frac{2 \alpha \beta r^{2}}{1+2 \alpha r^{2}}\right)^{2}-2 \widetilde{\mathcal{V}}(r)}
$$

Reordenando

$$
\frac{\partial W_{r}}{\partial r}=\sqrt{2 h-2 \widetilde{\mathcal{V}}(r)-\frac{1+2 \alpha r^{2}}{r^{2}}\left(k-\frac{2 \alpha \beta r^{2}}{1+2 \alpha r^{2}}\right)^{2}}
$$

e integrando obtenemos $W_{r}$

$$
W_{r}=\int \sqrt{2 h-2 \widetilde{\mathcal{V}}(r)-\frac{1+2 \alpha r^{2}}{r^{2}}\left(k-\frac{2 \alpha \beta r^{2}}{1+2 \alpha r^{2}}\right)^{2}} d r
$$

Así pues, las variables acción-ángulo está definidas por

$$
\begin{gathered}
J_{\theta}=\frac{1}{2 \pi} \int_{0}^{2 \pi} p_{\theta} d \theta=k \\
J_{r}=\frac{1}{2 \pi} \int_{r_{1}}^{r_{2}} \sqrt{2 h-2 \tilde{\mathcal{V}}(r)-\frac{1+2 \alpha r^{2}}{r^{2}}\left(k-\frac{2 \alpha \beta r^{2}}{1+2 \alpha r^{2}}\right)^{2}} d r
\end{gathered}
$$

donde

$$
\widetilde{\mathcal{V}}(r)=\frac{\alpha \beta^{2}}{1+2 \alpha r^{2}}-\frac{1}{r}
$$

es la expresión del potencial ampliado en coordenadas polares simplécticas y $r_{1}$ y $r_{2}$ son raíces reales distintas de una ecuación que estudiaremos más adelante. 
Para calcular $J_{r}$ se ha usado el teorema de los residuos de Cauchy.

Las expresiones para $J_{\theta}$ y $J_{r}$ son

$$
\begin{gathered}
J_{\theta}=k \\
J_{r}=-J_{\theta}-\frac{1}{\sqrt{2\left(\alpha \beta^{2}-h-2 \alpha \beta J_{\theta}+\alpha J_{\theta}^{2}\right)}}
\end{gathered}
$$

La transformación de variables en polares-simplécticas a variables acción-ángulo se define en la siguiente región mediante la ecuación en $r$

$$
2 h-2 \widetilde{\mathcal{V}}(r)-\frac{1+2 \alpha r^{2}}{r^{2}}\left(k-\frac{2 \alpha \beta r^{2}}{1+2 \alpha r^{2}}\right)^{2}=0
$$

que tiene dos raíces reales diferentes respecto a los parámetros $\alpha, \beta$ y $h\left(r_{1}\right.$ y $\left.r_{2}\right)$.

Por medio del algoritmo de Sturm se obtiene la región donde (5.6) tiene dos raíces reales distintas. Esta región viene dada por las siguientes inecuaciones

$$
\frac{1}{2\left(h-\alpha(\beta-k)^{2}\right)}+k^{2}<0
$$

$$
h-\alpha(\beta-k)^{2}<0
$$


Y la región es

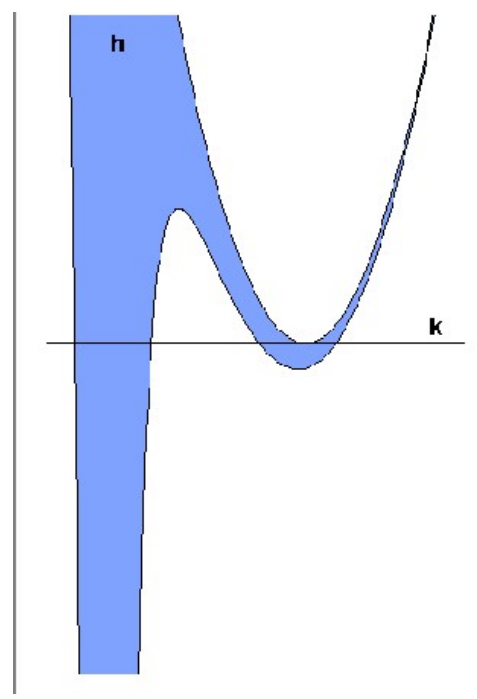

Figura 4.16: Región donde (4.28) tiene dos raíces reales distintas

Resolviendo $h$ en (4.27) tenemos el hamiltoniano en variables acción-ángulo, que se expresa en estas nuevas variables como

$$
\mathcal{H}=-\frac{1}{2\left(J_{r}+J_{\theta}\right)^{2}}+\alpha\left(\beta-J_{\theta}\right)^{2}
$$

\subsection{Variables de Delaunay}

Usando las siguientes transformaciones a variables Delaunay

$$
G=J_{\theta}, \quad L=J_{r}+J_{\theta}
$$

podemos expresar $\mathcal{H}$ como

$$
\mathcal{H}=-\frac{1}{2 L^{2}}+\alpha(\beta-G)^{2}
$$

En variables Delaunay, la región anterior viene dada por las siguientes desigualdades

$$
\begin{gathered}
G^{2}-L^{2}<0 \\
-\frac{1}{2 L^{2}}<0
\end{gathered}
$$


Donde la región es

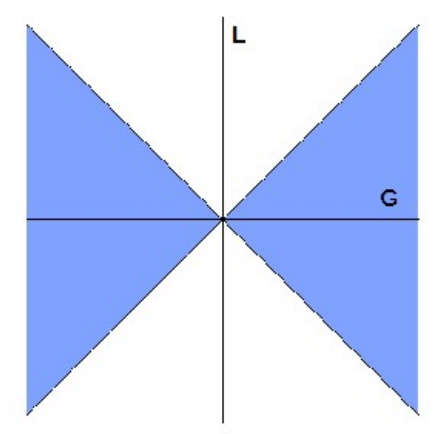

Figura 4.17: Región para las variables de Delaunay

\subsection{Clasificación de órbitas}

En esta sección daremos una clasificación de las órbitas para nuestro problema, en particular estudiaremos las que corresponden a la figura (4.8). Utilizando el Mathematica se ha desarrollado un código para poder dibujarlas (véase apéndice D para más detalles).

Para hacer esta clasificación, tomaremos el hamiltoniano escrito en variables acciónángulo $\left(J_{r}, J_{\theta}\right)$. Cuya expresión es

$$
\mathcal{H}=-\frac{1}{2\left(J_{r}+J_{\theta}\right)^{2}}+\alpha\left(\beta-J_{\theta}\right)^{2}
$$

Encontramos diferentes tipos de órbitas: circular, periódica, cuasiperiódica, de colisión y de escape.

Las circulares aparecen cuando nos situamos en un máximo o en un mínimo de la superficie de energía. Cuando estamos en un punto de equilibrio tendremos como órbita un punto, que corresponde con él mismo. Por otro lado, cuando el cociente $\frac{\partial \mathcal{H}}{\partial J_{r}} / \frac{\partial \mathcal{H}}{\partial J_{\theta}}$ es un número racional $\frac{p}{q}$, obtenemos órbitas periódicas. Finalmente, cuando $k=0$ serán órbitas de colisión y cuando estemos entre $a_{1}$ y $a_{2}$ tendremos órbitas de escape.

Veamos algunos ejemplos de estas órbitas. 


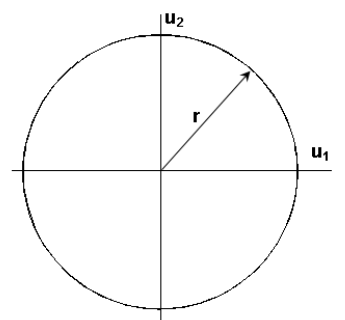

Figura 4.18: Ejemplo de una órbita circular en coordenadas cartesianas $\left(u_{1}, u_{2}\right)$ para la figura 4.8. Estas órbitas aparecen en los extremos de la superficie $m_{i}, i=1,2$.

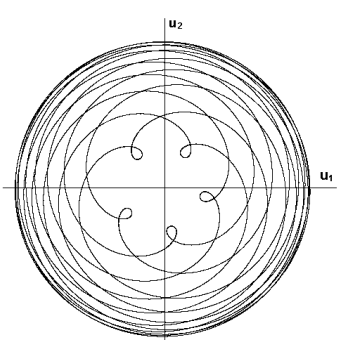

Figura 4.19: Ejemplo de una órbita periódica en coordenadas cartesianas $\left(u_{1}, u_{2}\right)$ para la figura 4.8 .

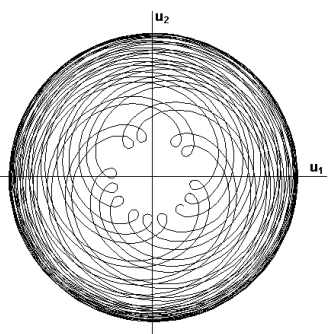

Figura 4.20: Ejemplo de una órbita cuasiperiódica en coordenadas cartesianas $\left(u_{1}, u_{2}\right)$ para la figura 4.8.

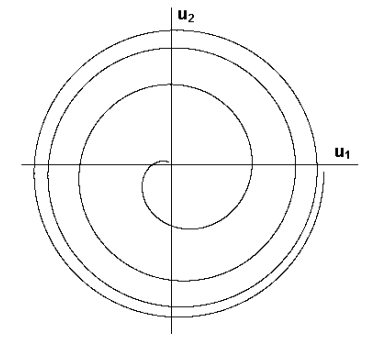

Figura 4.21: Ejemplo de una órbita de colisión en coordenadas cartesianas $\left(u_{1}, u_{2}\right)$ para la figura 4.8. Esta órbita ocurre cuando $k=0$.

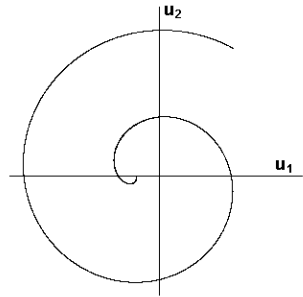

Figura 4.22: Ejemplo de una órbita de escape en coordenadas cartesianas $\left(u_{1}, u_{2}\right)$ para la figura 4.8. Estas aparecen entre los valores $a_{1}$ y $a_{2}$ de la superficeie. 


\subsection{Elemento keplerianos modificados}

Utilizando la teoría de Hamilton-Jacobi podemos obtener las expresiones de los elementos keplerianos modificados, que seguimos llamando $(a, e, l, g)$.

- $a$ es el semieje mayor y su expresión es

$$
a=\frac{-1}{2\left(h-\alpha(\beta-k)^{2}\right)}
$$

- e es la excentricidad

$$
e=\left(1+2\left(h-\alpha(\beta-k)^{2}\right) k^{2}\right)^{\frac{1}{2}}
$$

- l es la anomalía media

$$
l=t-n^{-1}(E-e \sin E)
$$

donde $t$ es el tiempo, $n=a^{-\frac{3}{2}}$ y $E$ es la anomalía excéntrica.

- $g$ es el argumento del perihelio

$$
g=\theta-f-2 \alpha(t-l)\left(a^{\frac{1}{2}}\left(1-e^{2}\right)^{\frac{1}{2}}-\beta\right)
$$

donde $f$ es la anomalía verdadera.

Se puede comprobar que los elementos keplerianos modificados coinciden con los del problema de Kepler cuando $\alpha$ tiende a cero. Se podría realizar una teoría de perturbaciones para este sistema mucho más complicada que la teoría de perturbaciones del problema kepleriano. Para más detalles sobre las ecuaciones planetarias del problema de Kepler véase Goldstein (1988) [31]. 


\subsection{Conclusiones}

En este capítulo se ha considerado una primera aproximación integrable para un problema de tipo roto-traslatorio del movimiento plano de un giróstato en interacción newtoniana con un sólido rígido esférico. Para ello, hemos hecho el estudio en las fibras de valor constante del momento angular total $\left(L_{M_{C}}=L\right)$ en la variedad invariante $\mathbf{M}_{C}$. Se ha descrito la dinámica hamiltoniana en dicha variedad de las superficies $E_{h}, I_{k}$ y $I_{h k}$, por medio del teorema de Liouville-Arnold y otras técnicas específicas. También se ha obtenido una clasificación topológica completa para el flujo fásico asociado al sistema. Las variables acción-ángulo y la región del espacio fásico han sido obtenidas, así como nuestro hamiltoniano expresado en dichas variables. De la misma manera se ha procedido a calcular las variables de Delaunay y el hamiltoniano correspondiente, útiles para poder obtener los elementos keplerianos modificados que servirán para la elaboración de una teoría de perturbaciones. Por último, se ha hecho un estudio de las órbitas en un caso particular y se han calculado las ecuaciones planetarias. Los resultados obtenidos, tienen una aplicación directa al estudio de dos cuerpos de tipo roto-traslatorios, en particular al caso de asteroides binarios, donde la rotación de uno de ellos influye fuertemente en el movimiento orbital del sistema, el otro debe ser una masa puntual o un cuerpo esférico y ambos deben estar a una distancia mucho mayor que sus propias dimensiones. Un ejemplo de este caso es la situación de 45 Eugenia y su luna Petit-Prince (Marchis et al (2004) [42]). 

5

\section{Un sistema Manev en referencia rotante}

\subsection{Introducción}

Una de las primeras y más conocidas afirmaciones de la teoría de la relatividad de Einstein fue las explicación cualitativa correcta del movimiento del perihelio de Mercurio. Sabemos por las leyes de Kepler que todos los planetas describen órbitas elípticas alrededor del Sol, algunas de las cuales son prácticamente circulares. En el caso de Mercurio debido a la gran excentricidad de su órbita, lo que ocurre es que ésta elipse es muy pronunciada. La perturbación orignada por el resto de los planetas del Sistema Solar hace que el perihelio, punto de la órbita del planeta que se encuentra más cercano al Sol, se mueva unos 43 segundos de arco por siglo. Este hecho no se explicaba mediante las leyes de Newton ni de la Mecánica Clásica. Lo que se pretende en este capítulo es dar un modo alternativo de estudiar la precesión de Mercurio usando Mecánica Clásica en lugar de Mecánica Relativista.

Entre 1924 y 1930, G. Manev (véase [38], [39], [40], [41]) estudió la corrección al potencial atractivo newtoniano del tipo $\mathcal{V}=-\gamma / r-\varepsilon /\left(r^{2}\right)$, donde $\gamma=G\left(m_{1}, m_{2}\right)$ ( $G$ es la constante gravitatoria, $m_{1}$ y $m_{2}$ las masas de dos cuerpos que se atraen mutuamente y $r$ la distancia que hay entre ellos).

En las últimas décadas algunos autores han estudiado este tipo de sistemas. A. V. Bobylev et al (1997) [15] consideran la ley gravitacional de Manev en el contexto de la dinámica estelar.

Consideremos ahora el objeto de nuestro estudio. En algunas variedades invariantes del movimiento, tenemos el siguiente hamiltoniano, $\mathcal{H}: \mathbf{E} \longrightarrow \mathbb{R}$, dado por

$$
\mathcal{H}=\frac{1}{2}\left(p_{r}^{2}+\frac{p_{\theta}^{2}}{r^{2}}\right)+\alpha p_{\theta}-\frac{1}{r}+\frac{\beta}{r^{2}}
$$

donde $-\frac{1}{r}+\frac{\beta}{r^{2}}$ es el potencial de Manev y el término $\alpha p_{\theta}$ añade a la dinámica del problema de Kepler el efecto asociado a la rotación del sistema de referencia. Los parámetros $\alpha>0$ y $\beta \in \mathbb{R}$ son dos constantes estructurales del sistema y $\mathbf{E}=\mathbb{R}^{+} \times S^{1} \times \mathbb{R}^{2}$ es el espacio fásico. 
Con idea de hacer un estudio cualitativo de la dinámica asociada a el sistema hamiltoniano, de un modo parecido al que se hace en Llibre (2001) [36], consideramos los siguientes conjuntos:

$$
\begin{gathered}
E_{h}=\mathcal{H}^{-1}(h)=\left\{\left(r, \theta, p_{r}, p_{\theta}\right) \in \mathbf{E}: g\left(r, p_{r}, p_{\theta}\right)=h\right\} \approx g^{-1}(h) \times S^{1}, \\
J_{k}=\left\{z \in \mathbf{E}: p_{\theta}=k\right\}, \\
I_{h k}=E_{h} \cap J_{k} \approx\left(g^{-1}(h) \cap\left\{p_{\theta}=k\right\}\right) \times S^{1},
\end{gathered}
$$

donde $g: \mathbb{R}^{+} \times \mathbb{R}^{2} \longrightarrow \mathbb{R}$ está definida por $g\left(r, p_{r}, p_{\theta}\right)=\mathcal{H}\left(r, \theta, p_{r}, p_{\theta}\right)$ y $g^{-1}(h)$ es una superficie de $\mathbb{R}^{+} \times \mathbb{R}^{2}$ denminada superficie de energía.

Estos conjuntos son invariantes para el flujo asociado a (5.1), siendo $\mathcal{H}$ y $p_{\theta}$ dos integrales primeras del movimiento, independientes y en involución.

Los principales resultados que se obtienen en este capítulo son

a) La descripción de la folación del espacio fásico $\mathbf{E}$ por medio de los conjuntos de energía constante $E_{h}$.

b) La de los conjuntos $E_{h}$ por medio de los conjuntos invariantes $I_{h k}$.

c) Y la foliación de $I_{h k}$ por medio del flujo del sistema hamiltoniano.

Estas foliaciones nos darán una buena descripción del espacio de fases cuando $(h, k) \in$ $\mathbb{R}^{2}$ y cuando depende de los diferentes valores de $\alpha$ y $\beta$.

La herramienta principal de este estudio es el teorema de Liouville-Arnold (véase [6] para más detalles), éste nos permite estudiar la apliación momento, $\left(\mathcal{H}, p_{\theta}\right): \mathbf{E} \times \mathbb{R} \longrightarrow \mathbb{R}^{2}$, en los valores regulares de ella.

Cuando no estemos en condiciones de usar el teorema de Liouville-Arnold theorem se hará un estudio particular de los conjuntos $I_{h k}$ para cada uno de los valores críticos $(h, k) \in \mathbb{R}^{2}$ de la apliación momento. Estos valores corresponderán, bien a los puntos de equilibrio de $\mathcal{H}$, bien a los valores donde $p_{\theta}=k$ es un máximo o un mínimo de la superficie de energía.

\subsection{Potencial ampliado. Regiones de Hill}

Las regiones de Hill están completamente caracterizadas por medio del potencial ampliado (véase Abraham y Marsden (1978) [2] para más detalles). El hamiltoniano (5.1), en coordenadas polares-simplécticas puede escribirse como sigue

$$
\mathcal{H}=\frac{1}{2}\left(p_{r}^{2}+\frac{1}{r^{2}}\left(p_{\theta}+\alpha r^{2}\right)^{2}\right)+\tilde{\mathcal{V}}
$$

de esta manera, al completar cuadrados, obtenemos que el potencial ampliado tiene la siguiente expresión

$$
\tilde{\mathcal{V}}=-\frac{\alpha^{2} r^{2}}{2}-\frac{1}{r}+\frac{\beta}{r^{2}}
$$

Las regiones del espacio fásico donde existe movimiento real están determinadas por medio de los puntos críticos de $\widetilde{\mathcal{V}}$, que corresponden con las raíces reales positivas de la ecuación polinómica

$$
\alpha^{2} r^{4}+r-2 \beta=0
$$


Usando el algoritmo de Sturm obtenemos la siguiente clasificación para la existencia de valores críticos (v. c.), estos dependen de los diferentes valores de $2048 \alpha^{2} \beta^{3}-27$ y $\beta$.

\begin{tabular}{|l|l|l|}
\hline $\mathbf{1}^{e r}$ caso & $\beta>0$ y $2048 \alpha^{2} \beta^{3}-27>0$ & 0 v. c. \\
\hline $\mathbf{2}^{o}$ caso & $\beta>0$ y $2048 \alpha^{2} \beta^{3}-27=0$ & 1 v. c. \\
\hline $\mathbf{3}^{e r}$ caso & $\beta>0$ y $2048 \alpha^{2} \beta^{3}-27<0$ & 2 v. c. \\
\hline $\mathbf{4}^{o}$ caso & $\beta \leq 0$ & 1 v. c.. \\
\hline
\end{tabular}

Tabla 5.1: Clasificación de valores críticos (v. c.) según los diferentes valores de $2048 \alpha^{2} \beta^{3}-27$ y $\beta$.

Para cada $h \in \mathbb{R}$, definimos las regiones de Hill $R_{h}$ de $E_{h}$ por $R_{h}=\pi\left(E_{h}\right)$, donde $\pi: \mathbf{E} \longrightarrow \mathbb{R}^{+} \times S^{1}$ es la proyección natural. Así pues,

$$
R_{h}=\left\{(r, \theta) \in \mathbb{R}^{+} \times S^{1}: \widetilde{\mathcal{V}} \leq h\right\} \approx\left\{r \in \mathbb{R}^{+}:-\frac{\alpha^{2} r^{2}}{2}-\frac{1}{r}+\frac{\beta}{r^{2}} \leq h\right\} \times S^{1},
$$

donde $\approx$ significa difeomorfo a.

\subsection{Topología de las regiones de Hill}

De ahora en adelante los valores del potencial ampliado, en cada uno de sus puntos críticos $r_{i}$, serán denotados por $h_{i}=\widetilde{\mathcal{V}}\left(r_{i}\right),(i=1,2,3)$. Los valores $A_{j},(j=1, \ldots, 7)$, corresponderán a los puntos de intersección de la gráfica del potencial y la recta $\widetilde{\mathcal{V}}=h$.

Con idea de hacer más claro el estudio de la topología de las regiones de Hill se presentan las siguientes figuras

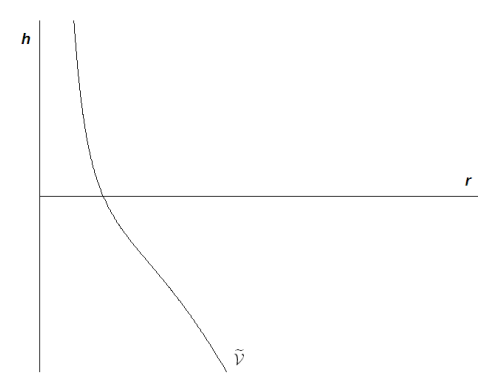

Figura 5.1: Potencial ampliado para $\beta>$ $0 \mathrm{y}-27 / 2048+\alpha^{2} \beta^{3}>0$.

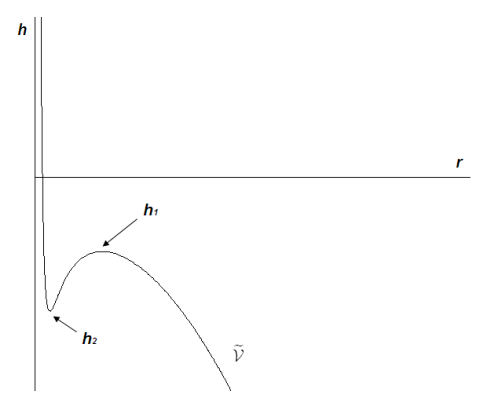

Figura 5.3: Potencial ampliado para $\beta>$ $0 \mathrm{y}-27 / 2048+\alpha^{2} \beta^{3}<0$.

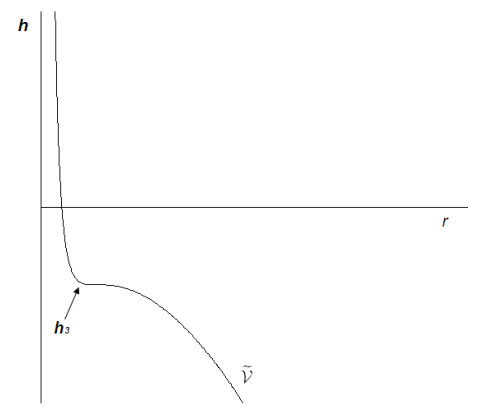

Figura 5.2: Potencial ampliado para $-27 / 2048+\alpha^{2} \beta^{3}=0$.

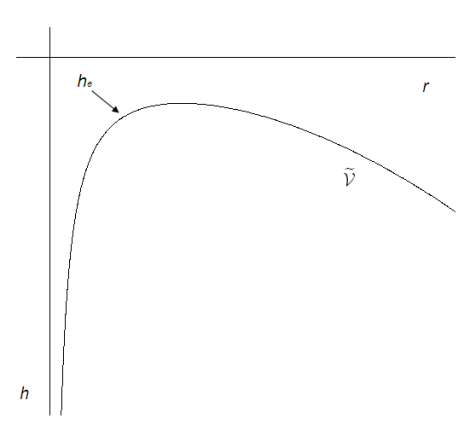

Figura 5.4: Potencial ampliado para $\beta=0$. 


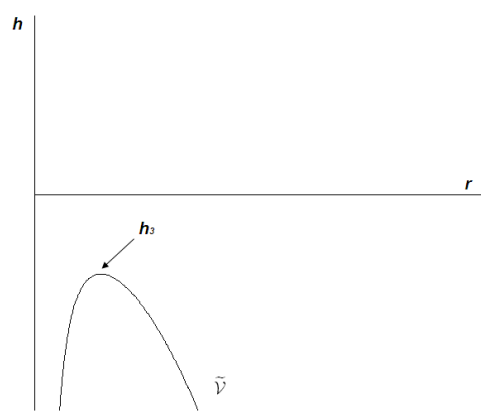

Figura 5.5: Potencial ampliado para $\beta<0$.

Usando los resultados que se han obtenido previamente, la clasificación topológica de las regiones de Hill queda como sigue

Primer caso: $\beta>0$ and $-27+2048 \alpha^{2} \beta^{3}>0$.

\begin{tabular}{|l|l|l|}
\hline$\beta>0,-27+2048 \alpha^{2} \beta^{3}>0$ & $\forall h$ & $R_{h} \approx\left[A_{1},+\infty\right] \times S^{1}$ \\
\hline
\end{tabular}

Tabla 5.2: Clasificación topológica de las regiones de Hill cuando $\beta>0$ y $-27+2048 \alpha^{2} \beta^{3}>0$ (véase 5.1).

Segundo caso: $-27+2048 \alpha^{2} \beta^{3}=0$.

\begin{tabular}{|l|l|l|}
\hline \multirow{2}{*}{$-27+2048 \alpha^{2} \beta^{3}=0$} & $h_{1}<h$ & $R_{h} \approx\left[A_{1},+\infty\right] \times S^{1}$ \\
\cline { 2 - 3 } & $h=h_{1}$ & $R_{h} \approx\left[h_{1},+\infty\right) \times S^{1}$ \\
\cline { 2 - 3 } & $h<h_{1}$ & $R_{h} \approx\left[A_{2},+\infty\right) \times S^{1}$ \\
\hline
\end{tabular}

Tabla 5.3: Clasificación topológica de las regiones de Hill cuando $-27 / 2048+\alpha^{2} \beta^{3}=0$ (véase $5.2)$. 
Tercer caso: $\beta>0$ and $-27+2048 \alpha^{2} \beta^{3}<0$.

\begin{tabular}{|l|l|l|}
\hline \multirow{3}{*}{$\beta>0,-27+2048 \alpha^{2} \beta^{3}<0$} & $h_{2}<h$ & $R_{h} \approx\left[A_{1},+\infty\right) \times S^{1}$ \\
\cline { 2 - 3 } & $h=h_{2}$ & $R_{h} \approx\left[h_{2},+\infty\right) \times S^{1}$ \\
\cline { 2 - 3 } & $h_{3}<h<h_{2}$ & $R_{h} \approx\left\{\left[A_{2}, A_{3}\right] \cup\left[A_{4},+\infty\right)\right\} \times S^{1}$ \\
\cline { 2 - 3 } & $h=h_{3}$ & $R_{h} \approx\left[\left\{h_{3}\right\} \cup\left[A_{5},+\infty\right)\right] \times S^{1}$ \\
\cline { 2 - 3 } & $h \leq h_{3}$ & $R_{h} \approx\left[A_{6},+\infty\right) \times S^{1}$ \\
\hline
\end{tabular}

Tabla 5.4: Clasificación topológica de las regiones de Hill cuando $\beta>0$ y $-27+2048 \alpha^{2} \beta^{3}<0$ (véase 5.3).

Cuarto caso: $\beta=0$.

\begin{tabular}{|l|l|l|}
\hline \multirow{2}{*}{$b=0$} & $h_{4} \leq h$ & $R_{h} \approx(0,+\infty) \times S^{1}$ \\
\cline { 2 - 3 } & $h<h_{4}$ & $R_{h} \approx\left[\left(0, A_{1}\right] \cup\left[A_{2},+\infty\right)\right] \times S^{1}$ \\
\hline
\end{tabular}

Tabla 5.5: Clasificación topológica de las regiones de Hill cuando $\beta=0$ (véase 5.4).

Quinto caso: $\beta<0$.

\begin{tabular}{|l|l|l|}
\hline \multirow{2}{*}{$b<0$} & $h_{5} \leq h$ & $R_{h} \approx(0,+\infty) \times S^{1}$ \\
\cline { 2 - 3 } & $h<h_{5}$ & $R_{h} \approx\left[\left(0, A_{1}\right] \cup\left[A_{2},+\infty\right)\right] \times S^{1}$ \\
\hline
\end{tabular}

Tabla 5.6: Clasificación topológica de las regiones de Hill cuando $\beta<0$ (véase 5.5). 


\subsection{Estudio cualitativo del flujo hamiltoniano}

En esta sección estudiamos la topología de las vaiedades invariantes $\mathcal{H}^{-1}(h)=E_{h}$ e $I_{h k}$. Para poder dar esta clasificación necesitamos introducir la siguiente notación e incluir algunos resultados nuevos.

En primer lugar, nótese que $z_{e}=\left(r_{e}, \theta_{e}, p_{r_{e}}, p_{\theta_{e}}\right) \in \mathbf{E}$ es un punto de equilibrio del fujo hamiltoniano si y sólo si $\tilde{z}_{e}=\left(r_{e}, \theta_{e}\right)$ es un punto crítico del potencial ampliado. Más aún, $\pi\left(z_{e}\right)=\tilde{z}_{e}$. Es por esta razón que cuando $\beta>0$ y $2048 \alpha^{2} \beta^{3}-27>0$ el hamiltoniano no tiene equilibrios; si $\beta>0$ y $2048 \alpha^{2} \beta^{3}-27=0$ el hamiltoniano tiene una familia de puntos de equilibrios; si $\beta>0$ y $2048 \alpha^{2} \beta^{3}-27<0$ el hamiltoniano tiene dos. Finalmente, cuando $\beta \leq 0$ el hamiltoniano tiene una única familia de puntos de equilibrios.

Así pues, podemos denotar de la misma manera que denotábamos el valor del potencial ampliado en cada uno de sus puntos críticos, por $h_{i}=\mathcal{H}\left(r_{i}, \theta_{i}, 0, p_{\theta_{i}}\right)(i=1,2,3,4)$, a los valores del hamiltoniano $\mathcal{H}$ en cada uno de sus puntos de equilibrio.

Recordemos que llamamos a $g^{-1}(h)$ superficie de energía. Usando el teorema de la función implícita se puede ver que los extremos de la superficie de energía son las raíces reales de la siguiente ecuación

$$
2 \alpha k^{3}-2 h k^{2}+4 \alpha \beta k-4 \beta h-1=0 .
$$

Estas raíces las llamamos $c_{j}$ y corresponden a los extremos de la superficie de energía que denotamos por $Q_{j},(j=1,2,3)$.

Denotamos por $a_{i}$, a las raíces reales respecto de $k$ de las ecuaciones $h-\alpha k=0 \mathrm{y}$ $2 \beta+k^{2}=0$, las cuales se obtienen aplicando el algoritmo de Sturm a (5.3). Estos valores nos dicen dónde las trayectorias no son acotadas. En el resto de los casos o no tenemos superficie de energía, o es un punto o un trayectoria acotada. Finalmente, sea $S^{n-1}$ la esfera de $\mathbb{R}^{n}$, con $n>1$

Los diferentes casos pueden verse por medio de las siguientes tablas y sus correspondientes figuras. 
$\underline{\text { Primer caso }} \beta>0 \mathrm{y}-27+2048 \alpha^{2} \beta^{3}>0$.

\begin{tabular}{|c|c|ll|}
\hline \multicolumn{1}{|c|}{$h$} & \multicolumn{1}{|c|}{$E_{h}$} & \multicolumn{2}{|c|}{$I_{h k}$} \\
\hline \multirow{4}{*}{$\forall h$} & & $\emptyset$ & $c_{1}<k$ \\
& $S^{2} \backslash\left\{S^{1} \cup S^{1}\right\}($ véase 5.6) & $S^{1}$ & $k=c_{1}$ \\
& & $S^{1} \times S^{1}$ & $a_{1}<k<c_{1}$ \\
& & $S^{1} \times \mathbb{R} \quad k \leq a_{1}$ \\
\hline
\end{tabular}

Tabla 5.7: Clasificación topológica de $E_{h}$ e $I_{h k}$ cuando $\beta>0$ y $-27+2048 \alpha^{2} \beta^{3}>0$.

Segundo caso: $-27+2048 \alpha^{2} \beta^{3}=0$.

\begin{tabular}{|c|c|ll|}
\hline \multicolumn{1}{|c|}{$E_{h}$} & \multicolumn{2}{|c|}{$I_{h k}$} \\
\hline \multirow{5}{*}{$h_{1}<h$} & \multirow{2}{*}{$S^{2} \backslash\left\{S^{1} \cup S^{1}\right\}$ (véase 5.7) } & $\emptyset$ & $c_{1}<k$ \\
& & $S^{1} \times S^{1}$ & $a_{1}<k<c_{1}$ \\
& & $S^{1} \times \mathbb{R}$ & $k \leq a_{1}$ \\
\hline \multirow{5}{*}{$h=h_{1}$} & \multirow{3}{*}{$S^{2} \backslash\left\{S^{1} \cup S^{1}\right\}$ (véase 5.8) } & $\emptyset$ & $h_{1}<k$ \\
& & $S^{1} \times S^{1}$ & $a_{2}<k<h_{1}$ \\
& & $S^{1} \times \mathbb{R}$ & $k \leq a_{2}$ \\
\hline \multirow{5}{*}{$h<h_{1}$} & \multirow{2}{*}{$S^{2} \backslash\left\{S^{1} \cup S^{1}\right\}$ (véase 5.9) } & $\emptyset$ & $c_{1}<k$ \\
& & $S^{1}$ & $k=c_{1}$ \\
& & $S^{1} \times S^{1}$ & $a_{3}<k<c_{1}$ \\
& & $S^{1} \times \mathbb{R}$ & $k \leq a_{3}$ \\
\hline
\end{tabular}

Tabla 5.8: Clasificación topológica de $E_{h}$ e $I_{h k}$ cuando $-27+2048 \alpha^{2} \beta^{3}=0$. 
Tercer caso: $\beta>0 \mathrm{y}-27+2048 \alpha^{2} \beta^{3}<0$.

\begin{tabular}{|c|c|c|c|}
\hline$h$ & $E_{h}$ & $I_{h k}$ & \\
\hline$h_{2}<h$ & $S^{2} \backslash\left\{S^{1} \cup S^{1}\right\}$ (véase 5.10 ) & $\begin{array}{l}\emptyset \\
S^{1} \\
S^{1} \times S^{1} \\
S^{1} \times \mathbb{R}\end{array}$ & $\begin{array}{l}c_{1}<k \\
k=c_{1} \\
a_{1}<k<c_{1} \\
k \leq a_{1}\end{array}$ \\
\hline$h=h_{2}$ & $S^{2} \cup S^{0} \cup\left\{S^{2} \backslash\left\{S^{1} \cup S^{1}\right\}\right\}($ véase 5.11) & $\begin{array}{l}\emptyset \\
S^{1} \\
S^{1} \times S^{1} \\
S^{1} \\
S^{1} \times S^{1} \\
S^{1} \times \mathbb{R}\end{array}$ & $\begin{array}{l}c_{1}<k \\
k=c_{1} \\
h_{2}<k<c_{1} \\
k=h_{2} \\
a_{2}<k<h_{2} \\
k \leq a_{2}\end{array}$ \\
\hline$h_{3}<h<h_{2}$ & $S^{2} \cup\left\{S^{2} \backslash\left\{S^{1} \cup S^{1}\right\}\right\}$ (véase 5.12) & $\begin{array}{l}\emptyset \\
S^{1} \\
S^{1} \times S^{1} \\
S^{1} \\
\emptyset \\
S^{1} \\
S^{1} \times S^{1} \\
S^{1} \times \mathbb{R}\end{array}$ & $\begin{array}{l}c_{1}<k \\
k=c_{1} \\
c_{2}<k<c_{1} \\
k=c_{2} \\
c_{3}<k<c_{2} \\
k=c_{3} \\
a_{3}<k<c_{3} \\
k \leq a_{3}\end{array}$ \\
\hline$h=h_{3}$ & $S^{0} \cup\left\{S^{2} \backslash\left\{S^{1} \cup S^{1}\right\}\right\}$ (véase 5.13 ) & $\begin{array}{l}\emptyset \\
S^{1} \\
\emptyset \\
S^{1} \\
S^{1} \times S^{1} \\
S^{1} \times \mathbb{R}\end{array}$ & $\begin{array}{l}h_{3}<k \\
k=h_{3} \\
c_{1}<k<h_{3} \\
k=c_{1} \\
a_{4}<k<c_{1} \\
k \leq a_{4}\end{array}$ \\
\hline$h \leq h_{3}$ & $S^{2} \backslash\left\{S^{1} \cup S^{1}\right\}$ (véase 5.14 ) & $\begin{array}{l}\emptyset \\
S^{1} \\
S^{1} \times S^{1} \\
S^{1} \times \mathbb{R}\end{array}$ & $\begin{array}{l}c_{1}<k \\
k=c_{1} \\
a_{5}<k<c_{1} \\
k \leq a_{5}\end{array}$ \\
\hline
\end{tabular}

Tabla 5.9: Clasificación topológica de $E_{h}$ e $I_{h k}$ cuando $\beta>0$ y $-27+2048 \alpha^{2} \beta^{3}<0$. 
Cuarto caso: $\beta=0$.

\begin{tabular}{|c|c|c|c|}
\hline$h$ & $E_{h}$ & \multicolumn{2}{|l|}{$I_{h k}$} \\
\hline$h_{4}<h$ & $\left\{S^{2} \backslash\left\{S^{1} \cup S^{1} \cup S^{1}\right\}\right\}(5.15)$ & $\begin{array}{l}\emptyset \\
S^{1} \\
S^{1} \times S^{1}, \\
S^{1} \times \mathbb{R}, \\
S^{1} \times S^{1}, \\
S^{1} \times \mathbb{R},\end{array}$ & $\begin{array}{l}c_{1}<k \\
k=c_{1} \\
0<k<c_{1} \\
k=0 \\
a_{1}<k<0 \\
k \leq a_{1}\end{array}$ \\
\hline$h=h_{4}$ & $\left\{S^{2} \backslash S^{1}\right\} \cup\left\{S^{0}\right\} \cup\left\{S^{2} \backslash\left\{S^{1} \cup S^{1}\right\}\right\}$ & $\begin{array}{l}\emptyset \\
S^{1} \\
S^{1} \times S^{1}, \\
S^{1} \times \mathbb{R}, \\
S^{1} \times S^{1}, \\
S^{1} \\
S^{1} \times S^{1} \\
S^{1} \times \mathbb{R},\end{array}$ & $\begin{array}{l}c_{1}<k \\
k=c_{1} \\
0<k<c_{1} \\
k=0 \\
h_{4}<k<0 \\
k=h_{4} \\
a_{2}<k<h_{4} \\
k \leq a_{2}\end{array}$ \\
\hline$h<h_{4}$ & $\left\{S^{2} \backslash S^{1}\right\} \cup\left\{S^{2} \backslash\left\{S^{1} \cup S^{1} \cup S^{1}\right\}\right\}$ & $\begin{array}{l}\emptyset \\
S^{1} \\
S^{1} \times S^{1}, \\
S^{1} \times \mathbb{R}, \\
S^{1} \times S^{1}, \\
\emptyset \\
S^{1} \\
S^{1} \times S^{1}, \\
S^{1} \times \mathbb{R},\end{array}$ & $\begin{array}{l}c_{1}<k \\
k=c_{1} \\
0<k<c_{1} \\
k=0 \\
c_{2}<k<0 \\
c_{3}<k<c_{2} \\
k=c_{3} \\
c_{3}<k<a_{3} \\
k \leq a_{3}\end{array}$ \\
\hline
\end{tabular}

Tabla 5.10: Clasificación topológica de $E_{h}$ e $I_{h k}$ cuando $\beta=0$. 
Quinto caso: $\beta<0$.

\begin{tabular}{|c|c|c|c|}
\hline$h$ & $E_{h}$ & \multicolumn{2}{|l|}{$I_{h k}$} \\
\hline$h_{5}<h$ & $S^{2} \backslash\left\{S^{1} \cup S^{1}\right\}$ (véase 5.18 ) & $\begin{array}{l}\emptyset \\
S^{1} \\
S^{1} \times S^{1} \\
S^{1} \times \mathbb{R}\end{array}$ & $\begin{array}{l}c_{1}<k \\
k=c_{1} \\
\sqrt{2}<k<c_{1} \\
k \leq \sqrt{2}\end{array}$ \\
\hline$h=h_{5}$ & $\left\{S^{2} \backslash\left\{S^{1} \cup S^{1} \cup S^{1}\right\}\right\} \cup S^{0} \cup\left\{S^{2} \backslash\left\{S^{1} \cup S^{1}\right\}\right\}$ (véase 5.19) & $\begin{array}{l}\emptyset \\
S^{1} \\
S^{1} \times S^{1} \\
S^{1} \times \mathbb{R} \\
S^{1} \times S^{1} \\
S^{1} \times \mathbb{R}\end{array}$ & $\begin{array}{l}c_{1}<k \\
k=c_{1} \\
\sqrt{2}<k<c_{1} \\
-\sqrt{2}<k \leq \sqrt{2} \\
a_{1}<k \leq-\sqrt{2} \\
k \leq a_{1}\end{array}$ \\
\hline$h<h_{5}$ & $\left\{S^{2} \backslash\left\{S^{1} \cup S^{1} \cup S^{1}\right\}\right\} \cup\left\{S^{2} \backslash\left\{S^{1} \cup S^{1}\right\}\right\}$ (véase 5.20) & $\begin{array}{l}\emptyset \\
S^{1} \\
S^{1} \times S^{1} \\
S^{1} \times \mathbb{R} \\
S^{1} \times S^{1} \\
\emptyset \\
S^{1} \\
S^{1} \times S^{1} \\
S^{1} \times \mathbb{R}\end{array}$ & $\begin{array}{l}c_{1}<k \\
k=c_{1} \\
\sqrt{2}<k<c_{1} \\
-\sqrt{2}<k \leq \sqrt{2} \\
c_{2}<k \leq-\sqrt{2} \\
c_{3}<k<c_{2} \\
k=c_{3} \\
a_{2}<k<c_{3} \\
k \leq a_{2}\end{array}$ \\
\hline
\end{tabular}

Tabla 5.11: Clasificación topológica de $E_{h}$ e $I_{h k}$ cuando $\beta<0$. 


\section{Primer caso:}

Figuras que corresponden a la superficie de energía para $\beta>0$ y $-27+2048 \alpha^{2} \beta^{3}>0$.

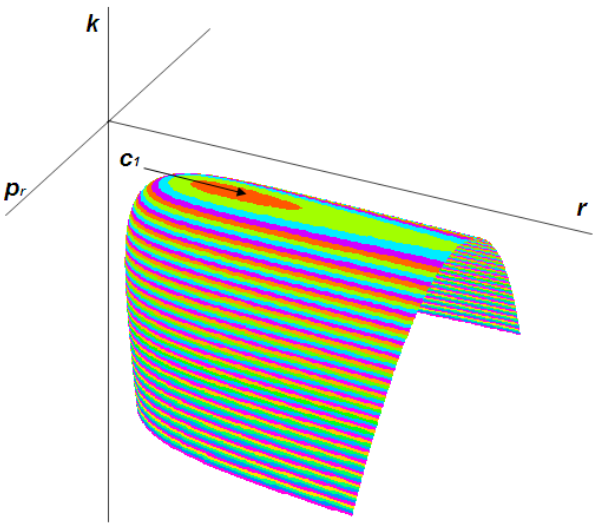

Figura 5.6: $\mathcal{H}^{-1}(h) / S^{1}$ para $\beta>0,-27 / 2048+\alpha^{2} \beta^{3}$ y $\forall h$, donde $k=p_{\theta}$, y $c_{1}$ el máximo.

\section{Segundo caso:}

Figuras que corresponden a la superficie de energía para $-27+2048 \alpha^{2} \beta^{3}=0$.

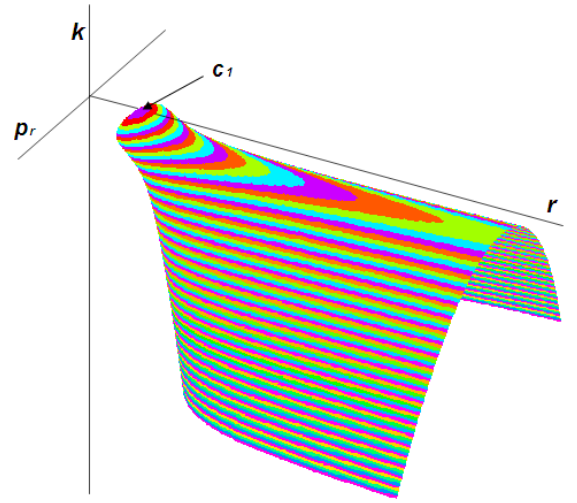

Figura 5.7: $\mathcal{H}^{-1}(h) / S^{1}$ para $-27 / 2048+$ $\alpha^{2} \beta^{3}=0$ y $h_{1}<h$, donde $k=p_{\theta}$, y $c_{1}$ el máximo.

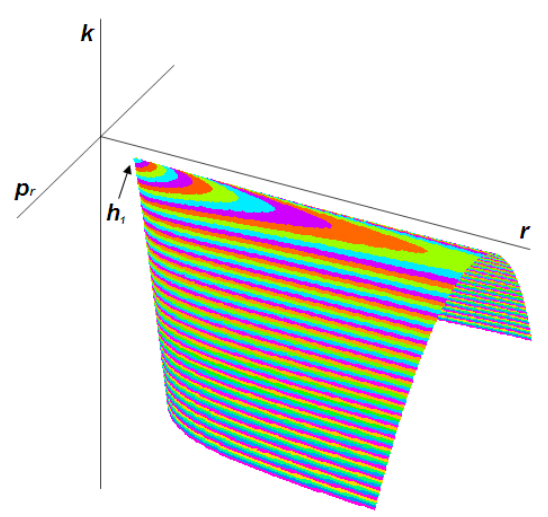

Figura 5.8: $\mathcal{H}^{-1}(h) / S^{1}$ para $-27 / 2048+$ $\alpha^{2} \beta^{3}=0$ y $h=h_{1}$, donde $k=p_{\theta}$ y $h_{1}$ es el punto de equilibrio. 


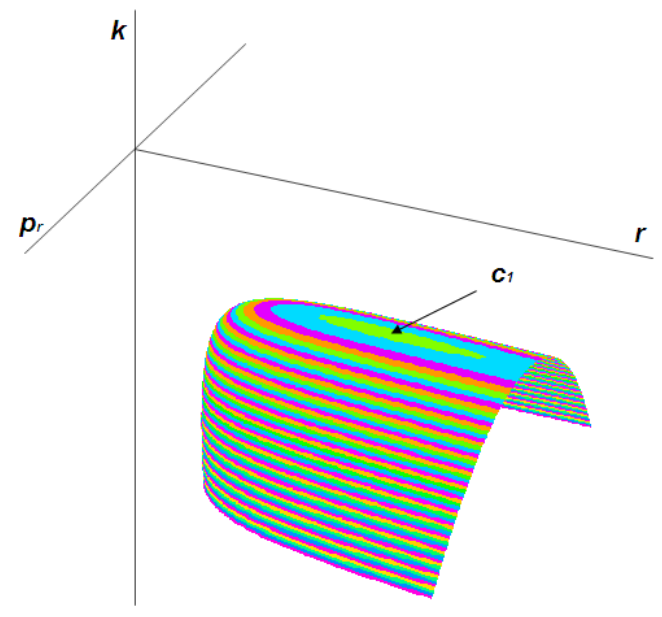

Figura 5.9: $\mathcal{H}^{-1}(h) / S^{1}$ para $-27 / 2048+\alpha^{2} \beta^{3}=0$ y $h<h_{1}$, donde $k=p_{\theta}$, y $c_{1}$ el máximo.

\section{Tercer caso:}

Figuras que corresponden a la superficie de energía para $\beta>0$ y $-27+2048 \alpha^{2} \beta^{3}<0$.

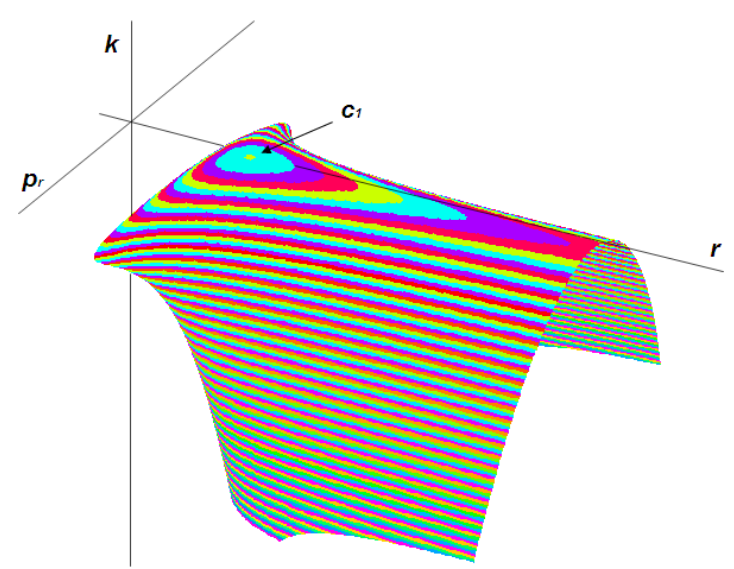

Figura 5.10: $\mathcal{H}^{-1}(h) / S^{1}$ para $\beta>0$, $-27 / 2048+\alpha^{2} \beta^{3}<0$ y $h_{2}<h$, donde $k=p_{\theta}$ y $c_{1}$ el máximo.

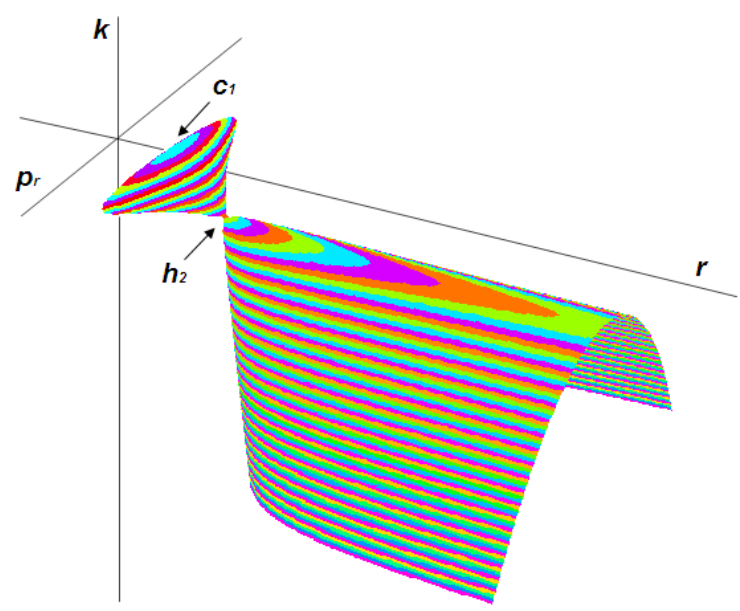

Figura 5.11: $\mathcal{H}^{-1}(h) / S^{1}$ para $\beta>0$, $-27 / 2048+\alpha^{2} \beta^{3}<0$ y $h=h_{2}$, donde $k=p_{\theta}$ y $c_{1}$ el máximo. 


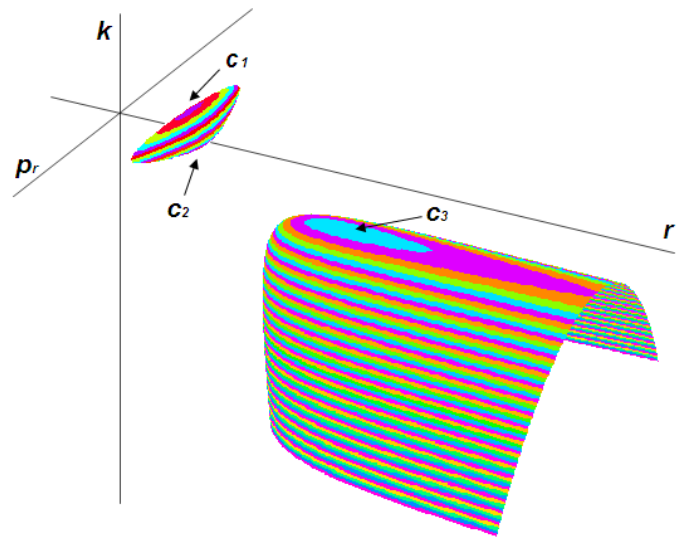

Figura 5.12: $\mathcal{H}^{-1}(h) / S^{1}$ para $\beta>0$, $-27 / 2048+\alpha^{2} \beta^{3}<0$ y $h_{3}<h<h_{2}$, donde $k=p_{\theta}$, y $c_{1}, c_{2}, c_{3}$ los extremos.

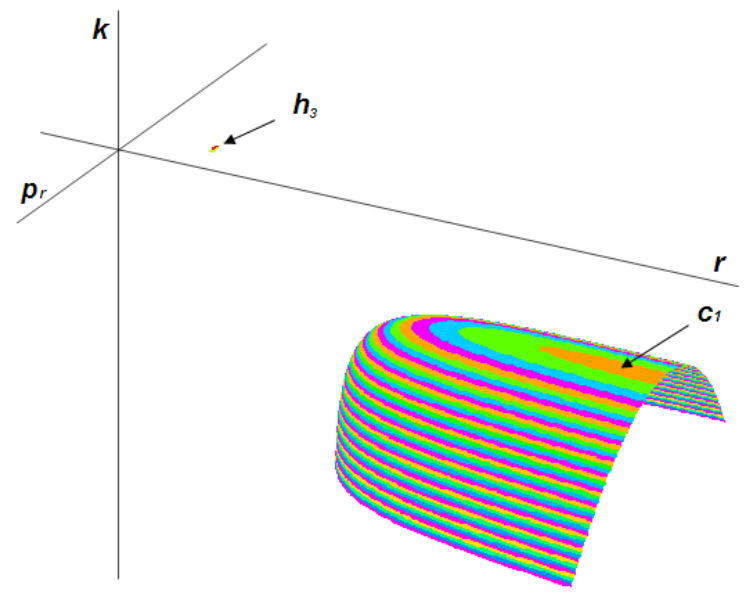

Figura 5.13: $\mathcal{H}^{-1}(h) / S^{1}$ para $\beta>0,27 / 2048+$ $\alpha^{2} \beta^{3}<0$ y $h=h_{3}$, donde $k=p_{\theta}, h_{3}$ el punto de equilibrio y $c_{1}$ el máximo.

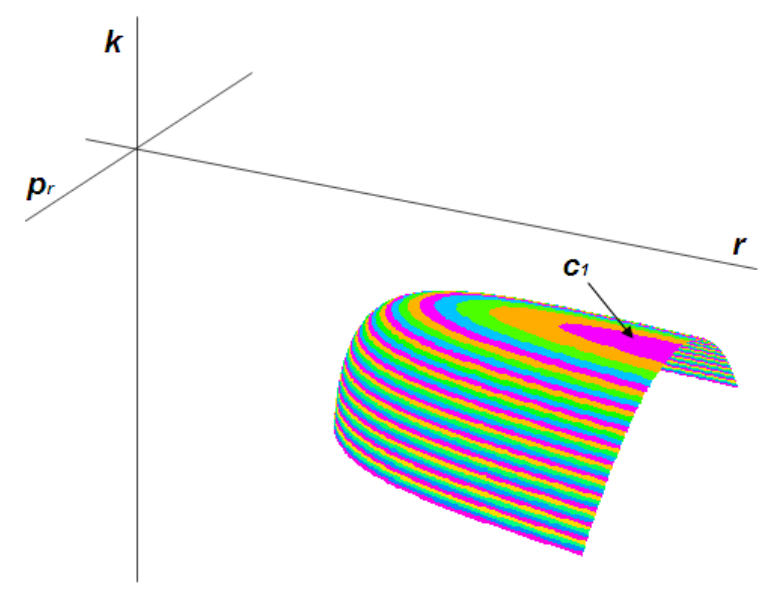

Figura 5.14: $\mathcal{H}^{-1}(h) / S^{1}$ para $\beta>0,-27 / 2048+\alpha^{2} \beta^{3}<0$ y $h \leq h_{3}$, donde $k=p_{\theta}$ y $c_{1}$ el máximo. 


\section{Cuarto caso:}

Figuras que corresponden a la superficie de energía para $\beta=0$.

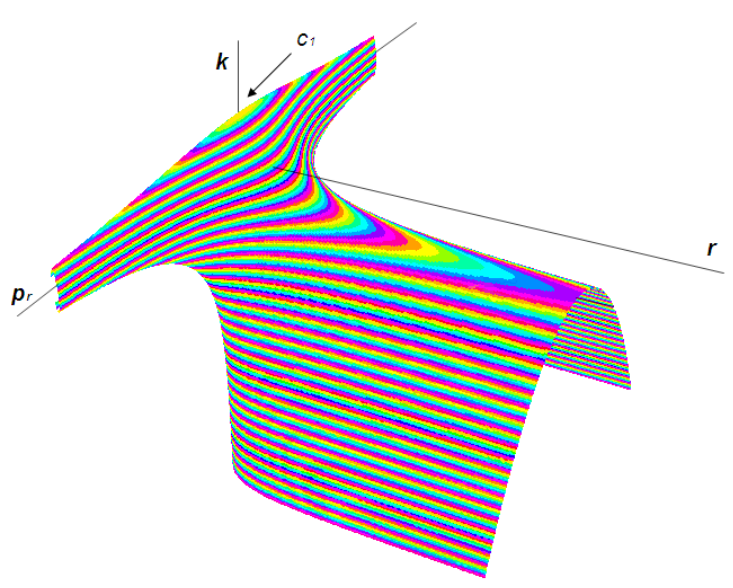

Figura 5.15: $\mathcal{H}^{-1}(h) / S^{1}$ para $\beta=0 \mathrm{y}$ $h_{4}<h$, donde $k=p_{\theta}$ y $c_{1}$ el máximo.

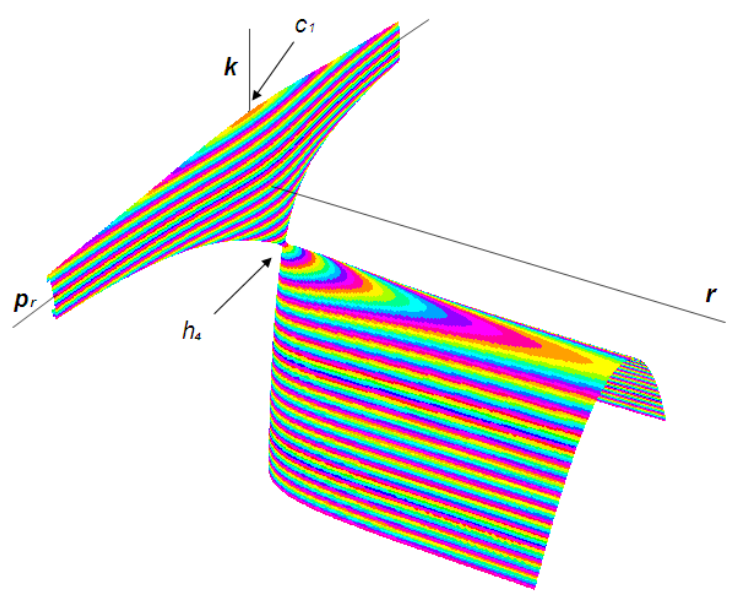

Figura 5.16: $\mathcal{H}^{-1}(h) / S^{1}$ para $\beta=0 \mathrm{y}$ $h=h_{4}$, donde $k=p_{\theta}, h_{4}$ el punto de equilibrio y $c_{1}$ el máximo.

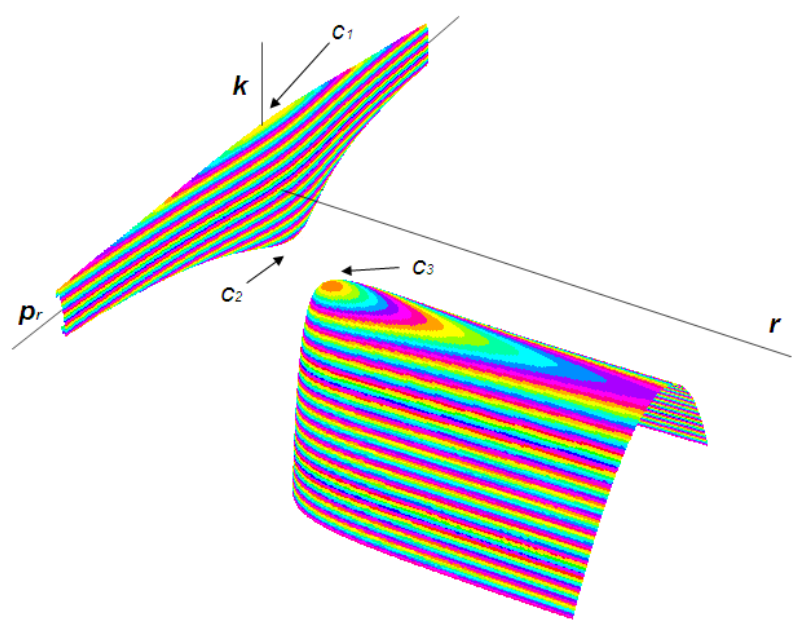

Figura 5.17: $\mathcal{H}^{-1}(h) / S^{1}$ para $\beta=0$ y $h<h_{4}$, donde $k=p_{\theta}$ y $c_{1}, c_{2}, c_{3}$ los extremos. 


\section{Quinto caso:}

Figuras que corresponden a la superficie de energía para $\beta<0$.

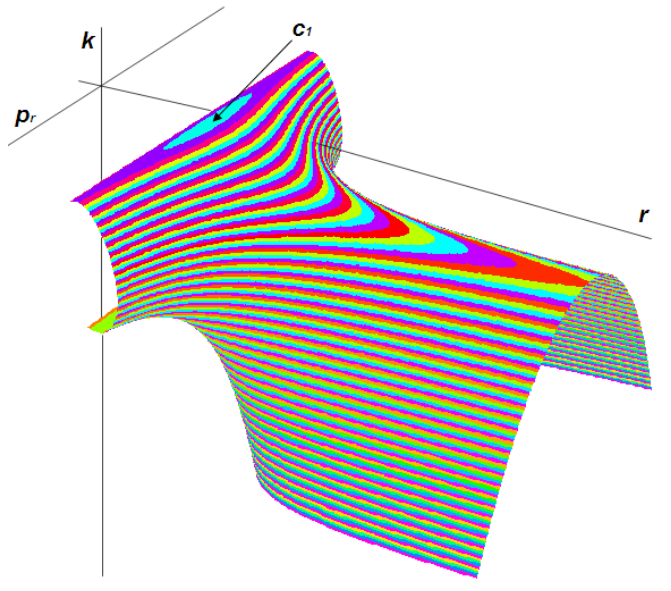

Figura 5.18: $\mathcal{H}^{-1}(h) / S^{1}$ para $\beta<0$ y $h_{5}<h$, donde $k=p_{\theta}$ y $c_{1}$ el máximo.

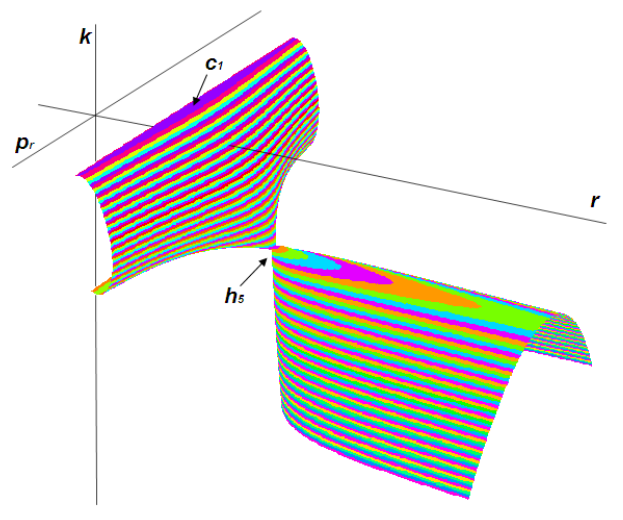

Figura 5.19: $\mathcal{H}^{-1}(h) / S^{1}$ para $\beta<0$ y $h=$ $h_{5}$, donde $k=p_{\theta}, h_{5}$ el punto de equilibrio y $c_{1}$ el máximo.

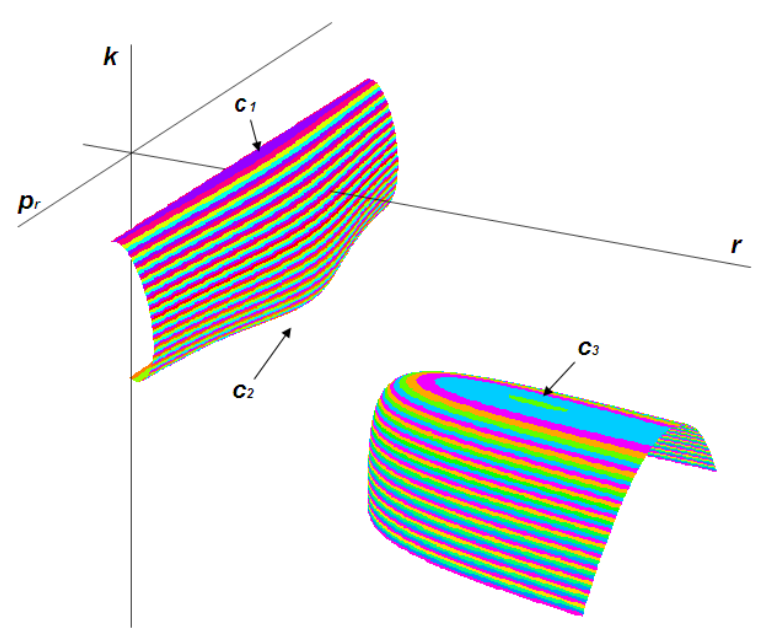

Figura 5.20: $\mathcal{H}^{-1}(h) / S^{1}$ para $\beta<0$ y $h<h_{5}$, donde $k=p_{\theta}$ y $c_{1}, c_{2}, c_{3}$ los extremos. 
Nótese que, en el caso de $\beta=0$, tenemos el problema de Kepler en un sistema de referencia rotante.

\subsection{Variables acción-ángulo}

En esta sección calculamos, por medio de la teoría de Hamilton-Jacobi, las variables acción-ángulo así como la expresión de nuestro hamiltoniano en dichas variables. Estas varibles son útiles para calculas los elementos de Kepler y las ecuaciones planetarias que derivan de dichos elementos.

Las variables acción-ángulo vienen definidas por:

$$
J_{\theta}=\frac{1}{2 \pi} \int p_{\theta} d \theta=k
$$

y

$$
J_{r}=\frac{1}{2 \pi} \int \sqrt{\frac{1}{2}\left(p_{r}^{2}+\frac{1}{r^{2}}\left(p_{\theta}+\alpha r^{2}\right)^{2}\right)-\frac{\alpha^{2} r^{2}}{2}-\frac{1}{r}+\frac{\beta}{r^{2}}} d r
$$

donde

$$
\widetilde{\mathcal{V}}(r)=-\frac{\alpha^{2} r^{2}}{2}-\frac{1}{r}+\frac{\beta}{r^{2}}
$$

es la expresión del potencial ampliado en coordenadas polares-simplécticas.

Para poder calcular $J_{r}$ usaremos el teorema de los residuos de Cauchy.

Las expresiones para $J_{\theta}$ y $J_{r}$ vienen dadas por:

$$
\begin{gathered}
J_{\theta}=k \\
J_{r}=-\sqrt{2 \beta+J_{\theta}^{2}}+\frac{1}{\sqrt{2\left(\alpha J_{\theta}-h\right)}},
\end{gathered}
$$

La transformación de coordenadas polares-simplécticas a variables acción-ángulo puede ser definida en las regiones donde la siguiente ecuación en $r$

$$
\frac{1}{2}\left(p_{r}^{2}+\frac{1}{r^{2}}\left(p_{\theta}+\alpha r^{2}\right)^{2}\right)-\frac{\alpha^{2} r^{2}}{2}-\frac{1}{r}+\frac{\beta}{r^{2}}=0
$$

tiene dos raíces reales (que son distintas), de acuerdo a los parámetros $\alpha, \beta$ y $h$.

Por medio del algoritmo de Sturm obtenemos la región donde (5.6) tiene dos raíces reales distintas. La región viene dada por las siguientes inecuaciones

$$
\begin{gathered}
2 \beta+k^{2}>0, \\
-h+\alpha k>0, \\
2 \beta+k^{2}+\frac{1}{2 h-2 \alpha k}<0 .
\end{gathered}
$$

Dependiendo de $\beta$, distinguimos entre dos regiones 


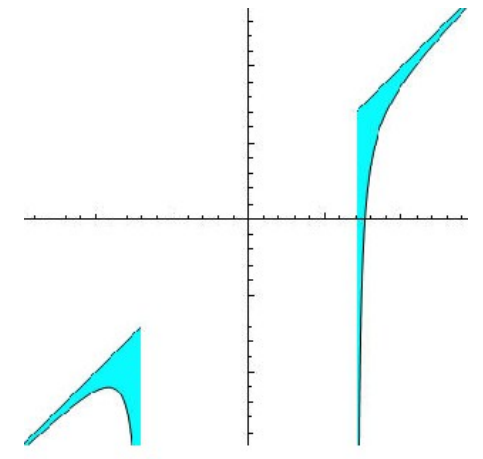

Figura 5.21: Región donde las variables acción-ángle están definidas para $\beta>0$

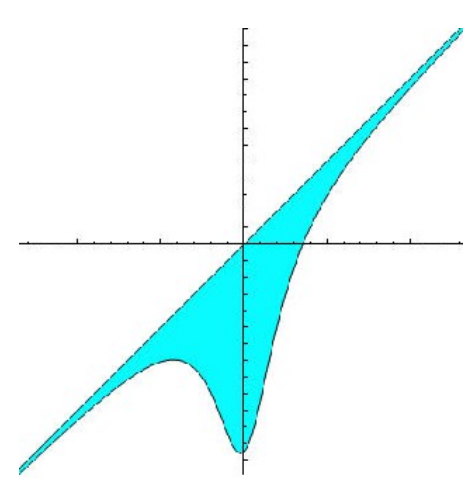

Figura 5.22: Región donde las variables acción-ángle están definidas para $\beta \leq 0$

Resolviendo $h$ en (5.5) y considerando $J_{\theta}=k$ obtenemos el hamiltoniano expresado por medio de estas nuevas variables:

$$
\mathcal{H}=\alpha k+\frac{1}{2\left(2 \beta+J_{r}^{2}+J_{\theta}^{2}+2 J_{r} \sqrt{2 \beta+J_{\theta}^{2}}\right)}
$$

\subsection{Conclusiones}

En este capítulo se ha dado un modo alternativo de estudiar la precesión de Mercurio, a través de la Mecánica Clásica en lugar de la Mecánica Relativista. Fue Manev quien estudió la corrección al potencial atractivo newtoniano, en las últimas décadas numerosos autores también lo han estudiado. En ciertas variedades invariantes, al hamiltoniano asociado al potencial de Manev se le ha sumado un término que lo que hace es añadir a la dinámica del problema kepleriano el efecto asociado a la rotación del sistema de referencia. A través de diferentes técnicas y del teorema de Liouville-Arnold, hemos podido realizar el estudio cualitativo del sistema hamiltoniano en cuestión. Gracias al cálculo de los puntos críiticos del potencial ampliado, hemos podido definir y calcular las regiones de Hill, regiones del espacio fásico donde existe movimiento real, así como su topología. Por último, tras definir lo que hemos llamado superficie de energía, se ha hecho la clasificación topológica de los subconjuntos invariantes $E_{h}$ e $I_{h k}$, y calculado las variables acción-ángulo, junto al hamiltoniano expresado en estas variables, útil para elaborar una teoría de perturbaciones. 



\section{6 \\ Una aproximación al Main Problem del satélite artificial}

\subsection{Introducción}

Los satélites artificiales nacieron durante la Guerra Fría, entre los Estados Unidos y la Unión Soviética, ambos intentaron tanto llegar a la Luna como mandar un satélite en órbita espacial. En mayo del año 1946, el proyecto RAND presentó el informe Preliminary Design of an Experimental World-Circling Spaceship (diseño preliminar de una nave espacial en órbita), en el cual se decía que un vehículo satélite con instrumentación apropiada podía ser una de las herramientas científicas más poderosas del siglo XX.

El 29 de julio de 1955, la Casa Blanca anunció que los Estados Unidos intentarían mandar satélites en la primavera de 1958. Esto llegó a ser un proyecto vanguardista. El 31 de julio, los soviéticos anunciaron que ellos tenían la intención de mandar un satélite en agosto de 1957. La Unión Soviética, desde el puerto espacial de Baikonur, mandó su primer satélite artificial, el primero de la humanidad, el 4 de octubre de 1957; esto supuso un antes y un después en la carrera espacial, haciendo que la Unión Soviética, liderada entonces por Rusia, estuviese por delante de los Estados Unidos. Este programa, llamado Sputnik, al tiempo que se posicionaba satisfactoriamente en órbita, emitió señales radiofónicas en forma de sonidos, demostrado el éxito conseguido por los científicos soviéticos.

Está claro, que, desde el punto de vista que concierne al estudio de la dinámica de satélites artificiales alrededor de un cuerpo celeste, éste tiene muchísima importancia en cuanto a casos de misiones espaciales se refiere. El problema del movimiento de un satélite artificial terrestre es uno de los más estudiados en la Mecánica Celeste.

Actualmente, debido al pequeño tamaño del satélite en comparación con las dimensiones de la órbita, son consideradas nula la influencias del movimiento de rotación en el movimiento orbital, así pues, en el estudio de su rotación alrededor del centro de masas, se asume que es bien conocido el movimiento de este punto. El trabajo más importante publicado en relación a este problema del satélite es realizado en algunas de las dos líneas de estudio previamente mencionadas, incluyendo diferentes casos particulares, desde satélites rígidos y diferentes métodos de integración, o por medio de las variables usadas, la forma hamiltoniana o no de las ecuaciones del movimiento, el método de perturbación usado, el movimiento adoptado por el centro de masas, etc.

En este capítulo, vamos a considerar un caso perturbado del Main Problem del problema de satélite artificial (ASP, artificial satellite problem). Primero veamos de qué versa el Main Problem. Estamos en el caso de un satélie artificial orbitando alrededor de un 
cuepo celeste, en particular un planeta como, por ejemplo. la Tierra. Se va a realizar un estudio analítico, numérico y topológico de la dinámica hamiltoniana para un caso simplificado, donde solo consideraremos el primero y el segundo término dominante del potencial gravitacional.

La función potencial para un cuerpo celeste axialmente simétrico puede escribirse como (véase Cédric (2002) [19])

$$
V(r, \varphi)=-\frac{\mu}{r}+\frac{\mu}{r} \sum_{n=2}^{\infty} \frac{J_{r} R^{n}}{r^{n}} P_{n}(\sin \varphi)
$$

donde $\mu=G M, R$ es una constante estándard, usualmente el radio ecuatorial, $r$ y $\varphi$ son el radio y la latitud de la posición con respecto al centro de masas del cuerpo y $J_{n}$ y $P_{n}$ son los coeficientes de orden $n$ y el polinomio de Legendre, respectivamente.

Asumiremos que nuestro planeta principal es lo que Coffey et al. (1993) [20] llaman Earth-like. Esto es, tomamos $J_{2}>>J_{n}, \forall n>2$. Entonces, lo que vamos a hacer es un truncamiento del potencial a su primer término del sumatorio.

El potencial toma entonces la forma que sigue

$$
V(r, \varphi)=-\frac{\mu}{r}+\frac{\mu}{r} \frac{J_{2} R^{2}}{r^{2}} P_{2}(\sin \varphi)
$$

donde asumiremos que $\varphi=0$, siendo entonces el caso plano (lo que se dice, en el plano ecuatorial) y en consecuencia un potencial integrable.

También tomaremos, después de hacer una normalización, que $\mu=1$ y $R=1$.

Nuestro potencial queda expresado como

$$
V(r, 0)=-\frac{1}{r}+\frac{1}{r} \frac{J_{2}}{r^{2}} P_{2}(\sin 0)
$$

donde sabemos que el polinomio de Legendre de orden 2 es $P_{2}(\sin 0)=$ cte. Denotaremos por $\beta$ al producto de las constantes $J_{2} \cdot P_{2}(\sin \varphi)$. Nuestro potencial expresado en términos de nuestra nueva constante es

$$
V(r, 0)=-\frac{1}{r}+\frac{\beta}{r^{3}}
$$

Es necesario observar que la constante $\beta$ depende del valor de $J_{2}$. Cuando $J_{2}$ es negativa, el planeta es achatado, mientras que, cuando $J_{2}$ es positivo, el planeta es estirado.

El hamiltoniano a considerar es:

$$
H=\frac{1}{2}\left(p_{r}^{2}+\frac{p_{\theta}^{2}}{r^{2}}\right)-\frac{1}{r}+\alpha p_{\theta}+\frac{\beta}{r^{3}}
$$

y corresponde a la dinámica de un satélite artificial orbitando alrededor de un cuerpo celeste, en particular un planeta, para el cual hemos introducido el efecto asociado a la rotación del sistema de referencia. 
En la línea de Vera y Vigueras (2006) [62], la dinámica del movimiento viene dada por el hamiltoniano $\mathcal{H}: \mathbf{E} \longrightarrow \mathbb{R}$, siendo

$$
\mathcal{H}=\frac{1}{2}\left(p_{r}^{2}+\frac{p_{\theta}^{2}}{r^{2}}\right)+\alpha p_{\theta}-\frac{1}{r}+\frac{\beta}{r^{3}}=\mathcal{H}_{\text {Kepler }}+\alpha p_{\theta}+\frac{\beta}{r^{3}}
$$

donde $\mathcal{H}_{\text {Kepler }}$ vemos que representa el hamiltoniano asociado al clásico problema de dos cuerpos y $\alpha p_{\theta}$ es el efecto asociado a la rotación del sistema de referencia $\left(\alpha=\frac{2 \pi}{T}\right.$, con $T=$ período de rotación del satélite alrededor del cuerpo celeste) y $\frac{\beta}{r^{3}}$ es el efecto asociado a la forma del cuerpo celeste. Los parámetros $\alpha>0$ y $\beta \in \mathbb{R}$ son dos constantes estructurales del sistema y $\mathbf{E}=\mathbb{R}^{+} \times S^{1} \times \mathbb{R}^{2}$ es el espacio fásico.

Siguiendo los estudios anteriormente expuestos, para poder estudiar de forma qualitativa la dinámica asociada a nuestro sistema hamiltoniano

$$
\begin{aligned}
\frac{\partial r}{\partial t}=\frac{\partial \mathcal{H}}{\partial p_{r}}, \quad \frac{\partial \theta}{\partial t}= & \frac{\partial \mathcal{H}}{\partial p_{\theta}} \\
\frac{\partial p_{r}}{\partial t}=-\frac{\partial \mathcal{H}}{\partial r}, & \frac{\partial p_{\theta}}{\partial t}=-\frac{\partial \mathcal{H}}{\partial \theta}
\end{aligned}
$$

recordamos los siguientes conjuntos

$$
\begin{gathered}
E_{h}=\left\{\left(r, \theta, p_{r}, p_{\theta}\right) \in \mathbf{E}: \mathcal{H}\left(r, \theta, p_{r}, p_{\theta}\right)=h\right\}, h \in \mathbb{R}, \\
J_{k}=\left\{\left(r, \theta, p_{r}, p_{\theta}\right) \in \mathbf{E}: p_{\theta}=k\right\}, k \in \mathbb{R}, \\
I_{h k}=E_{h} \cap I_{k},
\end{gathered}
$$

con $z=\left(r, \theta, p_{r}, p_{\theta}\right) \in \mathbf{E}$ y $(h, k) \in \mathbb{R}^{2}$.

Estos conjuntos sabemos que son invariantes por el flujo del hamiltoniano asociado a $\mathcal{H}$, al ser $\mathcal{H}$ y $p_{\theta}$ integrales primeras de dicho hamiltoniano en involución, es decir, es un sistema hamiltoniano integrable.

El objetivo de este capítulo es describir la foliación, mediante técnicas topológicas de:

(i) El espacio de fases $\mathbf{E}$ por los conjuntos invariantes $E_{h}$.

(ii) Los conjuntos $E_{h}$ por los conjuntos invariantes $I_{h k}$.

(iii) $I_{h k}$ por el flujo del sistema hamiltoniano.

Esta foliación nos dará una buena descripción del espacio de fases $\mathbf{E}$ cuando $(h, k) \in \mathbb{R}^{2}$ varía para los diferentes valores de los parámetros estructurales $\alpha$ y $\beta$.

Como herramienta principal para este estudio utilizaremos el teorema de LiouvilleArnold, para más detalles acerca de este teorema y de la demostración del mismo véase Arnold (1961) [6], Abraham y Marsden (1978) [2] y Libre (2001) [36]. Consideraremos en lo que sigue la aplicación momento $\left(\mathcal{H}, p_{\theta}\right): \mathbf{E} \times \mathbb{R} \longrightarrow \mathbb{R}^{2}$ asociada a dicho sistema integrable. El teorema de Liouville-Arnold muestra que para sistemas dinámicos hamil- 
tonianos integrables, los conjuntos invariantes asociados a las intersecciones de todas las integrales primeras independientes en involución son generalmente subvariedades del espacio de fases. Más aún, si el flujo de cada una de las subvariedades es completo, entonces esas subvariedades son difeomorfas a la unión de cilindros generalizados y el flujo en ellos es conjugado a un flujo lineal. Además muestra el estudio de la aplicación momento $\left(\mathcal{H}, p_{\theta}\right)$ para valores regulares de esta.

Cuando no estamos bajo las hipótesis del teorema de Liouville-Arnold tenemos que realizar un estudio particular de los conjuntos $I_{h k}$ para valores $(h, k) \in \mathbb{R}^{2}$ críticos de la aplicación momento $\left(\mathcal{H}, p_{\theta}\right)$. Estos se corresponden o bien con puntos de equilibrio de $\mathcal{H}$ o con puntos donde $p_{\theta}=k$ coincide con un máximo o un mínimo de la superficie de energía.

Ahora bien, recordemos que los puntos de equilibrio son los que obtenemos al igualar a cero las ecuaciones del flujo y que los valores críticos de la aplicación momento $\left(\mathcal{H}, p_{\theta}\right)$ son aquellos valores $(h, k)$ donde dicha aplicación no es regular, es decir, no es diferenciable. Cuando $h_{i}$ no sea un valor crítico lo llamaremos valor regular.

Así pues, lo que tratamos de hacer en este capítulo es estudiar:

(i) La topología de los conjuntos invariantes $I_{h k}$ cuando $(h, k) \in \mathbb{R}^{2}$ no es un valor regular de la aplicación $\left(\mathcal{H}, p_{\theta}\right)$, y cómo es el flujo en dichos conjuntos.

(ii) Cómo los conjuntos invariantes $I_{h k}$ seccionan a los niveles de energía $E_{h}$.

(iii) Cómo los niveles de energía $E_{h}$ seccionan el espacio de fases $\mathbf{E}$.

\subsection{Potencial ampliado. Regiones de Hill}

En esta sección vamos a calcular el potencial ampliado y vamos a realizar un estudio de las regiones de Hill, regiones del espacio de configuración donde existe el movimiento real.

Completando cuadrados se puede ver que el potencial ampliado, en coordenadas polares-simplécticas es

$$
\tilde{\mathcal{V}}(r, \theta)=-\frac{\alpha^{2} r^{2}}{2}-\frac{1}{r}+\frac{\beta}{r^{3}}
$$

Las regiones de Hill, están determinadas por los puntos críticos del potencial ampliado $\tilde{\mathcal{V}}$. Diremos que el valor $\tilde{z}_{i}=\left(r_{i}, \theta_{i}\right)$ es un punto crítico de $\tilde{\mathcal{V}}$ si es un extremo para el potencial ampliado. Dichos puntos son las raíces reales de la ecuación polinómica

$$
\alpha^{2} r^{5}-r^{2}+3 \beta=0
$$

obtenida al hacer la parcial de la función potencial $\widetilde{\mathcal{V}}$ respecto de $r$ e igualando a cero.

Discutimos, de acuerdo a los diferentes valores de los parámetros $\alpha$ y $\beta$, el número de raíces reales positivas respecto de $r$ de la ecuación (6.4). Para ello empleamos el método de Sturm. 
Es fácil ver que según el valor de los parámetros $\alpha$ y $\beta$ el potencial ampliado $\tilde{\mathcal{V}}$ tiene los siguientes puntos críticos:

Proposición 60 Si $3125 \alpha^{4} \beta^{4}-4 \beta>0$ la función potencial (6.3) no tiene puntos críticos. Si $3125 \alpha^{4} \beta^{4}-4 \beta=0, \tilde{\mathcal{V}}$ tiene un punto crítico (en este caso obtenemos una raíz doble para la ecuación (6.4)). Si $3125 \alpha^{4} \beta^{4}-4 \beta<0$ y $\beta>0$ tenemos dos puntos críticos. Si $\beta<0 \tilde{\mathcal{V}}$ tenemos un sólo un punto crítico (en este caso obtenemos una raíz simple de la ecuación (6.4)). Y por último si $\beta=0$ tenemos un único punto crítico.

Observación 61 Nótese que si $\beta=0$ el potencial ampliado es

$$
\tilde{\mathcal{V}}(r, \theta)=-\frac{\alpha^{2} r^{2}}{2}-\frac{1}{r}
$$

y el término $\frac{\beta}{r^{3}}$ asociado al efecto de la forma del cuerpo celeste desaparece. En este caso, obtenemos el hamiltoniano de Kepler en un sistema de referencia rotante.

Sea $\pi: \mathbf{E} \longrightarrow \mathbb{R}^{+} \times S^{1}$ la proyección natural del espacio de fases $\mathbf{E}$ en el espacio de configuración $\mathbb{R}^{+} \times S^{1}$. Para cada $h \in \mathbb{R}$ la región de Hill $R_{h}$ está definida por $R_{h}=\pi\left(E_{h}\right)$.

$$
R_{h}=\left\{(r, \theta) \in \mathbb{R}^{+} \times S^{1}: \widetilde{\mathcal{V}} \leq h\right\}
$$

Se puede ver que

$$
R_{h} \approx\left\{r \in \mathbb{R}^{+}:-\frac{\alpha^{2} r^{2}}{2}-\frac{1}{r}+\frac{\beta}{r^{3}} \leq h\right\} \times S^{1}
$$

donde $\approx$ denota el difeomorfismo entre dos variadades diferenciables.

Las superficies de velocidad nula $S_{h}$ están determinadas por

$$
S_{h}=\left\{r \in \mathbb{R}^{+}:-\frac{\alpha^{2} r^{2}}{2}-\frac{1}{r}+\frac{\beta}{r^{2}}=h\right\} \times S^{1}
$$

\subsection{Topología de la regiones de Hill}

En lo que sigue denotaremos por $h_{i}=\widetilde{\mathcal{V}}\left(r_{i}\right)(i=1,2,3,4)$ (donde $r_{i}(i=1,2,3,4)$ son las raíces del polinomio (6.4) respecto de $r$ ) a los puntos críticos del potencial. Y por $A_{i}(i=1,2,3,4)$ a los puntos de corte de la gráfica del potencial ampliado con $\widetilde{\mathcal{V}}=h$.

Para poder entender mejor la topología de las regiones de Hill introducimos las siguientes figuras 


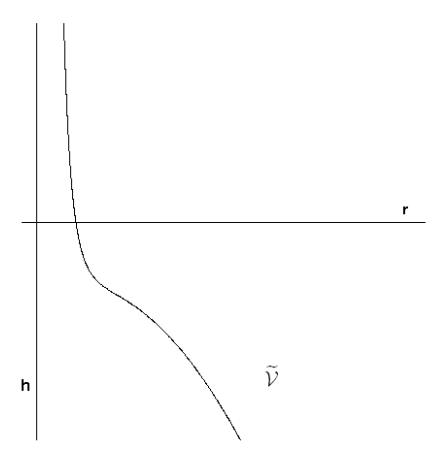

Figura 6.1: Gráfica del potencial ampliado, para $3125 \alpha^{4} \beta^{4}-4 \beta>0$ (caso en el que no hay puntos críticos).

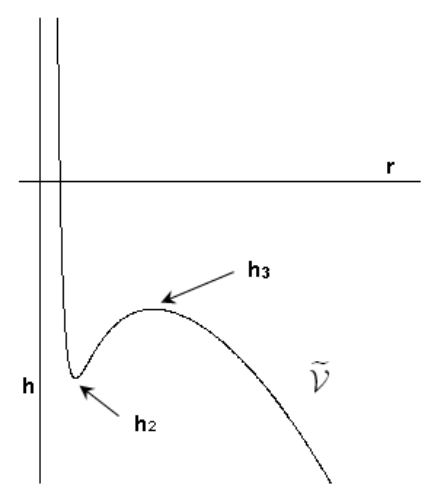

Figura 6.3: Gráfica de la función potencial ampliado para $3125 \alpha^{4} \beta^{4}-4 \beta<0 \mathrm{y}$ $\beta>0$ (caso en el que tenemos dos puntos críticos).

Empleando los resultados anteriores se obtiene la siguiente clasificación topológica de las regiones de Hill $R_{h}$. Ésta dependerá de los parámetros $\alpha, \beta$ y de las relaciones existentes entre ambos.

\begin{tabular}{|l|l|l|}
\hline $3125 \alpha^{4} \beta^{4}-4 \beta>0$ & $\forall h$ & $R_{h} \approx\left[A_{1},+\infty\right) \times S^{1}$ \\
\hline
\end{tabular}

Tabla 6.1: Clasificación topológica de las regiones de Hill para $3125 \alpha^{4} \beta^{4}-4 \beta>0$ (caso en el que no hay puntos críticos). Corresponde a la figura (6.1).
Figura 6.2: Gráfica de la función potencial ampliado para $3125 \alpha^{4} \beta^{4}-4 \beta=0$ (caso en el que hay un solo punto crítico, en este tenemos una raíz doble).

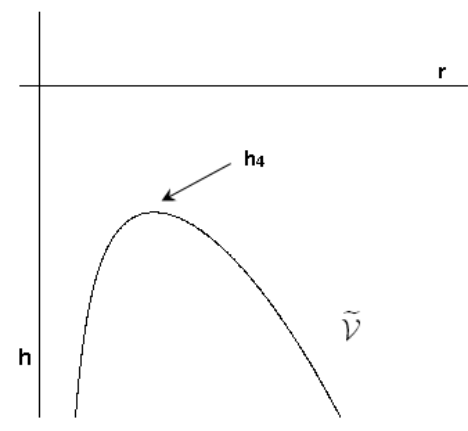

Figura 6.4: Gráfica de la función potencial para $\beta<0$ (caso en el que un único punto crítico, en este caso tenemos una raíz simple). 


\begin{tabular}{|l|l|l|}
\hline \multirow{3}{*}{$3125 \alpha^{4} \beta^{4}-4 \beta=0$} & $h<h_{1}$ & $R_{h} \approx\left[A_{1},+\infty\right) \times S^{1}$ \\
\cline { 2 - 3 } & $h=h_{1}$ & $R_{h} \approx\left[h_{1},+\infty\right) \times S^{1}$ \\
\cline { 2 - 3 } & $h>h_{1}$ & $R_{h} \approx\left[A_{1},+\infty\right) \times S^{1}$ \\
\hline
\end{tabular}

Tabla 6.2: Clasificación topológica de las regiones de Hill para $3125 \alpha^{4} \beta^{4}-4 \beta=0$ (caso en el que hay un solo punto crítico, en este tenemos una raíz doble). Corresponde a la figura (6.2).

\begin{tabular}{|l|l|l|}
\hline \multirow{3}{*}{$\beta>0$ y } & $h<h_{2}$ & $R_{h} \approx\left[A_{1},+\infty\right) \times S^{1}$ \\
\cline { 2 - 3 } $3125 \alpha^{4} \beta^{4}-4 \beta<0$ & $h=h_{2}$ & $R_{h} \approx\left\{\left\{h_{2}\right\} \cup\left[A_{4},+\infty\right)\right\} \times S^{1}$ \\
\cline { 2 - 3 } & $h_{2}<h<h_{3}$ & $R_{h} \approx\left[\left[A_{1}, A_{2}\right] \cup\left[A_{3},+\infty\right)\right] \times S^{1}$ \\
\cline { 2 - 3 } & $h=h_{3}$ & $R_{h} \approx\left[\left[A_{1}, h_{3}\right] \cup\left[h_{3},+\infty\right)\right] \times S^{1}$ \\
\cline { 2 - 3 } & $h>h_{3}$ & $R_{h} \approx\left[A_{1},+\infty\right) \times S^{1}$ \\
\hline
\end{tabular}

Tabla 6.3: Clasificación topológica de las regiones de Hill para $3125 \alpha^{4} \beta^{4}-4 \beta<0$ y $\beta>0$ (caso en el que tenemos dos puntos críticos). Corresponde a la figura (6.3).

\begin{tabular}{|l|l|l|}
\hline \multirow{3}{*}{$\beta<0$} & $h<h_{4}$ & $R_{h} \approx\left\{\left(0, A_{1}\right] \cup\left[A_{2},+\infty\right)\right\} \times S^{1}$ \\
\cline { 2 - 3 } & $h=h_{4}$ & $R_{h} \approx\left\{\left(0, h_{4}\right] \cup\left[h_{4},+\infty\right)\right\} \times S^{1}$ \\
\cline { 2 - 3 } & $h>h_{4}$ & $R_{h} \approx(0,+\infty) \times S^{1}$ \\
\hline
\end{tabular}

Tabla 6.4: Clasificación topológica de las regiones de Hill para $\beta<0$ (caso en el que un único punto crítico, en este caso tenemos una raíz simple). Corresponde a la figura (6.4). 
Observación 62 Para el caso en que $\beta=0$ estamos en el caso kepleriano.

\subsection{Estudio cualitativo del flujo hamiltoniano}

En esta sección estudiamos la topología de las variedades invariantes $\mathcal{H}^{-1}(h)=E_{h}$ e $I_{h k}$. Con idea de dar una clasificación topológica a dichas variedades necesitamos nuevos resultados y recordar la siguiente notación:

Recordemos que los conjuntos $E_{h}$ e $I_{h k}$ venían dados por

$$
\begin{gathered}
E_{h}=\left\{\left(r, \theta, p_{r}, p_{\theta}\right) \in \mathbf{E}: \mathcal{H}\left(r, \theta, p_{r}, p_{\theta}\right)=h\right\}, h \in \mathbb{R}, \\
J_{k}=\left\{\left(r, \theta, p_{r}, p_{\theta}\right) \in \mathbf{E}: p_{\theta}=k\right\}, k \in \mathbb{R}, \\
I_{h k}=E_{h} \cap J_{k},
\end{gathered}
$$

$\operatorname{con} \mathbf{z}=\left(r, \theta, p_{r}, p_{\theta}\right) \in \mathbf{E} \mathrm{y}(h, k) \in \mathbb{R}^{2}$.

Definimos ahora una nueva aplicación $g: \mathbb{R}^{+} \times \mathbb{R}^{2} \longrightarrow \mathbb{R}$, con $g\left(r, p_{r}, p_{\theta}\right)=\mathcal{H}\left(r, \theta, p_{r}, p_{\theta}\right)$, como

$$
g\left(r, p_{r}, p_{\theta}\right)=\frac{1}{2}\left(p_{r}^{2}+\frac{p_{\theta}^{2}}{r^{2}}\right)+\alpha p_{\theta}-\frac{1}{r}+\frac{b}{r^{3}} .
$$

Si $h \in \mathbb{R}$ es un valor regular de la aplicación $g: \mathbb{R}^{+} \times \mathbb{R}^{2} \longrightarrow \mathbb{R}$ y $g^{-1}(h) \neq \emptyset, g^{-1}(h)$ es una superficie de $\mathbb{R}^{+} \times \mathbb{R}^{2}$ que llamaremos superficie de energía.

Observación 63 Observemos que $g^{-1}(h)=E_{h} / S^{1}$.

Por lo tanto, en términos de la superficie de energía, los conjuntos invariantes quedan definidos como siguen

$$
\begin{gathered}
E_{h}=\mathcal{H}^{-1}(h)=\left\{\left(r, \theta, p_{r}, p_{\theta}\right) \in \mathbf{E}: g\left(r, p_{r}, p_{\theta}\right)=h\right\} \approx g^{-1}(h) \times S^{1}, \\
J_{k}=\left\{z \in \mathbf{E}: p_{\theta}=k\right\}, k \in \mathbb{R}, \\
I_{h k}=E_{h} \cap J_{k} \approx\left(g^{-1}(h) \cap\left\{p_{\theta}=k\right\}\right) \times S^{1},
\end{gathered}
$$

$\operatorname{con} \mathbf{z}=\left(r, \theta, p_{r}, p_{\theta}\right) \in \mathbf{E} \mathrm{y}(h, k) \in \mathbb{R}^{2}$.

Para el estudio de la topología de $\mathcal{H}^{-1}(h)=E_{h}$ y de $I_{h k},(h, k) \in \mathbb{R}^{2}$ debemos caracterizar primero los puntos de equilibrio del hamiltoniano $\mathcal{H}$, ya que las superficies de energía son regulares cuando $h \neq h_{i}$, siendo $h_{i}=\mathcal{H}\left(z_{e_{i}}\right)$ donde $z_{e_{i}}$ se corresponde con cada uno de los puntos de equilibrio del hamiltoniano $\mathcal{H}$. Y por último debemos calcular los extremos de la superficie de energía $g^{-1}(h)$.

Mediante un cálculo estándar se puede verificar el siguiente resultado:

Lema $64 \mathbf{z}_{e}=\left(r_{e}, \theta_{e}, p_{r_{e}}, p_{\theta_{e}}\right) \in \mathbf{E}$ es un equilibrio del flujo hamiltoniano

$$
\begin{aligned}
\frac{d r}{d t} & =\frac{d \mathcal{H}}{d p_{r}}, \quad \frac{d \theta}{d t}=\frac{d H}{d p_{\theta}} \\
\frac{d p_{r}}{d t} & =-\frac{d H}{d r}, \quad \frac{d p_{\theta}}{d t}=-\frac{d H}{d \theta}
\end{aligned}
$$


si y sólo si $\tilde{\mathbf{z}_{e}}=\left(r_{e}, \theta_{e}\right)$ es punto crítico de

$$
\tilde{\mathcal{V}}=-\frac{\alpha^{2} r^{2}}{2}-\frac{1}{r}+\frac{\beta}{r^{3}}
$$

Observación $65 \pi\left(\tilde{\mathbf{z}_{e}}\right)=\mathbf{z}_{e}$, donde $\pi: \mathbf{E} \longrightarrow \mathbb{R}^{+} \times S^{1}$ es la proyección natural.

Utilizando el algoritmo de Sturm para saber el número de raíces reales de la ecuación:

$$
\alpha^{2} r^{5}-r^{2}+3 \beta=0
$$

Obtenemos el siguiente resultado:

Proposición 66 Si $3125 \alpha^{4} \beta^{4}-4 \beta>0, \mathcal{H}$ no tiene familias de puntos de equilibrio. Si $3125 \alpha^{4} \beta^{4}-4 \beta=0, \mathcal{H}$ tiene una familia de puntos de equilibrio (hay una raiz doble). Si $3125 \alpha^{4} \beta^{4}-4 \beta<0$ y $\beta>0, \mathcal{H}$ tiene dos familias de puntos de equilibrio. Si $\beta<0, \mathcal{H}$ tiene una familia de puntos de equilibrio (hay una raíz simple). Si $\beta=0$, nuestro hamiltoniano tiene una única familia de puntos de equilibrio.

En lo que sigue, los valores de la energía en cada una de las familias de equilibrios serán denotados por $h_{i}=\mathcal{H}\left(r_{i}, \theta, 0, p_{\theta_{i}}\right),(i=1,2,3,4)$.

Observación 67 Por el lema anterior podemos afirmar que los valores $h_{i}=\mathcal{H}\left(r_{i}, \theta, 0, p_{\theta_{i}}\right)$, $i=1,2,3,4$, son los mismos que los $h_{i}$ utilizados en el estudio de las regiones de Hill.

La proposición anterior caracteriza completamente la topología de $\mathcal{H}^{-1}(h)=E_{h}$.

\subsubsection{Trayectorias acotadas}

Despejando $p_{r}^{2}$ en hamiltoniano $\mathcal{H}$ obtenemos el siguiente polinomio

$$
-2 \beta-k^{2} r+2 r^{2}-2(h-\alpha k) r^{3}
$$

Del cual, utilizando el algoritmo de Sturm, podemos calcular el número de raíces reales positivas del polinomio con respecto a $r$ y las zonas donde las trayectorias de la superficie de energía están acotadas o no.

Proposición 68 Las regiones donde la superficie tiene trayectorias acotadas son

$\mathbf{1}^{a}$ Región Caso en que $\beta>0$. Las trayectorias serán acotadas en la región que delimitan las siguientes desigualdades (y además teniendo en cuenta las relaciones existentes entre ambos parámetros: $3125 \alpha^{4} \beta^{4}-4 \beta>0,3125 \alpha^{4} \beta^{4}-4 \beta=0$ ó $3125 \alpha^{4} \beta^{4}-4 \beta<$ $0)$ :

$$
\begin{aligned}
& \beta>0 \\
& h-\alpha k<0 \\
& 108 \beta^{2}(h-\alpha k)^{2}+k^{4}\left(-1-2 h k^{2}+2 \alpha k^{3}\right)-4 \beta\left(4+9 k^{2}(h-\alpha k)\right)<0
\end{aligned}
$$

$\mathbf{2}^{a}$ Región Caso en que $\beta<0$. No existen trayectorias acotadas.

$3^{a}$ Región Caso en que $\beta=0$. La ecuación $h-k=0$ nos da el valor $a_{1}$, a partir del cual todas las trayectorias no están acotadas (véase capitulo 5). 
Para cada uno de los casos pintemos las diferentes regiones. En estas figuras la zona azul es la región donde sabemos que las trayectorias están acotadas.

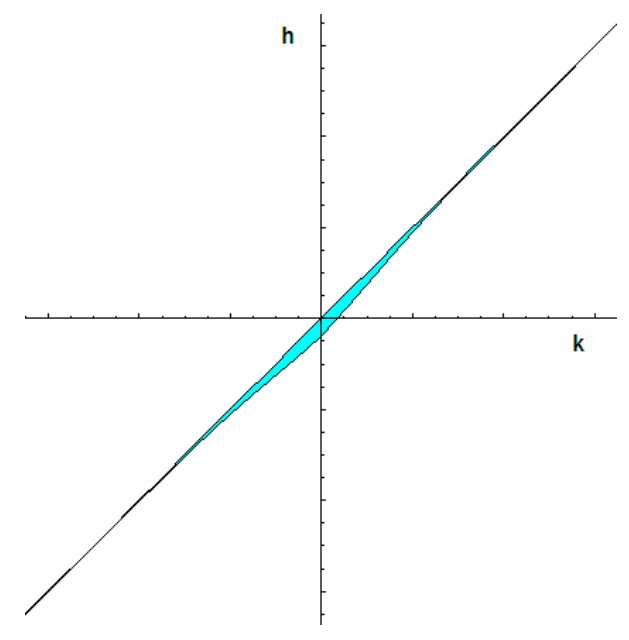

Figura 6.5: Región delimitada por las desigualdades (6.10) y con la condición $3125 \alpha^{4} \beta^{4}-4 \beta>0$ (caso en el que no hay puntos críticos).

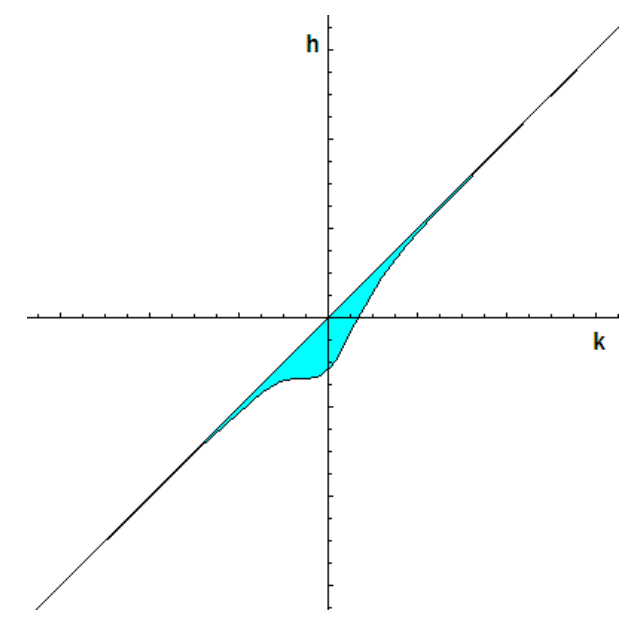

Figura 6.6: Región delimitada por las desigualdades (6.10) y con la condición $3125 \alpha^{4} \beta^{4}-4 \beta<0$ y $\beta>0$ (caso en el que tenemos dos puntos críticos).

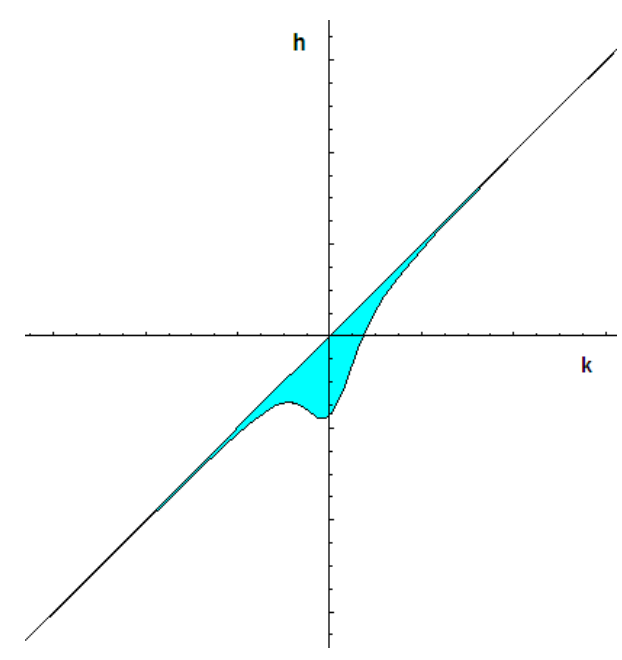

Figura 6.7: Región delimitada por las desigualdades (6.10) y con la condición $3125 \alpha^{4} \beta^{4}-4 \beta<0$ y $\beta>0$ (caso en el que tenemos dos puntos críticos).

Observación 69 El caso en que $\beta<0$ no se dibuja, ya que, como hemos comentado anteriormente, no posee trayectorias acotadas. El caso en que $\beta=0$ corresponde al caso kepleriano. 


\subsubsection{Clasificación topológica de $E_{h}$ e $I_{h k}$}

En esta parte del capítulo estudiaremos la topología de las variedades invariantes $\mathcal{H}^{-1}(h)=E_{h}$ e $I_{h k}$. Para ello introducimos toda aquella notación que es necesaria para poder entender las distintas topologías.

Sea $S^{n-1}$ la esfera en $\mathbb{R}^{n}$, con $n>1$. $Y$ es la unión de dos toros sólidos abiertos que se identifican punto a punto con los puntos de dos círculos de cada toro, que no pueden ser contraidos a un solo punto dentro del correspondiente toro (véase Llibre (2001)[36] para más detalles).

Los valores $c_{j}, i=1,2,3$ son las raíces reales respecto de $k$ del polinomio

$$
18 \beta k^{2}+k^{6}-108 \beta^{2}(h-\alpha k)-12 \beta \sqrt{12 \beta+k^{4}}-k^{4} \sqrt{12 \beta+k^{4}}=0 .
$$

y corresponden a los extremos de la superficie que denotamos por $Q_{j},(j=1,2,3)$.

El valor $a_{1}$ es la raíz real respecto de $k$ del polinomio (6.9) y su valor depende de los valores de $\alpha$, de $\beta$ y de las relaciones que existentes entre ellos. A partir de este valor las trayectorias no estarán acotadas.

Finalmente $h_{i}=\mathcal{H}\left(r_{i}, \theta_{i}, 0, p_{\theta_{i}}\right)(i=1,2,3,4)$, son los puntos de equilibrio del hamiltoniano $\mathcal{H}$.

Obtenemos la siguiente clasificación topológica para $E_{h}$ e $I_{h k}$ que entenderemos mejor a través de las siguientes superficies y de sus correspondientes tablas.

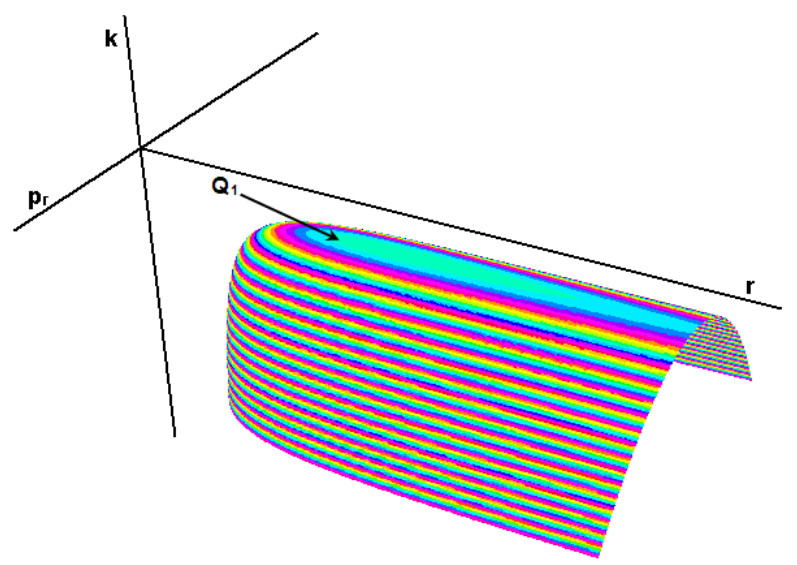

Figura 6.8: $g^{-1}(h)=E_{h} / S^{1}, \forall h$, cuando $3125 \alpha^{4} \beta^{4}-4 \beta>0$, es decir, cuando no hay familias de puntos de equilibrio. Donde $c_{1}$ es un extremo de la superficie de energía y $k=p_{\theta}$. 


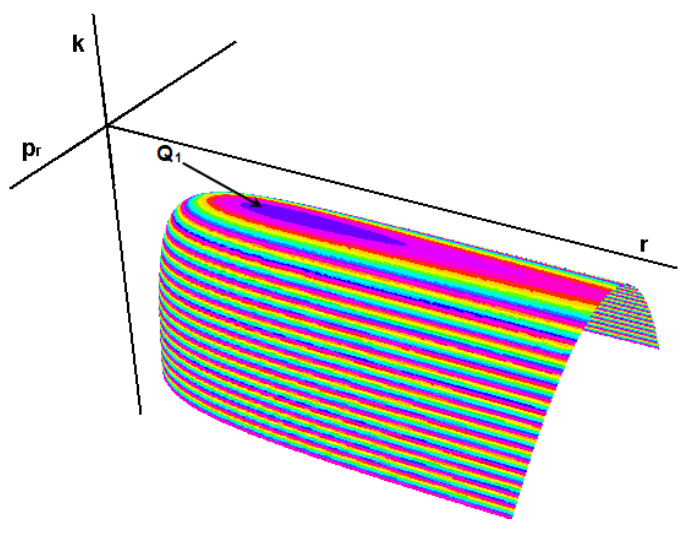

Figura 6.9: $g^{-1}(h)=E_{h} / S^{1}, h<h_{1}$, cuando $3125 \alpha^{4} \beta^{4}-4 \beta=0$, es decir, cuando hay una sola familia de puntos de equilibrio, en este tenemos una raíz doble. Donde $c_{1}$ es un extremo de la superficie de energía y $k=p_{\theta}$.

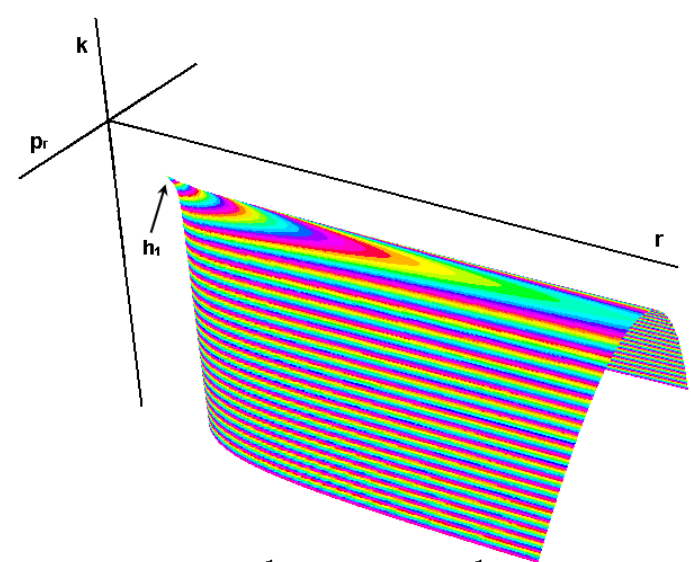

Figura 6.10: $g^{-1}(h)=E_{h} / S^{1}, h=h_{1}$, cuando $3125 \alpha^{4} \beta^{4}-4 \beta=0$, es decir, cuando hay una sola familia de puntos de equilibrio, en este caso tenemos una raíz doble. Donde $h_{1}$ es el punto de equilibrio y $k=p_{\theta}$.

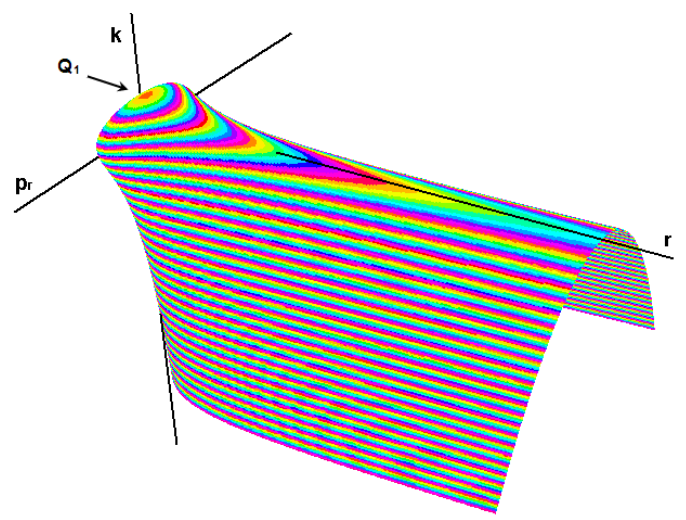

Figura 6.11: $g^{-1}(h)=E_{h} / S^{1}, h>h_{1}$, cuando $3125 \alpha^{4} \beta^{4}-4 \beta=0$, es decir, cuando hay una sola familia de puntos de equilibrio, en este tenemos una raíz doble. Donde $c_{1}$ es un extremo de la superficie de energía y $k=p_{\theta}$. 


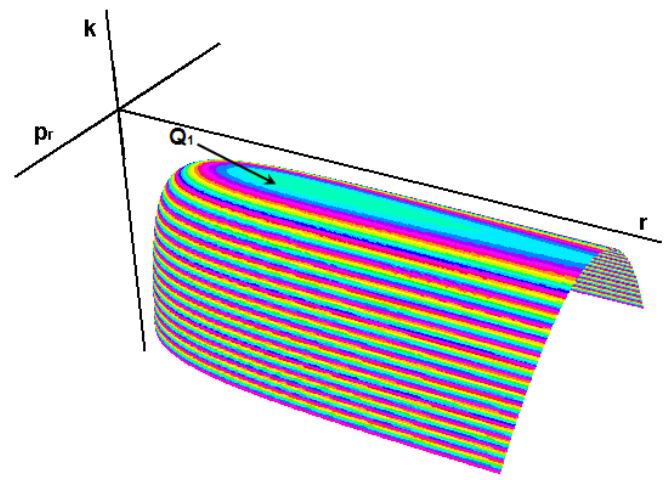

Figura 6.12: $g^{-1}(h)=E_{h} / S^{1}, h<h_{2}$, cuando $3125 \alpha^{4} \beta^{4}-4 \beta<0$, es decir, cuando hay cuando hay dos familias de puntos de equilibrio. Donde $c_{1}$ es un extremo de la superficie de energía y $k=p_{\theta}$.

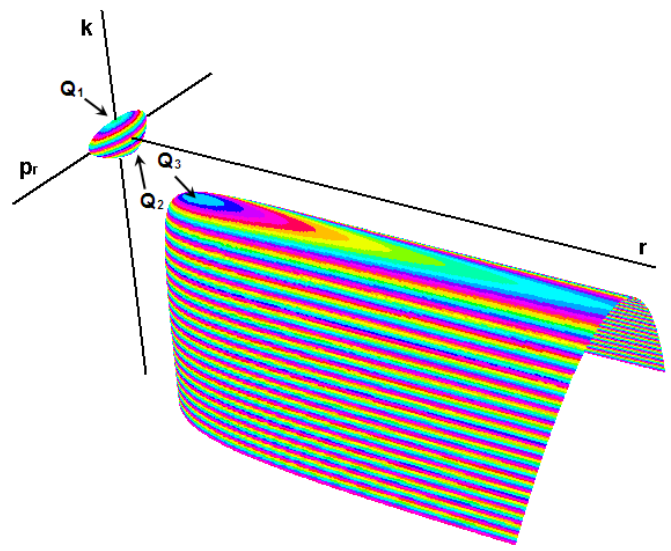

Figura 6.14: $g^{-1}(h)=E_{h} / S^{1}, h_{2}<h<$ $h_{3}$, cuando $3125 \alpha^{4} \beta^{4}-4 \beta<0$, es decir, cuando hay cuando hay dos familias de puntos de equilibrio. Donde $c_{1}, c_{2}$ y $c_{3}$ son tres extremos de la superficie de energía y $k=p_{\theta}$.

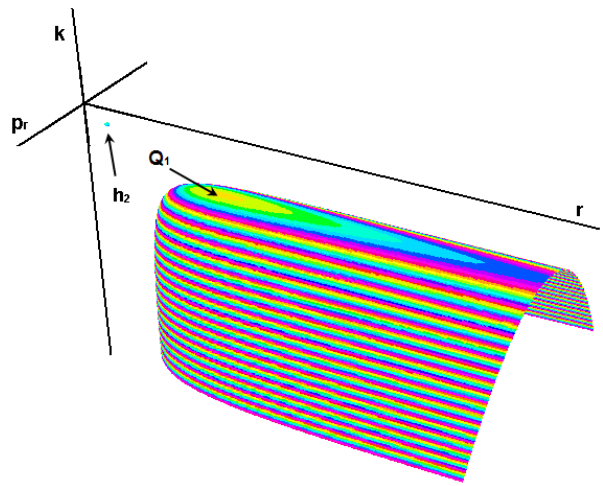

Figura 6.13: $g^{-1}(h)=E_{h} / S^{1}, h=h_{2}$, cuando $3125 \alpha^{4} \beta^{4}-4 \beta<0$, es decir, cuando hay cuando hay dos familias de puntos de equilibrio. Donde $c_{1}$ es un extremo de la superficie de energía, $h_{2}$ es un punto de equilibrio y $k=p_{\theta}$.

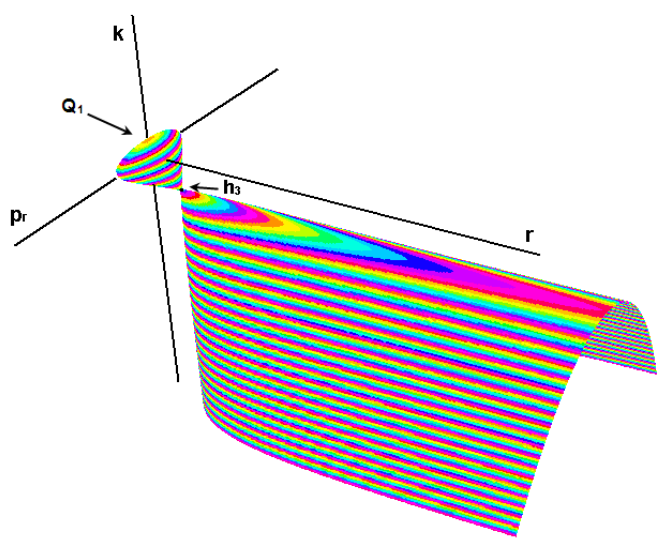

Figura 6.15: $g^{-1}(h)=E_{h} / S^{1}, h=h_{3}$, cuando $3125 \alpha^{4} \beta^{4}-4 \beta<0$, es decir, cuando hay cuando hay dos familias de puntos de equilibrio. Donde $c_{1}$ es un extremos de la superficie de energía, $h_{3}$ es un punto de equilibrio y $k=p_{\theta}$. 


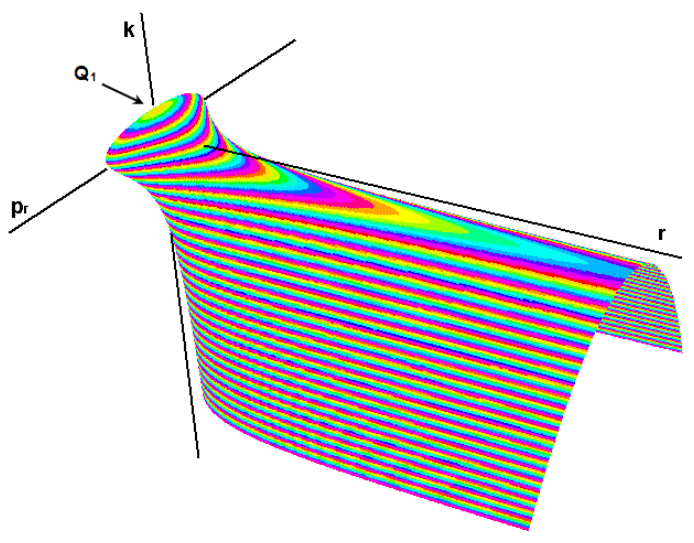

Figura 6.16: $g^{-1}(h)=E_{h} / S^{1}, h>h_{3}$, cuando $3125 \alpha^{4} \beta^{4}-4 \beta<0$, es decir, cuando hay cuando hay dos familias de puntos de equilibrio. Donde $c_{1}$ es un extremo de la superficie de energía y $k=p_{\theta}$.

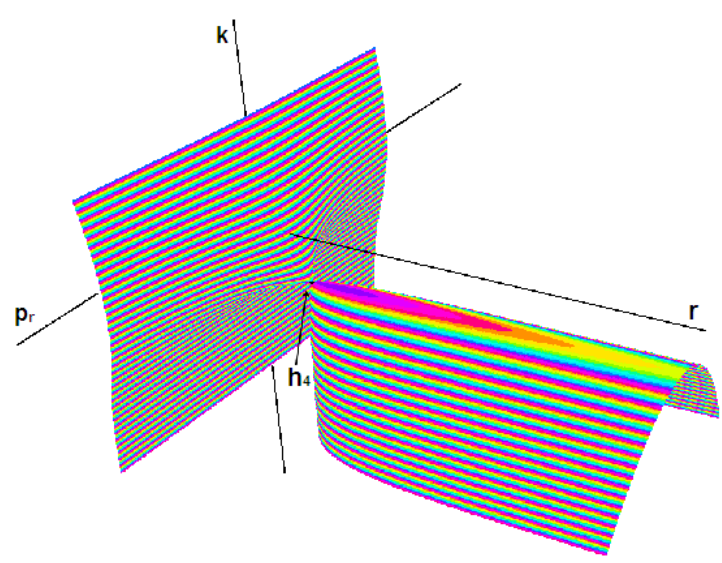

Figura 6.18: $g^{-1}(h)=E_{h} / S^{1}, h=h_{4}$, cuando $\beta<0$, es decir, cuando hay una única familia de puntos de equilibio, en este caso tenemos una raíz simple. Donde $c_{1}$ es un extremo de la superficie de energía, $h_{4}$ es un punto de equilibrio y $k=p_{\theta}$.

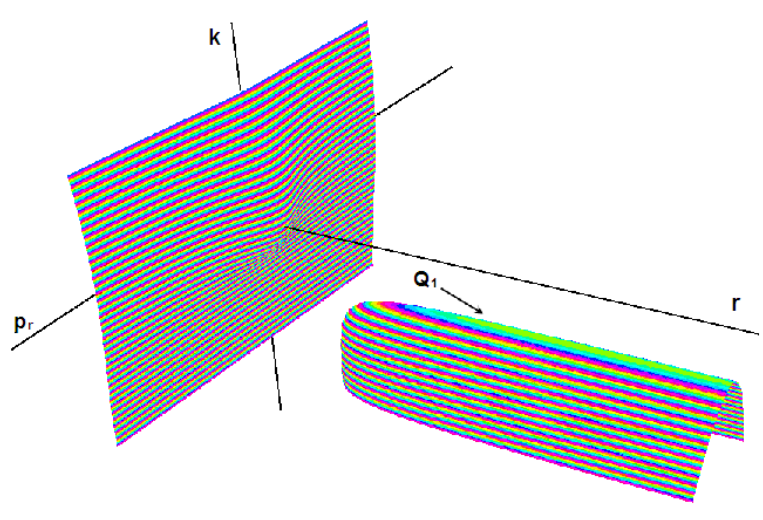

Figura 6.17: $g^{-1}(h)=E_{h} / S^{1}, h<h_{4}$, cuando $\beta<0$, es decir, cuando hay una única familia de puntos de equilibio, en este caso tenemos una raíz simple. Donde $c_{1}$ es un extremo de la superficie de energía y $k=p_{\theta}$.

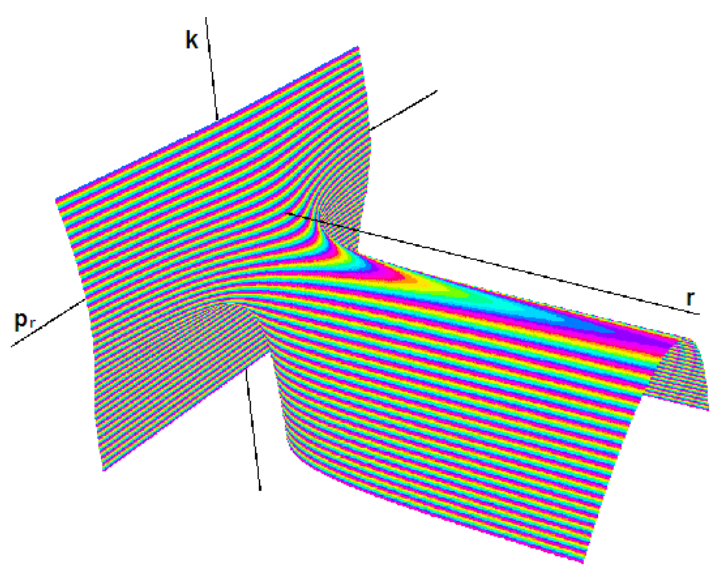

Figura 6.19: $g^{-1}(h)=E_{h} / S^{1}, h>h_{4}$, cuando $\beta<0$, es decir, cuando hay una única familia de puntos de equilibio, en este caso tenemos una raíz simple. Donde $k=p_{\theta}$. 


\begin{tabular}{|c|c|ll|}
\hline$h$ & $E_{h}$ & \multicolumn{2}{|c|}{$I_{h k}$} \\
\hline \multirow{4}{*}{$\forall h$} & \multirow{3}{*}{$S^{3} \backslash\left\{S^{1} \cup S^{1}\right\}$, véase 6.8} & $\emptyset$ & $k>c_{1}$ \\
& & $S^{1}$ & $k=c_{1}$ \\
& & $S^{1} \times S^{1}$ & $a_{1}<k<c_{1}$ \\
& & $S^{1} \times \mathbb{R}$ & $k \leq a_{1}$ \\
\hline
\end{tabular}

Tabla 6.5: Clasificación topológica de $E_{h}$ e $I_{h k}$ cuando $3125 \alpha^{4} \beta^{4}-4 \beta>0$, es decir, cuando no hay familias de puntos de equilibrio.

\begin{tabular}{|c|c|ll|}
\hline \multicolumn{1}{|c|}{$E_{h}$} & \multicolumn{2}{|c|}{$I_{h k}$} \\
\hline \multirow{5}{*}{$h<h_{1}$} & \multirow{3}{*}{$S^{3} \backslash\left\{S^{1} \cup S^{1}\right\}$, véase 6.9} & $\emptyset$ & $k>c_{1}$ \\
& & $S^{1} \times S^{1}$ & $a_{1}<k<c_{1}$ \\
& & $S^{1} \times \mathbb{R}$ & $k \leq a_{1}$ \\
\hline \multirow{5}{*}{$h=h_{1}$} & \multirow{3}{*}{$S^{3} \backslash\left\{S^{1} \cup S^{1}\right\}$, véase 6.10} & $\emptyset$ & $k>h_{1}$ \\
& & $S^{1} \times S^{1}$ & $a_{1}<k<h_{1}$ \\
& & $S^{1} \times \mathbb{R}$ & $k \leq a_{1}$ \\
\hline \multirow{5}{*}{$h>h_{1}$} & \multirow{3}{*}{$S^{3} \backslash\left\{S^{1} \cup S^{1}\right\}$, véase 6.11} & $\emptyset$ & $k>c_{1}$ \\
& & $S^{1}$ & $k=c_{1}$ \\
& & $S^{1} \times S^{1}$ & $a_{1}<k<c_{1}$ \\
& & $S^{1} \times \mathbb{R}$ & $k \leq a_{1}$ \\
\hline
\end{tabular}

Tabla 6.6: Clasificación topológica de $E_{h}$ e $I_{h k}$ cuando $3125 \alpha^{4} \beta^{4}-4 \beta=0$, es decir, cuando hay una sola familia de puntos de equilibrio. En este caso tenemos una raíz doble. 


\begin{tabular}{|c|c|c|c|}
\hline$h$ & $E_{h}$ & \multicolumn{2}{|l|}{$I_{h k}$} \\
\hline$h<h_{2}$ & $S^{3} \backslash\left\{S^{1} \cup S^{1}\right\}$, véase 6.12 & $\begin{array}{l}\emptyset \\
S^{1} \\
S^{1} \times S^{1} \\
S^{1} \times \mathbb{R}\end{array}$ & $\begin{array}{l}k>c_{1} \\
k=c_{1} \\
a_{1}<k<c_{1} \\
k \leq a_{1}\end{array}$ \\
\hline$h=h_{2}$ & $\left\{S^{1}\right\} \cup S^{3} \backslash\left\{S^{1} \cup S^{1}\right\}$, véase 6.13 & $\begin{array}{l}\emptyset \\
S^{1} \\
\emptyset \\
S^{1} \times S^{1} \\
S^{1} \times \mathbb{R}\end{array}$ & $\begin{array}{l}k>h_{2} \\
k=h_{2} \\
c_{1}<k<h_{2} \\
a_{1}<k<c_{1} \\
k \leq a_{1}\end{array}$ \\
\hline$h_{2}<h<h_{3}$ & $\left\{S^{3}\right\} \cup S^{3} \backslash\left\{S^{1} \cup S^{1}\right\}$, véase 6.14 & $\begin{array}{l}\emptyset \\
S^{1} \\
S^{1} \times S^{1} \\
S^{1} \\
\emptyset \\
S^{1} \\
S^{1} \times S^{1} \\
S^{1} \times \mathbb{R}\end{array}$ & $\begin{array}{l}k>c_{1} \\
k=c_{1} \\
c_{2}<k<c_{1} \\
k=c_{2} \\
c_{3}<k<c_{2} \\
k=c_{3} \\
a_{1}<k<c_{3} \\
k \leq a_{1}\end{array}$ \\
\hline$h=h_{3}$ & $\left\{S^{3}\right\} \cup S^{3} \backslash\left\{S^{1} \cup S^{1}\right\}$, véase 6.15 & $\begin{array}{l}\emptyset \\
S^{1} \\
S^{1} \times S^{1} \\
S^{1} \\
S^{1} \times S^{1} \\
S^{1} \times \mathbb{R}\end{array}$ & $\begin{array}{l}k>c_{1} \\
k=c_{1} \\
h_{3}<k<c_{1} \\
k=h_{3} \\
a_{1} \leq k<h_{3} \\
k \leq a_{1}\end{array}$ \\
\hline$h>h_{3}$ & $S^{3} \backslash\left\{S^{1} \cup S^{1}\right\}$, véase 6.16 & $\begin{array}{l}\emptyset \\
S^{1} \\
S^{1} \times S^{1} \\
S^{1} \times \mathbb{R}\end{array}$ & $\begin{array}{l}k>c_{1} \\
k=c_{1} \\
a_{1}<k<c_{1} \\
k \leq a_{1}\end{array}$ \\
\hline
\end{tabular}

Tabla 6.7: Clasificación topológica de $E_{h}$ e $I_{h k}$ cuando $3125 \alpha^{4} \beta^{4}-4 \beta<0$ y $\beta>0$, es decir, cuando hay dos familias de puntos críticos. 


\begin{tabular}{|c|c|c|c|}
\hline$h$ & $E_{h}$ & $I_{h k}$ & \\
\hline$h<h_{4}$ & $\left\{S^{3} \backslash S^{1}\right\} \cup\left\{S^{3} \backslash S^{1}\right\}$, véase 6.17 & $\begin{array}{l}S^{1} \times \mathbb{R} \\
\left\{S^{1} \times \mathbb{R}\right\} \cup S^{1} \\
\left\{S^{1} \times \mathbb{R}\right\} \cup\left\{S^{1} \times S^{1}\right\} \\
\left\{S^{1} \times \mathbb{R}\right\} \cup\left\{S^{1} \times \mathbb{R}\right\}\end{array}$ & $\begin{array}{l}k>c_{1} \\
k=c_{1} \\
a_{1}<k<c_{1} \\
k \leq a_{1}\end{array}$ \\
\hline$h=h_{4}$ & $Y$, véase 6.18 & $\begin{array}{l}S^{1} \times \mathbb{R} \\
\left\{S^{1} \times \mathbb{R}\right\} \cup\left\{S^{1} \times S^{1}\right\} \\
\left\{S^{1} \times \mathbb{R}\right\} \cup\left\{S^{1} \times \mathbb{R}\right\}\end{array}$ & $\begin{array}{l}k>c_{1} \\
a_{1}<k \leq c_{1} \\
k \leq a_{1}\end{array}$ \\
\hline$h>h_{4}$ & $S^{3} \backslash\left\{S^{1} \cup S^{1}\right\}$, véase 6.19 & $S^{1} \times \mathbb{R}$ & $\forall k$ \\
\hline
\end{tabular}

Tabla 6.8: Tabla: Clasificación topológica de $E_{h}$ e $I_{h k}$ cuando $\beta<0$, es decir, cuando tenemos una única familia de puntos de equilibrio. En este caso tenemos una raíz simple.

\subsection{Conclusiones}

En este capítulo se ha considerado una aproximación al Main Problem del satélite artificial, que viene a ser el problema de Kepler al que se ha añadido una perturbación. A través de diferentes técnicas y del teorema de Liouville-Arnold, hemos podido realizar el estudio cualitativo de este sistema hamiltoniano. Gracias al cálculo de los puntos críiticos del potencial ampliado, obtenido mediante complección de cuadrados, hemos podido definir y calcular las regiones de Hill, regiones del espacio fásico donde existe movimiento real, así como su topología. También se han calculado las regiones donde las trayectorias son acotadas. Por último, tras definir lo que hemos llamado superficie de energía, se ha hecho la clasificación topológica de los subconjuntos invariantes $E_{h}$ e $I_{h k}$. 



\section{Introducción}

A continuación, podemos encontrar los algoritmos utilizados para el cálculo de los puntos críticos, de los extremos relativos de la superficie, para el estudio de la topología de $I_{h k}$ y los necesarios para hacer el estudio numérico de las órbitas. Estos se han realizado mediante el programa Mathematica para el caso del hamiltoniano del capítulo 4. Algoritmos similares, se han usado para el estudio de los hamiltonianos de los dos capítulos siguientes, pero con la idea de no ser repetitivos, solo mostraremos los concernientes a este caso.

En lo que sigue, y por comodidad, vamos a denotar en nuestro código a $p_{r}$ como $p_{1}$ y a $p_{\theta}$ como $p_{2}$.

Llamaremos $c_{i},(i=1,2,3,4)$ a los máximos y mínimos de las superficies de energía, $h_{i},(i=1,2,3)$ a los valores de $\mathcal{H}$ en los puntos críticos y como $a_{i},(i=1,2)$ a las raíces del polinomio en $k$

$$
h-\alpha(\beta-k)^{2}
$$





\section{Apéndice A}

\section{Cálculo de los equilibrios de $\mathcal{H}$}

Vamos a calcular los equilibrios del sistema de ecuaciones hamiltoniano (4.15). Para ello usaremos el siguiente código:

Hamiltoniano $:=1 / 2 \star\left(p 1^{\wedge} 2+p^{\wedge} 2 / \mathrm{r}^{\wedge} 2\right)+$ alpha * (beta $\left.-\mathrm{p} 2\right)^{\wedge} 2-1 / \mathrm{r}$

$\partial_{x}$ Hamiltoniano

Parcialr $:=-\frac{p 2^{2}}{\mathbf{r}^{3}}+\frac{1}{\mathbf{r}^{2}}$

$\partial_{\mathrm{p}^{2}}$ Hamiltoniano

Parcialp2 : = -2 alpha (beta - p2) $+\frac{\text { p2 }}{\mathbf{r}^{2}}$

Solve [Parcialp2 $=\mathbf{0}$, p2]

$\partial_{\mathrm{pl}}$ Hamiltoniano

Parcialp1 : = p1

Parcialr $/ .\left\{p 2 \rightarrow \frac{2 \text { alpha beta } r^{2}}{1+2 \text { alpha } r^{2}}, \mathbf{p} 1 \rightarrow 0\right\}$

Together $\left[\frac{1}{r^{2}}-\frac{4 \text { alpha }^{2} \text { beta } a^{2} r}{\left(1+2 \text { alpha } r^{2}\right)^{2}}\right]$

$p[1]:=1+4$ alpha $r^{2}-4$ alpha $^{2}$ beta ${ }^{2} r^{3}+4$ alpha $^{2} r^{4}$

Para obtener los puntos críticos del sistema debemos conocer el número de raíces reales del polinomio

$$
p[1]=1+4 \alpha r^{2}-4 \alpha^{2} \beta^{2} r^{3}+4 \alpha^{2} r^{4}
$$

Para ello, vamos a utilizar el Método de Sturm para el cálculo de raíces reales de este polinomio.

El método de Sturm es un algoritmo que se utiliza para buscar los ceros de un polinomio $p(x)$ y que nos da el número de raíces reales de dicho polinomio en un intervalo determinado. Para nosotros es una herramienta de gran interés, ya que permitirá el cálculo de los puntos críticos. Pero para ello antes debemos de definir lo que son los sistemas de polinomios de Sturm.

Supongamos que se van a buscar los ceros de un polinomio $p(x)$, lo primero que se tiene que buscar para poder utilizar el Teorema de Sturm es un sistema de polinomios especial para poder evaluar en él. Veamos como calcular éste sistema.

El primer polinomio $p_{1}(x)$ es el propio $p(x)$; el segundo polinomio, $p_{2}(x)$ es $\frac{d p_{1}(x)}{d x}$; para el tercer polinomio se debe realizar la división de $p_{1}(x)$ entre $p_{2}(x)$, el residuo que 
se obtiene con signo contrario será nuestro $p_{3}(x)$; este proceso se continúa, es decir $p_{4}(x)$ será el residuo con signo contrario de la división de $p_{2}(x)$ entre $p_{3}(x)$; el proceso acaba cuando se obtiene una constante.

Teorema 70 Sea $p(x)$ un polinomio de coeficientes reales tal que $p(x)=0$ no tiene raíces múltiples y constuyamos el sistema de Sturm para $p(x)$. Sean a y $b$ dos números reales con $a<b$, tales que ninguno sea raíz de $p(x)$. Entonces la cantidad de raíces reales de $p(x)=0$ entre a y $b$ es la diferencia entre el número de variaciones de signo del sistema de Sturm

$$
p_{1}(x), p_{2}(x), p_{3}(x), p_{4}(x), \ldots, p_{k-1}(x), p_{k}(x)
$$

para $x=b$ y el número de variaciones para $x=a$. Los términos que den cero deben ser descartados antes de contar los cambios de signo.

Observación 71 Nótese que el teorema pide que la ecuación $p(x)=0$ no tenga raíces múltiples, aún así el teorema funciona bien para muchos polinomios con raíces múltiples, este sería el caso de nuestro polinomio (A.1). Es más, son muy pocos los polinomios con raíces múltiples que fallan.

Definición 72 Denotamos por $V(a)=\left(p_{1}(a), p_{2}(a), p_{3}(a), p_{4}(a), \ldots, p_{k-1}(a), p_{k}(a)\right)$ y por $V(b)=\left(p_{1}(b), p_{2}(b), p_{3}(b), p_{4}(b), \ldots, p_{k-1}(b), p_{k}(b)\right)$.

Así pues, tendremos que

$\mathrm{N}^{o}$ de raíces $=\mathrm{N}^{o}$ de cambios de signo de $V(a)-\mathrm{N}^{o}$ de cambios de signo de $V(b)$

Abusando de la notación también usaremos $V(a)$ como el número de cambios de signo de $V(a)$ y $V(b)$ como el número de cambios de signo de $V(b)$.

Continuando con el estudio de las raíces reales de nuestro polinomio $p[1]$, tomaremos como intervalo para aplicar el método de Sturm el $(0,+\infty)$ debido a que $r$ toma siempre valores reales positivos.

Observación 73 Obsérvese que para el estudio de los signos de la sucesión de polinomios de Sturm en $+\infty$, debemos considerar el signo de los coeficientes del término de mayor grado de $r$.

Para ello usaremos el siguiente código

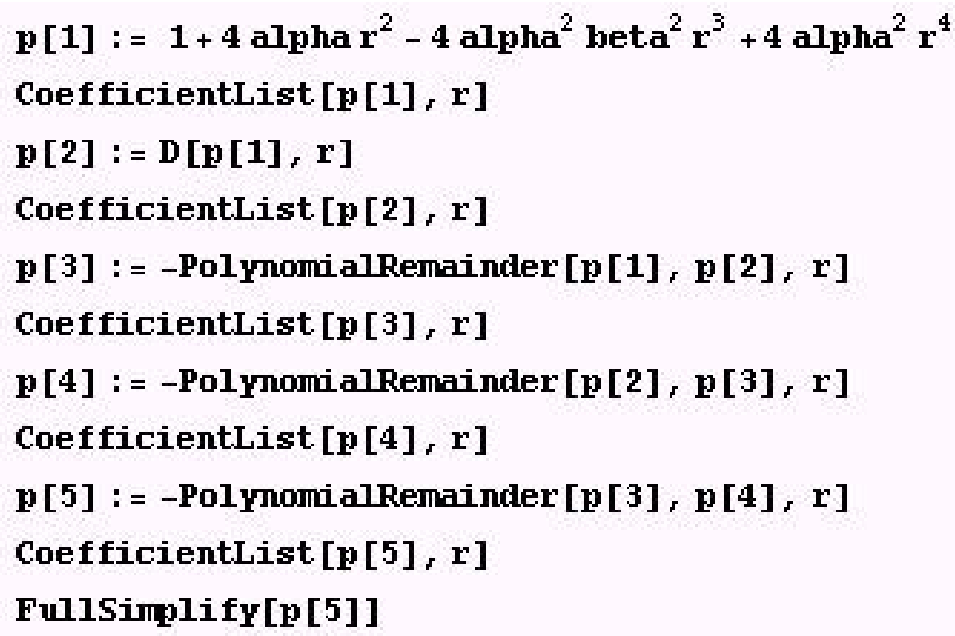


Donde

$V(0)=\left(1,0,-1,-32+9 \alpha \beta^{4},-128+27 \alpha \beta^{4}\right)=(+,+,-, \quad, \quad)$

$V(+\infty)=\left(4 \alpha^{2}, 16 \alpha^{2},-8+3 \alpha \beta^{4},-64 \alpha^{2} \beta^{4},-128+27 \alpha \beta^{4}\right)=(+,+, \quad,-, \quad)$

a) Para $27 \alpha \beta^{4}-128>0$ se tiene

$V(0)=(+,+,-,+,-)$, tenemos tres cambios de signo $V(+\infty)=(+,+,+,-,-)$, tenemos un cambio de signo

$\mathrm{N}^{o}$ de raíces $=V(0)-V(+\infty)=3-1=2$ raíces

b) Para $27 \alpha \beta^{4}-128=0$ obtenemos

$V(0)=(+,+,-,+, 0)$, tenemos dos cambios de signo

$V(+\infty)=(+,+,+,-, 0)$, tenemos un cambio de signo

$\mathrm{N}^{o}$ de raíces $=V(0)-V(+\infty)=2-1=1$ raíz

c) Para $27 \alpha \beta^{4}-128<0$ encontramos tres casos diferentes

- Para $-32+9 \alpha \beta^{4}>0$

$V(0)=(+,+,-,+,+)$, tenemos dos cambios de signo

$V(+\infty)=(+,+,+,-,+)$, tenemos dos cambios de signo

$\mathrm{N}^{o}$ de raíces $=V(0)-V(+\infty)=2-2=0$ raíces

- Para $-8+3 \alpha \beta^{4}>0 \mathrm{y}-32+9 \alpha \beta^{4}<0$

$V(0)=(+,+,-,-,+)$, tenemos dos cambios de signo

$V(+\infty)=(+,+,+,-,+)$, tenemos dos cambios de signo

$\mathrm{N}^{o}$ de raíces $=V(0)-V(+\infty)=2-2=0$ raíces 
- Para $-8+3 \alpha \beta^{4}<0$

$V(0)=(+,+,-,-,+)$, tenemos dos cambios de signo

$V(+\infty)=(+,+,-,-,+)$, tenemos dos cambios de signo

$$
\mathrm{N}^{o} \text { de raíces }=V(0)-V(+\infty)=2-2=0 \text { raíces }
$$

Resumiendo

\begin{tabular}{|l|l|}
\hline $27 \alpha \beta^{4}-128>0$ & 2 raíces \\
\hline $27 \alpha \beta^{4}-128=0$ & 1 raíz doble \\
\hline $27 \alpha \beta^{4}-128<0$ & 0 raíces \\
\hline
\end{tabular}

Tabla A.1: Resumen del número de raíces del polinomio (A.1). 


\section{Apéndice B}

\section{Extremos relativos de la superficie}

Para calcular los extremos relativos de la superficie, se ha utilizado el teorema de la Función Implícita. El código usado es el siguiente

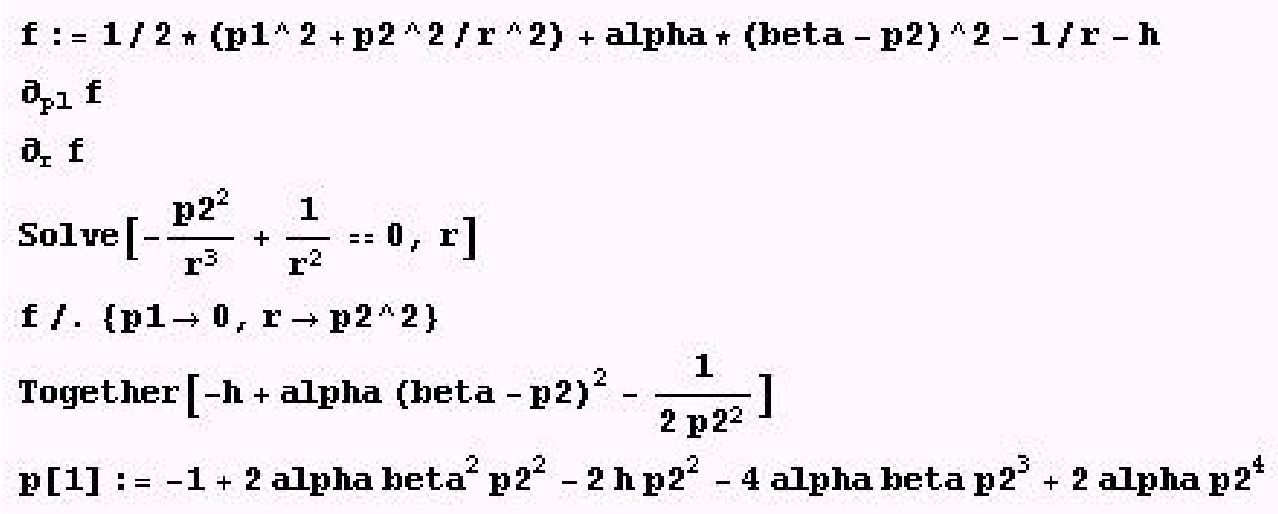

El cálculo de las raíces de la ecuación

$$
2 \alpha p_{\theta}^{4}-4 \alpha \beta p_{\theta}^{3}+\left(2 \alpha \beta^{2}-2 h\right) p_{\theta}^{2}-1=0
$$

ha sido llevado a cabo usando de nuevo el Método de Sturm.

El código usado es el siguiente

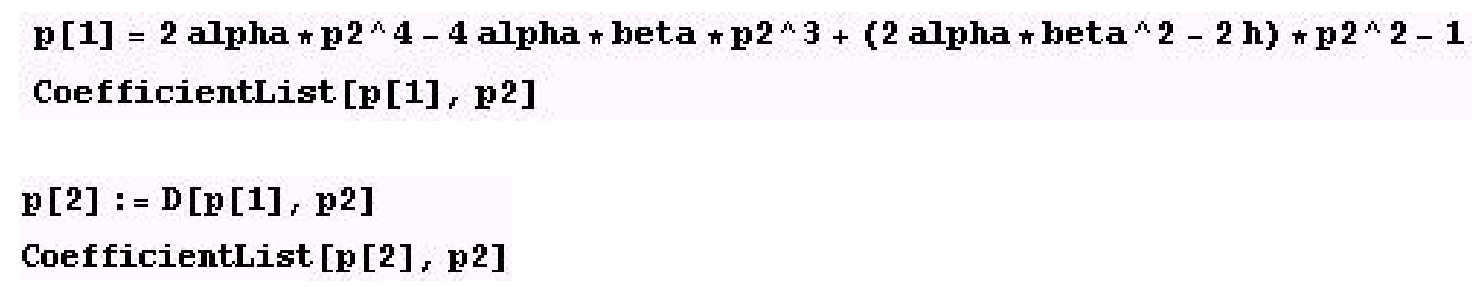




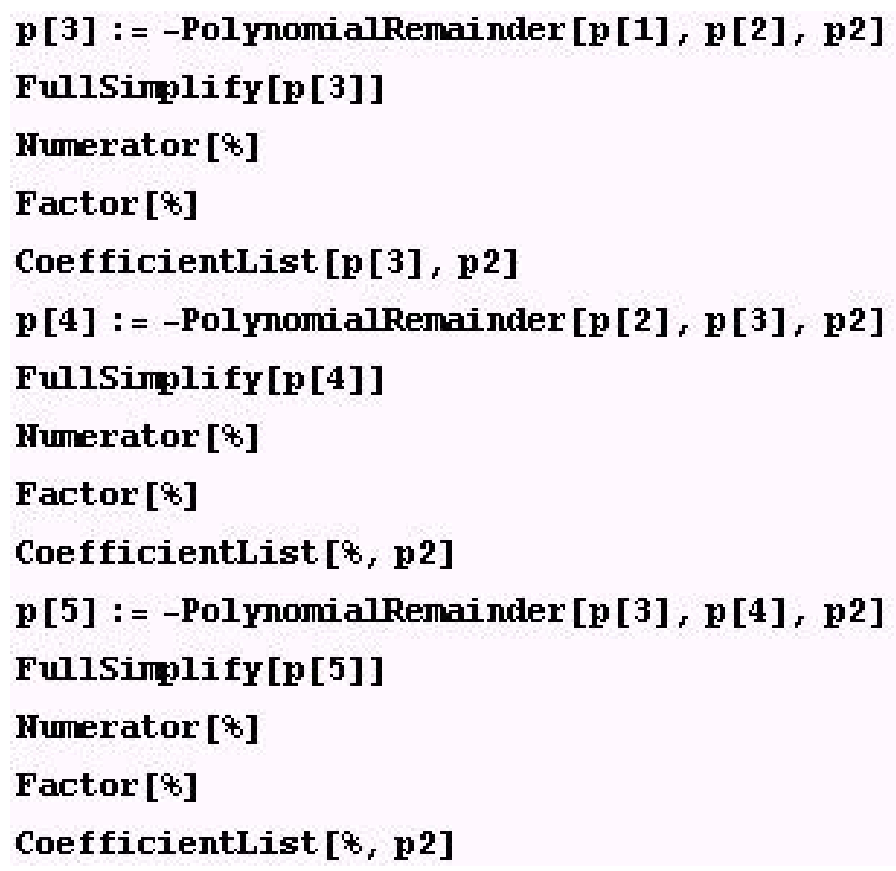

Nuestra ecuación (B.1) es una ecuación en $p_{2}$, recordemos que denota a $p_{\theta}$, donde $p_{2}$ varía en el intervalo $(-\infty,+\infty)$. Como algunos valores dependen de $h$ y ésta se encuentra en el intervalo $(-\infty,+\infty)$ veamos qué ocurre en los intervalos donde varía. Realizaremos un estudio numérico al respecto calculando los puntos críticos para valores concretos.

1. Caso en que en que hay dos puntos críticos, $h=h_{1}$ y $h=h_{2}$.

Tomamos valores de $\alpha$ y $\beta$ que verifiquen $27 \alpha \beta^{4}-128>0$.

Por ejemplo $\alpha=1, \beta=2$.

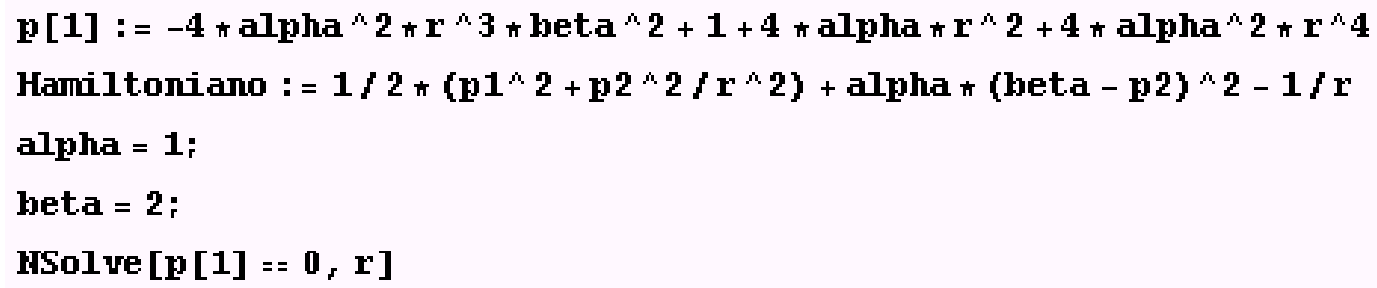

Si evaluamos $p_{2}$ y $\mathcal{H}$ en los valores obtenidos, se tienen los siguientes valores críticos del hamiltoniano

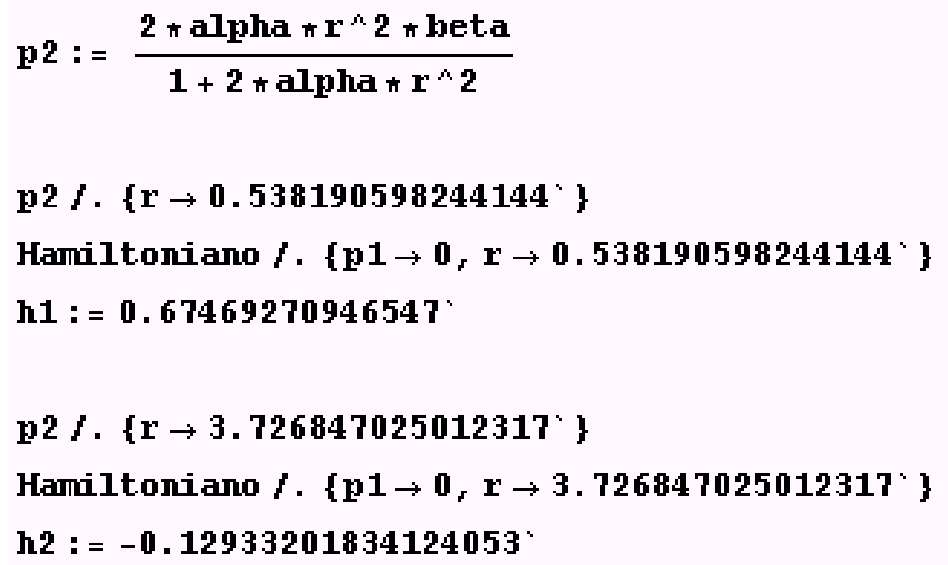


2. Caso en que hay un punto crítico doble, $h=h_{3}$.

Tomamos valores de $\alpha$ y $\beta$ que verifiquen $27 \alpha \beta^{4}-128=0$.

Por ejemplo $\alpha=\frac{128}{27}, \beta=1$.

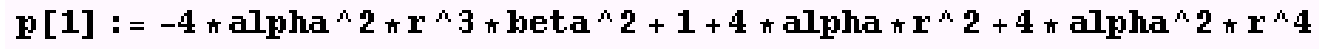

Hamiltoniano $:=1 / 2 \star\left(\mathrm{p}^{\wedge} 2+\mathrm{p}^{\wedge} 2 / \mathrm{r}^{\wedge} 2\right)+$ alpha * (beta $\left.-\mathrm{p} 2\right)^{\wedge} 2-1 / \mathrm{r}$

alpha $=128 / 27$

beta $=1$

HSolve $[p[1]==0, \mathbf{r}]$

Si evaluamos $p_{2}$ y $\mathcal{H}$ en el valor obtenido, se tiene el siguiente valor crítico del hamiltoniano

$$
\begin{aligned}
& \text { p2 }:=\frac{2 \star \text { alpha } \star r^{\wedge} 2 \star \text { beta }}{1+2 \star \text { alpha } * r^{\wedge} 2} \\
& \text { p2 } / .\left\{r \rightarrow 0.5625^{`}\right\} \\
& \text { Hamiltoniano } / .\left\{p 1 \rightarrow 0, r \rightarrow 0.5625^{`}\right\} \\
& \text { h3 }:=-0.5925925925925926^{\prime}
\end{aligned}
$$

3. Caso en que no hay puntos críticos.

Tomamos valores de $\alpha$ y $\beta$ que verifiquen $27 \alpha \beta^{4}-128<0$.

Por ejemplo $\alpha=1, \beta=1$.

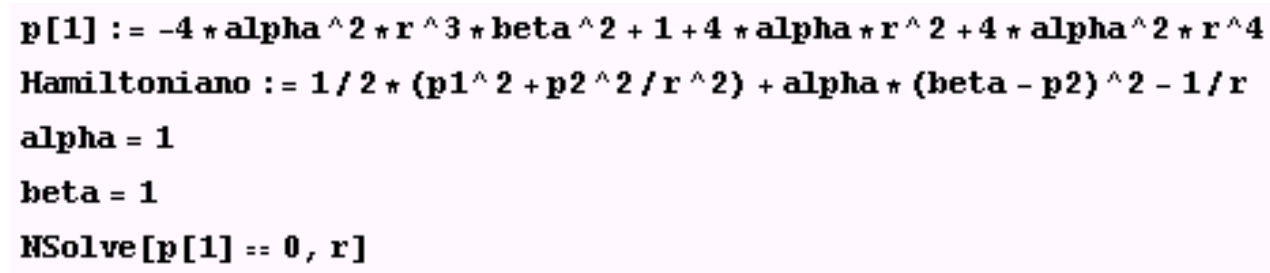

Por tanto, hacemos para cada caso

1.a) Primer punto crítico $h=h_{1}$.

$$
\begin{aligned}
& \text { alpha }=1 \\
& \text { beta }=2 \\
& r=0.538190598244144 \\
& \text { p2 }=0.7336147478371357 \\
& h=0.6746927094654747
\end{aligned}
$$

Veamos los cambios de signo $V(-\infty)$.

Obsérvese antes que se tienen fijados los signos $V(+,-$, , , ), por lo que definimos 


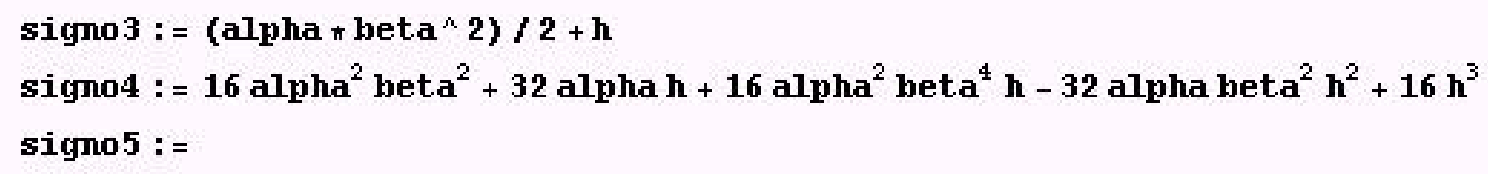

(alpha beta $\left.\mathrm{a}^{2}+2 \mathrm{~h}\right)^{2}$ (-8 alpha ${ }^{2}+$ alpha $^{3}$ beta $a^{4}-20$ alpha $^{2}$ beta $a^{2} h+2$ alpha $^{3}$ beta $a^{6} h-8$ alpha $h^{2}-6$ alpha ${ }^{2}$ beta ${ }^{4} h^{2}+$ 6 alpha beta ${ }^{2} h^{3}-2 h^{4}$ )

y evaluamos en los valores de $\alpha, \beta, \mathrm{y} h$ que hemos obtenido

signo3 $/$. $\{$ alpha $\rightarrow 1$, beta $\rightarrow 2$, h $\rightarrow 0.6746927094654747\}$
signo4 $/$ alpha $\rightarrow 1$, beta $\rightarrow 2$, h $\rightarrow 0.6746927094654747\}$
signo5 $/$ (alpha $\rightarrow 1$, beta $\rightarrow 2$, h $\rightarrow 0.6746927094654747\}$

Así pues, tenemos $V(+,-,+,-,+)$, es decir, cuatro cambios de signo.

Veamos los cambios de signo de $V(+\infty)$.

Tenemos $V(+,+,+,+,+)$, cero cambios de signo.

Por tanto

$$
V(-\infty)-V(+\infty)=4-0=4
$$

es decir, tenemos cuatro raíces reales.

1.b) Segundo punto crítico $h=h_{2}$.

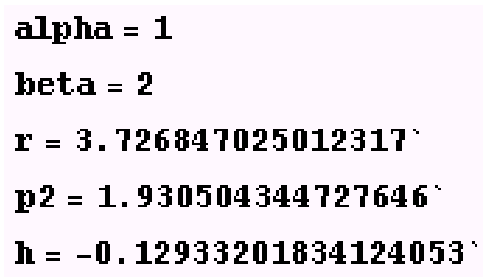

Veamos los cambios de signo de $V(-\infty)$.

$$
\begin{aligned}
& \text { signo } 3 / \text {. \{alpha } \rightarrow 1 \text {, beta } \rightarrow 2, \mathrm{~h} \rightarrow-0.12933201834124053 \text { \} } \\
& \text { signo4 } / \text {. \{alpha } \rightarrow 1 \text {, beta } \rightarrow 2, h \rightarrow-0.12933201834124053^{\prime} \text { \} } \\
& \text { signo5 } / \text {. \{alpha } \rightarrow 1 \text {, beta } \rightarrow 2, h \rightarrow-0.12933201834124053 \text { \} }
\end{aligned}
$$

Por tanto queda $V(+,-,+,-,+)$, es decir cuatro cambios de signo.

Viendo los cambios de signo de $V(+\infty)$, tenemos $V(+,+,+,+,+)$, es decir cero cambios de signo

Por tanto

$$
V(-\infty)-V(+\infty)=4-0=4
$$

lo que significa que tenemos cuatro raíces reales. 
1.c) Casos intermedios.

Vamos a estudiar los cambios de signo en los casos intermedios, tomando para ello como valores de $h$ los siguientes:

$$
\begin{aligned}
& h=-1 \text { para } h<h_{2} \\
& h=-0,01 \text { para } h_{2}<h<0 \\
& h=0,4 \text { para } 0<h<h_{1} \\
& h=1 \text { para } h_{1}<h
\end{aligned}
$$

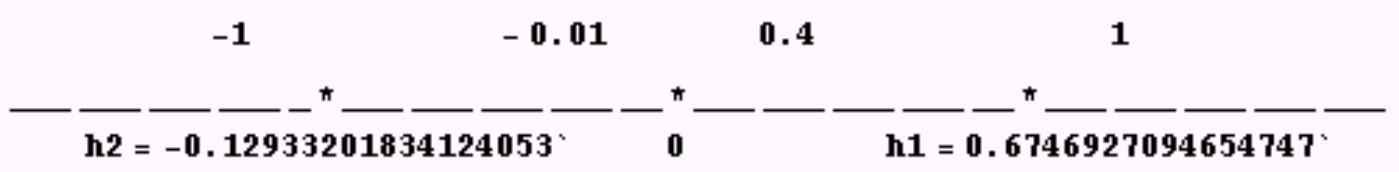

- Sea $h=-1$.

$$
\begin{aligned}
& \text { signo3 } / \text { \{alpha } \rightarrow 1 \text {, beta } \rightarrow 2, h \rightarrow-1\} \\
& \text { signo4 } / .\{\text { alpha } \rightarrow 1 \text {, beta } \rightarrow 2, h \rightarrow-1\} \\
& \text { signo5 } / .\{\text { alpha } \rightarrow 1 \text {, beta } \rightarrow 2, h \rightarrow-1\}
\end{aligned}
$$

Así pues, obtenemos para $V(-\infty): V(+,-,+,+,-)$, que son tres cambios de signo.

Para $V(+\infty): V(+,+,+,-,-)$, que nos da un solo cambio de signo.

Por tanto

$$
V(-\infty)-V(+\infty)=3-1=2
$$

es decir, tenemos dos raíces reales.

- Caso en que $h=-0,01$.

$$
\begin{aligned}
& \text { signo3 } / .\{\text { alpha } \rightarrow 1, \text { beta } \rightarrow 2, h \rightarrow-0.01\} \\
& \text { signo4 } / .\{\text { alpha } \rightarrow 1, \text { beta } \rightarrow 2, h \rightarrow-0.01\} \\
& \text { signo5 } / .\{\text { alpha } \rightarrow 1, \text { beta } \rightarrow 2, h \rightarrow-0.01\}
\end{aligned}
$$

Es decir, que para $V(-\infty)$ tenemos: $V(+,-,+,-,+)$, que son cuatro cambios de signo.

Veamos los cambios de signo $V(+\infty)$.

Tenemos $V(+,+,+,+,+)$, que son cero cambios de signo.

Por tanto

$$
V(-\infty)-V(+\infty)=4-0=4
$$

lo que nos da cuatro raíces reales.

- Tomando $h=0,4$

$$
\begin{aligned}
& \text { signo } 3 / .\{\text { alpha } \rightarrow 1, \text { beta } \rightarrow 2, h \rightarrow 0.4\} \\
& \text { signo4 } / .\{\text { alpha } \rightarrow 1, \text { beta } \rightarrow 2, h \rightarrow 0.4\} \\
& \text { signo5 } / .\{\text { alpha } \rightarrow 1, \text { beta } \rightarrow 2, h \rightarrow 0.4\}
\end{aligned}
$$


Es decir, que para $V(-\infty)$ tenemos $V(+,-,+,-,+)$, que son cuatro cambios de signo.

Veamos los cambios de signo $V(+\infty)$.

Tenemos $V(+,+,+,+,+)$, que son cero cambios de signo.

Por tanto

$$
V(-\infty)-V(+\infty)=4-0=4
$$

es decir, tenemos cuatro raíces reales.

- Sea $h=1$.

$$
\begin{aligned}
& \text { signo3 } / \text { \{alpha } \rightarrow 1 \text {, beta } \rightarrow 2, h \rightarrow 1\} \\
& \text { signo4 } / .\{\text { alpha } \rightarrow 1 \text {, beta } \rightarrow 2, h \rightarrow 1\} \\
& \text { signo5 } / .\{\text { alpha } \rightarrow 1 \text {, beta } \rightarrow 2, h \rightarrow 1\}
\end{aligned}
$$

Es decir, que para $V(-\infty)$ tenemos $V(+,-,+,-,-)$, y esto son tres cambios de signo

Veamos los cambios de signo $V(+\infty)$.

Tenemos $V(+,+,+,-,-)$, que nos da un solo cambio de signo.

Por tanto

$$
V(-\infty)-V(+\infty)=3-1=2
$$

lo que significa que tenemos dos raíces reales.

2.a) Un punto crítico doble, $h=h_{3}$

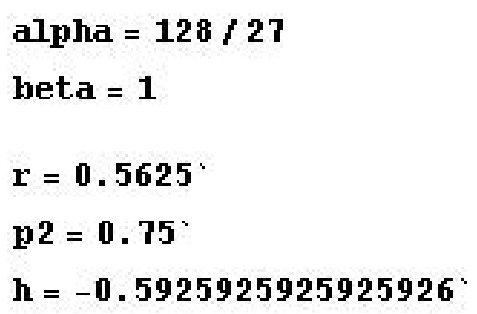

Veamos los cambios de signo de $V(-\infty)$ y $V(+\infty)$

$$
\begin{aligned}
& \text { signo3 /. \{alpha } \rightarrow 128 / 27 \text {, beta } \rightarrow 1, \text { h } \rightarrow-0.5925925925925926 \text { ' } \\
& \text { signo4 } / \text {. \{alpha } \rightarrow 128 / 27 \text {, beta } \rightarrow 1, \mathrm{~h} \rightarrow-0.5925925925925926 \text { \} } \\
& \text { signo5 /. \{alpha } \rightarrow 128 / 27 \text {, beta } \rightarrow 1, \text { h } \rightarrow-0.5925925925925926 \text { \} }
\end{aligned}
$$

Tenemos para $V(-\infty): V(+,-,+,+,-)$, que son tres cambios de signo.

Veamos los cambios de signo $V(+\infty)$.

Tenemos $V(+,+,+,-,-)$, que nos da un solo cambio de signo.

Por tanto

$$
V(-\infty)-V(+\infty)=3-1=2
$$

lo que nos da dos raíces reales.

2.b) Casos intermedios

Vamos a estudiar los cambios de signo en los casos intermedios, tomando como valores de $h$ los siguientes: 


$$
\begin{aligned}
& h=-1 \text { para } h<h_{3} \\
& h=-0,2 \text { para } h_{3}<h<0 \\
& h=1 \text { para } 0 \leq h
\end{aligned}
$$

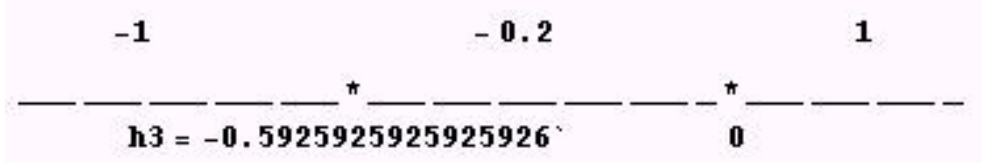

- Sea $h=-1$.

signo3 $/$. \{alpha $\rightarrow 128 / 27$, beta $\rightarrow 1, h \rightarrow-1$ \}

signo4 $/$. \{alpha $\rightarrow 128 / 27$, beta $\rightarrow 1, h \rightarrow-1$ \}

signo5 $/$. \{alpha $\rightarrow 128 / 27$, beta $\rightarrow 1, h \rightarrow-1\}$

Es decir, tenemos para $V(-\infty): V(+,-,+,+,-)$, que son tres cambios de signo.

Veamos los cambios de signo $V(+\infty)$.

Tenemos $V(+,+,+,-,-)$, que es un solo cambio de signo.

Por tanto

$$
V(-\infty)-V(+\infty)=3-1=2
$$

lo que quiere decir que tenemos dos raíces reales.

- Tomando ahora $h=-0,2$.

$$
\begin{aligned}
& \text { signo3 } / .\{\text { alpha } \rightarrow 128 / 27, \text { beta } \rightarrow 1, h \rightarrow-0.2\} \\
& \text { signo4 } / .\{\text { alpha } \rightarrow 128 / 27, \text { beta } \rightarrow 1, h \rightarrow-0.2\} \\
& \text { signo5 } / .\{\text { alpha } \rightarrow 128 / 27, \text { beta } \rightarrow 1, h \rightarrow-0.2\}
\end{aligned}
$$

Es decir, que para $V(-\infty)$ tenemos $V(+,-,+,-,-)$, que son tres cambios de signo.

Veamos los cambios de signo $V(+\infty)$.

Tenemos $V(+,+,+,+,-)$, que es un solo cambio de signo.

Por tanto

$$
V(-\infty)-V(+\infty)=3-1=2
$$

es decir, tenemos dos raíces reales.

- Caso en que $h=1$.

$$
\begin{aligned}
& \text { signo3 } / .\{\text { alpha } \rightarrow 128 / 27, \text { beta } \rightarrow 1, h \rightarrow 1\} \\
& \text { signo4 } / .\{\text { alpha } \rightarrow 128 / 27, \text { beta } \rightarrow 1, h \rightarrow 1\} \\
& \text { signo5 } / .\{\text { alpha } \rightarrow 128 / 27, \text { beta } \rightarrow 1, h \rightarrow 1\}
\end{aligned}
$$

Es decir, que para $V(-\infty)$ tenemos $V(+,-,+,-,-)$, que son tres cambios de signo.

Veamos los cambios de signo $V(+\infty)$.

Tenemos $V(+,+,+,+,-)$, que es un solo cambio de signo. 
Por tanto

$$
V(-\infty)-V(+\infty)=3-1=2
$$

es decir, tenemos dos raíces reales.

3.a) Casos intermedios.

Tomamos como valores de $h$ :

$h=-0,129332$ para $h<0$

$h=1$ para $0 \leq h$

$-0.129332$

1

- Tomamos ahora $h=-0,129332$

signo3 $/$. \{alpha $->1$, beta $\rightarrow 1, h \rightarrow-0.129332\}$

signo4 $/$. \{alpha $\rightarrow 1$, beta $\rightarrow 1, h \rightarrow-0.129332\}$

signo5 $/$. \{alpha $\rightarrow 1$, beta $\rightarrow 1, h \rightarrow-0.129332\}$

Se tiene que para $V(-\infty)$ tenemos $V(+,-,+,-,-)$, que son tres cambios de signo.

Veamos los cambios de signo $V(+\infty)$.

Se obtiene $V(+,+,+,+,-)$, un solo cambio de signo.

Por tanto

$$
V(-\infty)-V(+\infty)=3-1=2
$$

es decir, tenemos dos raíces reales.

- Tomamos ahora $h=1$.

$$
\begin{aligned}
& \text { signo3 } / .\{\text { alpha } \rightarrow 1 \text {, beta } \rightarrow 1, h \rightarrow 1\} \\
& \text { signo4 } / .\{\text { alpha } \rightarrow 1 \text {, beta } \rightarrow 1, h \rightarrow 1\} \\
& \text { signo5 } / .\{\text { alpha } \rightarrow 1, \text { beta } \rightarrow 1, h \rightarrow 1\}
\end{aligned}
$$

Se tiene que para $V(-\infty)$ tenemos $V(+,-,+,-,-)$, que son tres cambios de signo.

Veamos los cambios de signo $V(+\infty)$.

Se obtiene $V(+,+,+,+,-)$, un cambio de signo.

Por tanto

$$
V(-\infty)-V(+\infty)=3-1=2
$$

lo que nos da dos raíces reales. 


\section{En resumen:}

\begin{tabular}{|c|}
\hline Caso 1: $27 \alpha \beta^{4}-128>0$ \\
\hline Para $h=h_{1}$, cuatro raíces reales \\
\hline Para $h=h_{2}$, cuatro raíces reales \\
\hline Para $h<h_{2}: h=-1$, dos raíces reales \\
\hline Para $h_{2}<h<0: h=-0,01$, cuatro raíces reales \\
\hline Para $0<h<h_{1}: h=0,4$, cuatro raíces reales \\
\hline Para $h_{1}<h: h=1$, dos raíces reales \\
\hline
\end{tabular}

Tabla B.1: Número de raíces del polinomio (B.1) para el caso en que $27 \alpha \beta^{4}-128>0$.

\begin{tabular}{|c|}
\hline Caso $2: 27 \alpha \beta^{4}-128=0$ \\
\hline Para $h=h_{3}$, dos raíces reales \\
\hline Para $h<h_{3}: h=-1$, dos raíces reales \\
\hline Para $h_{3}<h<0: h=-0,2$, dos raíces reales \\
\hline Para $0 \leq h: h=1$, dos raíces reales \\
\hline
\end{tabular}

Tabla B.2: Número de raíces del polinomio (B.1) para el caso en que $27 \alpha \beta^{4}-128=0$.

\begin{tabular}{|c|}
\hline Caso $3: 27 \alpha \beta^{4}-128<0$ \\
\hline Para $h=-0,129332$, dos raíces reales \\
\hline Para $h=1$, dos raíces reales \\
\hline
\end{tabular}

Tabla B.3: Número de raíces del polinomio (B.1) para el caso en que $27 \alpha \beta^{4}-128<0$. 



\section{Apéndice $\mathrm{C}$}

\section{Topología de $I_{h k}$}

En esta sección se va a hacer el estudio de las raíces del polinomio

$$
p=-k^{2}+2 r+\left(-2 \alpha \beta^{2}+2 h+4 \alpha \beta k-2 \alpha k^{2}\right) r^{2}
$$

donde $k=p_{\theta}$.

Este polinomio se obtiene de despejar $p_{r}^{2}$ de la ecuación

$$
-h+\frac{1}{2}\left(p_{r}^{2}+\frac{p_{\theta}^{2}}{r^{2}}\right)+\alpha\left(\beta-p_{\theta}\right)^{2}-\frac{1}{r}=0
$$

y sustituir $p_{\theta}$ por $k$.

El código utilizado es el siguiente:

$$
f:=-h+\text { alpha }(\text { beta }-p 2)^{2}+\frac{1}{2}\left(p 1^{2}+\frac{p 2^{2}}{r^{2}}\right)-\frac{1}{r}
$$

\section{Expand [f]}

f $/ .\left\{\mathbf{p 1} \mathbf{1}^{\wedge} \rightarrow \mathbf{Y}\right\}$

Solve $\left.[-\mathrm{h}+\text { alpha (beta }-\mathrm{p} 2)^{2}-\frac{1}{\mathrm{r}}+\frac{1}{2}\left(\frac{\mathrm{p} 2^{2}}{\mathrm{r}^{2}}+\mathrm{Y}\right)=0, \mathrm{Y}\right]$

$Y:=\frac{1}{r^{2}}\left(-p 2^{2}+2 r-2\right.$ alpha beta $a^{2} r^{2}+2 h r^{2}+4$ alpha beta $p 2 r^{2}-2$ alpha $\left.p 2^{2} r^{2}\right)$

Humerator [Y]

Polinomio $:=-p 2^{2}+2 r-2$ alpha beta $a^{2} r^{2}+2 h r^{2}+4$ alpha beta $p 2 r^{2}-2$ alpha $p 2^{2} r^{2}$

Polinomio $/ .\{\mathrm{p} 2 \rightarrow \mathrm{k}\}$

Vamos a calcular la sucesión de Sturm para este polinomio, al que llamaremos $p[1]$. 


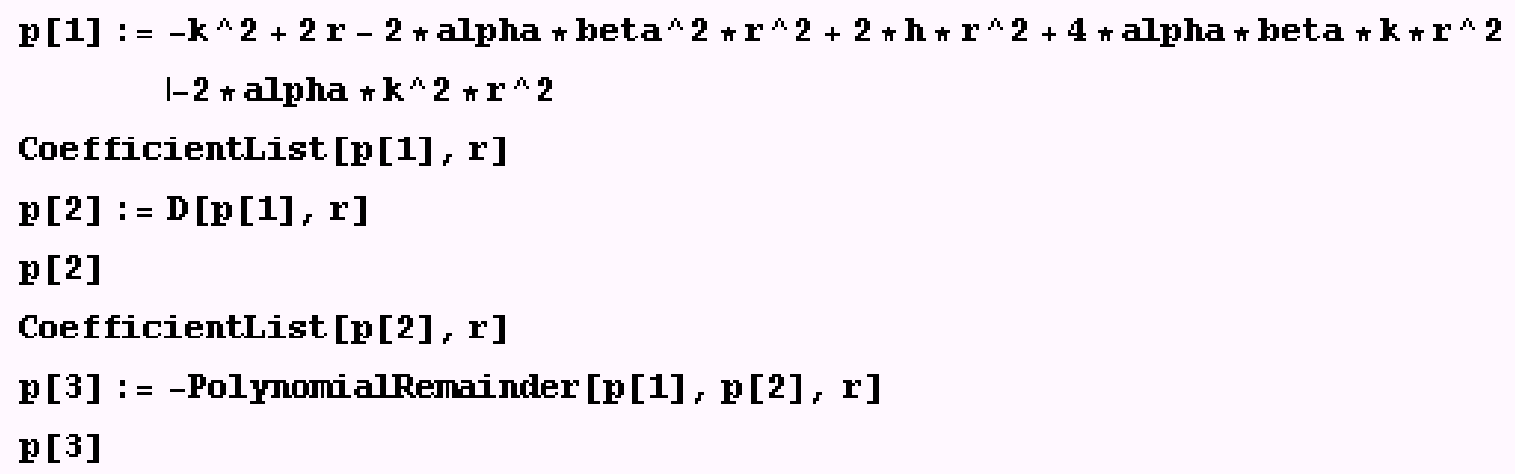

En este caso, como $r \in \mathbb{R}^{+}$tendremos que estudiar el polinomio de Sturm para $V(0)$ y $V(+\infty)$, que son

$$
\begin{gathered}
V(0)=\left(-k^{2}, 2,-\frac{1}{2\left(-h+\alpha(\beta-k)^{2}\right)}+k^{2}\right) \\
V(+\infty)=\left(2\left(h-\alpha(\beta-k)^{2}\right), 4\left(h-\alpha(\beta-k)^{2}\right),-\frac{1}{2\left(-h+\alpha(\beta-k)^{2}\right)}+k^{2}\right)
\end{gathered}
$$

Observación 74 Obsérvese que en $V(+\infty)$ el segundo coeficiente es el doble del primero y que en $V(0)$ tenemos siempre que $V(0)=(-,+, \quad)$, por lo que para ver el número de raíces bastará estudiar el signo de

Finalmente, en cada uno de los casos obtenidos, estudiaremos cómo son las curvas.

- $h-\alpha(\beta-k)^{2}$

$-\frac{1}{2\left(-h+\alpha(\beta-k)^{2}\right)}+k^{2}$

1. CASO 1 : Dos puntos críticos.

a) Para $h=-1$

alpha $=1$

beta $=2$

$\mathbf{h}=\mathbf{- 1}$

$\ll$ Algebra 'InequalitySolve

InequalitySolve $\left[-\frac{1}{2\left(-h+\text { alpha }(\text { beta }-k)^{2}\right)}+k^{2}<0, k\right]$

HSolve $\left[-1+10 \# 1^{2}-8 \# 1^{3}+2 \# 1^{4}\right]$ 
Por tanto

- Si $c_{2}=-0,28363796061324487^{`}<k<0$

$$
\begin{aligned}
& V(0)=(-,+,-) \\
& V(+\infty)=(-,-,-)
\end{aligned}
$$

Luego

$$
V(0)-V(+\infty)=2
$$

Es decir, todas las curvas son acotadas.

- Para $k=0$ es no acotada.

- Para $0<k<0,3697213408365262^{6}=c_{1}$

$$
\begin{aligned}
& V(0)=(-,+,-) \\
& V(+\infty)=(-,-,-)
\end{aligned}
$$

Luego

$$
V(0)-V(+\infty)=2
$$

por lo que todas las curvas son acotadas.

b) Para $h=h_{2}=-0,12933201834124053$

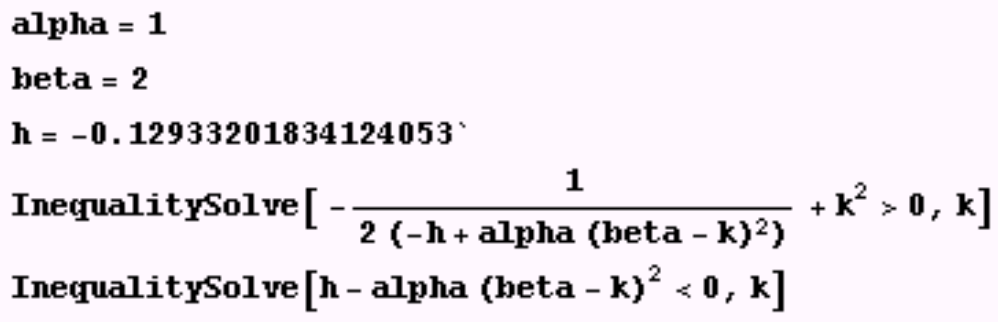

Por tanto

- Si $c_{2}=-0,30332^{\natural}<k<0$

$$
\begin{aligned}
& V(0)=(-,+,-) \\
& V(+\infty)=(-,-,-)
\end{aligned}
$$

Luego

$$
V(0)-V(+\infty)=2
$$

Es decir, todas las curvas son acotadas.

- Para $k=0$ es no acotada. 
- Para $0<k<0,442311=c_{1}$

$V(0)=(-,+,-)$
$V(+\infty)=(-,-,-)$

Luego

$$
V(0)-V(+\infty)=2
$$

por lo que obtenemos que todas las curvas son acotadas.

c) Para $h=-0,01$

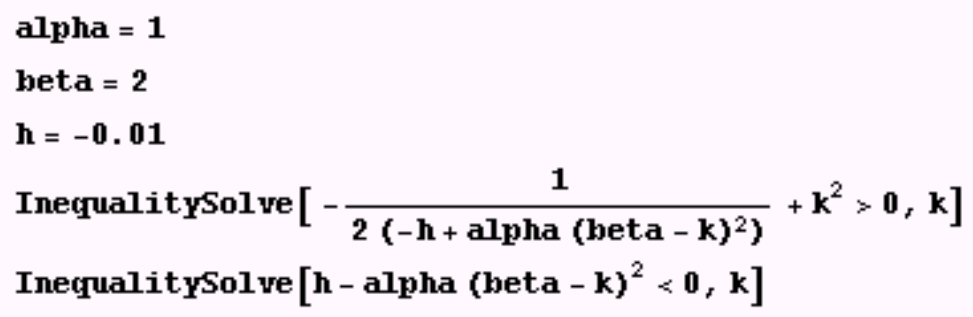

Por tanto

- Para $c_{2}=-0,3065629648763765^{`}<k<0$

$$
\begin{aligned}
& V(0)=(-,+,-) \\
& V(+\infty)=(-,-,-)
\end{aligned}
$$

Luego

$$
V(0)-V(+\infty)=2
$$

Así pues, todas las curvas son acotadas.

- Para $k=0$ es no acotada.

- Para $0<k<0,45880389985380304^{`}=c_{1}$

$$
\begin{aligned}
& V(0)=(-,+,-) \\
& V(+\infty)=(-,-,-)
\end{aligned}
$$

Luego

$$
V(0)-V(+\infty)=2
$$

Es decir, todas las curvas son acotadas.

- Para $c_{3}=1,556999290660659^{`}<k<2,291872432587733^{`}=c_{1}$

$$
V(0)=(-,+,-)
$$


$V(+\infty)=(-,-,-)$

Luego

$$
V(0)-V(+\infty)=2
$$

por lo que todas las curvas son acotadas.

d) Para $h=0,4$

$$
\begin{aligned}
& \text { alpha }=1 \\
& \text { beta }=2 \\
& h=0.4
\end{aligned}
$$

InequalitySolve $\left[-\frac{1}{2\left(-h+\text { alpha }(\text { beta }-k)^{2}\right)}+k^{2}<0, k\right]$

InequalitySolve [h - alpha (beta $\left.-k)^{2}<0, k\right]$

HSolve [h - alpha (beta $\left.-k)^{2}\right]$

- Para $c_{2}=-0,31719946142257877^{`}<k<0$

$$
\begin{aligned}
& V(0)=(-,+,-) \\
& V(+\infty)=(-,-,-)
\end{aligned}
$$

Luego

$$
V(0)-V(+\infty)=2
$$

que nos dice que todas las curvas son acotadas.

- Para $k=0$ es no acotada.

- Para $0<k<0,5351813767342671^{6}=c_{1}$

$$
\begin{aligned}
& V(0)=(-,+,-) \\
& V(+\infty)=(-,-,-)
\end{aligned}
$$

Luego

$$
V(0)-V(+\infty)=2
$$

Es decir, todas las curvas son acotadas.

- Para $c_{3}=1,0969262383446852^{`}<k<1,3675444679663242^{`}=a_{1}$

$$
\begin{aligned}
& V(0)=(-,+,-) \\
& V(+\infty)=(-,-,-)
\end{aligned}
$$


Luego

$$
V(0)-V(+\infty)=2
$$

por lo que todas las curvas son acotadas.

- Para $a_{1}=1,3675444679663242^{`}<k<2,632455532033952^{`}=a_{2}$

$$
\begin{aligned}
& V(0)=(-,+,+) \\
& V(+\infty)=(+,+,+)
\end{aligned}
$$

Luego

$$
V(0)-V(+\infty)=1
$$

que nos dice que las curvas son abiertasno acotadas.

- Para $a_{2}=2,632455532033952^{6}<k<2,6850918463433535^{6}=c_{4}$

$$
\begin{aligned}
& V(0)=(-,+,-) \\
& V(+\infty)=(-,-,-)
\end{aligned}
$$

Luego

$$
V(0)-V(+\infty)=2
$$

Es decir, todas las curvas son acotadas.

e) Para $h=h_{1}=0,6746927094654747^{\text {‘ }}$

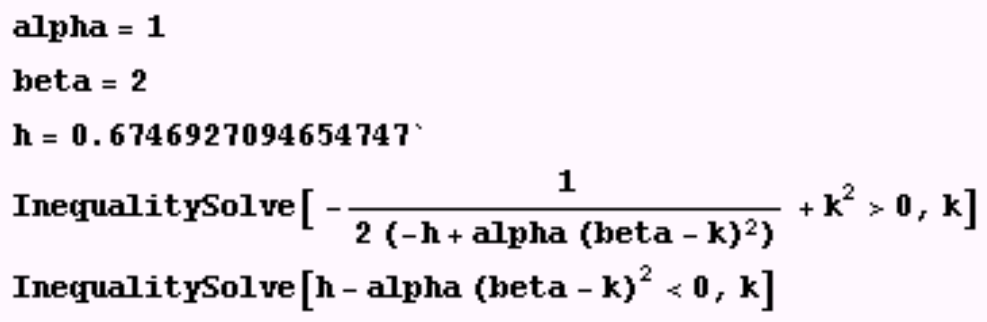

Por tanto

- Para $c_{2}=-0,325082782177043^{6}<k<0$

$$
\begin{aligned}
& V(0)=(-,+,-) \\
& V(+\infty)=(-,-,-)
\end{aligned}
$$

Luego

$$
V(0)-V(+\infty)=2
$$

Así pues, todas las curvas son acotadas.

- Para $k=0$ no acotada. 
- Para $0<k<1,1786031960949235^{6}=c_{1}$

$$
\begin{aligned}
& V(0)=(-,+,-) \\
& V(+\infty)=(-,-,-)
\end{aligned}
$$

Luego

$$
V(0)-V(+\infty)=2
$$

lo que significa que todas las curvas son acotadas.

- Para $a_{1}=1,1786031960949235^{`}<k<2,8213968039050767^{`}=a_{2}$

$$
\begin{aligned}
& V(0)=(-,+,+) \\
& V(+\infty)=(+,+,+)
\end{aligned}
$$

Luego

$$
V(0)-V(+\infty)=1
$$

Es decir, las curvas son no acotadas.

- Para $a_{2}=2,8213968039050767^{`}<k<2,857853286502772^{`}=c_{1}$

$$
\begin{aligned}
& V(0)=(-,+,-) \\
& V(+\infty)=(-,-,-)
\end{aligned}
$$

Luego

$$
V(0)-V(+\infty)=2
$$

lo que nos da que todas las curvas son acotadas.

f) Para $h=1$

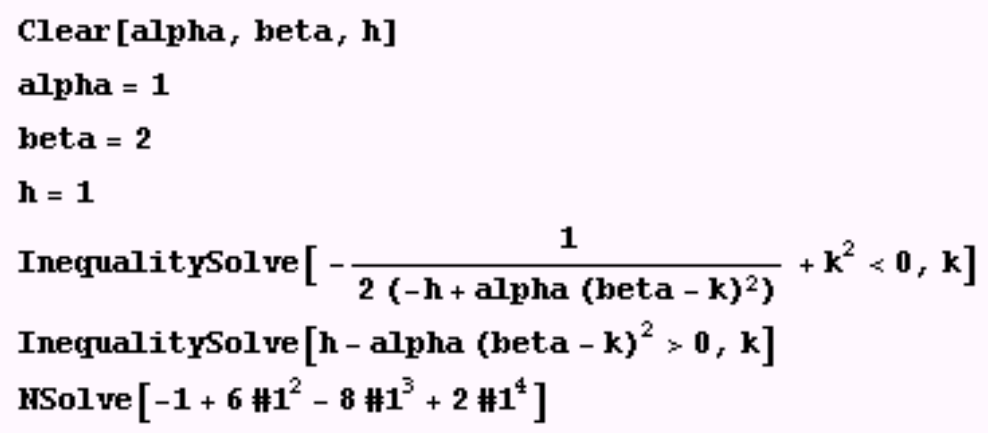

Por tanto

- Para $c_{2}=-0,3350995052996449^{`}<k<0$

$$
V(0)=(-,+,-)
$$


$V(+\infty)=(-,-,-)$

Luego

$$
V(0)-V(+\infty)=2
$$

lo que nos da que todas las curvas son acotadas.

- Para $k=0$ es no acotada.

- Para $0<k<1=a_{1}$

$$
\begin{aligned}
& V(0)=(-,+,-) \\
& V(+\infty)=(-,-,-)
\end{aligned}
$$

Luego

$$
V(0)-V(+\infty)=2
$$

Es decir, todas las curvas son acotadas.

- Para $a_{1}=1<k<3=a_{2}$

$$
\begin{aligned}
& V(0)=(-,+,+) \\
& V(+\infty)=(+,+,+)
\end{aligned}
$$

Luego

$$
V(0)-V(+\infty)=1
$$

Así pues, las curvas son no acotadas.

- Para $a_{2}=3<k<3,026923394158243^{\circ}=c_{1}$

$$
\begin{aligned}
& V(0)=(-,+,-) \\
& V(+\infty)=(-,-,-)
\end{aligned}
$$

Luego

$$
V(0)-V(+\infty)=2
$$

por lo que todas las curvas son acotadas.

\section{CASO 2: un punto crítico doble}

a) Para $h=-1$

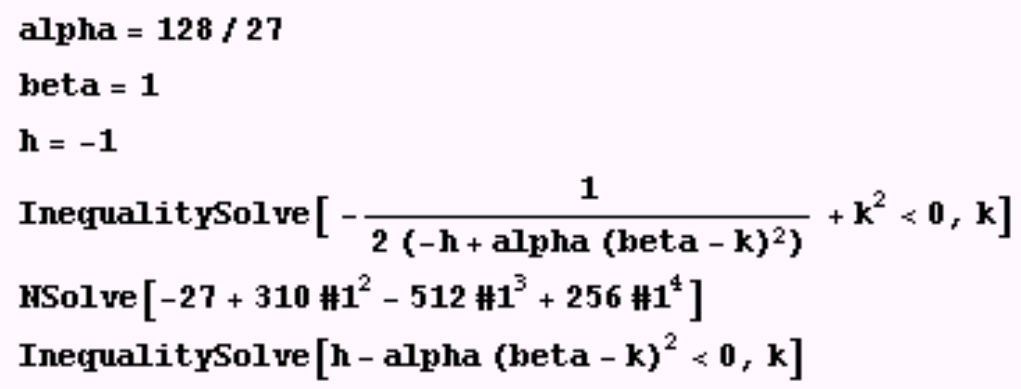


Por tanto

- Para $c_{2}=-0,24476965565666428^{\circ}<k<0$

$$
\begin{aligned}
& V(0)=(-,+,-) \\
& V(+\infty)=(-,-,-)
\end{aligned}
$$

Luego

$$
V(0)-V(+\infty)=2
$$

lo que nos da que todas las curvas son acotadas.

- Para $k=0$ es no acotada.

- Para $0<k<0,45630303815292816=c_{1}$

$$
\begin{aligned}
& V(0)=(-,+,-) \\
& V(+\infty)=(-,-,-)
\end{aligned}
$$

Luego

$$
V(0)-V(+\infty)=2
$$

Así pues, todas las curvas son acotadas.

b) Para $h=h_{3}=-0,5925925925925926^{\circ}$

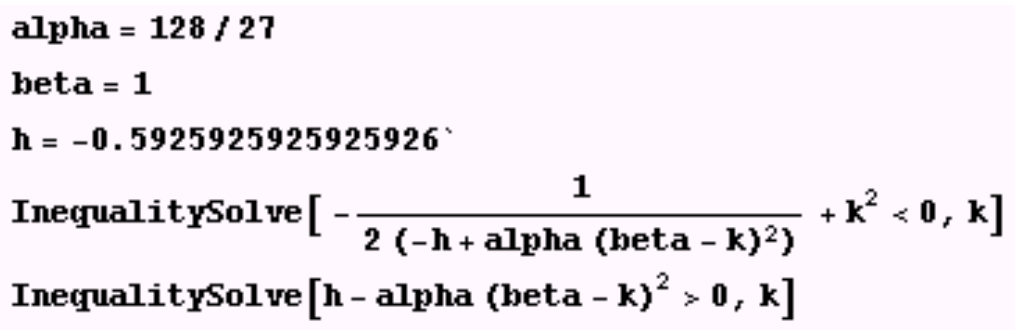

Por tanto

- Para $-0,25<k<0$

$$
\begin{aligned}
& V(0)=(-,+,-) \\
& V(+\infty)=(-,-,-)
\end{aligned}
$$

Luego

$$
V(0)-V(+\infty)=2
$$

Es decir, todas las curvas son acotadas.

- Para $k=0$ es no acotada. 
- Para $0<k<0,754131$

$$
\begin{aligned}
& V(0)=(-,+,-) \\
& V(+\infty)=(-,-,-)
\end{aligned}
$$

Luego

$$
V(0)-V(+\infty)=2
$$

lo que significa que todas las curvas son acotadas.

c) Para $h=-0,02$.

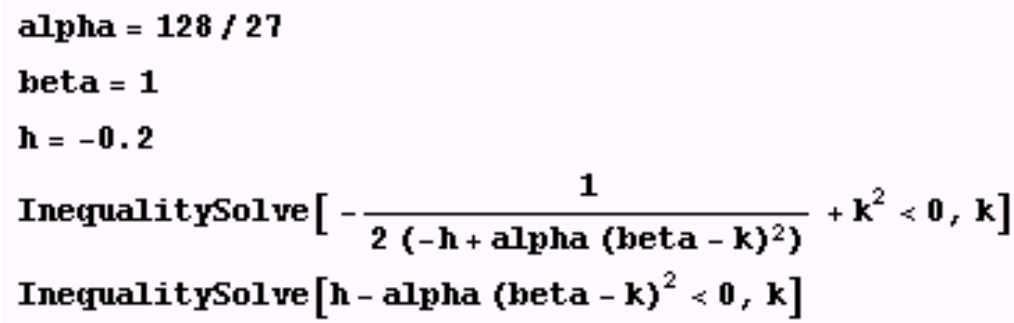

Por tanto

- Para $c_{2}=-0,25531297540579606^{6}<k<0$

$$
\begin{aligned}
& V(0)=(-,+,-) \\
& V(+\infty)=(-,-,-)
\end{aligned}
$$

Luego

$$
V(0)-V(+\infty)=2
$$

por lo que todas las curvas son acotadas.

- Para $k=0$ es no acotada.

- Para $0<k<1,182362573342762^{6}=c_{1}$

$$
\begin{aligned}
& V(0)=(-,+,-) \\
& V(+\infty)=(-,-,-)
\end{aligned}
$$

Luego

$$
V(0)-V(+\infty)=2
$$

Es decir, todas las curvas son acotadas. 
d) Para $h=1$.

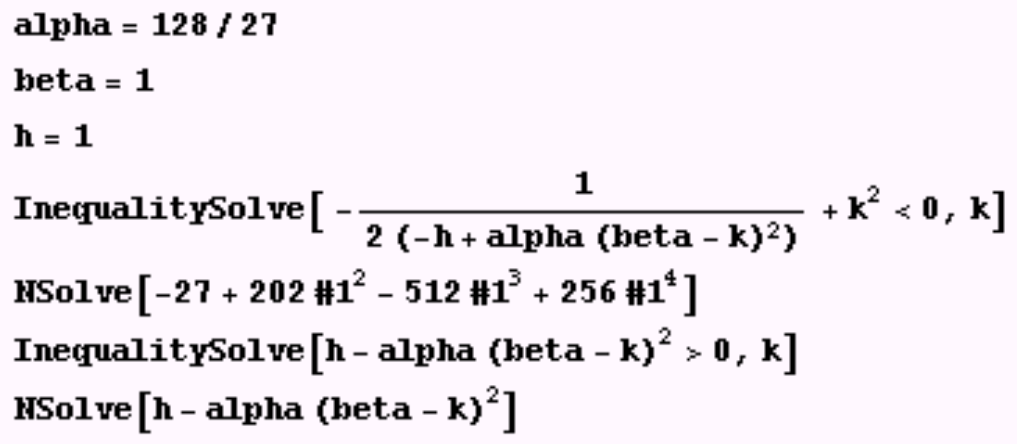

Por tanto

- Para $c_{2}=-0,273430246036642^{‘}<k<0$

$$
\begin{aligned}
& V(0)=(-,+,-) \\
& V(+\infty)=(-,-,-)
\end{aligned}
$$

Luego

$$
V(0)-V(+\infty)=2
$$

Así pues, todas las curvas son acotadas.

- Para $k=0$ es no acotada.

- Para $0<k<0,540720673228154^{6}=a_{1}$

$V(0)=(-,+,-)$

$V(+\infty)=(-,-,-)$

Luego

$$
V(0)-V(+\infty)=2
$$

lo que nos dice que todas las curvas son acotadas.

- Para $a_{1}=0,540720673228154^{6}<k<1,4592793267718456^{6}=a_{2}$

$$
\begin{aligned}
& V(0)=(-,+,+) \\
& V(+\infty)=(+,+,+)
\end{aligned}
$$

Luego

$$
V(0)-V(+\infty)=1
$$

que significa que las curvas son no acotadas. 
- Para $a_{2}=1,4592793267718456^{6}<k<1,5073058252492986^{6}=c_{1}$

$$
\begin{aligned}
& V(0)=(-,+,-) \\
& V(+\infty)=(-,-,-)
\end{aligned}
$$

Luego

$$
V(0)-V(+\infty)=2
$$

Es decir, todas las curvas son acotadas.

\section{CASO 3: ningún punto crítico.}

a) $h=-0,129332$.

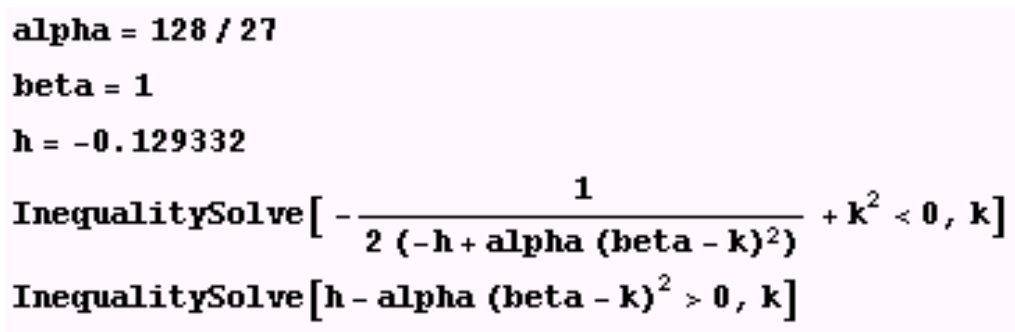

Por tanto

- Para $c_{2}=-0,256299<k<0$

$$
\begin{aligned}
& V(0)=(-,+,-) \\
& V(+\infty)=(-,-,-)
\end{aligned}
$$

Luego

$$
V(0)-V(+\infty)=2
$$

Así pues, todas las curvas son acotadas.

- Para $k=0$ es no acotada.

- Para $0<k<1,21121=c_{1}$

$$
\begin{aligned}
& V(0)=(-,+,-) \\
& V(+\infty)=(-,-,-)
\end{aligned}
$$

Luego

$$
V(0)-V(+\infty)=2
$$

lo que nos dice que todas las curvas son acotadas. 
b) $h=1$.

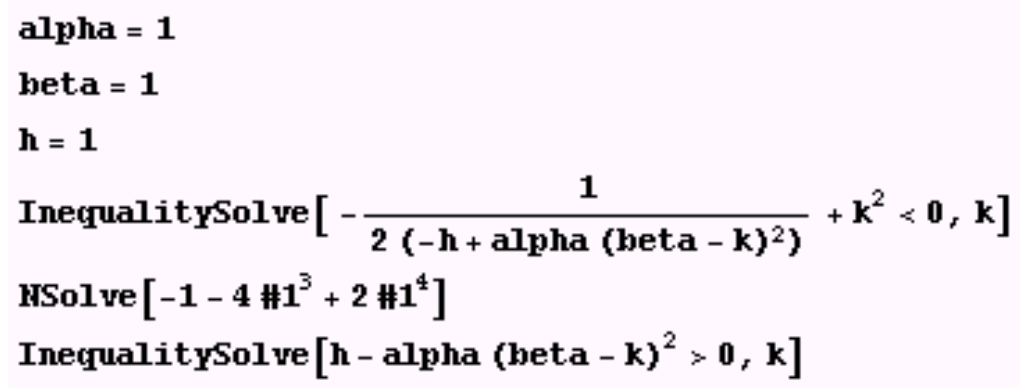

Por tanto

- Para $c_{2}=-0,5787862676985307^{`}<k<0$

$$
\begin{aligned}
& V(0)=(-,+,-) \\
& V(+\infty)=(-,-,-)
\end{aligned}
$$

Luego

$$
V(0)-V(+\infty)=2
$$

por lo que todas las curvas son acotadas.

- Para $k=0$ es no acotada.

- Para $a_{1}=0<k<2=a_{2}$

$$
\begin{aligned}
& V(0)=(-,+,-) \\
& V(+\infty)=(-,-,-)
\end{aligned}
$$

Luego

$$
V(0)-V(+\infty)=2
$$

Es decir, todas las curvas son acotadas.

- Para $a_{2}=2<k<2,0574124296879814^{6}=c_{1}$

$$
\begin{aligned}
& V(0)=(-,+,-) \\
& V(+\infty)=(-,-,-)
\end{aligned}
$$

Luego

$$
V(0)-V(+\infty)=2
$$

Así pues, todas las curvas son acotadas.

Observación 75 En todos los casos donde aparece el intervalo $\left(a_{1}, a_{2}\right)$ las curvas son no acotadas debido a que el polinomio p tiene una única raíz, que además es simple. Esto se puede comprobar al calcular las raíces del polinomio en $(-\infty,+\infty)$. En dicho casos nos salen dos raíces reales, una negativa y otra positiva. Es por ello que al hacer el algoritmo de Sturm en $(0,+\infty)$, obtenemos $V(0)-V(+\infty)=1$, que es una sola raíz. 


\section{En resumen:}

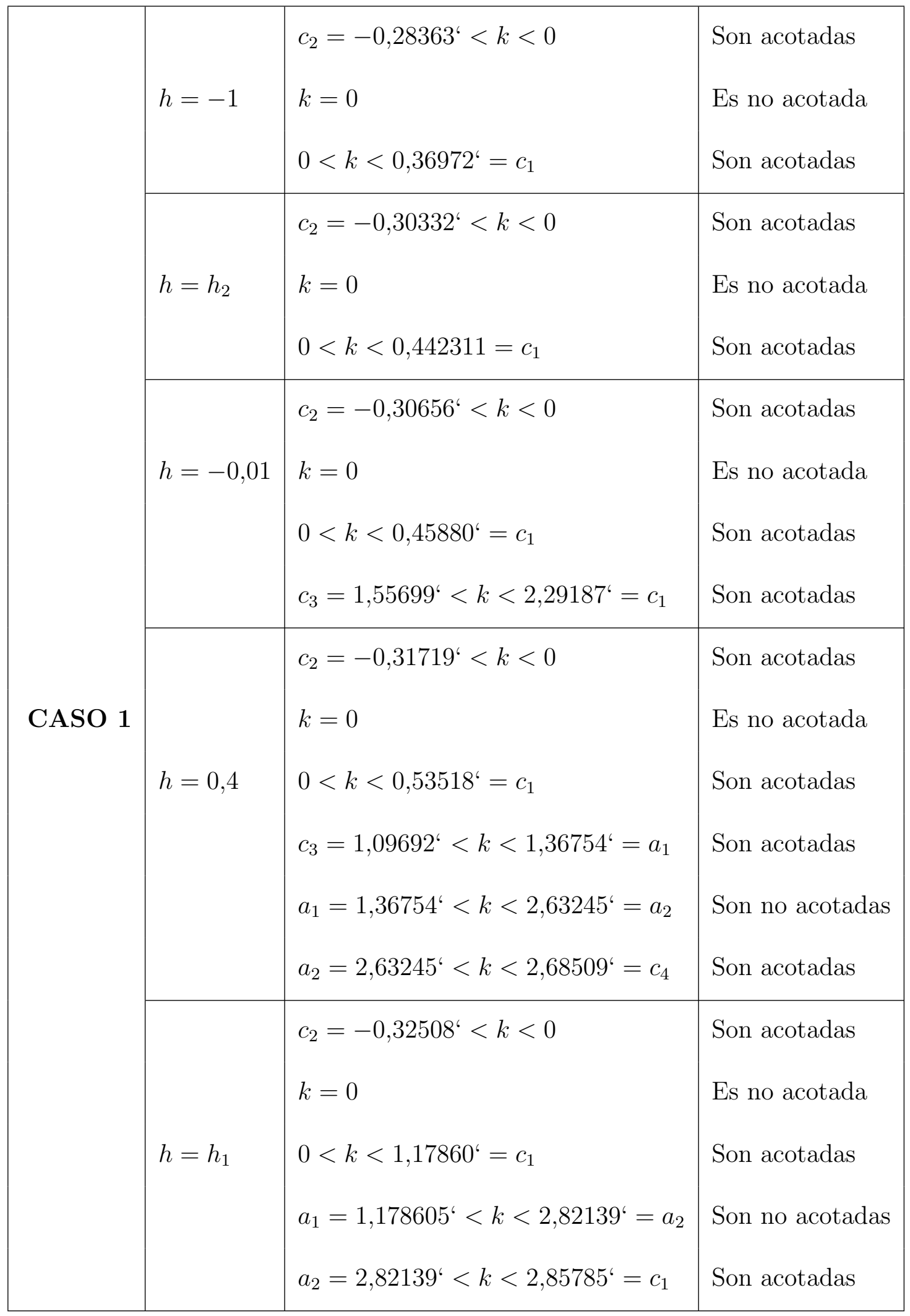

Tabla C.1: Clasificación de las curvas para el caso en que hay dos puntos críticos. 


\begin{tabular}{|l|l|l|l|}
\hline & & $c_{2}=-0,33509^{`}<k<0$ & Son acotadas \\
& & & \\
CASO 1 & $h=1$ & $0<k<1=a_{1}$ & Es no acotada \\
& & $a_{1}=1<k<3=a_{2}$ \\
& $a_{2}=3<k<3,02692^{\circ}=c_{1}$ & Son acotadas \\
Son no acotadas \\
\end{tabular}

Tabla C.2: Clasificación de las curvas para el caso en que hay dos puntos críticos.

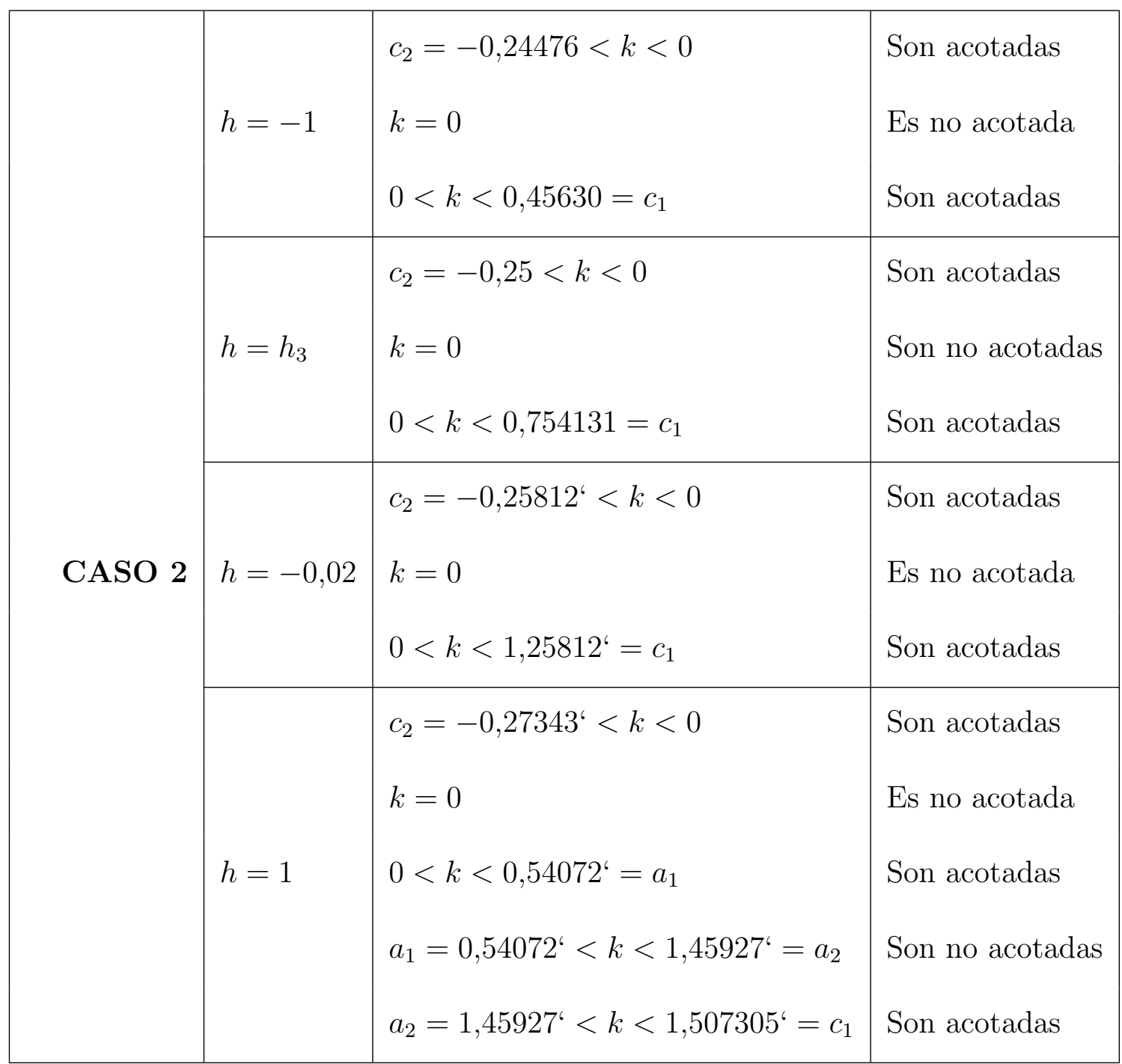

Tabla C.3: Clasificación de las curvas para el caso en que hay un punto crítico doble. 


\begin{tabular}{|c|c|c|c|}
\hline & $h=-0,129332$ & $\begin{array}{l}c_{2}=-0,256299<k<0 \\
k=0 \\
0<k<1,21121=c_{1}\end{array}$ & $\begin{array}{l}\text { Son acotadas } \\
\text { Es no acotada } \\
\text { Son acotadas }\end{array}$ \\
\hline CASO 3 & $h=1$ & $\begin{array}{l}c_{2}=-0,57878^{`}<k<0 \\
k=0 \\
a_{1}=0<k<2=a_{2} \\
a_{2}=2<k<2,05741^{`}=c_{1}\end{array}$ & $\begin{array}{l}\text { Son acotadas } \\
\text { Es no acotada } \\
\text { Son no acotadas } \\
\text { Son acotadas }\end{array}$ \\
\hline
\end{tabular}

Tabla C.4: Clasificación de las curvas para el caso en que no hay ningún punto crítico. 


\section{Apéndice D}

\section{Estudio numérico de las órbitas}

En esta sección vamos a realizar el estudio numérico de las órbitas y a dibujarlas, para el caso en que $27 \alpha \beta^{4}-128>0$, situación donde tenemos dos puntos críticos y $h=0,674 \ldots$, es decir, coincide con el valor en el punto de equilibrio. Dichas órbitas corresponden a la figura (4.8). Para el resto de los casos, bastaría con variar los valores de $\alpha, \beta, h, k$ y usar el mismo código que vamos a describir a continuación.

Observación 76 Usaremos los valores obtenidos en el apéndice anterior.

Antes de empezar definiremos los valores de $\alpha$ y $\beta$, así como el hamiltoniano en general.

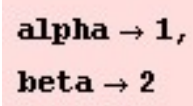

Hay que tener en cuenta que las ics son las coordenadas iniciales que introducimos y que son las que debemos ir variando según los diferentes valores de $h$ y $k$ para ver los diferentes tipos de órbitas que hay.

Empecemos ahora con cada uno de los casos:

1. Caso 1. Corresponde a la figura (4.18).

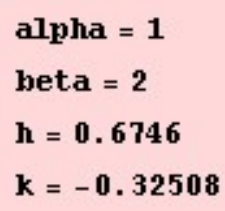




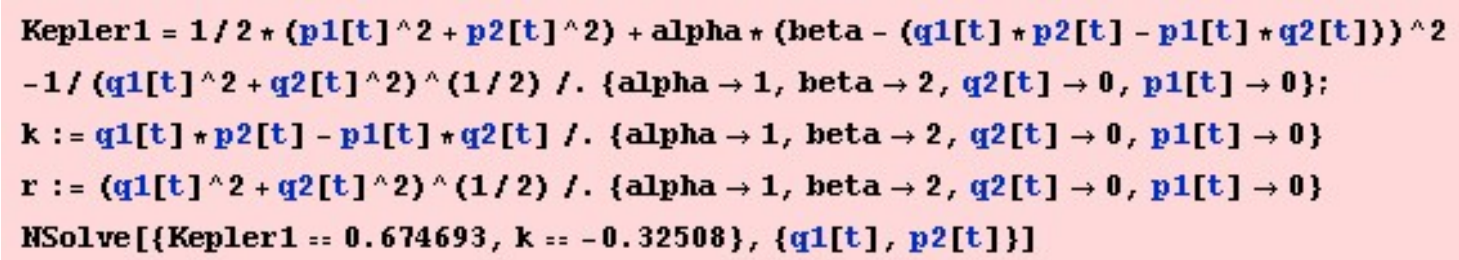

$q 1[t] \rightarrow-0.1052075346995781, p 2[t] \rightarrow 3.0898927622272643$

Una vez obtenidas las condiciones iniciales para los valores de $h$ y $k$ propios de este caso, integramos el problema de Kepler.

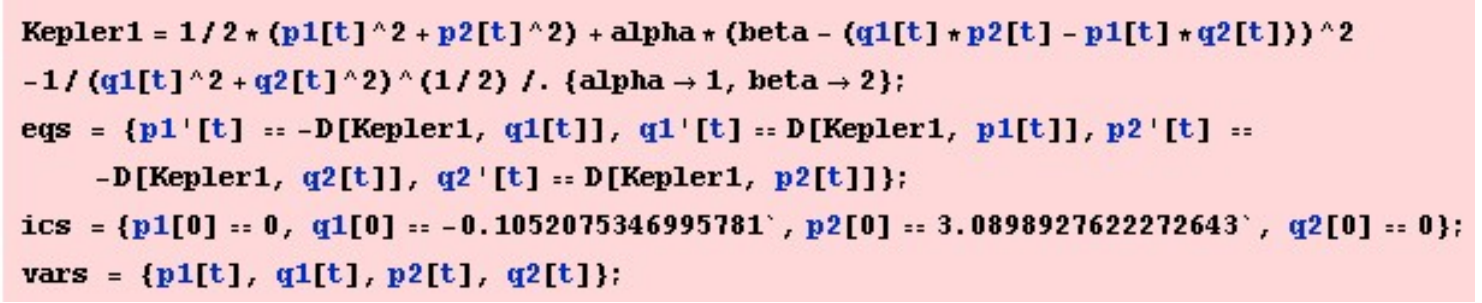

Dibujamos ahora la órbita

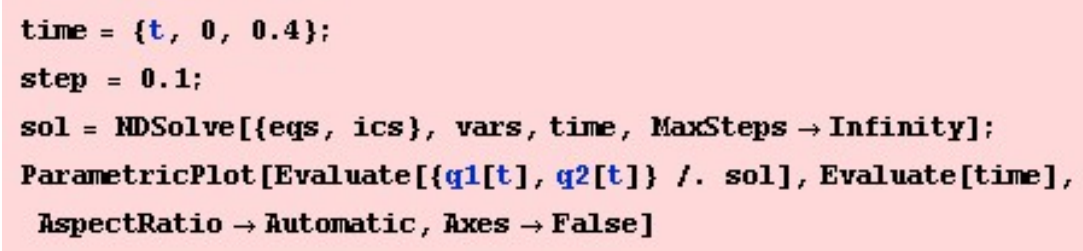

Obteniendo así la siguiente figura

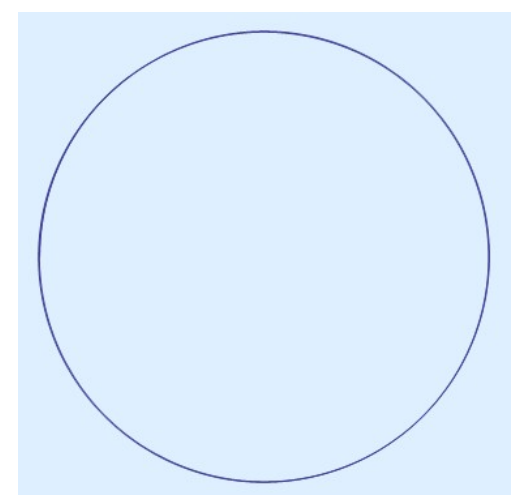

Caso de una órbita circular. 
2. Caso 2. Corresponde a la figura (4.19)

$$
\begin{aligned}
& \text { alpha }=1 \\
& \text { beta }=2 \\
& h=0.6746 \\
& \text { k }=1
\end{aligned}
$$

Kepler1 $=1 / 2 *\left(p 1[t]^{\wedge} 2+p 2[t]^{\wedge} 2\right)+\operatorname{alpha} *(\operatorname{beta}-(q 1[t] * p 2[t]-p 1[t] * q 2[t]))^{\wedge} 2$

$-1 /\left(\mathrm{q} 1[\mathrm{t}]^{\wedge} 2+\mathrm{q} 2[\mathrm{t}]^{\wedge} 2\right)^{\wedge}(1 / 2) /$. \{alpha $\rightarrow 1$, beta $\left.\rightarrow 2, \mathrm{q} 2[\mathrm{t}] \rightarrow 0, \mathrm{p} 1[\mathrm{t}] \rightarrow 0\right\}$;

$k:=q 1[t] \star p 2[t]-p 1[t] * q 2[t] / .\{a \rightarrow 1, q 2[t] \rightarrow 0, p 1[t] \rightarrow 0\}$

$r:=\left(\mathrm{q} 1[\mathrm{t}]^{\wedge} 2+\mathrm{q} 2[\mathrm{t}]^{\wedge} 2\right)^{\wedge}(1 / 2) / .\{\mathrm{a} \rightarrow 1, \mathrm{q} 2[\mathrm{t}] \rightarrow 0, \mathrm{p} 1[\mathrm{t}] \rightarrow 0\}$

HSolve [ Kepler $1==0.674693, k==1\},\{q 1[t], p 2[t]\}]$

q1 $[t] \rightarrow 2.4455188880229466^{\circ}, \mathrm{p} 2[\mathrm{t}] \rightarrow 0.4089111741878383^{\circ}$

Una vez obtenidas las condiciones iniciales para los valores de $h$ y $k$ propios de este caso, integramos el problema de Kepler.

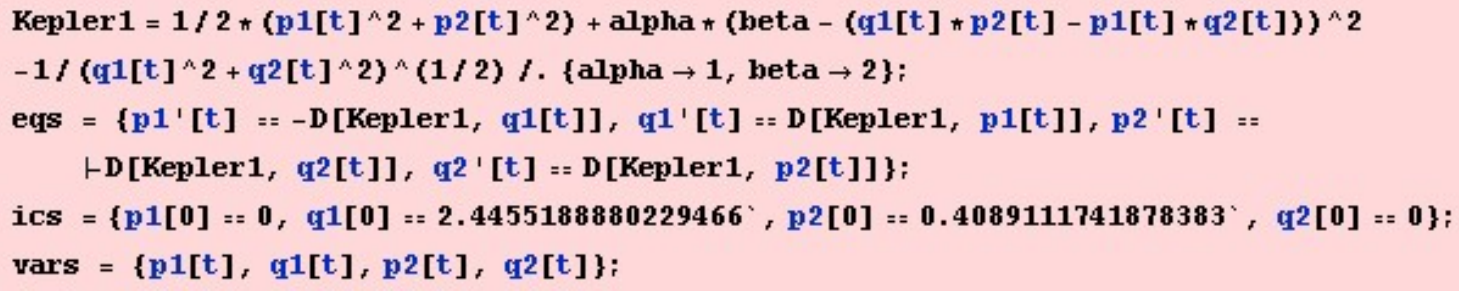

Dibujamos ahora la órbita

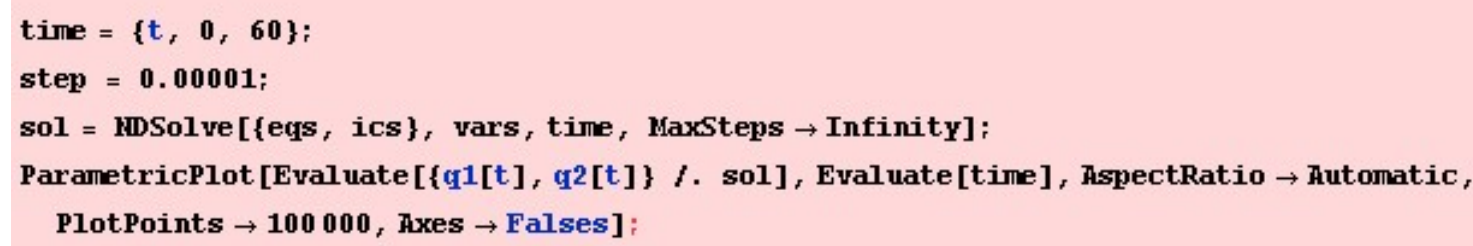


Obteniendo así la siguiente figura

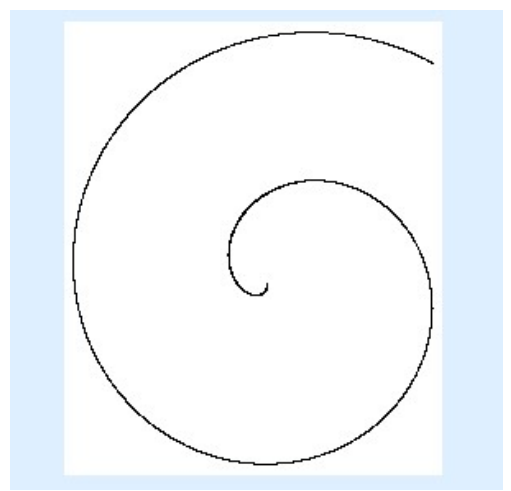

Caso de una órbita de escape.

4. Caso 4. Corresponde a la figura (4.21)

$$
\begin{aligned}
& \text { alpha }=1 \\
& \text { beta }=2 \\
& h=0.6746 \\
& k=0
\end{aligned}
$$

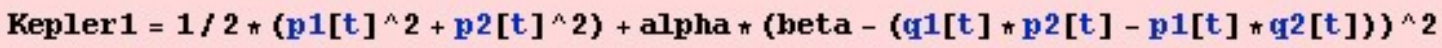

$-1 /\left(q 1[t]^{\wedge} 2+q 2[t]^{\wedge} 2\right)^{\wedge}(1 / 2) /$. \{alpha $\rightarrow 1$, beta $\left.\rightarrow 2, q 2[t] \rightarrow 0, p 1[t] \rightarrow 0\right\}$;

$k:=q 1[\mathrm{t}] * \mathrm{p} 2[\mathrm{t}]-\mathrm{p} 1[\mathrm{t}] * \mathrm{q} 2[\mathrm{t}] / .\{\mathrm{a} \rightarrow 1, \mathrm{q} 2[\mathrm{t}] \rightarrow 0, \mathrm{p} 1[\mathrm{t}] \rightarrow 0\}$

$\mathrm{r}:=\left(\mathrm{q} 1[\mathrm{t}]^{\wedge} 2+\mathrm{q} 2[\mathrm{t}]^{\wedge} 2\right)^{\wedge}(1 / 2) / .\{\mathrm{a} \rightarrow 1, \mathrm{q} 2[\mathrm{t}] \rightarrow 0, \mathrm{p} 1[\mathrm{t}] \rightarrow 0\}$

HSolve $[\{$ Kepler1 $==0.674693, k==0\},\{q 1[t], p 2[t]\}]$

Una vez obtenidas las condiciones iniciales para los valores de $h$ y $k$ propios de este caso, integramos el problema de Kepler. Dibujamos ahora la órbita

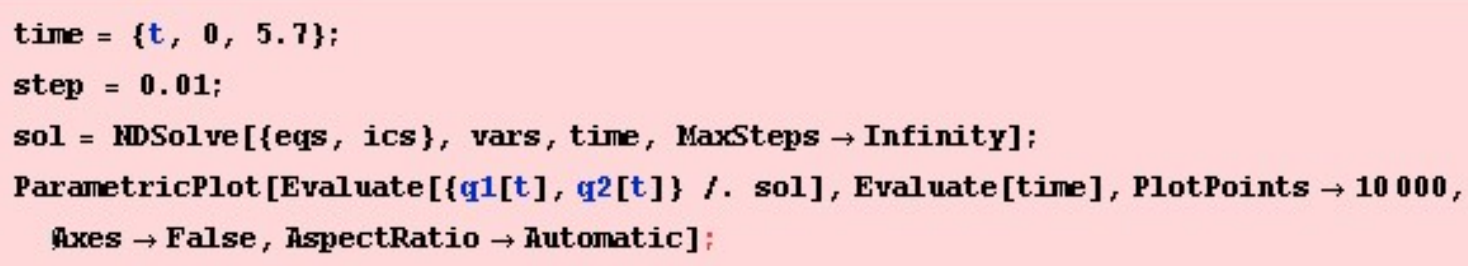


Obteniendo así la siguiente figura

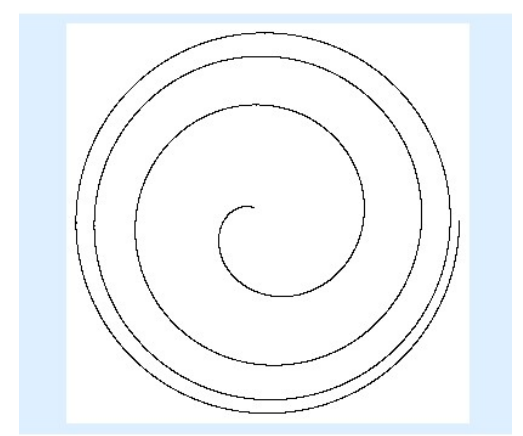

Caso de una órbita de colisión.

5. Caso 5. Corresponde a la figura (4.20)

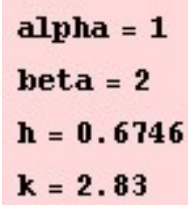

Kepler1 = $1 / 2$ * $\left(\mathrm{p} 1[\mathrm{t}]^{\wedge} 2+\mathrm{p} 2[\mathrm{t}]^{\wedge} 2\right)+\operatorname{alpha} *(\text { beta }-(\mathrm{q} 1[\mathrm{t}] * \mathrm{p} 2[\mathrm{t}]-\mathrm{p} 1[\mathrm{t}] \text { * } \mathrm{q} 2[\mathrm{t}]))^{\wedge} 2$ $-1 /\left(\mathrm{q} 1[\mathrm{t}]^{\wedge} 2+\mathrm{q} 2[\mathrm{t}]^{\wedge} 2\right)^{\wedge}(1 / 2) /$. \{alpha $\rightarrow 1$, beta $\left.\rightarrow 2, \mathrm{q} 2[\mathrm{t}] \rightarrow 0, \mathrm{p} 1[\mathrm{t}] \rightarrow 0\right\}$;

$k:=q 1[t] * \mathrm{p} 2[\mathrm{t}]-\mathrm{p} 1[\mathrm{t}] * \mathrm{q} 2[\mathrm{t}] / .\{\mathrm{a} \rightarrow \mathrm{1}, \mathrm{q} 2[\mathrm{t}] \rightarrow 0, \mathrm{p} 1[\mathrm{t}] \rightarrow 0\}$

$r:=\left(\mathrm{q} 1[\mathrm{t}]^{\wedge} 2+\mathrm{q}^{2}[\mathrm{t}]^{\wedge} 2\right)^{\wedge}(1 / 2) / .\{\mathrm{a} \rightarrow 1, \mathrm{q} 2[\mathrm{t}] \rightarrow 0, \mathrm{p} 1[\mathrm{t}] \rightarrow 0\}$

HSolve $[\{\operatorname{Kepler} 1==0.674693, k=2.83\},\{q 1[t], p 2[t]\}]$

Una vez obtenidas las condiciones iniciales para los valores de $h$ y $k$ propios de este caso, integramos el problema de Kepler.

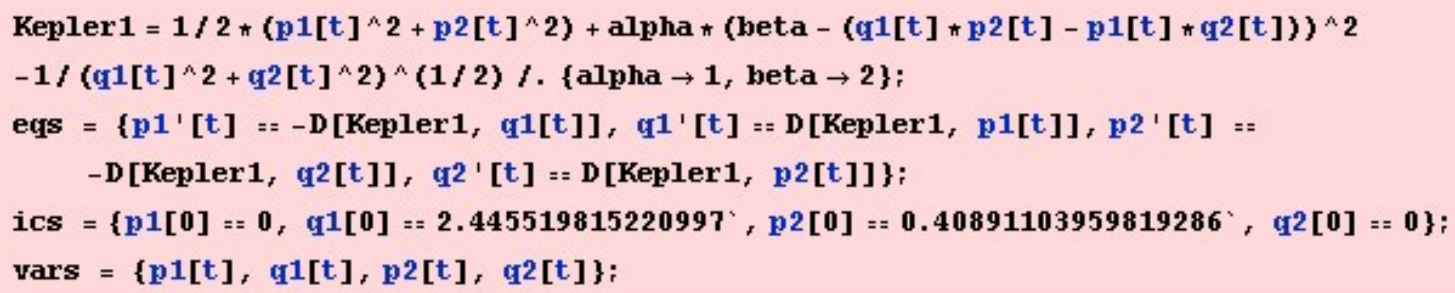


Dibujamos ahora la órbita

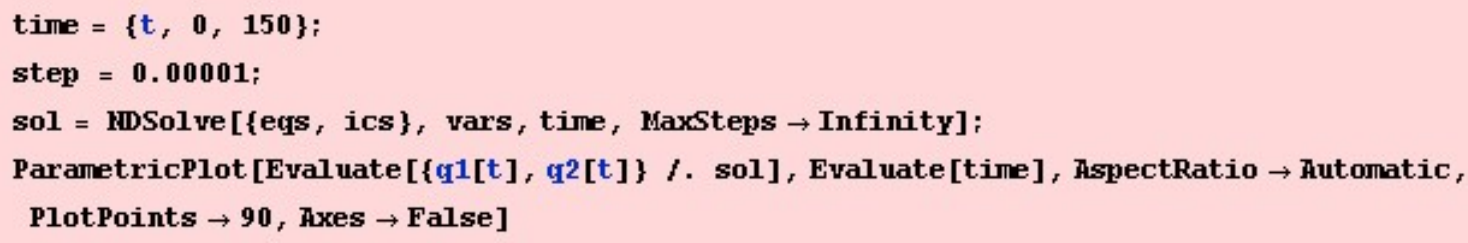

Obteniendo así la siguiente figura

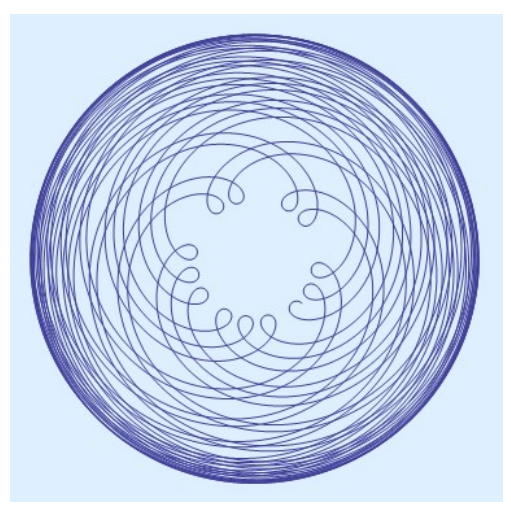

Que corresponde al caso de una órbita de cuasiperiódica. 


\section{Índice de figuras}

3.1. Giróstato con tres rotores . . . . . . . . . . . . . . . . 31

4.1. Potencial ampliado para $27 \alpha \beta^{4}-128>0$, donde $h_{1}, h_{2}$ son los puntos

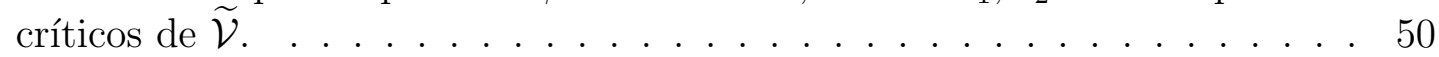

4.2. Potencial ampliado para $27 \alpha \beta^{4}-128=0$, donde $h_{3}$ es el punto crítico de $\tilde{\mathcal{V}}$. 50

4.3. Potencial ampliado para $27 \alpha \beta^{4}-128<0 \ldots \ldots \ldots \ldots \ldots$

4.4. $\mathcal{H}^{-1}(h) / S^{1}$ para $27 \alpha \beta^{4}-128>0$ y $h<h_{2}$, donde $k=p_{\theta}$ y $Q_{1}, Q_{2}$ los

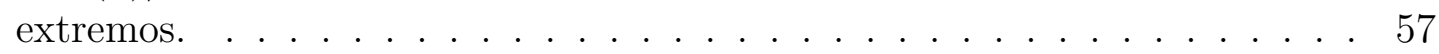

4.5. $\mathcal{H}^{-1}(h) / S^{1}$ para $27 \alpha \beta^{4}-128>0$ y $h=h_{2}$, donde $k=p_{\theta}, h_{2}$ el punto de

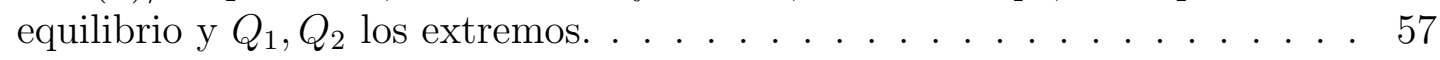

4.6. $\mathcal{H}^{-1}(h) / S^{1}$ para $27 \alpha \beta^{4}-128>0$ y $h_{2}<h<0$, donde $k=p_{\theta} \mathrm{y}$

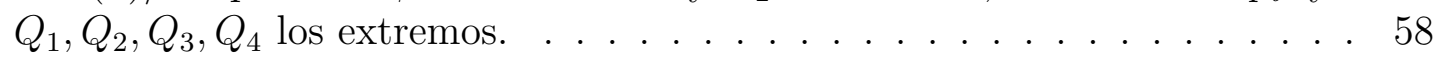

4.7. $\mathcal{H}^{-1}(h) / S^{1}$ para $27 \alpha \beta^{4}-128>0$ y $0<h<h_{1}$, donde $k=p_{\theta} \mathrm{y}$

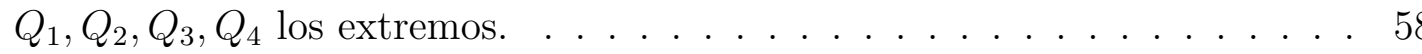

4.8. $\mathcal{H}^{-1}(h) / S^{1}$ para $27 \alpha \beta^{4}-128>0$ y $h=h_{1}$, donde $k=p_{\theta}, h_{1}$ el punto de

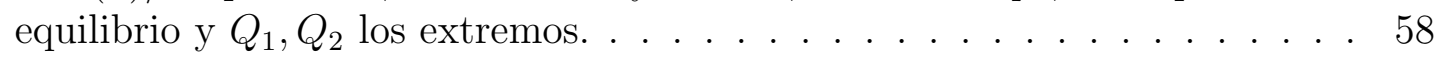

4.9. $\mathcal{H}^{-1}(h) / S^{1}$ para $27 \alpha \beta^{4}-128>0$ y $h>h_{1}$, donde $k=p_{\theta}$ y $Q_{1}, Q_{2}$ los

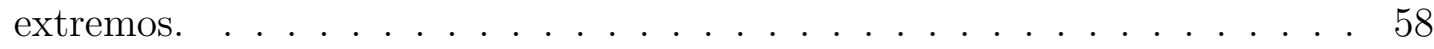

4.10. $\mathcal{H}^{-1}(h) / S^{1}$ para $27 \alpha \beta^{4}-128=0$ y $h<h_{3}$, donde $k=p_{\theta}$ y $Q_{1}, Q_{2}$ los

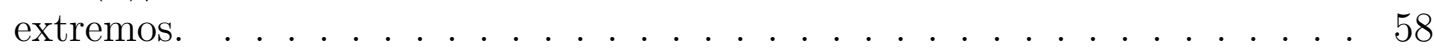

4.11. $\mathcal{H}^{-1}(h) / S^{1}$ para $27 \alpha \beta^{4}-128=0$ y $h=h_{3}$, donde $k=p_{\theta}, h_{3}$ el punto de

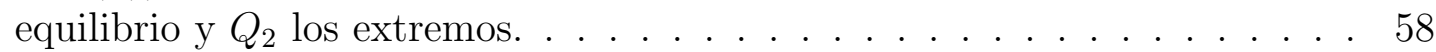

4.12. $\mathcal{H}^{-1}(h) / S^{1}$ para $27 \alpha \beta^{4}-128=0$ y $h_{3}<h<0$, donde $k=p_{\theta}$ y $Q_{1}$ y $Q_{2}$

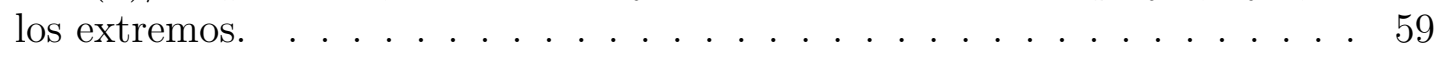

4.13. $\mathcal{H}^{-1}(h) / S^{1}$ para $27 \alpha \beta^{4}-128=0$ y $0<h$, donde $k=p_{\theta}$ y $Q_{1}$ y $Q_{2} \operatorname{los}$

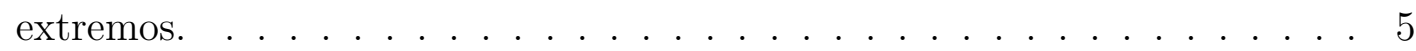

4.14. $\mathcal{H}^{-1}(h) / S^{1}$ para $27 \alpha \beta^{4}-128<0$ y $h<0$, donde $k=p_{\theta}$ y $Q_{1}, Q_{2}$ los

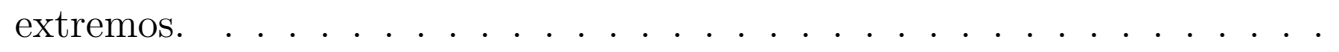

4.15. $\mathcal{H}^{-1}(h) / S^{1}$ para $27 \alpha \beta^{4}-128<0$ y $h \geq 0$, donde $k=p_{\theta}$ y $Q_{1}, Q_{2}$ los

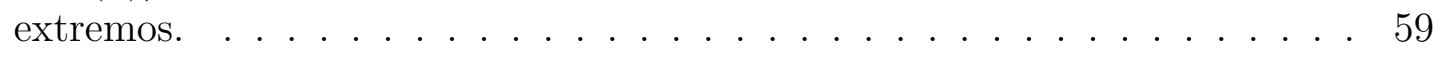

4.16. Región donde (4.28) tiene dos raíces reales distintas . . . . . . . . . 65

4.17. Región para las variables de Delaunay _. . . . . . . . . . . . . . 66

4.18. Ejemplo de una órbita circular en coordenadas cartesianas $\left(u_{1}, u_{2}\right)$ para la figura 4.8. Estas órbitas aparecen en los extremos de la superficie $m_{i}, i=$

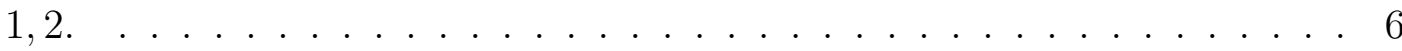

4.19. Ejemplo de una órbita periódica en coordenadas cartesianas $\left(u_{1}, u_{2}\right)$ para la figura 4.8 . 
4.20. Ejemplo de una órbita cuasiperiódica en coordenadas cartesianas $\left(u_{1}, u_{2}\right)$ para la figura 4.8 .

4.21. Ejemplo de una órbita de colisión en coordenadas cartesianas $\left(u_{1}, u_{2}\right)$ para la figura 4.8. Esta órbita ocurre cuando $k=0 . \ldots$. . . . . . . . . . .

4.22. Ejemplo de una órbita de escape en coordenadas cartesianas $\left(u_{1}, u_{2}\right)$ para la figura 4.8. Estas aparecen entre los valores $a_{1}$ y $a_{2}$ de la superficeie. . . . 67

5.1. Potencial ampliado para $\beta>0$ y $-27 / 2048+\alpha^{2} \beta^{3}>0$. . . . . . . . . 73

5.2. Potencial ampliado para $-27 / 2048+\alpha^{2} \beta^{3}=0$. . . . . . . . . . . . . 73

5.3. Potencial ampliado para $\beta>0$ y $-27 / 2048+\alpha^{2} \beta^{3}<0$. . . . . . . . . 73

5.4. Potencial ampliado para $\beta=0 . \ldots \ldots \ldots \ldots$. . . . . . . . . . . . . . . . .

5.5. Potencial ampliado para $\beta<0 . \ldots \ldots \ldots . \ldots . \ldots . \ldots 74$

5.6. $\mathcal{H}^{-1}(h) / S^{1}$ para $\beta>0,-27 / 2048+\alpha^{2} \beta^{3}$ y $\forall h$, donde $k=p_{\theta}$, y $c_{1}$ el máximo. 81

5.7. $\mathcal{H}^{-1}(h) / S^{1}$ para $-27 / 2048+\alpha^{2} \beta^{3}=0$ y $h_{1}<h$, donde $k=p_{\theta}$, y $c_{1}$ el máximo. . . . . . . . . . . . . . . . . . . 81

5.8. $\mathcal{H}^{-1}(h) / S^{1}$ para $-27 / 2048+\alpha^{2} \beta^{3}=0$ y $h=h_{1}$, donde $k=p_{\theta}$ y $h_{1}$ es el punto de equilibrio. . . . . . . . . . . . . . . . 81

5.9. $\mathcal{H}^{-1}(h) / S^{1}$ para $-27 / 2048+\alpha^{2} \beta^{3}=0$ y $h<h_{1}$, donde $k=p_{\theta}$, y $c_{1}$ el máximo. . . . . . . . . . . . . . . . . .

5.10. $\mathcal{H}^{-1}(h) / S^{1}$ para $\beta>0,-27 / 2048+\alpha^{2} \beta^{3}<0$ y $h_{2}<h$, donde $k=p_{\theta}$ y $c_{1}$ el máximo. . . . . . . . . . . . . . . . . . . . . . . . . 82

5.11. $\mathcal{H}^{-1}(h) / S^{1}$ para $\beta>0,-27 / 2048+\alpha^{2} \beta^{3}<0$ y $h=h_{2}$, donde $k=p_{\theta}$ y $c_{1}$ el máximo. . . . . . . . . . . . . . . . . . . . . 82

5.12. $\mathcal{H}^{-1}(h) / S^{1}$ para $\beta>0,-27 / 2048+\alpha^{2} \beta^{3}<0$ y $h_{3}<h<h_{2}$, donde $k=p_{\theta}$, y $c_{1}, c_{2}, c_{3}$ los extremos. . . . . . . . . . . . . . 83

5.13. $\mathcal{H}^{-1}(h) / S^{1}$ para $\beta>0,27 / 2048+\alpha^{2} \beta^{3}<0$ y $h=h_{3}$, donde $k=p_{\theta}, h_{3}$ el punto de equilibrio y $c_{1}$ el máximo.

5.14. $\mathcal{H}^{-1}(h) / S^{1}$ para $\beta>0,-27 / 2048+\alpha^{2} \beta^{3}<0$ y $h \leq h_{3}$, donde $k=p_{\theta}$ y $c_{1}$ el máximo. . . . . . . . . . . . . . . . . . . . . . 83

5.15. $\mathcal{H}^{-1}(h) / S^{1}$ para $\beta=0$ y $h_{4}<h$, donde $k=p_{\theta}$ y $c_{1}$ el máximo. . . . . . . . . . 84

5.16. $\mathcal{H}^{-1}(h) / S^{1}$ para $\beta=0$ y $h=h_{4}$, donde $k=p_{\theta}, h_{4}$ el punto de equilibrio y $c_{1}$ el máximo. . . . . . . . . . . . . . . . . 84

5.17. $\mathcal{H}^{-1}(h) / S^{1}$ para $\beta=0$ y $h<h_{4}$, donde $k=p_{\theta}$ y $c_{1}, c_{2}, c_{3}$ los extremos. . . . . 84

5.18. $\mathcal{H}^{-1}(h) / S^{1}$ para $\beta<0$ y $h_{5}<h$, donde $k=p_{\theta}$ y $c_{1}$ el máximo. . . . . . . 85

5.19. $\mathcal{H}^{-1}(h) / S^{1}$ para $\beta<0$ y $h=h_{5}$, donde $k=p_{\theta}, h_{5}$ el punto de equilibrio y $c_{1}$ el máximo. . . . . . . . . . . . . . . . . . . . . . . 85

5.20. $\mathcal{H}^{-1}(h) / S^{1}$ para $\beta<0$ y $h<h_{5}$, donde $k=p_{\theta}$ y $c_{1}, c_{2}, c_{3}$ los extremos. $\quad . \quad 85$

5.21. Región donde las variables acción-ángle están definidas para $\beta>0$. . . . 87

5.22. Región donde las variables acción-ángle están definidas para $\beta \leq 0 \ldots$. . . 87

6.1. Gráfica del potencial ampliado, para $3125 \alpha^{4} \beta^{4}-4 \beta>0$ (caso en el que no hay puntos críticos).

6.2. Gráfica de la función potencial ampliado para $3125 \alpha^{4} \beta^{4}-4 \beta=0$ (caso en el que hay un solo punto crítico, en este tenemos una raíz doble).

6.3. Gráfica de la función potencial ampliado para $3125 \alpha^{4} \beta^{4}-4 \beta<0$ y $\beta>0$ (caso en el que tenemos dos puntos críticos). . . . . . . . . . . . . . . 94

6.4. Gráfica de la función potencial para $\beta<0$ (caso en el que un único punto crítico, en este caso tenemos una raíz simple). 
6.5. Región delimitada por las desigualdades (6.10) y con la condición $3125 \alpha^{4} \beta^{4}-$ $4 \beta>0$ (caso en el que no hay puntos críticos). . . . . . . . . . . . .

6.6. Región delimitada por las desigualdades (6.10) y con la condición $3125 \alpha^{4} \beta^{4}-$ $4 \beta<0$ y $\beta>0$ (caso en el que tenemos dos puntos críticos). . . . . . . .

6.7. Región delimitada por las desigualdades (6.10) y con la condición $3125 \alpha^{4} \beta^{4}-$ $4 \beta<0$ y $\beta>0$ (caso en el que tenemos dos puntos críticos). . . . . . . .

6.8. $g^{-1}(h)=E_{h} / S^{1}, \forall h$, cuando $3125 \alpha^{4} \beta^{4}-4 \beta>0$, es decir, cuando no hay familias de puntos de equilibrio. Donde $c_{1}$ es un extremo de la superficie de energía y $k=p_{\theta} \ldots \ldots \ldots \ldots \ldots \ldots$

6.9. $g^{-1}(h)=E_{h} / S^{1}, h<h_{1}$, cuando $3125 \alpha^{4} \beta^{4}-4 \beta=0$, es decir, cuando hay una sola familia de puntos de equilibrio, en este tenemos una raíz doble. Donde $c_{1}$ es un extremo de la superficie de energía y $k=p_{\theta}$. . . . . . . 100

6.10. $g^{-1}(h)=E_{h} / S^{1}, h=h_{1}$, cuando $3125 \alpha^{4} \beta^{4}-4 \beta=0$, es decir, cuando hay una sola familia de puntos de equilibrio, en este caso tenemos una raíz doble. Donde $h_{1}$ es el punto de equilibrio y $k=p_{\theta} \ldots$. . . . . . . . . . . 100

6.11. $g^{-1}(h)=E_{h} / S^{1}, h>h_{1}$, cuando $3125 \alpha^{4} \beta^{4}-4 \beta=0$, es decir, cuando hay una sola familia de puntos de equilibrio, en este tenemos una raíz doble. Donde $c_{1}$ es un extremo de la superficie de energía y $k=p_{\theta}$. . . . . . . 100

6.12. $g^{-1}(h)=E_{h} / S^{1}, h<h_{2}$, cuando $3125 \alpha^{4} \beta^{4}-4 \beta<0$, es decir, cuando hay cuando hay dos familias de puntos de equilibrio. Donde $c_{1}$ es un extremo de la superficie de energía y $k=p_{\theta} \ldots \ldots$. . . . . . . . . . . . . . 101

6.13. $g^{-1}(h)=E_{h} / S^{1}, h=h_{2}$, cuando $3125 \alpha^{4} \beta^{4}-4 \beta<0$, es decir, cuando hay cuando hay dos familias de puntos de equilibrio. Donde $c_{1}$ es un extremo de la superficie de energía, $h_{2}$ es un punto de equilibrio y $k=p_{\theta} \ldots \ldots$. . . 101

6.14. $g^{-1}(h)=E_{h} / S^{1}, h_{2}<h<h_{3}$, cuando $3125 \alpha^{4} \beta^{4}-4 \beta<0$, es decir, cuando hay cuando hay dos familias de puntos de equilibrio. Donde $c_{1}, c_{2}$ y $c_{3}$ son tres extremos de la superficie de energía y $k=p_{\theta}$. . . . . . . . . . . . 101

6.15. $g^{-1}(h)=E_{h} / S^{1}, h=h_{3}$, cuando $3125 \alpha^{4} \beta^{4}-4 \beta<0$, es decir, cuando hay cuando hay dos familias de puntos de equilibrio. Donde $c_{1}$ es un extremos de la superficie de energía, $h_{3}$ es un punto de equilibrio y $k=p_{\theta} \ldots \ldots 101$

6.16. $g^{-1}(h)=E_{h} / S^{1}, h>h_{3}$, cuando $3125 \alpha^{4} \beta^{4}-4 \beta<0$, es decir, cuando hay cuando hay dos familias de puntos de equilibrio. Donde $c_{1}$ es un extremo de la superficie de energía y $k=p_{\theta} \ldots \ldots$. . . . . . . . . . . 102

6.17. $g^{-1}(h)=E_{h} / S^{1}, h<h_{4}$, cuando $\beta<0$, es decir, cuando hay una única familia de puntos de equilibio, en este caso tenemos una raíz simple. Donde $c_{1}$ es un extremo de la superficie de energía y $k=p_{\theta}$. . . . . . . . . . 102

6.18. $g^{-1}(h)=E_{h} / S^{1}, h=h_{4}$, cuando $\beta<0$, es decir, cuando hay una única familia de puntos de equilibio, en este caso tenemos una raíz simple. Donde $c_{1}$ es un extremo de la superficie de energía, $h_{4}$ es un punto de equilibrio y

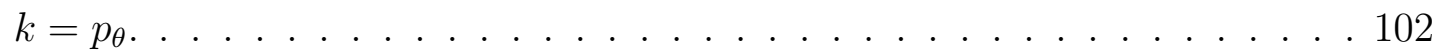

6.19. $g^{-1}(h)=E_{h} / S^{1}, h>h_{4}$, cuando $\beta<0$, es decir, cuando hay una única familia de puntos de equilibio, en este caso tenemos una raíz simple. Donde $k=p_{\theta}$. 


\section{Índice de tablas}

2.1. Ejemplos de grupos y álgebras de Lie. . . . . . . . . . . . . . . . . . . . . . 19

4.1. Clasificación topológica de las regiones de Hill para $27 \alpha \beta^{4}-128>0$. . . . 51

4.2. Clasificación topológica de las regiones de Hill para $27 \alpha \beta^{4}-128=0$. . . . 52

4.3. Clasificación topológica de las regiones de Hill para $27 \alpha \beta^{4}-128<0$. . . . 52

4.4. Extremos relativos de la superficie de energía $g^{-1}$ para $27 \alpha \beta^{4}-128>0$. . 55

4.5. Extremos relativos de la superficie de energía $g^{-1}$ para $27 \alpha \beta^{4}-128=0$. . 56

4.6. Extremos relativos de la superficie de energía $g^{-1}$ para $27 \alpha \beta^{4}-128<0$. . 56

4.7. Clasificación topológica de $E_{h}$ y $I_{h k}$ cuando $27 \alpha \beta^{4}-128>0$. . . . . . . . 60

4.8. Clasificación topológica de $E_{h}$ y $I_{h k}$ cuando $27 \alpha \beta^{4}-128=0$. . . . . . . . 61

4.9. Clasificación topológica de $E_{h}$ e $I_{h k}$ cuando $27 \alpha \beta^{4}-128<0 \ldots$. . . . . . . 62

5.1. Clasificación de valores críticos (v. c.) según los diferentes valores de $2048 \alpha^{2} \beta^{3}-$

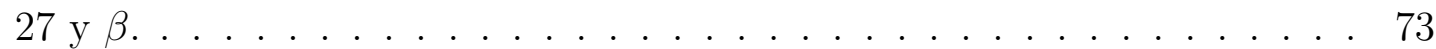

5.2. Clasificación topológica de las regiones de Hill cuando $\beta>0$ y $-27+$ $2048 \alpha^{2} \beta^{3}>0$ (véase 5.1). . . . . . . . . . . . . . . . . . . 74

5.3. Clasificación topológica de las regiones de Hill cuando $-27 / 2048+\alpha^{2} \beta^{3}=0$ (véase 5.2). . . . . . . . . . . . . . . . . . . . . . . . . . . . . . . 74

5.4. Clasificación topológica de las regiones de Hill cuando $\beta>0$ y $-27+$ $2048 \alpha^{2} \beta^{3}<0$ (véase 5.3). . . . . . . . . . . . . . . . . 75

5.5. Clasificación topológica de las regiones de Hill cuando $\beta=0$ (véase 5.4). $\quad$. 75

5.6. Clasificación topológica de las regiones de Hill cuando $\beta<0$ (véase 5.5). $\quad$. 75

5.7. Clasificación topológica de $E_{h}$ e $I_{h k}$ cuando $\beta>0$ y $-27+2048 \alpha^{2} \beta^{3}>0 . \quad 77$

5.8. Clasificación topológica de $E_{h}$ e $I_{h k}$ cuando $-27+2048 \alpha^{2} \beta^{3}=0$. . . . . . 77

5.9. Clasificación topológica de $E_{h}$ e $I_{h k}$ cuando $\beta>0$ y $-27+2048 \alpha^{2} \beta^{3}<0$. . 78

5.10. Clasificación topológica de $E_{h}$ e $I_{h k}$ cuando $\beta=0$. . . . . . . . . . . . . 79

5.11. Clasificación topológica de $E_{h}$ e $I_{h k}$ cuando $\beta<0 \ldots \ldots$. . . . . . 80

6.1. Clasificación topológica de las regiones de Hill para $3125 \alpha^{4} \beta^{4}-4 \beta>0$ (caso en el que no hay puntos críticos). Corresponde a la figura (6.1). . . . 94

6.2. Clasificación topológica de las regiones de Hill para $3125 \alpha^{4} \beta^{4}-4 \beta=0$ (caso en el que hay un solo punto crítico, en este tenemos una raíz doble). Corresponde a la figura (6.2).

6.3. Clasificación topológica de las regiones de Hill para $3125 \alpha^{4} \beta^{4}-4 \beta<0$ y $\beta>0$ (caso en el que tenemos dos puntos críticos). Corresponde a la figura

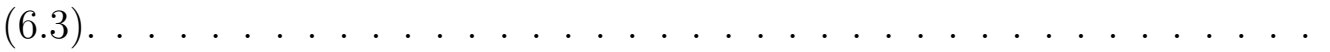

6.4. Clasificación topológica de las regiones de Hill para $\beta<0$ (caso en el que un único punto crítico, en este caso tenemos una raíz simple). Corresponde

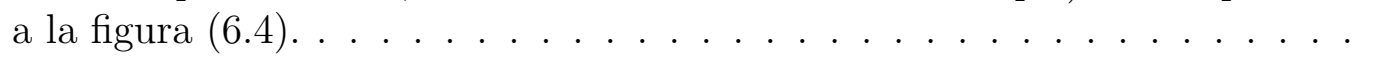


6.5. Clasificación topológica de $E_{h}$ e $I_{h k}$ cuando $3125 \alpha^{4} \beta^{4}-4 \beta>0$, es decir, cuando no hay familias de puntos de equilibrio. . . . . . . . . . . . 103

6.6. Clasificación topológica de $E_{h}$ e $I_{h k}$ cuando $3125 \alpha^{4} \beta^{4}-4 \beta=0$, es decir, cuando hay una sola familia de puntos de equilibrio. En este caso tenemos una raíz doble. . . . . . . . . . . . . . . . . . . . . . . 103

6.7. Clasificación topológica de $E_{h}$ e $I_{h k}$ cuando $3125 \alpha^{4} \beta^{4}-4 \beta<0$ y $\beta>0$, es decir, cuando hay dos familias de puntos críticos. . . . . . . . . . . . . . . 104

6.8. Tabla: Clasificación topológica de $E_{h}$ e $I_{h k}$ cuando $\beta<0$, es decir, cuando tenemos una única familia de puntos de equilibrio. En este caso tenemos una raíz simple. . . . . . . . . . . . . . . . . . . . . . 105

A.1. Resumen del número de raíces del polinomio (A.1) . . . . . . . . . . . . . . 112

B.1. Número de raíces del polinomio (B.1) para el caso en que $27 \alpha \beta^{4}-128>0$. 121 B.2. Número de raíces del polinomio (B.1) para el caso en que $27 \alpha \beta^{4}-128=0.121$ B.3. Número de raíces del polinomio (B.1) para el caso en que $27 \alpha \beta^{4}-128<0.121$

C.1. Clasificación de las curvas para el caso en que hay dos puntos críticos. . . . 136 C.2. Clasificación de las curvas para el caso en que hay dos puntos críticos. . . . 137 C.3. Clasificación de las curvas para el caso en que hay un punto crítico doble. . 137

C.4. Clasificación de las curvas para el caso en que no hay ningún punto crítico. 138 


\section{Bibliografía}

[1] A. ABAD. Astrodinámica.Editorial Bubok. (2012).

[2] R. Abraham and J. E. Marsden. Foundations in Mechanics. Addison-Wesley (1978).

[3] A. Albouy. Integral manifolds of the n-body problem. Invent. Math. 144 (1993), 463-488.

[4] V. I. ARnold. The stability of the equilibrium position of a Hamiltonian system of ordinary differential equations in the general elliptic case. Soviet Math. Dokl. 2 (1961), 247-249.

[5] V. I. Arnold. Small Denominators, II: Proof of a Theorem by A.N.Kolmogorov on the Preservation of Conditionals Periodic Motion under a Small Per-turbation of the Hamiltonian. Soviet Math. Dokl. 2 (1963), 247-249.

[6] V. I. Arnold, V. V. Kozlov and A. I. Neishtadt. Dynamical Systems III (Encyclopaedia of Mathematical Sciences). Springer Verlag. Berlin (1978).

[7] M. C. Balsas, E. S. Jiménez and J. A. Vera., The Kepler Problem in Rotatin Reference Frames: Topological Study of the Phase Flow, AIP Conference Proceedings (963) (2007), 1146-1449.

[8] M. C. Balsas, E. S. Jiménez And J. A. VerA. The motion of a gyrostat in a central gravitational field: Phase portraits of an integrable case. Journal of Nonlinear Mathematical Physics. 13 (3) (2008), 53-64.

[9] M. C. Balsas, E. S. Jiménez, J. A. Vera And A. Vigueras. Qualitative analysis of the phase flow of an integrable approximation of a generalized roto-translatory problem. Central European Journal of Physics. 7 (1) (2009), 67-78.

[10] M. C. Balsas, J. L. Guirao, E. S. JimÉnez And J. A. Vera. Qualitative analysis of the phase flow of a Manev system in a rotating reference frame. International Journal of Computer Mathematics(2009).

[11] M. C. Balsas, S. Ferrer, E. S. Jiménez And J. A. Vera. Topology and Periodic Orbits of Ring-Shaped Potentials as a Generalized 4-D Isotropic Oscillator. International Journal of Bifurcation and Chaos 20 (9) (2010), 2809-2821.

[12] M. C. Balsas, E. S. Jiménez, J. A. Vera And A. Vigueras.. Topological classification of the phase flow of the 2 dimensional main problem of the artificial satellite (Enviado a publicar a la revista 'Astrophysics and Space Science', 2016). 
[13] A. I. BARKIn ET AL. A new criterion for absolute stability: Non-linear transformation technique. International Journal of Systems Science(1983).

[14] G. D. Birkhoff. Dynamical Systems. AMS, New York (1927).

[15] A. V. Bobylev. On Vlasov-Manev Equations. I: Foundations, Properties, and Nonglobal Existence, Journal of Statistical Physics. 88 (1997), 885-911.

[16] H. E. CABral. On the integral manifolds of the n-body problem. Invent. Math. 20 (1973), 59-72.

[17] H. E. Cabral And K. R. Meyer. Stability of equilibria and fixed points of conservative systems. Nonlinearity. 12 (1999), 1351-1362.

[18] J. Casasayas, J. Llibre. Qualitative analysis of the anisotropic Kepler problem. Mem. Am. Math. Soc. 52 (1984), 312.

[19] A. CEDRIC. Bifurcation of Relative Equilibria in the Main Problem of Artificial Satellite Theory for a Prolate Body. Celestial Mechanics and Dynamical Astronomy (84) 4 (2002), 369-385.

[20] S. L. Coffey ET AL. Frozen Orbits for Satellites Close to an Earth-Like Planet, Celestial Mechanics and Dynamical Astronomy 59 (1993), 37-72.

[21] R. Cushmann and L. Bates. Global aspects of classical integrable systems. Birkhauser, Basel (1997).

[22] G. N. Duboshin. The problem of three rigid bodies. Celest. Mech.\& Dyn. Astron. 33 (1984), 31-47.

[23] A. Deprit. Canonical transformations depending on a small parameter. Celestial Mechanics. 1 (1969), 12-30.

[24] Antonio Elipe, M. Arribas And A. Riaguas. Complete analysis of bifurcations in the axial gyrostat problem. Journal of Physics A: Mathematical and General. 30 (1997), 587-601.

[25] Eugene G. Fahnestock And Daniel J. Scheeres. Simulation of the full two rigid body problem using polyhedral mutual potential and potential derivatives approach. Celestial Mechanics and Dynamical Astronomy. (96) (3-4) (2006), 317-339.

[26] C. Fanny and E. Badaoui. Relative equilibrium in the three-body problem with a rigid body. Celest. Mech.\& Dyn. Astron. 69 (1997), 293-315.

[27] J. A. FERrándiz. Movimiento de dos sólidos sometidos a atracción newtoniana. Aplicaciones. Tesis Doctoral. Universidad de Zaragoza (Spain) (1979).

[28] A. T. Fomenko. On typical topological properties of integrable Hamiltonian systems. Izv. Akad. Nauk. (SSSR) Ser. Mat 52 (1988), 378-407.

[29] F. R. Gantmacher. The Theory of Matrices. Nauka. Moscow (1966).

[30] E. A. Grebenikov. The Method of Averaging in Applications. Nauka, Moscow (1986). 
[31] H. Goldstein. Mecánica clásica. Editorial Reverté. Barcelona (1988).

[32] H. Kinoshita. Theory of the rotation of the rigid Earth. Celestial Mechanics. 15 (1977), 277-326.

[33] H. Kinoshita y J. Souchay The theory of the nutation for the rigid Earth model at the second order. Celestial Mechanics and Dynamical Astronomy. 48 (1990), 187-265.

[34] E. LeIMANis. The general problem of the motion of coupled rigid bodies about a fixed point. Springer Verlag Berlin (1965).

[35] J. LliBRE. On the restricted three-body problem when the mass parameter is small. Celestial Mechanics and Dynamical Astronomy.(28) (1982), 83-105.

[36] J. Llibre, A. E. Teruel, C. Valls and A. De la Fuente. Phase portraits of the two-body problem with Manev potential. Journal of Physics A: Mathematical and General.(34) (2001), 1919-1934.

[37] A. Maciejewski. Reduction, relative equilibria and potential in the two rigid bodies problem. Celest. Mech.\& Dyn. Astron. 63 (1995), 1-28.

[38] G. Manev. La gravitation et le principe de I'egalite de I'action et de la reaction, Comptes Rendues (178) (1924), 2159-2161.

[39] G. Manev. Die Gravitation und das Prinzip con Wirkung und Gegenwirkung, Zeitschrift fur Physik (31) (1925), 786-802.

[40] G. Manev. Le principe de la moindre action et la gravitation, Comptes Rendues (190) (1930), 963-965.

[41] G. Manev. La gravitation et 1'energie au zero, Comptes Rendues (190) (1930), $1374-1377$.

[42] F. Marchis et AL.Fine Analysis of 121 Hermione, 45 Eugenia, and 90 Antiope Binary Asteroid Systems With AO Observations. American Astronomical Society. (36) (2004), 1180.

[43] A. P. Markeev. The Libration points in Celestial Mechanics and Cosmics Dynamics. Nauka. Moscow (1978).

[44] J. E. Marsden. Lectures on Mechanics. L. M. S., Lectures Note Series (174), Cambridge University Press) (1992).

[45] J. E. Marsden And T. S. Ratiu. Introduction to Mechanics and symmetry. TAM Series (17), Springer Verlag, New York (1999).

[46] K. R. Meyer and G. R. Hall. Introduction to Hamiltonian Dynamical Systems and the N-Body Problem. Applied Mathematical Sciences. 90. Springer-Verlag, New York, (1992).

[47] F. MondÉJAR And A. Vigueras. The Hamiltonian dynamics of the two gyrostats problem. Celest. Mech.\& Dyn. Astron. 73 (1999), 303-312. 
[48] F. MondéJAr, A. Vigueras And S. Ferrer. Symmetries, reduction and relative equilibria for a gyrostat in the three-body problem. Celest. Mech.\& Dyn. Astron. 81 (2001), 45-50.

[49] J. Ostro et AL. Radar Imaging of Binary Near-Earth Asteroid. Science. (314) (2006), 1276-80.

[50] Jesús F. Palacián ET AL. Searching for periodic orbits of the spatial elliptic restricted three-body problem by double averaging. Physica D: Nonlinear Phenomena. (213) (2006), 15-24.

[51] M. Pascal. Celest. Mech.\& Dyn. Astron. 36(4) (1985), 319.

[52] V. V. Rumiantsev. P. M. M. U.R.S.S., 25(1) (1961), 9.

[53] J. Scheeres et AL. Dynamical Configuration of Binary Near-Earth Asteroid. Science. (314) (2006), 1280-83.

[54] S. Smale. Topology and Mechanics, I. Invent. Math. (10) (1970), 305-331.

[55] S. Smale. Topology and Mechanics, II. The planar n-body problem Invent. Math. (11) (1970), 45-64.

[56] A. G. Sokolskir. On the stability of a Hamiltonian System with Two Degrees of Freedom in the Case of Equal Frequencies. Prikh. Mat. Mech. 38 (1974), 791-799.

[57] A. G. Sokolskir. On the stability of an autonomous Hamiltonian System with Two Degrees of Freedom at the Resonance of the First Order. Prikh. Mat. Mech. 41 (1977), 24-33.

[58] A. G. Sokolskir. On the stability of an autonomous Hamiltonian System with Two Degrees in the Case of Null Characteristic Exponents. Prikh. Mat. Mech. 45 (1981), 441-449.

[59] J. A. VERA. Reducciones, equilibrios y estabilidad en dinámica de sólidos rígidos y giróstatos. Tesis Doctoral. Universidad Politécnica de Cartagena (Spain) (2004).

[60] J. A. Vera And A. Vigueras. Reduction, Relative Equilibria and stability for a gyrostat in the n-body problem. In: M.C. López de Silanes et al. (eds.) Monografías del Seminario Matemático García de Galdeano (VIII Journées Zaragoza-Pau de Mathematiques Appliquées et de Statistiques), vol. (31) (2004), 257-271, Servicio de Publicaciones de la Universidad de Zaragoza, Zaragoza, Spain.

[61] J. A. Vera AND A. Vigueras. Libration points of a gyrostat in a generalized three body problem. Advances in the Astronautical Sciences. (123) (2005), 2775-2795.

[62] J. A. Vera And A. Vigueras. Hamiltonian dynamics of a gyrostat in the n-body problem: relative equilibria. Celestial Mechanics and Dynamical Astronomy. (94)(3) (2006), 289-315.

[63] V. V. Vidiakin. Euler solutions in the problem of translational-rotational motion of three rigid bodies. Celest. Mech.\& Dyn. Astron. 16 (1977), 509-526. 
[64] A. Vigueras. Movimiento rotatorio de giróstatos y aplicaciones. Tesis Doctoral. Universidad Zaragoza (Spain) (1983).

[65] V. Volterra. Acta Mathematica. 22 (1899), 201.

[66] L. S. Wang, P. S. Krishnaprasad and J. H. Maddocks. Hamiltonian dynamics of a rigid body in a central gravitational field. Celest. Mech.\& Dyn. Astron. 50 (1991), 349-386.

[67] L. S. Wang, K. Y. Lian And P. T. Chen. Steady motions of gyrostat satellites and their stability. IEEE Transactions on Automatic Control. (40 (10)) (1995), 17321743 .

[68] S. G. Zhuravlev And A. A. Petrutskit. Current state of the problem of translational-rotational motion of three-rigid bodies. Soviet Astron. 34 (1990), 299-304. 
